\title{
FORMES LINÉAIRES DE LOGARITHMES EFFECTIVES SUR LES VARIÉTÉS ABÉLIENNES
}

\author{
ÉRIC GAUDRON
}

\begin{abstract}
RÉSumÉ. Nous établissons de nouvelles mesures d'indépendance linéaire de logarithmes de points algébriques d'une variété abélienne définie sur $\overline{\mathbf{Q}}$, mesures qui sont entièrement explicites en les invariants liés à la variété en question (dimension, hauteur de Faltings, degré d'une polarisation). Moyennant une hypothèse supplémentaire sur les points algébriques considérés et une constante numérique moins bonne, ces résultats généralisent ceux - comparables - de David, avec, en particulier, la présence dans le théorème principal d'un groupe algébrique qui, en variant, induit de nombreuses mesures différentes. Une caractéristique importante de la preuve est la mise en œuvre, pour la première fois dans ce contexte, de la méthode des pentes de Bost et de certains résultats de géométrie d'Arakelov qui lui sont attachés.
\end{abstract}

ABSTRACT. We prove new measures of linear independence of logarithms on an abelian variety defined over $\overline{\mathbf{Q}}$, which are totally explicit in function of the invariants of the abelian variety (dimension, Faltings height, degree of a polarization). Besides, except an extra-hypothesis on the algebraic point considered and a weaker numerical constant, we improve on earlier results (in particular David's lower bound). We also introduce into the main theorem an algebraic subgroup that leads to a great variety of different lower bounds. An important feature of the proof is the implementation of the slope method of Bost and some results of Arakelov geometry naturally associated with it.

MSC 2000 : 11J86 (11J20, 14G40, 14L05)

Mots clefs : Méthode des pentes, formes linéaires de logarithmes, approximations simultanées, méthode de Baker, réduction d'Hirata-Kohno, variété abélienne, fibré vectoriel hermitien, degré d'Arakelov, modèle de MoretBailly, hauteur.

Date: 12 septembre 2006. 
Notations. Pour $\mathbf{i}=\left(i_{1}, \ldots, i_{g}\right) \in \mathbf{N}^{g}$, on note $\mathbf{i} !=i_{1} ! \cdots i_{g}$ ! et $|\mathbf{i}|=i_{1}+\cdots+i_{g}$ la longueur de $\mathbf{i}$. D'une manière générale, la lettre $\mathrm{D}$ désigne un opérateur différentiel ; si $x=\left(x_{1}, \ldots, x_{g}\right) \in \mathbf{C}^{g}$ est un $g$-uplet de nombres complexes, alors $\mathrm{D}_{x}:=x_{1} \frac{\partial}{\partial z_{1}}+\cdots+x_{g} \frac{\partial}{\partial z_{g}}$ et si $\mathbf{w}=\left(w_{1}, \ldots, w_{g}\right)$ est une base de $\mathbf{C}^{g}$ alors $\mathrm{D}_{\mathrm{w}}^{\mathbf{i}}=\mathrm{D}_{w_{1}}^{i_{1}} \cdots \mathrm{D}_{w_{g}}^{i_{g}}$. Si $x \in \mathbf{R}$, on note $\log ^{+}(x):=\log \max \{1, x\}$ et $[x]$ la partie entière de $x$.

Soit $\mathcal{E}$ un module sur un anneau (commutatif) $R$. On note $\mathbf{V}(\mathcal{E})$ (resp. $\mathbf{P}(\mathcal{E})$ ) le spectre de l'algèbre symétrique $\mathbf{S}(\mathcal{E})$ (resp. le schéma projectif $\operatorname{Proj} \mathbf{S}(\mathcal{E})$ ) sur Spec $R$ (suivant la convention de Grothendieck). Soit $\mathcal{E}^{\vee}$ le module dual $\operatorname{Hom}_{R}(\mathcal{E}, R)$. Lorsque $\mathcal{E}=R^{N+1}$ pour un certain entier naturel $N \geq 1$, on écrira $\mathbf{P}_{R}^{N}$ au lieu de $\mathbf{P}\left(R^{N+1}\right)$. Le groupe unitaire $\left\{M \in \mathrm{M}_{N}(\mathbf{C}) ; M^{-1}={ }^{t} \bar{M}\right\}$ est noté $\mathbf{U}_{N}(\mathbf{C})$.

Si $G$ est un schéma en groupes lisse sur $\operatorname{Spec} R$, on note $t_{G}$ son espace tangent à l'origine. Lorsque $R=\mathbf{C}$, on désigne par $\exp _{G}: t_{G} \rightarrow G$ l'application exponentielle. Étant donné un point $p \in G(\mathbf{C})$, un logarithme de $p$ est un élément $u$ de $t_{G}$ tel que $\exp _{G}(u)=p$. Si $S$ est un entier naturel, $\Sigma_{p}(S)$ est l'ensemble $\{0, p, \ldots, S p\}$. Si $k$ est un corps de nombres, on désigne par $\mathcal{O}_{k}$ l'anneau des entiers de $k$ et par $\bar{k}$ une clôture algébrique de $k$. Soit $A$ une variété abélienne sur $k$ (corps de nombres) et $L$ un fibré en droites ample sur $A$. On désigne par $\mathrm{h}^{0}(A, L)$ la dimension de l'espace des sections globales $\mathrm{H}^{0}(A, L)$, par $h_{F}(A)$ la hauteur de Faltings stable normalisée de $A$ et, si $p \in A(k)$, le nombre réel $\widehat{h}_{L}(p)$ est la hauteur de Néron-Tate de $p$ (relative au fibré $L$ ). En outre, si $\sigma: k \rightarrow \mathbf{C}$ est un plongement complexe de $k$, on désigne par $A_{\sigma}(\mathbf{C})$ les points complexes de la variété abélienne $A \times_{\operatorname{Spec} \sigma(k)} \operatorname{Spec} \mathbf{C}$ et par $L_{\sigma}$ le fibré en droites sur $A_{\sigma}(\mathbf{C})$ induit par $L$.

Si $\alpha$ est un élément d'un corps de nombres $k$, on note $h(\alpha)$ la hauteur de Weil logarithmique absolue de $\alpha$, définie par la formule

$$
h(\alpha):=\frac{1}{[k: \mathbf{Q}]} \sum_{v \text { place de } k} n_{v} \log \max \left\{1,|\alpha|_{v}\right\}
$$

où $|.|_{v}$ est la valeur absolue sur le complété $k_{v}$ de $k$ en la place $v$, normalisée par $|2|_{v}=2$ si $v$ est archimédienne et $|p|_{v}=p^{-1}$ si $v$ est $p$-adique et où $n_{v}=\left[k_{v}: \mathbf{Q}_{v}\right]$ vaut 1,2 ou $\left[k_{v}: \mathbf{Q}_{p}\right]$ selon que (respectivement) $v$ est réelle, complexe ou $p$-adique (voir chap. 3 de 68]). De la sorte, si $\alpha=a / b$ est un nombre rationnel avec $a, b \in \mathbf{Z}$ premiers entre eux, alors $h(\alpha)=\log \max \{|a|,|b|\}$. Enfin, la lettre $e$ (dans un contexte mathématique) désigne $\exp (1)=2,718 \ldots$, qu'il ne faut pas confondre avec le paramètre $\mathfrak{e}$ qui apparaîtra dans le texte. 


\section{INTRODUCTION}

Ce texte est une approche effective de la théorie des formes linéaires de logarithmes au moyen de méthodes et concepts issus de la géométrie d'Arakelov. Dans le cadre d'une variété abélienne définie sur $\overline{\mathbf{Q}}$, nous présentons des mesures d'indépendances linéaires de logarithmes entièrement explicites en toutes les données du problème, y compris en les invariants naturels de la variété abélienne : dimension, hauteur de Faltings et degré d'une polarisation.

Soit $k$ un corps de nombres plongé dans $\mathbf{C}$. Soit $A$ un groupe algébrique commutatif défini sur $k$. Le groupe de Lie complexe $A(\mathbf{C})$ possède une application exponentielle exp définie sur tout l'espace tangent à l'origine $t_{A}(\mathbf{C})$ et surjective à valeurs dans $A(\mathbf{C})$. Étant donné un point $k$-rationnel $p$ de $A$, il est donc possible de considérer un antécédent $u \in t_{A}(\mathbf{C})$ du point $p \in A(\mathbf{C})$ par cette exponentielle. Un tel vecteur $u$ est appelé un logarithme de $p$. Lorsque $A$ est une variété abélienne, on dit aussi logarithme abélien.

L'objet de la théorie des formes linéaires de logarithmes consiste à étudier la distance entre un tel logarithme $u$ et un sous-espace vectoriel strict $W_{0}$ de l'espace tangent $t_{A}$, défini sur $k$. Cette problématique renferme essentiellement deux questions :

$\boldsymbol{x}$ Que peut-on dire si $u$ appartient à $W_{0} \otimes \mathbf{C}$ ?

$\boldsymbol{x}$ Dans le cas contraire, si $u \notin W_{0} \otimes \mathbf{C}$, quelle minoration de la distance $\mathrm{d}\left(u, W_{0}\right)$ peut-on obtenir en fonction des données du problème ?

Ces questions sont longtemps restées ouvertes avec seulement quelques cas particuliers étudiés (groupe linéaire commutatif de petite dimension). Au milieu des années soixante, Baker a effectué une percée spectaculaire en apportant des réponses à ces questions pour un groupe linéaire commutatif [1]. De ses travaux est issue une méthode — dite méthode de Baker — qui, généralisée aux groupes algébriques commutatifs quelconques, a permis d'aborder de manière effective de nombreux problèmes de géométrie diophantienne. En particulier, les questions posées ci-dessus ont pu être abordées en toute généralité par plusieurs auteurs et elles ont conduit aux éléments de réponses suivants.

Lorsque $u$ appartient à $W_{0} \otimes \mathbf{C}$, la première question a été résolue par Wüstholz 69] qui a démontré l'existence d'un sous-groupe algébrique connexe $B$ de $A$ tel que $u \in t_{B}(\mathbf{C})$ et $t_{B} \otimes$ $\overline{\mathbf{Q}} \subseteq W_{0} \otimes \overline{\mathbf{Q}}$. Cet énoncé est un cas particulier du théorème du sous-groupe analytique 770 . Il contient un grand nombre de résultats de transcendance sur les logarithmes de points algébriques. Le résultat de Wüstholz est qualitatif, mais, en adaptant la démonstration, l'on peut apporter d'autres renseignements sur $B$, comme par exemple une majoration de son degré géométrique, degré relatif à un fibré très ample sur une compactification équivariante de $A$. Les premiers majorants du degré de $B$ ont été obtenus par Philippon \& Waldschmidt [57, 58]. À ce problème d'effectivité du théorème de Wüstholz, l'on peut ajouter une contrainte supplémentaire en demandant que $B$ soit le plus petit (pour l'inclusion) sous-groupe algébrique connexe de $A$ tel que $u \in t_{B}(\mathbf{C})$. Bien que reposant sur des techniques similaires, cette question est plus difficile à traiter mais elle se révèle pourtant cruciale pour les applications aux théorèmes d'isogénies entre variétés abéliennes, comme l'ont montrés Masser \& Wüstholz dans une série d'articles au début des années quatrevingt-dix 48, 49,50]. Il semble désormais que cette branche de la théorie des formes linéaires de logarithmes est maintenant assez riche en résultats et démonstrations variés (dont certaines, dans le cadre d'un groupe linéaire, n'utilisent que de la géométrie des nombres sans avoir recours à une démarche «transcendante ») et qu'elle est devenue de fait autonome, tournée vers des applications spécifiques.

Compte tenu de cela, nous ne nous intéresserons dans la suite qu'au second aspect du problème de la théorie des formes linéaires de logarithmes, à savoir supposer que $u \notin W_{0} \otimes \mathbf{C}$ et fournir alors une minoration de la distance $\mathrm{d}\left(u, W_{0}\right)$ de $u$ à $W_{0} \otimes \mathbf{C}$, en fonction des invariants arithméticogéométriques de la question. Un minorant est appelé mesure d'indépendance linéaire de logarithmes. Dans cette optique, rappelons qu'il n'existe qu'un nombre relativement réduit de travaux qui s'appliquent à ce problème général (en comparaison avec la richesse de la littérature disponible dans le cas d'un groupe linéaire, voir [68]). Citons, par exemple, ceux de Masser 45, 46], Lang [41], Coates \& Lang [19], Philippon \& Waldschmidt [57, 58], Hirata-Kohno [35, 36] et l'auteur [28]. Les minorations de $\mathrm{d}\left(u, W_{0}\right)$ démontrées dans ces travaux n'ont pas toutes la même précision ni exactement le même degré de généralité mais elles ont toutes en commun d'avoir une constante résiduelle qui dépend de $A$ et qui n'est jamais explicite. Il est vrai que cette constante pourrait être théoriquement 
calculée. Toutefois les difficultés techniques pour y parvenir sont alors nombreuses et semblent devenir rapidement au moins aussi compliquées que le problème initial concernant $\mathrm{d}\left(u, W_{0}\right)$. À tel point que seuls deux résultats entièrement explicites étaient connus jusqu'alors, pour des groupes algébriques qui ne sont pas réduits à leur partie linéaire. Ils concernent tous les deux le cas dit elliptique où $A$ est un produit de $n$ courbes elliptiques. Il y a d'une part la mesure de Rémond \& Urfels 62] dans le cas ultramétrique avec $n=2$ et, d'autre part, la mesure de David [20] qui, elle, concerne le cas archimédien avec $n$ quelconque $\geq 1$.

Dans ce texte, nous montrons comment les techniques qui proviennent de l'approximation diophantienne telles la méthode de Baker, le procédé de réduction d'Hirata-Kohno, le procédé de changement de variables de Chudnosvky, peuvent s'intégrer géométriquement dans la méthode des pentes de Bost et conduire ainsi à des mesures d'indépendances linéaires de logarithmes abéliens explicites et intrinsèques. Nous expliquerons en détail au $\S 5.1$ comment se déroule la preuve en décrivant les différentes techniques qui interviennent. Les mesures obtenues tiennent compte des récentes avancées dans ce domaine et elles sont d'un point de vue théorique aussi bonnes (et même, sous certains aspects, plus générales) que celles de [28] par exemple.

Illustrons cela avec un premier cas particulier suffisamment représentatif du théorème général présenté au $\S$ 3.3. Auparavant rappelons qu'un fibré vectoriel hermitien $\overline{\mathcal{E}}=\left(\mathcal{E},\left(<,>_{\sigma}\right)_{\sigma: k \hookrightarrow \mathbf{C}}\right)$ sur $\operatorname{Spec} \mathcal{O}_{k}$ est la donnée d'un $\mathcal{O}_{k}$-module projectif $\mathcal{E}$ de type fini et, pour tout plongement complexe $\sigma$ de $k$, d'un produit hermitien $<,>_{\sigma}$ sur $\mathcal{E}_{\sigma}:=\mathcal{E} \otimes_{\sigma} \mathbf{C}$, invariant par conjugaison complexe. Un tel fibré possède un degré d'Arakelov normalisé $\widehat{\operatorname{deg}}_{n} \overline{\mathcal{E}}$ qui est le nombre réel défini par

$$
\widehat{\operatorname{deg}}_{n} \overline{\mathcal{E}}:=\frac{1}{[k: \mathbf{Q}]}\left(\log \operatorname{card}\left(\mathcal{E} /\left(\mathcal{O}_{k} e_{1}+\cdots+\mathcal{O}_{k} e_{g}\right)\right)-\sum_{\sigma: k \hookrightarrow \mathbf{C}} \frac{1}{2} \log \operatorname{det}\left(\left\langle e_{i}, e_{j}>_{\sigma}\right)_{i, j}\right)\right.
$$

où $\left\{e_{1}, \ldots, e_{g}\right\} \subseteq \mathcal{E}$ est une base de $\mathcal{E} \otimes k$. La formule du produit assure que ce nombre ne dépend pas du choix de $\left\{e_{1}, \ldots, e_{g}\right\}$.

Soit $A$ une variété abélienne de dimension $g$, définie sur un corps de nombres $k$ de degré $D$ sur Q. Soit $\sigma_{0}$ un plongement complexe de $k$ et $L$ un fibré en droites ample et symétrique sur $A$. En une place complexe $\sigma$ de $k$, la forme de Riemann $\omega_{\sigma} \in \bigwedge^{1,1} t_{A_{\sigma}}(\mathbf{C})^{v}$ du fibré en droites ample complexe $L_{\sigma} \rightarrow A_{\sigma}(\mathbf{C})$ est définie positive et elle confère à l'espace tangent $t_{A_{\sigma}}(\mathbf{C})$ une structure d'espace hermitien de la manière suivante : si $\left(e_{1}, \ldots, e_{g}\right)$ est une base de $t_{A_{\sigma}}(\mathbf{C}),\left(e_{1}^{*}, \ldots, e_{g}^{*}\right)$ la base duale de $t_{A_{\sigma}}(\mathbf{C})^{\vee}$ et si $\omega_{\sigma}$ s'écrit $\frac{i}{2} \sum_{1 \leq h, \ell \leq g} a_{h, \ell} e_{h}^{*} \wedge \overline{e_{\ell}^{*}}$ alors, pour tout $\left(z_{1}, \ldots, z_{g}\right) \in \mathbf{C}^{g}$, on a

$$
\left\|\sum_{h=1}^{g} z_{h} e_{h}\right\|_{\sigma}^{2}=\sum_{1 \leq h, \ell \leq g} a_{h, \ell} z_{h} \overline{z_{\ell}} .
$$

Notons d la distance sur $t_{A_{\sigma_{0}}}(\mathbf{C})$ associée à $\|\cdot\|_{\sigma_{0}}$. Soit $K$ une extension finie sur $k$ pour laquelle $A_{K}=A \times_{k} K$ admet réduction semi-abélienne. Il existe alors un schéma en groupes (lisse) semistable $\mathcal{A} \rightarrow \operatorname{Spec} \mathcal{O}_{K}$, de fibre générique $A_{K}$. Soit $\varepsilon: \operatorname{Spec} \mathcal{O}_{K} \rightarrow \mathcal{A}$ son élément neutre. L'espace tangent à l'origine

$$
t_{\mathcal{A}}:=\varepsilon^{*}\left(\bigwedge^{g} \Omega_{\mathcal{A} / \mathcal{O}_{K}}^{1}\right)^{v}
$$

s'identifie à un $\mathcal{O}_{K}$-module projectif de type fini et, pour chaque place complexe $\sigma$ de $K$, le $\mathbf{C}$ espace vectoriel $t_{\mathcal{A}} \otimes_{\sigma} \mathbf{C} \simeq t_{\left(A_{K}\right)_{\sigma}}(\mathbf{C})$ est muni de la norme $\|\cdot\|_{\sigma}$ (en notant encore $\sigma$ la place induite sur $k$ ). En particulier chaque sous-espace vectoriel $W_{0}$ de $t_{A}$ (dont $t_{A}$ lui-même) possède une structure de fibré vectoriel hermitien $\overline{\mathcal{W}_{0}}$ sur $\operatorname{Spec} \mathcal{O}_{K}$ en munissant le $\mathcal{O}_{K}$-module saturé $\mathcal{W}_{0}:=\left(W_{0} \otimes_{k} K\right) \cap t_{\mathcal{A}}$ de la restriction de la norme $\|.\|_{\sigma}$ à $W_{0} \otimes_{\sigma} \mathbf{C}$ pour tout $\sigma: K \hookrightarrow \mathbf{C}$. Posons alors

$$
\check{h}\left(W_{0}\right):=\widehat{\operatorname{deg}}_{\mathrm{n}} \overline{t_{\mathcal{A}}}-\widehat{\operatorname{deg}}_{\mathrm{n}} \overline{\mathcal{W}_{0}} .
$$

Nous sommes maintenant en mesure d'énoncer le résultat évoqué au début de ce paragraphe.

Théorème 1.1. Avec les notations ci-dessus, soit $u \in t_{A_{\sigma_{0}}}(\mathbf{C})$ d'exponentielle $p:=\exp _{A_{\sigma_{0}}(\mathbf{C})}(u)$ $k$-rationnelle. Désignons par $\widehat{h}_{L}(p)$ la hauteur de Néron-Tate de p relative à la polarisation L. Soit 
$W_{0}$ un sous-espace vectoriel de $t_{A}$, défini sur $k$, et de codimension $t \geq 1$. Considérons $a, b, \mathfrak{e}$ des nombres réels strictement positifs vérifiant les conditions suivantes :

$$
\mathfrak{e} \geq e, \quad \log a=\max \left\{\widehat{h}_{L}(p), \frac{\left(\mathfrak{e}\|u\|_{\sigma_{0}}\right)^{2}}{D}\right\}, \quad \log b=D \max \left\{1, \check{h}\left(W_{0}\right)\right\} .
$$

Soit a l'entier défini par la formule

$$
\mathfrak{a}:=\left[\frac{D}{\log \mathfrak{e}} \max \left\{1, h_{F}(A)+\frac{1}{2} \log \mathrm{h}^{0}(A, L), \log ^{+}\left(\frac{D}{\log \mathfrak{e}}\right), \log ^{+} \log a\right\}\right]+1 .
$$

Supposons qu'aucun des multiples entiers non nuls de p n'appartienne à une sous-variété abélienne stricte de $A(\bar{k})$. Alors dans ces conditions le vecteur $u$ n'appartient pas à $W_{0} \otimes_{\sigma_{0}} \mathbf{C}$ et la distance $\mathrm{d}\left(u, W_{0}\right)$ est minorée comme suit :

$$
\log \mathrm{d}\left(u, W_{0}\right) \geq-c_{1} \mathfrak{a}^{1 / t}(\mathfrak{a} \log \mathfrak{e}+\log b)\left(1+\frac{D \mathfrak{a}}{\log \mathfrak{e}} \log a\right)^{g / t}
$$

avec $c_{1}:=(10(g+t))^{13 \frac{(g+t)^{2}}{t}}$.

Outre l'aspect entièrement effectif de (2) qui ressort clairement, cette mesure tient compte des progrès récents accomplis dans ce domaine, en particulier en ce qui concerne le paramètre log $b$. En effet, l'on constate que le membre de droite de l'inégalité (2) est linéaire en log b, et, de ce fait, optimal en ce paramètre, à l'instar des mesures présentées dans les travaux de David \& HirataKohno [23] (cas elliptique) et de l'auteur [28]. Rappelons que le problème d'une dépendance linéaire en la hauteur du sous-espace (mesurée par $\log b$ ) avait été soulevé par Lang au milieu des années soixante, dans le cadre des courbes elliptiques, en relation avec le théorème de Siegel sur la finitude du nombre de points entiers sur une courbe algébrique de genre $\geq 1$ (voir [40]). Les premières mesures générales obtenues par Philippon \& Waldschmidt comportaient un terme en $(\log b)^{(g+1) / t}$. Puis, en 1991, Hirata-Kohno obtint une mesure en $(\log b)(\log \log b)^{(g+1) / t}$ grâce à un « procédé de réduction »novateur. Nous décrirons au $\S 5.1$ la traduction géométrique de cette idée qui interviendra de manière cruciale au cours de la preuve. En couplant cette réduction avec le procédé de changement de variables de Chudnovsky qui permet de mieux contrôler les dénominateurs de nombres algébriques qui apparaissent au cours de la preuve, l'on obtient la dépendance linéaire en $\log b$.

Nous démontrerons à la fin du $\S 5.11$ la conséquence suivante du théorème 1.1, qui correspond au cas $W_{0}=\{0\}$.

Corollaire 1.2. Sous les mêmes hypothèses que le théorème 1.1, on a

$$
\log \|u\|_{\sigma_{0}} \geq-c_{2} D \max \left\{1, \widehat{h}_{L}(p), h_{F}(A)+\frac{1}{2} \log h^{0}(A, L)\right\}
$$

avec 目: $=(22 g)^{52 g}$.

Plusieurs auteurs ont obtenu par voie directe ce type de minoration [4, 37, 55]. Mais, à ma connaissance, il n'existait pas de tel résultat entièrement explicite. En outre, bien que réduit à l'essentiel, cet énoncé ne découle pas directement des définitions. Cela contraste singulièrement avec les énoncés du même type pour un groupe linéaire. Par exemple, pour le groupe affine $\mathbb{G}_{\mathrm{a}}$, dont l'exponentielle est l'identité, le logarithme se confond avec le point et nous disposons de l'inégalité de Liouville :

$$
\forall \alpha \in k \backslash\{0\}, \quad \log |\alpha|_{\sigma_{0}} \geq-D h(\alpha),
$$

immédiate à partir de la définition de la hauteur de Weil $h$. Pour le groupe multiplicatif $\mathbb{G}_{\mathrm{m}}$, nous avons de même l'inégalité, pour tout $\alpha \in k \backslash\{0\}$ tel que $\log \alpha \neq 0$ :

$$
\log |\log \alpha| \geq-D(h(\alpha)+\log 2) .
$$

Cette dernière s'obtient en appliquant l'inégalité de Liouville à $\alpha-1$ et en observant que $|\alpha-1| \leq$ $|\log \alpha| \max \{1,|\alpha|\}$ (voir également le lemme 3 de [2]). L'on peut noter qu'en remplaçant $\alpha$ par $\alpha_{1}^{b_{1}} \cdots \alpha_{n}^{b_{n}}\left(\alpha_{i} \in k \backslash\{0\}, b_{i} \in \mathbf{Z}\right)$, l'inégalité (3) devient

$$
\log \left|b_{1} \log \alpha_{1}+\cdots+b_{n} \log \alpha_{n}\right| \geq-D\left|b_{1}\right| h\left(\alpha_{1}\right)-\cdots-D\left|b_{n}\right| h\left(\alpha_{n}\right)-D \log 2
$$


Cette inégalité est en quelque sorte une inégalité primitive de la théorie des formes linéaires de logarithmes. L'espace $W_{0}$ est l'hyperplan $b_{1} z_{1}+\cdots+b_{n} z_{n}=0$ dans l'espace tangent à $\mathbb{G}_{\mathrm{m}}^{n}$ et la hauteur $\log b$ de $W_{0}$ n'est rien d'autre que $\max _{i} \log \left|b_{i}\right|$. Le membre de droite de cette inégalité est exponentiel en $\log b$. Il est donc très éloigné de la dépendance linéaire attendue. Elle s'avère néanmoins intéressante lorsque les $b_{i}$ sont petits et que l'on regarde la hauteur des $\alpha_{i}$. Dans ces conditions, elle est même nettement plus avantageuse que la minoration (beaucoup plus difficile à démontrer)

$$
\log \left|b_{1} \log \alpha_{1}+\cdots+b_{n} \log \alpha_{n}\right| \geq-c_{3}(\log b) \prod_{i=1}^{n} \max \left\{1, h\left(\alpha_{i}\right),\left|\log \alpha_{i}\right|\right\}
$$

où $c_{3}$ est une constante qui ne dépend que de $n$ et $D$, obtenue par Baker \& Wüstholz [2]. Ces observations nous amènent à poser la même question non plus pour le groupe multiplicatif $\mathbb{G}_{\mathrm{m}}^{n}$ mais pour la variété abélienne $A$. Autrement dit, que signifie généraliser l'inégalité (4) à $A$ et cela est-il possible ? Si, pour calquer la situation du cas linéaire, nous supposons provisoirement que $A$ est la variété produit $A_{1} \times \cdots \times A_{n}$, nous constatons en premier lieu que le pendant abélien d'une telle inégalité ne peut pas s'obtenir en remplaçant simplement $u$ par $b_{1} u_{1}+\cdots+b_{n} u_{n}$ dans le corollaire 1.2 (les $u_{i}$ étant des logarithmes de points $p_{i} \in A_{i}(k)$ ). Même en prenant les $b_{i}$ dans l'anneau des endomorphismes de $A$ (et non plus seulement $\mathbf{Z}$ ), nous ne pourrions obtenir ainsi que certaines formes linéaires particulières. Une manière d'éviter cet écueil est de considérer une sous-variété abélienne $B$ de $A_{\bar{k}}$ et de minorer la distance $\mathrm{d}\left(u, t_{B_{\sigma_{0}}}(\mathbf{C})\right)$ de $u$ à l'espace tangent de $B$. Dans cette direction, nous démontrerons le

Théorème 1.3. Soit $\mathfrak{e}$, a, a les paramètres définis dans le théorème 1.1. Supposons, comme dans ce théorème, qu'aucun multiple entier non nul de $p$ n'appartienne à une sous-variété abélienne stricte de A. Alors, pour toute sous-variété abélienne $B$ de $A_{\bar{k}}$, de codimension $t \geq 1$, on a

$$
\log \mathrm{d}\left(u, t_{B_{\sigma_{0}}}(\mathbf{C})\right) \geq-c_{4}\left(\mathfrak{a} \operatorname{deg}_{L} B\right)^{1 / t}\left(\mathfrak{a} \log \mathfrak{e}+\log \operatorname{deg}_{L} B\right)\left(1+\frac{D \mathfrak{a}}{\log \mathfrak{e}} \log a\right)
$$

avec $c_{4}:=(10(g+t))^{26(g+t)}$.

Cette minoration est de nature fondamentalement différente de la minoration que nous pouvons déduire du théorème 1.1 en choisissant $W_{0}=t_{B}$, car, à l'instar de l'inégalité (4), le membre de droite est exponentiel en la hauteur de $B$ (hauteur qui est du même ordre que $\log \operatorname{deg}_{L} B$, voir $\S$ 4.4.1) et, simultanément, quasi linéaire en la hauteur du point $p$. La dépendance exacte en $\widehat{h}_{L}(p) \leq \log a$ est $(\log a)(\log \log a)^{2+1 / t}$ et n'est donc pas tout à fait linéaire en $\log a$. Nous montrons dans [29] comment, avec une analyse différente et plus fine de ce cas particulier, l'on peut supprimer le $\log \log a$ qui subsiste. Quoi qu'il en soit, le théorème 1.3 apparaît comme une variante abélienne de l'inégalité (4). Par ailleurs, en choisissant $B=\{0\}$, l'on peut en déduire de nouveau le corollaire 1.2. À ce stade de la discussion, cela peut paraître surprenant, mais nous verrons que les théorèmes 1.1 et 1.3 découlent d'un seul et même théorème, énoncé au $\S 3.3$ (théorème 3.1). Il serait néanmoins intéressant d'établir directement le théorème 1.3, sans avoir recours, par exemple, à des méthodes d'approximations diophantiennes.

Remerciements : Ce texte est l'aboutissement d'un travail assez long, commencé au début de ma thèse, et dans laquelle se trouve une version plus faible des résultats présentés ici. Je tiens à remercier G. Diaz, S. David et M. Waldschmidt qui ont co-dirigé ma thèse, ainsi que J.-B. Bost et N. Hirata-Kohno qui étaient les rapporteurs. Je les remercie, ainsi que M. Laurent, pour l'intérêt qu'ils ont manifestés pour ce travail et toute leur aide. J'ai préparé ma thèse dans l'équipe de Théorie des Nombres de Saint-Étienne (France) où j'ai bénéficié de conditions de travail exceptionnelles (pour le simple doctorant que j'étais), tant sur les plans scientifique, humain que financier. Je leur en suis extrêmement redevable. Je remercie également D. Bertrand et P. Philippon avec lesquels j'ai eu de nombreuses discussions qui ont permis d'améliorer et d'éclairer certains points techniques (comme la possibilité pour les paramètres $D_{i}$ d'être nuls). Je remercie G. Rémond pour sa lecture attentive des différentes versions de ce texte. Enfin, je remercie les rapporteurs pour leurs nombreuses remarques qui m'ont permis de simplifier et de rendre plus rigoureux un nombre incalculable de détails tout en améliorant la rédaction. 


\section{Organisation du texte}

Comme nous l'avons dit, les résultats présentés dans l'introduction découlent tous du théorème 3.1 énoncé au $\S 3.3$. Comme celui-ci requiert au préalable quelques notations et hypothèses, nous les avons introduites séparément aux $\S \S 3.1$ et 3.2. Par ailleurs, afin de faciliter la lecture de la preuve de ce théorème, nous rappelons au $\S$ plusieurs points de la théorie des inégalités de pentes ainsi que d'autres résultats annexes qui interviennent dans la preuve. Ces rappels visent à donner un sens précis à certaines notions (hauteurs, pentes) relativement classiques tout en établissant quelques variantes de théorèmes déjà existants dans la littérature mais présentés sous une forme différente. Ce paragraphe 1 peut se lire de manière indépendante du reste du texte. La partie qui suit ( $\S$ 5) est consacrée à la démonstration proprement dite du théorème 3.1. Nous commençons au $\S 5.1$ par expliquer le déroulement de la démonstration et chacun des paragraphes qui suivent (jusqu'au $\S 5.11$ correspondent à une étape de celle-ci. Une fois le théorème 3.1 établi, le $\S 6$ est consacré au cas particulier d'un produit de courbes elliptiques. L'objectif est de faciliter la comparaison avec la mesure de David. Enfin, dans l'appendice, nous analysons le rôle joué par les diverses constantes qui interviennent au cours de la preuve au $\S 5$ et les conditions qu'elles doivent satisfaire. Outre la justification a posteriori des choix des constantes $\phi_{12}, \ldots, h$ introduites tout au début de la démonstration ( $\$ 5.3$, p. 30), cela permet également d'affiner la constante du théorème 3.1 dans le cas particulier elliptique étudié au $\S 6$.

TABLE DES MATIÈRES

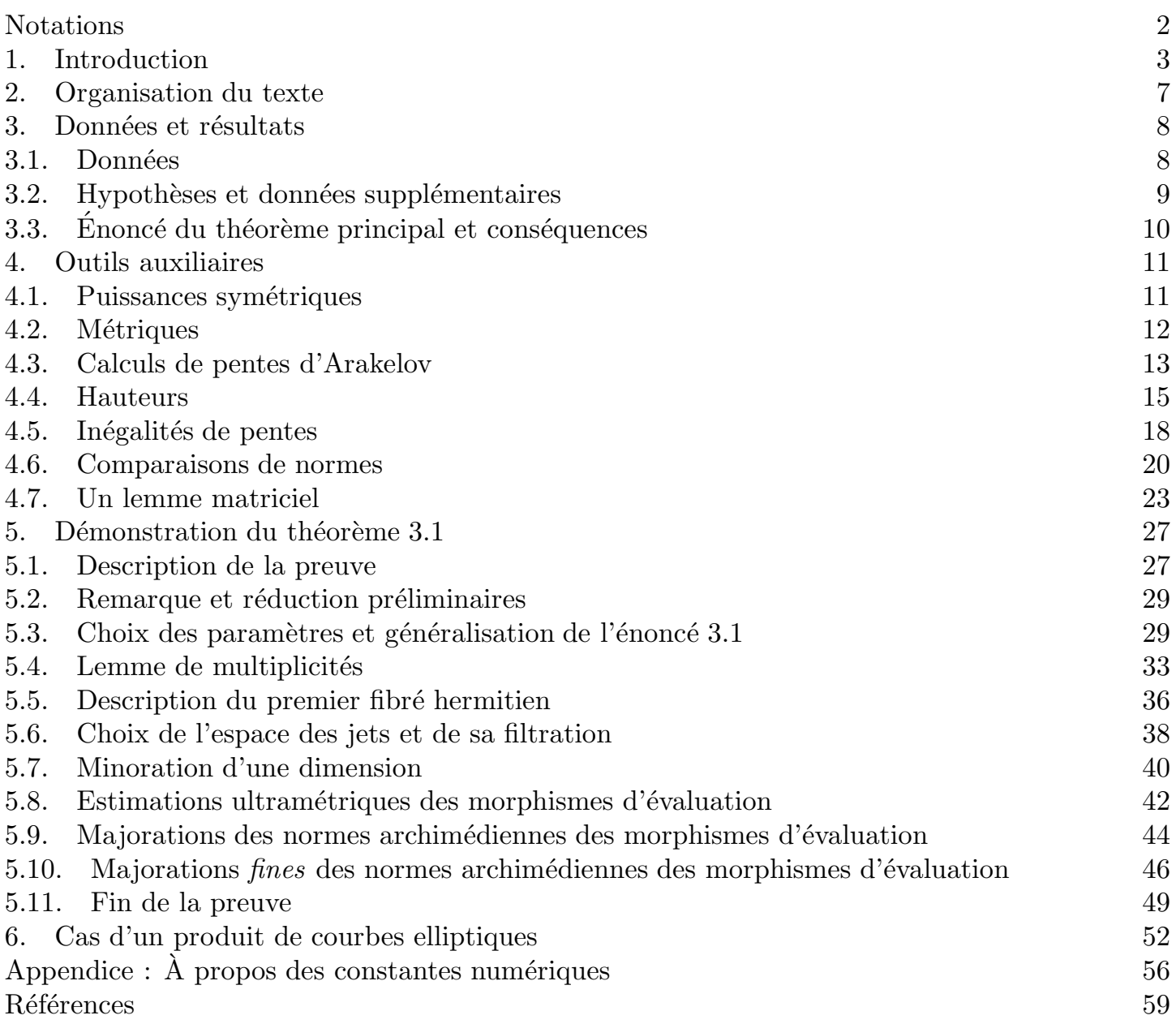




\section{Données et RÉsultats}

Nous définissons ici les données indispensables pour énoncer en toute généralité le théorème 3.1. Elles seront utilisées dans toute la suite. Les notations sont compatibles avec celles de l'introduction (qui peuvent n'en être toutefois que des cas particuliers).

3.1. Données. Soit $n$ un entier naturel $\geq 1$ et $k$ un corps de nombres de degré $D$, vu comme souscorps de $\mathbf{C}$ après avoir fixé un plongement complexe $\sigma_{0}: k \hookrightarrow \mathbf{C}$. Pour tout entier $i \in\{1, \ldots, n\}$, considérons une variété abélienne $A_{i}$, de dimension $g_{i}$, définie sur $k$ ainsi qu'un fibré en droites ample et symétrique $L_{i}$ sur $A_{i}$. Posons $A:=A_{1} \times \cdots \times A_{n}$, puis $g:=\operatorname{dim} A$ et notons $L$ le produit externe $L_{1} \otimes \cdots \otimes L_{n}$. Il est commode aussi de poser

$$
\mathrm{h}_{F}:=\max _{1 \leq i \leq n}\left\{h_{F}\left(A_{i}\right)+\frac{1}{2} \log \mathrm{h}^{0}\left(A_{i}, L_{i}\right)\right\} .
$$

Pour $i \in\{1, \ldots, n\}$, soit $u_{i}$ un élément de $t_{A_{i}, \sigma_{0}}(\mathbf{C})$ tel que

$$
p_{i}:=\exp _{A_{i, \sigma_{0}}(\mathbf{C})}\left(u_{i}\right) \in A_{i}(k) .
$$

Soit $W_{0}$ un sous- $k$-espace vectoriel de $t_{A}$ de codimension $t \geq 1$ et $u_{0}$ un élément de $t_{A}(k) / W_{0}$. Soit $G_{0}:=\mathbf{V}\left(\left(t_{A} / W_{0}\right)^{v}\right)$ le schéma en groupes affine sur Spec $k$ associé à l'espace vectoriel $t_{A} / W_{0}$. Définissons $G$ comme le groupe algébrique $G_{0} \times A$ et $p$ comme le point $\left(u_{0}, p_{1}, \ldots, p_{n}\right)$ de $G(k)$. Soit $\lambda$ la projection canonique $t_{A} \rightarrow t_{A} / W_{0}$. Considérons alors l'espace $W$ défini comme le graphe de $\lambda$ dans $t_{G}=\left(t_{A} / W_{0}\right) \oplus t_{A}$ ou, autrement dit,

$$
W:=\left\{\lambda(z) \oplus z, z \in t_{A}\right\} .
$$

Soit $X_{0}:=\mathbf{P}\left(k \oplus\left(t_{A} / W_{0}\right)^{v}\right)$ la fermeture projective de $G_{0}$ et $\iota_{0}: G_{0} \rightarrow X_{0}$ l'immersion ouverte canonique. Par ailleurs, pour tout $h \in\{1, \ldots, n\}$, le choix (arbitraire) d'une $k$-base de sections globales du fibré en droites très ample $L_{h}^{\otimes 3}$ sur $A_{h}$ détermine un plongement $\iota_{h}: A_{h} \rightarrow \mathbf{P}\left(\mathrm{H}^{0}\left(A_{h}, L_{h}^{\otimes 3}\right)\right)$. Nous notons $\iota$ le plongement produit

$$
G \hookrightarrow \mathbf{P}:=X_{0} \times \prod_{i=1}^{n} \mathbf{P}\left(\mathrm{H}^{0}\left(A_{i}, L_{i}^{\otimes 3}\right)\right)
$$

induit par les $\iota_{i}$ pour $0 \leq i \leq n$. Les degrés géométriques des sous-groupes de $G$, plongés dans $\mathbf{P}$ via $\iota$, sont relatifs au faisceau $\mathcal{O}(1, \ldots, 1)$ sur $\mathbf{P}$. Par exemple, en utilisant la formule classique de Frobenius

$$
\mathrm{h}^{0}\left(A_{i}, L_{i}\right)=\frac{\operatorname{deg}_{L_{i}} A_{i}}{g_{i} !},
$$

on a

$$
\operatorname{deg}_{\iota} G=3^{g} \frac{(g+t) !}{t !} \prod_{i=1}^{n} \mathrm{~h}^{0}\left(A_{i}, L_{i}\right) .
$$

Étant donné un plongement complexe $\sigma$ de $k$, nous reprenons la norme hermitienne $\|\cdot\|_{\sigma} \operatorname{sur} t_{A_{\sigma}}(\mathbf{C})$ définie dans l'introduction (formule (1), p. 四), au moyen de la forme de Riemann de $L_{\sigma} \rightarrow A_{\sigma}(\mathbf{C})$. Par quotient et somme orthogonale, cette norme s'étend à l'espace $t_{G_{\sigma}}(\mathbf{C})=\left(t_{A} / W_{0}\right)_{\sigma}(\mathbf{C}) \oplus$ $t_{A_{\sigma}}(\mathbf{C})$, extension que l'on note encore $\|\cdot\|_{\sigma}$. Pour $\sigma=\sigma_{0}$, la distance associée à cette norme sur $t_{G_{\sigma_{0}}}(\mathbf{C})$ est notée d. Soit $\mathcal{A} \rightarrow \operatorname{Spec} \mathcal{O}_{K}$ un modèle semi-abélien de $A$ sur une extension finie $K$ de $k$. Le couple $\overline{t_{\mathcal{A}}}=\left(t_{\mathcal{A}},\left(\|\cdot\|_{\sigma}\right)_{\sigma: K \hookrightarrow \mathbf{C}}\right)$ définit un fibré vectoriel hermitien sur Spec $\mathcal{O}_{K}$. En notant $\overline{\mathcal{W}_{0}}$ le sous-fibré hermitien induit par le module projectif $\mathcal{W}_{0}=\left(W_{0} \otimes_{k} K\right) \cap t_{\mathcal{A}}$, le nombre réel $\breve{h}\left(W_{0}\right)$ est défini comme la différence $\widehat{\operatorname{deg}_{n}} \overline{t_{\mathcal{A}}}-\widehat{\operatorname{deg}_{n}} \overline{\mathcal{W}_{0}}$. Ce nombre peut s'interpréter comme la différence des hauteurs de Schmidt de $W_{0}$ et $t_{A}$ (dans cet ordre), avec les métriques $\|\cdot\|_{\sigma}$ aux places infinies de $k$ et en prenant en compte la structure entière donnée par $t_{\mathcal{A}}$. Interviendra également dans le théorème 3.1 le terme $h \overline{\mathcal{O}_{X_{0}}(1)}\left(m u_{0}\right)$ qui correspond à la hauteur du point $1 \oplus m u_{0}$ de $X_{0}(k)$, en un sens que nous allons définir maintenant, au moyen des modèles $\overline{t_{\mathcal{A}}}$ et $\overline{\mathcal{W}_{0}}$. Tout d'abord, considérons le schéma projectif

$$
\pi_{0}: \mathcal{X}_{0}:=\mathbf{P}\left(\mathcal{O}_{K} \oplus\left(t_{\mathcal{A}} / \mathcal{W}_{0}\right)^{\vee}\right) \rightarrow \operatorname{Spec} \mathcal{O}_{K}
$$

de fibre générique $X_{0} \times_{\text {Spec } k}$ Spec $K$. Par somme directe hermitienne avec le fibré vectoriel hermitien trivial $\overline{\mathcal{O}_{K}}=\left(\mathcal{O}_{K},\left(\|1\|_{\sigma}=1\right)_{\sigma: K \hookrightarrow \mathbf{C}}\right)$, le $\mathcal{O}_{K}$-module projectif de type fini $\mathcal{O}_{K} \oplus\left(t_{\mathcal{A}} / \mathcal{W}_{0}\right)^{\vee}$ a une 
structure de fibré vectoriel hermitien $\operatorname{sur} \operatorname{Spec} \mathcal{O}_{K}$ (les métriques sur le dual du quotient sont celles induites par $\left.\overline{t_{\mathcal{A}}}\right)$. Le faisceau canonique $\mathcal{O}_{\mathcal{X}_{0}}(1)$ est un quotient de $\pi_{0}^{*}\left(\mathcal{O}_{K} \oplus\left(t_{\mathcal{A}} / \mathcal{W}_{0}\right)^{v}\right)$. Il hérite donc naturellement de métriques quotient — dites métriques de Fubini-Study — et ces dernières se transportent sur les fibres $x_{0}^{*} \mathcal{O}_{\mathcal{X}_{0}}(1) \otimes_{\sigma} \mathbf{C}$, pour tout $x_{0} \in \mathcal{X}_{0}\left(\mathcal{O}_{K}\right)$ et $\sigma: K \hookrightarrow \mathbf{C}$. De la sorte, nous avons défini un fibré en droites hermitien $\overline{x_{0}^{*} \mathcal{O}_{\mathcal{X}_{0}}(1)}$ sur $\operatorname{Spec} \mathcal{O}_{K}$, ce qui permet de définir la hauteur de $x_{0}$ comme le degré d'Arakelov normalisé de ce fibré :

$$
h \overline{\mathcal{O}_{X_{0}}(1)}\left(x_{0}\right):=\widehat{\operatorname{deg}}_{\mathrm{n}} \overline{x_{0}^{*} \mathcal{O}_{\mathcal{X}_{0}}(1)} .
$$

Cette définition s'étend aux points de $X_{0}(K)$ en les relevant à $\mathcal{X}_{0}\left(\mathcal{O}_{K}\right)$. En particulier le point correspondant à $1 \oplus m u_{0}$ a une hauteur qui est le nombre $h_{\overline{\mathcal{O}_{X_{0}}(1)}}\left(m u_{0}\right)$ que l'on cherchait à définir. À une fonction bornée près, il s'agit de la hauteur de Weil (logarithmique absolue) de $\left(1, m u_{0}\right)$ lorsque l'on exprime $u_{0}$ dans une base de $t_{A} / W_{0}$.

Soit

et

$$
12:=(10 g)^{4}(g+t) n
$$

$$
\text { 13: }:=2^{3 g+t} 3^{2 g} g !(g+t) !(2(g+t)+1)^{g}(g+t)^{2} .
$$

Ce sont des constantes qui interviennent dès le début de la démonstration, p. 30. Fixons un nombre réel positif $b$ vérifiant l'inégalité

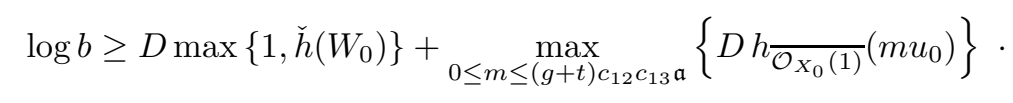

3.2. Hypothèses et données supplémentaires. Soit $\mathfrak{e} \geq e$ et $a_{1} \geq \cdots \geq a_{n}$ des nombres réels strictement positifs vérifiant

$$
\forall i \in\{1, \ldots, n\}, \quad \log a_{i} \geq \max \left\{\widehat{h}_{L_{i}}\left(p_{i}\right), \frac{\left(\mathfrak{e}\left\|u_{i}\right\|_{\sigma_{0}}\right)^{2}}{D}\right\} .
$$

Définissons alors l'entier a par

$$
\mathfrak{a}:=\left[\frac{D}{\log \mathfrak{e}} \max \left\{1, \log ^{+}\left(\frac{D}{\log \mathfrak{e}}\right), \mathrm{h}_{F}, \log ^{+} \log a_{1}\right\}\right]+1 .
$$

Dans l'énoncé du théorème 3.1 qui va suivre, et par conséquent dans toute la démonstration ( $(5)$, nous supposerons qu'une au moins des deux hypothèses suivantes est satisfaite :

(1) Pour tout entier $m \in\left\{1, \ldots, h_{12} \phi_{13} \mathfrak{a}\right\}$, pour tout sous-groupe algébrique connexe $G^{\prime}$ de $G_{\bar{k}}$ tel que $W_{\bar{k}}+t_{G^{\prime}} \neq t_{G_{\bar{k}}}$, le point $m p$ n'appartient pas à $G^{\prime}(\bar{k})$.

(2) Le sous-espace $W_{0}$ est un hyperplan $(i . e . t=1)$ et, pour tout entier $m \in\left\{1, \ldots, \phi_{12} \mid c_{13} \mathfrak{a}\right\}$, pour tout sous-groupe algébrique connexe et strict $G^{\prime}$ de $\{0\} \times A_{\bar{k}}$, le point $m p$ n'appartient pas à $G^{\prime}(\bar{k})$.

On notera que la seconde hypothèse implique la première puisque, lorsque $t=1$, un sous-groupe algébrique connexe $G^{\prime}$ de $G_{\bar{k}} \simeq \mathbb{G}_{\mathrm{a}, \bar{k}} \times A_{\bar{k}}$ qui vérifie $t_{G^{\prime}}+W_{\bar{k}} \neq t_{G_{\bar{k}}}$ est nécessairement un sous-groupe strict de $\{0\} \times A_{\bar{k}}$.

Définissons un entier $y \in\{0,1\}$ par

$$
y:= \begin{cases}1 & \text { si (1) est vérifié et (2) ne l'est pas, } \\ 0 & \text { si (2) est vérifié. }\end{cases}
$$

Introduisons ensuite un sous-espace vectoriel (quelconque) $\mathrm{V}$ de $\left(t_{A} / W_{0}\right) \otimes \bar{k}$ et notons $\overline{\mathrm{V}}:=$ $\mathbf{V}\left(\mathrm{V}^{\mathrm{v}}\right) \hookrightarrow G_{0, \bar{k}}$ le schéma en groupes affine sur $\bar{k}$ associé à $\mathrm{V}$ (l'espace tangent à l'origine de $\overline{\mathrm{V}}$ est $\mathrm{V}$ ). Soit $\mathrm{B}$ une sous-variété abélienne stricte de $A_{\bar{k}}$ et $\mathrm{H}:=\overline{\mathrm{V}} \times \mathrm{B}$ le groupe algébrique connexe produit. À de telles données $\mathrm{V}$ et $\mathrm{B}$, nous associons les entiers

$$
\varsigma_{\mathrm{H}}:=t-\operatorname{dim}\left(\mathrm{V}+\lambda\left(t_{\mathrm{B}}\right)\right), \quad t_{\mathrm{H}}:=\operatorname{dim} \mathrm{V}, \quad \varrho_{\mathrm{H}}:=\operatorname{dim}\left(\mathrm{V}+\lambda\left(t_{\mathrm{B}}\right)\right)-\operatorname{dim} \mathrm{V} .
$$

Définissons enfin l'entier $\delta_{\mathrm{B}}$ comme le plus grand entier $i$ de $\{1, \ldots, n\}$ tel que l'on ait $g_{n}+\cdots+g_{i}>$ $\operatorname{dim} B$. Nous supposons une fois pour toute que $\varsigma_{\mathrm{H}}>0$, c'est-à-dire $t_{\mathrm{H}}+W_{\bar{k}} \neq t_{G_{\bar{k}}}$. Le théorème 3.1. fait intervenir $\mathrm{H}$ comme un «paramètre ». Le choix «standard s sous-jacent à la plupart des énoncés présents dans la littérature est $\mathrm{H}=\{0\}$. 
3.3. Énoncé du théorème principal et conséquences. Nous sommes maintenant en mesure d'énoncer le théorème principal de ce texte, dont l'objectif est de fournir une minoration de la distance $\mathrm{d}\left(u, W_{\mathbf{C}}\right)$ du vecteur $u:=u_{0} \oplus \cdots \oplus u_{n} \in t_{G_{\sigma_{0}}}(\mathbf{C})$ (qui est un logarithme de $p$ ) à l'espace vectoriel $W \otimes_{\sigma_{0}} \mathbf{C}$.

Théorème 3.1. Avec les notations et hypothèses des $\S \S 3.1$ et 3.9, notons U le nombre réel défini par l'égalité

$$
\begin{aligned}
U^{\varsigma_{H}}:= & \left\{\prod_{j=1}^{\delta_{\mathrm{B}}-1}\left(1+\frac{D \mathfrak{a}}{\log \mathfrak{e}} \log a_{j}\right)^{g_{j}}\right\}\left(1+\frac{D \mathfrak{a}}{\log \mathfrak{e}} \log a_{\delta_{\mathrm{B}}}\right)^{g_{\delta_{\mathrm{B}}}+\cdots+g_{n}-\operatorname{dim} \mathrm{B}} \\
& \times \frac{\mathfrak{a} \operatorname{deg}_{\iota} \mathrm{B}}{(\mathfrak{a} \log \mathfrak{e})^{\varrho_{\mathrm{H}}}}\left(\log b+\mathfrak{a}^{y} \log \mathfrak{e}\right)^{t-\operatorname{dim} \vee}
\end{aligned}
$$

(si $\delta_{\mathrm{B}}=1$, le terme entre accolades vaut 1$)$. Alors u n'appartient pas à $W_{\mathbf{C}}$ et on a

$$
\log \mathrm{d}\left(u, W_{\mathbf{C}}\right) \geq-c_{5} U \quad \text { avec } \quad c_{5}:=(10(g+t))^{13(g+t) \frac{\operatorname{codim} H}{\varsigma_{H}}} .
$$

La première assertion du théorème, $u \notin W_{\mathbf{C}}$, est une conséquence du théorème du sous-groupe analytique de Wüstholz rappelé au début de l'introduction et de l'hypothèse (1) faite sur le point $p$.

La mesure (11) comporte les caractéristiques techniques relatives aux quantités $\log b$ et $\log a_{i}$, $1 \leq i \leq n$, déjà évoquées dans l'introduction (en prenant $\mathrm{H}=\{0\}$ ) et nous ne revenons pas dessus. Le théorème 3.1 revêt un aspect extrêmement général, ce qui le rend plus difficile à appréhender que les théorèmes 1.1 et 1.3 par exemple. L'explication de cette difficulté provient en partie du sous-groupe algébrique $\mathrm{H}=\overline{\mathrm{V}} \times \mathrm{B}$ qui intervient dans la définition de $U$. Dans les quelques lignes qui vont suivre, nous allons expliquer en quoi le caractère arbitraire de ce groupe — qui ne doit satisfaire qu'à la condition $t_{\mathrm{H}}+W_{\bar{k}} \neq t_{G_{\bar{k}}}$ - constitue une innovation par rapport aux mesures comparables déjà connues 23, 28] et se révéler utile pour d'éventuelles applications. Au même titre que le nombre réel $\mathfrak{e}$, le groupe $\mathrm{H}$ joue le rôle d'un paramètre dont la variation entraîne plusieurs minorations de $\log \mathrm{d}\left(u, W_{\mathbf{C}}\right)$, parfois inattendues et assez différentes entre elles. Avant de disséquer la mesure (11), rappelons que la conjecture de Lang-Waldschmidt énoncée (dans le cas linéaire seulement mais, a priori, généralisable au cas abélien) pages 212 et 213 de [42] prévoit l'existence d'une constante $c>0$, indépendantes des $\log a_{i}$ et de $\log b$, mais pouvant dépendre de $D$ et de $A$, telle que si $u \notin W_{\mathbf{C}}$ alors $\log \mathrm{d}\left(u, W_{\mathbf{C}}\right) \geq-c \log \left(b a_{1} \cdots a_{n}\right)$. Si la mesure (11) reste fondamentalement très éloignée de cette minoration, nous allons voir que l'on peut parfois s'en rapprocher un peu plus qu'avec la mesure obtenue en choisissant $H=\{0\}$.

Pour commencer*, regardons plus avant le cas $n=1$. Dans un premier temps, pour simplifier, supposons que le groupe $\mathrm{H}$ est tel que $\lambda\left(t_{\mathrm{B}}\right) \subseteq \mathrm{V}$. Dans ces conditions, on a $\delta_{\mathrm{B}}=1($ car $n=1)$, $\varsigma_{\mathrm{H}}=t-\operatorname{dim} \mathrm{V}$ et $\varrho_{\mathrm{H}}=0$. La mesure (11) devient alors

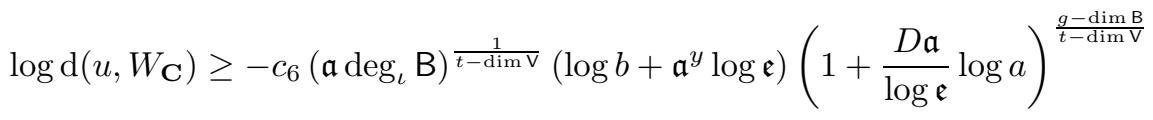

avec $c_{6}:=(10(g+t))^{13(g+t)(1+(g-\operatorname{dim} \mathrm{B}) /(t-\operatorname{dim} \mathrm{V}))}$. Sous cette forme, il ressort que le meilleur choix pour $\mathrm{V}$ est $\mathrm{V}=\lambda\left(t_{\mathrm{B}}\right)$ puisque le membre de droite décroît en fonction de $\operatorname{dim} \mathrm{V}$. Une fois cette observation faite, l'on peut vouloir minimiser $\lambda\left(t_{\mathrm{B}}\right)$ lui-même en supposant par exemple $t_{\mathrm{B}} \subseteq W_{0}$ (i.e. $\left.\lambda\left(t_{\mathrm{B}}\right)=\{0\}\right)$. Dans ce cas, la minoration (12) entraîne le

Corollaire 3.2. Avec les notations du $\S$ 3.1 avec $n=1$ et une des deux hypothèses sur le point $p$ émises au $\S$ 3.A, pour toute sous-variété abélienne $\mathrm{B}$ de A telle que $t_{\mathrm{B}} \subseteq W_{0}$, on a

$$
\log \mathrm{d}\left(u, W_{\mathbf{C}}\right) \geq-c_{7}\left(\mathfrak{a} \operatorname{deg}_{\iota} \mathrm{B}\right)^{\frac{1}{t}}\left(\log b+\mathfrak{a}^{y} \log \mathfrak{e}\right)\left(1+\frac{D \mathfrak{a}}{\log \mathfrak{e}} \log a\right)^{\frac{\operatorname{codim} \mathrm{B}}{t}}
$$

avec $c_{7}=(10(g+t))^{13(g+t)(1+\operatorname{codim} \mathrm{B} / t)}$ et $\operatorname{codim} \mathrm{B}=g-\operatorname{dim} \mathrm{B}$.

\footnotetext{
*Si le lecteur souhaite avoir des expressions plus simples des mesures qui vont suivre, il peut choisir dans tous les $\operatorname{cas} \mathfrak{e}=e$ et $y=1$.
} 
En choisissant $\mathrm{B}=\{0\}, u_{0}=0$ et $y=1$, nous retrouvons ainsi la mesure donnée dans le théorème 1.1. Nous constatons également que la mesure (13) avec B quelconque s'obtient à partir de celle avec $B=\{0\}$ simplement en multipliant le membre de droite par le facteur

$$
\frac{\operatorname{deg}_{\iota} \mathrm{B}}{\left((10(g+t))^{13(g+t)}\left(1+\frac{D \mathfrak{a}}{\log \mathfrak{e}} \log a\right)\right)^{\frac{\operatorname{dim} \mathrm{B}}{t}}} .
$$

Ce nombre réel est d'autant plus petit que $\operatorname{deg}_{\iota}$ B est petit et $\operatorname{dim} B$ est grand. Le « meilleur » choix (celui qui optimise l'inégalité (13)) ne correspond pas toujours nécessairement à $B=\{0\}$. Et c'est d'ailleurs ainsi que le théorème 1.3 se déduit du corollaire 3.2 en prenant $\mathrm{B}=B$ (nous reviendrons sur la preuve du théorème 1.3 page 52). Lorsque nous ne faisons plus l'hypothèse $t_{\mathrm{B}} \subseteq W_{0}$, nous avons un énoncé un peu plus général en prenant $\mathrm{H}=\lambda\left(t_{\mathrm{B}}\right) \times \mathrm{B}$, qui est valide pour toute sous-variété abélienne $\mathrm{B}$ telle que $W_{0}+t_{\mathrm{B}} \neq t_{A}$, et en remplaçant alors la puissance $1 / t$ par $1 /\left(g-\operatorname{dim}\left(W_{0}+t_{\mathrm{B}}\right)\right)$ dans (13) (ainsi que dans la constante $c_{7}$ ). Mais l'énoncé ainsi obtenu n'est pas fondamentalement plus général que le précédent puisque le corollaire 3.2 y conduit en changeant $W_{0}$ par $W_{0}+t_{\mathrm{B}}$.

Revenons maintenant au cas général $n \geq 1$. Le théorème 3.1 fournit des versions « produit 》 des énoncés précédents, qui séparent les contributions de chacune des composantes $p_{i}$ du point $p$. Par exemple, en choisissant $\mathrm{H}=\{0\}$, l'on déduit la minoration

$$
\log \mathrm{d}\left(u, W_{\mathbf{C}}\right) \geq-c_{8} \mathfrak{a}^{1 / t}\left(\log b+\mathfrak{a}^{y} \log \mathfrak{e}\right) \prod_{i=1}^{n}\left(1+\frac{D \mathfrak{a}}{\log \mathfrak{e}} \log a_{i}\right)^{g_{i} / t}
$$

avec $c_{8}:=(10(g+t))^{13 \frac{(g+t)^{2}}{t}}$. Cela généralise l'inégalité (2) du théorème 1.1. De même, pour toute sous-variété abélienne $B$ de $A$, de codimension $t \geq 1$, les choix $u_{0}=0, W_{0}=t_{B}$ et $\mathrm{H}=\{0\} \times B$ dans le théorème 3.1 conduisent à la généralisation suivante du théorème 1.3 (sous les mêmes hypothèses) :

$$
\begin{aligned}
\log \mathrm{d}\left(u, t_{B_{\sigma_{0}}}(\mathbf{C})\right) \geq- & c_{4}\left(\mathfrak{a} \operatorname{deg}_{\iota} B\right)^{1 / t}\left(\log b+\mathfrak{a}^{y} \log \mathfrak{e}\right) \prod_{j=1}^{\delta_{B}-1}\left(1+\frac{D \mathfrak{a}}{\log \mathfrak{e}} \log a_{j}\right)^{g_{j} / t} \\
& \times\left(1+\frac{D \mathfrak{a}}{\log \mathfrak{e}} \log a_{\delta_{B}}\right)^{\frac{g_{\delta_{B}}+\cdots+g_{n}-\operatorname{dim} B}{t}}
\end{aligned}
$$

qui, en utilisant la décroissance des $a_{i}$, devient plus simplement

$$
\log \mathrm{d}\left(u, t_{B_{\sigma_{0}}}(\mathbf{C})\right) \geq-c_{4}\left(\mathfrak{a} \operatorname{deg}_{\iota} B\right)^{1 / t}\left(\log b+\mathfrak{a}^{y} \log \mathfrak{e}\right)\left(1+\frac{D \mathfrak{a}}{\log \mathfrak{e}} \log a_{1}\right)
$$

formellement identique à l'inégalité (5) du théorème 1.3 (la constante $c_{4}$ est définie dans ce théorème).

\section{Outils auxiliaires}

La démonstration du théorème 3.1 requiert un certain nombre de résultats annexes disséminés dans la littérature et, parfois, présentés sous une forme assez éloignée de celle voulue ici. C'est pourquoi nous les avons rassemblés dans une partie indépendante du reste du texte.

Nous supposerons que le lecteur est familier avec la notion de fibré vectoriel hermitien sur $\operatorname{Spec} \mathcal{O}_{k}(k$ corps de nombres). L'on pourra se référer au début de l'appendice de [10] ou au $\S 4.1$ de 12. pour un exposé systématique de cette notion et de certaines propriétés qui l'accompagnent.

4.1. Puissances symétriques. Soit $\overline{\mathcal{E}}=\left(\mathcal{E},\left(\|\cdot\|_{\sigma}\right)_{\sigma: k \hookrightarrow \mathbf{C}}\right)$ un fibré vectoriel hermitien $\operatorname{sur} \operatorname{Spec} \mathcal{O}_{k}$ de rang $N+1$ et $\ell$ un entier strictement positif. Étant donné une place finie $\mathfrak{p}$ de $k$ d'anneau de valuation $\mathcal{O}_{\mathfrak{p}}$, le module $\mathcal{E}_{\mathfrak{p}}:=\mathcal{E} \otimes \mathcal{O}_{\mathfrak{p}}$ est libre de rang $N+1$. En considérant une base $\left(e_{0}, \ldots, e_{N}\right)$ $\operatorname{sur} \mathcal{O}_{\mathfrak{p}}$ de $\mathcal{E}_{\mathfrak{p}}$, la norme $\mathfrak{p}$-adique $\|\cdot\|_{\overline{\mathcal{E}}, \mathfrak{p}}$ sur $\mathcal{E}_{\mathfrak{p}}$ est définie par $\left\|\sum_{i=0}^{N} x_{i} e_{i}\right\|_{\overline{\mathcal{E}}, \mathfrak{p}}=\max _{0 \leq i \leq N}\left\{\left|x_{i}\right|_{\mathfrak{p}}\right\}$, où la valeur absolue $\mathfrak{p}$-adique $|\cdot|_{\mathfrak{p}}$ sur $\mathcal{O}_{\mathfrak{p}}$ est normalisée par $|p|_{\mathfrak{p}}=p^{-1}, p$ étant la caractéristique résiduelle de $\mathfrak{p}$.

Pour chaque plongement complexe $\sigma: k \hookrightarrow \mathbf{C}$, nous munissons la ème puissance symétrique $S^{\ell}\left(\mathcal{E}_{\sigma}\right)$ de la métrique quotient $\|\cdot\|_{\ell, \sigma}$ déduite de la projection canonique $\mathcal{E}_{\sigma}^{\otimes \ell} \rightarrow S^{\ell}\left(\mathcal{E}_{\sigma}\right)$. Soit 
$\left(e_{0}, \ldots, e_{N}\right)$ une base de $\mathcal{E}_{k}$ et $\left(e_{0}^{*}, \ldots, e_{N}^{*}\right)$ sa base duale. Si $\left(e_{0}, \ldots, e_{N}\right)$ est une base orthonormale de $\mathcal{E}_{\sigma}$, le carré de la norme d'un élément e $:=\sum x_{\mathbf{i}} e_{0}^{i_{0}} \cdots e_{N}^{i_{N}}$ vaut

$$
\|\mathrm{e}\|_{\ell, \sigma}^{2}=\sum_{|\mathbf{i}|=\ell}\left|x_{\mathbf{i}}\right|^{2} \frac{\mathbf{i} !}{\ell !} .
$$

Il y a un isomorphisme naturel $\Theta_{\ell}$ entre $S^{\ell}\left(\mathcal{E}^{\vee}\right) \otimes k$ et $\left(S^{\ell}(\mathcal{E})\right)^{\vee} \otimes k$ qui fait correspondre le vecteur

$$
\mathbf{a}:=\sum_{|\mathbf{i}|=\ell} a_{\mathbf{i}} e_{0}^{* i_{0}} \cdots e_{N}^{* i_{N}}
$$

de $S^{\ell}\left(\mathcal{E}_{k}^{\vee}\right)$ à la forme linéaire

$$
\sum_{\substack{\mathbf{i} \in \mathbf{N}^{N+1} \\|\mathbf{i}|=\ell}} x_{\mathbf{i}} e_{0}^{i_{0}} \cdots e_{N}^{i_{N}} \mapsto \sum_{\mathbf{i}} a_{\mathbf{i}} x_{\mathbf{i}} \quad \operatorname{de} S^{\ell}\left(\mathcal{E}_{k}\right) .
$$

Lemme 4.1. L'application $k$-linéaire $\Theta_{\ell}$ est une isométrie aux places ultramétriques de $k$. Pour tout $\sigma: k \hookrightarrow \mathbf{C}$, les normes d'opérateurs de $\Theta_{\ell}$ et $\Theta_{\ell}^{-1}$ satisfont

$$
\left\|\Theta_{\ell}\right\|_{\sigma} \leq \max _{\substack{\mathbf{i} \in \mathbf{N}^{N+1}|\mathbf{i}|=\ell \\ \text { in }}}\left\{\frac{\ell !}{\mathbf{i} !}\right\} \quad \text { et } \quad\left\|\Theta_{\ell}^{-1}\right\|_{\sigma} \leq 1 .
$$

Démonstration. En une place finie $\mathfrak{p}$, la première assertion est une conséquence immédiate du choix de la norme sur $\mathcal{E}_{\mathfrak{p}}$. Si $\sigma$ est un plongement complexe de $k$, il faut utiliser l'inégalité de Cauchy-Schwarz

$$
\left|\sum_{\mathbf{i}} a_{\mathbf{i}} x_{\mathbf{i}}\right|=\left|\sum_{\mathbf{i}}\left(a_{\mathbf{i}} \sqrt{\frac{\mathbf{i} !}{\ell !}}\right)\left(x_{\mathbf{i}} \sqrt{\frac{\mathbf{i} !}{\ell !}}\right) \frac{\ell !}{\mathbf{i} !}\right| \leq\left(\max _{\substack{\mathbf{i} \in \mathbf{N}^{N+1}|\mathbf{i}|=\ell \\ \text { in }}}\left\{\frac{\ell !}{\mathbf{i} !}\right\}\right)\|\mathbf{a}\|_{\sigma}\|\mathbf{x}\|_{\sigma} .
$$

La preuve est la même pour $\Theta_{\ell}^{-1}$ en remarquant que $\max _{\substack{\mathbf{i} \in \mathbf{N} N+1 \\ \mathbf{i} \mid=\ell}}\left\{\frac{\mathbf{i} !}{\ell !}\right\}=1$.

4.2. Métriques. Dans ce paragraphe, nous explicitons les métriques que nous utiliserons dans la preuve du théorème 3.1 (voir les estimations analytiques du $\S 5.9$ ).

4.2.1. Métriques de Fubini-Study. Soit $\overline{\mathcal{E}}$ un fibré vectoriel hermitien sur $\mathcal{O}_{k}$, de $\operatorname{rang} N+1$, et soit $\mathcal{O}(1)=\mathcal{O}_{\mathbf{P}(\mathcal{E})}(1)$ le fibré en droites canonique sur $\pi: \mathbf{P}(\mathcal{E}) \rightarrow \operatorname{Spec} \mathcal{O}_{k}$. Lorsque $\sigma$ est une plongement complexe de $k$, nous munissons $\mathcal{O}_{\mathbf{P}\left(\mathcal{E}_{\sigma}\right)}(1)$ de la métrique quotient provenant de $\pi_{\sigma}^{*}\left(\mathcal{E}_{\sigma}\right)$ (métrique de Fubini-Study). Soit $m$ un entier strictement positif, $x \in \mathbf{P}\left(\mathcal{E}_{\sigma}\right)(\mathbf{C})$ et $s \in \mathrm{H}^{0}\left(\mathbf{P}\left(\mathcal{E}_{\sigma}\right), \mathcal{O}(m)\right) \simeq S^{m}\left(\mathcal{E}_{\sigma}\right)$. Fixons également une base orthonormale de $\mathcal{E}_{\sigma}$. La section $s$ correspond à un polynôme complexe $P$, homogène en $N+1$ variables de degré $m$, et le point $x$ peut s'exprimer en termes de coordonnées $\left(x_{0}: \cdots: x_{N}\right) \in \mathbf{P}^{N}(\mathbf{C})$. L'invariance sous l'action du groupe unitaire $\mathbf{U}_{N+1}(\mathbf{C})$ de la forme de courbure de la métrique de Fubini-Study entraîne l'égalité

$$
\|s(x)\|_{\overline{\mathcal{O}(m)_{x}}, \sigma}=\frac{\left|P\left(x_{0}, \ldots, x_{N}\right)\right|}{\left(\left|x_{0}\right|^{2}+\cdots+\left|x_{N}\right|^{2}\right)^{m / 2}} .
$$

4.2.2. Métriques cubistes. De même que précédemment, la métrique cubiste $\|\cdot\|_{1}$ sur un fibré en droites ample $L$ au-dessus d'une variété abélienne complexe $A$ peut être décrite de manière concrète en utilisant les fonctions thêta. En effet, un résultat de Moret-Bailly (voir [51, Proposition 2.1, p. 48) affirme que la métrique cubiste est caractérisée par les propriétés suivantes.

1) Sa forme de courbure est invariante par translation,

2) la rigidification à l'origine $0^{*} \mathrm{~L} \simeq \mathcal{O}_{\text {Spec } \mathbf{C}}$ est une isométrie (avec la métrique triviale $\|1\|=1$ $\left.\operatorname{sur} \mathcal{O}_{\text {Spec }} \mathbf{C}\right)$.

D'autre part, l'espace des sections globales $H^{0}(A, L)$ peut être décrit en termes de fonctions thêta associées à une donnée d'Appel-Humbert $\left(\chi, H_{\mathrm{L}}\right)$ pour $\mathrm{L}$ (ici $H_{\mathrm{L}}$ est le produit scalaire hermitien qui provient de la première classe de Chern $c_{1}(\mathrm{~L})$, voir [5] pour plus de détails). Étant donné une section $s$ de $\mathrm{L}$ et $\vartheta$ la fonction thêta associée à $s$, on vérifie que la forme de courbure de la métrique $\|\cdot\|_{2}$ définie par

$$
\|s(x)\|_{2}:=e^{-\frac{\pi}{2} H_{\mathrm{L}}(z, z)}|\vartheta(z)| \quad\left(x:=\exp _{\mathrm{A}}(z), z \in t_{\mathrm{A}}\right)
$$


est invariante par translation (et triviale à l'origine). D'après le résultat de Moret-Bailly, les deux métriques $\|\cdot\|_{1}$ et $\|\cdot\|_{2}$ sont les mêmes.

4.3. Calculs de pentes d'Arakelov. Soit $\overline{\mathcal{E}}=\left(\mathcal{E},\left(<,>_{\sigma}\right)_{\sigma: k \hookrightarrow \mathbf{C}}\right)$ un fibré vectoriel hermitien sur Spec $\mathcal{O}_{k}$ de rang $N+1$ (ici $<,>_{\sigma}$ désigne le produit hermitien sur $\mathcal{E}_{\sigma}$ et non la norme associée). La puissance extérieure maximale $\operatorname{det} \mathcal{E}=\bigwedge^{N+1} \mathcal{E}$ hérite d'une structure hermitienne aux places infinies $\sigma$ de $k$ en considérant les normes définies par

$$
\forall x_{0}, \ldots, x_{N} \in \mathcal{E}_{\sigma},\left\|x_{0} \wedge \cdots \wedge x_{N}\right\|_{\operatorname{det} \overline{\mathcal{E}}, \sigma}^{2}:=\operatorname{det}\left(<x_{i}, x_{j}>_{\sigma}\right)_{0 \leq i, j \leq N} .
$$

Soit $s \in(\operatorname{det} \mathcal{E}) \backslash\{0\}$. Le degré d'Arakelov normalisé de $\overline{\mathcal{E}}$ est

$$
\widehat{\operatorname{deg}}_{\mathrm{n}} \overline{\mathcal{E}}:=\frac{1}{[k: \mathbf{Q}]}\left(\log \operatorname{card}\left(\operatorname{det} \mathcal{E} / s . \mathcal{O}_{k}\right)-\sum_{\sigma: k \hookrightarrow \mathbf{C}} \log \|s\|_{\operatorname{det} \overline{\mathcal{E}}, \sigma}\right) .
$$

On vérifie avec la formule du produit que cette expression ne dépend pas du choix de $s$. Par convention, si $\mathcal{E}=\{0\}$, on pose $\widehat{\operatorname{deg}}_{n} \overline{\mathcal{E}}=0$. La pente d'A rakelov normalisée de $\overline{\mathcal{E}}$ est le quotient du degré d'Arakelov normalisé par son rang :

$$
\widehat{\mu}(\overline{\mathcal{E}}):=\frac{\widehat{\operatorname{deg}}_{\mathrm{n}} \overline{\mathcal{E}}}{\operatorname{rg}(\mathcal{E})} .
$$

Cette application transforme les produits tensoriels en sommes : $\widehat{\mu}(\overline{\mathcal{E}} \otimes \overline{\mathcal{F}})=\widehat{\mu}(\overline{\mathcal{E}})+\widehat{\mu}(\overline{\mathcal{F}})$. Nous allons calculer ici la pente de certains fibrés hermitiens provenant de la géométrie algébrique. Mais auparavant, rappelons que la pente maximale $\widehat{\mu}_{\max }(\overline{\mathcal{E}})$ est le maximum des pentes d'Arakelov $\widehat{\mu}\left(\overline{\mathcal{E}^{\prime}}\right)$ lorsque $\overline{\mathcal{E}^{\prime}}$ parcourt les sous-fibrés vectoriels hermitiens de $\overline{\mathcal{E}}$. Et, là encore, il est commode, lorsque $\mathcal{E}=\{0\}$, de poser $\widehat{\mu}(\overline{\mathcal{E}})=\widehat{\mu}_{\max }(\overline{\mathcal{E}})=-\infty$. Au $\S 4.5$, nous regarderons plus en détail les relations entre ces pentes lorsque $\overline{\mathcal{E}}$ varie.

4.3.1. Reprenons le fibré vectoriel hermitien $\overline{\mathcal{E}}$ ci-dessus. Nous munissons le fibré en droites canonique $\mathcal{O}_{\mathbf{P}(\mathcal{E})}(1)$ de la métrique de Fubini-Study aux places infinies de $k$. Ainsi, pour tout entier $m \geq 1$, le fibré en droites $\mathcal{O}_{\mathbf{P}(\mathcal{E})}(m):=\mathcal{O}_{\mathbf{P}(\mathcal{E})}(1)^{\otimes m}$ est métrisé et il y a une structure de fibré vectoriel hermitien sur le faisceau des sections globales $\pi_{*} \mathcal{O}_{\mathbf{P}(\mathcal{E})}(m)$ (voir égalité (36)). Par exemple, si $s \in \pi_{*} \mathcal{O}_{\mathbf{P}\left(\mathcal{E}_{\sigma}\right)}(m)$ et si $\left(p_{\mathbf{i}}\right)_{|\mathbf{i}|=m}$ désignent les coefficients du polynôme $P$ associé à $s$ (dans la base des monômes, voir $\S 4.2 .1$ ), nous avons

$$
\|s\|_{\frac{2}{\mathcal{O}(m)}, \sigma}=\sum_{\substack{\mathbf{i} \in \mathbf{N}^{N+1} \\|\mathbf{i}|=m}}\left|p_{\mathbf{i}}\right|^{2} \frac{N ! \mathbf{i} !}{(N+m) !} .
$$

On peut noter que ces dernières métriques sont celles sur $\overline{S^{m}(\mathcal{E})}$ (définie au $\S 4.1$ ), multipliées $\operatorname{par}\left(\begin{array}{c}m+N \\ m\end{array}\right)^{-1 / 2}$. Le résultat suivant est une variante de la proposition 4.2 .8 de la thèse de Randriambololona [59].

Proposition 4.2. Notons $\gamma_{N, m}$ le nombre réel

$$
\left\{\prod_{\substack{\mathbf{i} \in \mathbf{N} N+1 \\
|\mathbf{i}|=m}}\left(\begin{array}{c}
m \\
\mathbf{i}
\end{array}\right)\right\}^{\frac{1}{\left(\begin{array}{c}
m+N \\
m
\end{array}\right)}} .
$$

La pente d'Arakelov normalisée du fibré vectoriel hermitien $\overline{\mathrm{H}^{0}\left(\mathbf{P}(\mathcal{E}), \mathcal{O}_{\mathbf{P}(\mathcal{E})}(m)\right)}$ est

$$
\widehat{\mu}\left(\overline{\mathrm{H}^{0}\left(\mathbf{P}(\mathcal{E}), \mathcal{O}_{\mathbf{P}(\mathcal{E})}(m)\right)}\right)=\frac{1}{2} \log \left\{\left(\begin{array}{c}
m+N \\
m
\end{array}\right) \gamma_{N, m}\right\}+m \widehat{\mu}(\overline{\mathcal{E}}) .
$$

Esquisse de la preuve. Après une éventuelle extension (finie) de corps (ce qui ne modifie pas les pentes normalisées), nous pouvons supposer que $\mathcal{E}$ est un $\mathcal{O}_{k}$-module libre. Choisissons un isomorphisme $q: \mathcal{O}_{k}^{N+1} \rightarrow \mathcal{E}$. En munissant $\mathcal{O}_{k}^{N+1}$ des métriques «triviales » aux places infinies de $k$, nous pouvons évaluer les normes archimédiennes de la $m^{\text {ème }}$ puissance symétrique $S^{m} q$ : 
$S^{m}\left(\mathcal{O}_{k}^{N+1}\right) \stackrel{\sim}{\rightarrow} S^{m}(\mathcal{E})$, à partir de laquelle nous déduisons la pente de $\overline{S^{m}(\mathcal{E})}$ (à savoir, $\widehat{\mu}\left(\overline{S^{m} \mathcal{E}}\right)=$ $\left.m \widehat{\mu}(\overline{\mathcal{E}})+\frac{1}{2} \log \gamma_{N, m}\right)$. La formule $(19)$ s'ensuit via l'isomorphisme

$$
S^{m}(\mathcal{E}) \simeq \mathrm{H}^{0}\left(\mathbf{P}(\mathcal{E}), \mathcal{O}_{\mathbf{P}(\mathcal{E})}(m)\right)
$$

(l'on contrôle le changement de normes au moyen de (15) et (18)). Des détails supplémentaires sont donnés dans op. cit.

4.3.2. Un autre calcul de pente concerne le fibré des sections globales d'un fibré en droites ample et symétrique $L$ au-dessus d'une variété abélienne $A$ sur un corps de nombres $k$. Avant cela, il faut définir une structure de fibré vectoriel hermitien sur $\mathrm{H}^{0}(A, L)$.

Définition-Théorème 4.3. Soit $(A, L)$ comme ci-dessus et $\Sigma$ un sous-ensemble fini de $A(k)$. Le triplet $(A, L, \Sigma)$ possède un modèle de Moret-Bailly $(\mathcal{A}, \overline{\mathcal{L}}, \Sigma)$ au sens (restreint) suivant. Il existe une extension finie $K \mid k$ et un schéma en groupes $\mathcal{A} \rightarrow \operatorname{Spec} \mathcal{O}_{K}$ semi-stable (donc lisse), de fibre générique isomorphe à $A_{K}$. Il existe un fibré hermitien cubiste $\overline{\mathcal{L}} \rightarrow \mathcal{A}$, de fibre générique $L_{K}$ (le terme cubiste signifie ici qu'aux places infinies de $K$ la métrique sur $\mathcal{L} \otimes_{\sigma} \mathbf{C}$ est celle définie au $\S 4.2 .2)$. Et enfin, tout élément $q$ de $\Sigma$ se relève en une section $\varepsilon_{q} \in \mathcal{A}\left(\mathcal{O}_{K}\right)$.

L'existence de telles données est démontrée au $\S 4.3$ de [9]. En réalité, un modèle de Moret-Bailly tel qu'il est défini dans cet article possède d'autres propriétés (relatives en particulier au groupe de Mumford de $L^{\otimes 2}$ ) et qui le caractérisent plus finement. Et bien que nous ne les utiliserons pas explicitement dans ce texte, ces propriétés sont néanmoins sous-jacentes à la démonstration de la formule (20) qui va suivre. Le $\mathcal{O}_{K}$-module projectif $\mathrm{H}^{0}(\mathcal{A}, \mathcal{L})$ est muni aux places infinies de $K$ des métriques hermitiennes induites par les métriques de $\overline{\mathcal{L}}$ (voir la formule (36), p. 20, où la mesure $\mu_{\sigma}$ qui intervient dans cette formule est ici la mesure de Haar sur $\left.A_{\sigma}(\mathbf{C})\right)$.

Théorème 4.4 (Moret-Bailly, Bost). La pente d'Arakelov normalisée du fibré vectoriel hermitien $\overline{\mathrm{H}^{0}(\mathcal{A}, \mathcal{L})}$ est

$$
\widehat{\mu}\left(\overline{\mathrm{H}^{0}(\mathcal{A}, \mathcal{L})}\right)=-\frac{1}{2} h_{F}(A)+\frac{1}{4} \log \frac{\mathrm{h}^{0}(A, L)}{(2 \pi)^{g}}
$$

$(g=\operatorname{dim} A)$.

Remarque 4.5. Comme $L$ est ample, la dimension de $\mathrm{h}^{0}(A, L)$ est aussi la caractéristique d'EulerPoincaré $\chi(A, L)$ de $L$. Par conséquent, pour tout entier $m \geq 1$, nous avons $\mathrm{h}^{0}\left(A, L^{\otimes m}\right)=$ $m^{g} \mathrm{~h}^{0}(A, L)$ et la pente d'Arakelov de $\overline{\mathrm{H}^{0}\left(\mathcal{A}, \mathcal{L}^{\otimes m}\right)}$ s'obtient en ajoutant $\frac{g}{4} \log (m)$ au membre de droites de (20).

Le théorème 4.4 a été établie par Bost (théorème 4.10, (v), de [9]). Elle repose sur les travaux de Moret-Bailly [51, 52] et sur le théorème de Riemann-Roch arithmétique de Gillet et Soulé [31] lorsque $A$ a bonne réduction (sous cette hypothèse supplémentaire sur $A$ mais sans l'hypothèse de symétrie de $L$, il existe une formule exacte un peu plus compliquée pour la pente de $\overline{\mathrm{H}^{0}(\mathcal{A}, \mathcal{L})}$ (voir (4.1.3) dans 99)).

4.3.3. Soit $K$ une extension finie de $k$ sur laquelle $A$ est définie et admet réduction semi-abélienne. Soit $\pi: \mathcal{A} \rightarrow \operatorname{Spec} \mathcal{O}_{K}$ un modèle semi-abélien de $A$ et $\varepsilon: \operatorname{Spec} \mathcal{O}_{K} \rightarrow \mathcal{A}$ sa section nulle. Notons $\omega_{\mathcal{A} / \mathcal{O}_{K}}$ le faisceau inversible $\varepsilon^{*} \Omega_{\mathcal{A} / \operatorname{Spec} \mathcal{O}_{K}}^{g} \operatorname{sur} \mathcal{A}$. Ce fibré devient un fibré en droites hermitiens $\bar{\omega}_{\mathcal{A} / \mathcal{O}_{K}}$ sur $\operatorname{Spec} \mathcal{O}_{K}$ lorsqu'on le munit pour chaque plongement complexe $\sigma: K \hookrightarrow \mathbf{C}$ de la norme

$$
\forall s \in \omega_{\mathcal{A} / \mathcal{O}_{K}} \otimes_{\sigma} \mathbf{C} \simeq \mathrm{H}^{0}\left(A_{\sigma}(\mathbf{C}), \Omega_{A_{\sigma}(\mathbf{C})}^{g}\right), \quad\|s\|_{\bar{\omega}_{\mathcal{A} / \mathcal{O}_{K}}, \sigma}^{2}:=\frac{i^{g^{2}}}{(2 \pi)^{g}} \int_{A_{\sigma}(\mathbf{C})} s \wedge \bar{s} .
$$

Définition 4.6. La hauteur de Faltings (stable normalisée) $h_{F}(A)$ de $A$ est le degré d'Arakelov normalisé de $\bar{\omega}_{\mathcal{A} / \mathcal{O}_{K}}$.

Cette définition est indépendante des choix de $K$ et de $\mathcal{A}$. Bost a montré que

$$
h_{F}(A) \geq-\frac{g \log (2 \pi)}{2}
$$


(voir le corollaire 2 de $|\beta|$ ). De plus, un résultat de Raynaud affirme que si $\varphi: A \rightarrow A^{\prime}$ est une isogénie alors

$$
h_{F}\left(A^{\prime}\right) \leq h_{F}(A)+\frac{1}{2} \log \operatorname{deg} \varphi
$$

(corollaire 2.1.4 de [61]). Par ailleurs, le fibré en droites $\omega_{\mathcal{A} / \mathcal{O}_{K}}$ s'identifie au déterminant $\operatorname{det} t_{\mathcal{A}}^{v}$. Dans l'introduction, nous avons construit des normes hermitiennes sur les espaces $t_{\mathcal{A}_{\sigma}}(\mathbf{C})$ qui font de $t_{\mathcal{A}}$ un fibré vectoriel hermitien sur $\operatorname{Spec} \mathcal{O}_{K}$. Ces métriques se transmettent alors à $\omega_{\mathcal{A} / \mathcal{O}_{K}}$ par dualité et puissance extérieure maximale. Par définition, le degré d'Arakelov normalisée du fibré hermitien obtenu de la sorte est le degré de $\overline{t_{\mathcal{A}}^{v}}$. L'énoncé suivant précise la différence avec le degré $\operatorname{de} \bar{\omega}_{\mathcal{A} / \mathcal{O}_{K}}$.

Proposition 4.7 (Proposition D.1 de [10]). La pente d'Arakelov normalisée du fibré vectoriel hermitien dual $\overline{t_{\mathcal{A}}^{v}}$ est

$$
\widehat{\mu}\left(\overline{t_{\mathcal{A}}^{\vee}}\right)=\frac{h_{F}(A)}{g}+\frac{1}{2 g} \log h^{0}(A, L)-\frac{1}{2} \log \pi .
$$

Démonstration. Il suffit de montrer que, pour tout $\sigma: K \hookrightarrow \mathbf{C}$ et tout $s \in\left(\omega_{\mathcal{A} / \mathcal{O}_{K}}\right)_{\sigma} \backslash\{0\}$, on a

$$
\|s\|_{\operatorname{det} \overline{t_{\mathcal{A}}}, \sigma}=\left(\frac{\pi^{g}}{\mathrm{~h}^{0}(A, L)}\right)^{1 / 2}\|s\|_{\bar{\omega}_{\mathcal{A} / \mathcal{O}_{K}}, \sigma} .
$$

Soit $\left(e_{1}, \ldots, e_{g}\right)$ une base orthonormée de $t_{A_{\sigma}}(\mathbf{C})$ et $\left(e_{1}^{*}, \ldots, e_{g}^{*}\right)$ la base duale. Posons $s:=e_{1}^{*} \wedge$ $\cdots \wedge e_{g}^{*}$. Comme $\operatorname{dim}_{\mathbf{C}} \omega_{\mathcal{A}_{\sigma} / \mathbf{C}}=1$, il suffit de prouver (24) pour cet $s$ particulier. Dans la base orthonormée $\left(e_{1}^{*}, \ldots, e_{g}^{*}\right)$, la $(1,1)$-forme $\omega$, invariante par translation, qui représente la classe de Chern $c_{1}\left(L_{\sigma}\right)$, s'écrit $\omega=\frac{i}{2} \sum_{h=1}^{g} e_{h}^{*} \wedge \overline{e_{h}^{*}}$. En particulier, on a

$$
\omega^{g}=\left(\frac{i}{2}\right)^{g} \sum_{\eta}\left(e_{\eta(1)}^{*} \wedge \overline{e_{\eta(1)}^{*}}\right) \wedge \cdots \wedge\left(e_{\eta(g)}^{*} \wedge \overline{e_{\eta(g)}^{*}}\right)
$$

où $\eta$ parcourt les permutations de $\{1, \ldots, g\}$. Ainsi, $\omega^{g}= \pm\left(\frac{i}{2}\right)^{g} g ! s \wedge \bar{s}$ et en intégrant sur $A_{\sigma}(\mathbf{C})$, le membre de gauche donne

$$
\int_{A_{\sigma}(\mathbf{C})} \omega^{g}=\operatorname{deg}_{L_{\sigma}} A_{\sigma}=\operatorname{deg}_{L} A
$$

et le membre de droite fait apparaître $\frac{(2 \pi)^{g}}{i^{g^{2}}}\|s\|_{\omega_{\mathcal{A} / \mathcal{O}_{K}, \sigma}}$. En prenant le module et en utilisant la formule de Frobenius (7), nous obtenons l'égalité

$$
\|s\|_{\varpi_{\mathcal{A} / \mathcal{O}_{K}, \sigma}}=\left(\frac{\mathrm{h}^{0}(A, L)}{\pi^{g}}\right)^{1 / 2} .
$$

Pour en déduire l'égalité (24), il ne reste plus qu'à observer

$$
\|s\|_{\operatorname{det} \overline{t_{\mathcal{A}}}, \sigma}^{2}=\operatorname{det}\left(<e_{i}^{*}, e_{j}^{*}>\overline{t_{\mathcal{A}}^{v}, \sigma}\right)_{1 \leq i, j \leq g}=1
$$

car la base $\left(e_{1}^{*}, \ldots, e_{g}^{*}\right)$ est orthonormée. Pour conclure, il suffit de prendre le logarithme de (24) pour tous les plongements complexes $\sigma$ puis de sommer et d'utiliser les définitions du degré et de la pente d'Arakelov normalisés.

Comme nous allons le voir dans le paragraphe suivant, cet énoncé s'interprète aussi comme un calcul de hauteur.

4.4. Hauteurs. Tout au long de ce texte apparaissent des fonctions hauteurs appliquées à divers objets tels des espaces vectoriels, des applications $k$-linéaires ou bien encore des points algébriques de variétés arithmétiques. Nous allons rappeler les définitions et conventions choisies dans la suite de ce texte. Pour une vue plus globale du sujet, le lecteur intéressé pourra consulter par exemple [7, 13]. 
4.4.1. Conservons les notations du $\S 4.3 .2$ (dont, en particulier, le modèle de Moret-Bailly $(\mathcal{A}, \overline{\mathcal{L}})$ de $(A, L)$ au-dessus de $K)$. Soit $V$ un sous-espace vectoriel de $t_{A}(K)$ (de dimension $d$ ). Désignons par $\mathcal{V}:=V \cap t_{\mathcal{A}}$ le $\mathcal{O}_{K}$-module saturé obtenu à partir de $V$. Cela définit un sous-fibré vectoriel de $t_{\mathcal{A}}$ et, par définition, la hauteur projective $\breve{h}(V)$ de $V$ (relative à $(A, L))$ est le degré d'Arakelov normalisé du fibré vectoriel quotient $\overline{t_{\mathcal{A}} / \mathcal{V}}$, où $\mathcal{V}$ est muni des métriques de $\overline{t_{\mathcal{A}}}$ induites par restriction. La terminologie « hauteur projective » provient d'un résultat de Bost, Gillet \& Soulé, qui affirme que $\breve{h}(V)$ s'interprète comme la hauteur (normalisée) du cycle $\mathbf{P}\left(\mathcal{V}^{\mathrm{v}}\right)$ relative au fibré vectoriel hermitien $\overline{t_{\mathcal{A}}}$ (voir [13], $\S$ 4.1). La hauteur projective diffère de la hauteur de $V$, utilisée par Bost dans 10] et définie par $h(V):=\widehat{\operatorname{deg}}_{\mathrm{n}} \overline{\mathcal{V}^{\mathrm{v}}}$. Plus précisément, de la suite exacte courte de fibrés vectoriels hermitiens

$$
0 \rightarrow \overline{\mathcal{V}} \rightarrow \overline{t_{\mathcal{A}}} \rightarrow \overline{t_{\mathcal{A}} / \mathcal{V}} \rightarrow 0
$$

l'on déduit la relation

$$
\check{h}(V)=h(V)+\widehat{\operatorname{deg}}_{\mathrm{n}} \overline{t_{\mathcal{A}}}=h(V)-h\left(t_{A}\right) .
$$

En considérant le plongement de Plücker

$$
V=\operatorname{Vect}\left(v_{1}, \ldots, v_{d}\right) \mapsto\left[v_{1} \wedge \cdots \wedge v_{d}\right] \in \mathbf{P}\left(\bigwedge^{d} t_{A}(K)\right)
$$

et la hauteur de Weil sur $\mathbf{P}\left(\bigwedge^{d} t_{A}(K)\right)$, on vérifie que la hauteur projective est bien une « fonction hauteur » sur les sous-espaces de $t_{A}(K)$, avatar intrinsèque de la hauteur de Schmidt [63]. Soulignons que la quantité $h(V)$ dépend de $(A, L)$ et c'est pourquoi l'on peut employer la notation plus précise $h_{A, L}(V)$ au lieu de $h(V)$. Cela permet de lever toute ambiguïté lorsque $V$ peut être vu comme sous-espace de deux espaces tangents de variétés abéliennes distinctes.

Le principal intérêt de la hauteur projective par rapport à la hauteur réside dans le fait qu'elle est la quantité naturelle qui apparaît dans la démonstration du théorème 3.1. Notons aussi qu'en terme de pente l'on a $h\left(t_{A}\right)=g \widehat{\mu}\left(\overline{t_{A}^{v}}\right)$. La formule (25) est un moyen commode pour calculer explicitement la hauteur d'un sous-espace à l'aide d'équations qui le définissent dans une base de $t_{A}$. Nous en verrons une application au $\S 6$, lorsque $A$ est un produit de courbes elliptiques.

Lorsque $V$ est l'algèbre de Lie d'une sous-variété abélienne de $A$, nous disposons d'une évaluation de sa hauteur. En effet, le résultat suivant affirme qu'à une constante près, qui ne dépend que de $(A, L)$, la hauteur de l'espace tangent $t_{B}$ d'une sous-variété abélienne $B$ de $A$ est du même ordre de grandeur que le logarithme de $\mathrm{h}^{0}(B, L)$, ou, ce qui revient au même puisque $L$ est ample, du degré de $B$ relativement à $L$, car on a la relation $\operatorname{deg}_{L} B=(\operatorname{dim} B) ! \mathrm{h}^{0}(B, L)$.

Lemme 4.8. Si $B$ est une sous-variété abélienne de $A$, la hauteur $h\left(t_{B}\right)$, relative à $(A, L)$, est encadrée de la manière suivante :

$$
\frac{1}{2} \log \mathrm{h}^{0}(B, L)-\frac{3}{2} \operatorname{dim} B \leq h\left(t_{B}\right) \leq h_{F}(A)+4 g \log \mathrm{h}^{0}(B, L)+\operatorname{codim} B .
$$

Ce lemme repose sur l'énoncé suivant qui est une partie de la proposition D.1 de [10].

Proposition 4.9. Si B est une sous-variété abélienne de A alors les hauteurs de Faltings vérifient

$$
h_{F}(B) \leq h_{F}(A)+(2 g-1) \log h^{0}(B, L)+\frac{(\operatorname{codim} B) \log (2 \pi)}{2}
$$

Démonstration. Nous reprenons la démonstration de Bost (ibid.). Considérons la sous-variété abélienne orthogonale $B^{\perp}$ de $B$ dans $A$ relativement à $L$. Le morphisme d'addition $B \times B^{\perp} \rightarrow A$ est une isogénie de degré $N \leq \mathrm{h}^{0}(B, L)^{2}$ (lemme 1.4 de [49]). L'isogénie duale $A \rightarrow B \times B^{\perp}$ est de degré $N^{2 g-1}$, et, d'après 22 , on a

$$
h_{F}(B)+h_{F}\left(B^{\perp}\right)=h_{F}\left(B \times B^{\perp}\right) \leq h_{F}(A)+\frac{1}{2} \log N^{2 g-1} .
$$

La minoration $h_{F}\left(B^{\perp}\right) \geq-\frac{(\operatorname{codim} B) \log (2 \pi)}{2}$ permet de conclure.

Démonstration du lemme 4.8. La proposition D.1 de 10] fournit l'encadrement

$$
h_{B, L}\left(t_{B}\right) \leq h_{A, L}\left(t_{B}\right) \leq h_{B, L}\left(t_{B}\right)+2 g \log \mathrm{h}^{0}(B, L)
$$


(avec une démonstration détaillée). Ici on a $h_{A, L}\left(t_{B}\right)=h\left(t_{B}\right)$ et la formule (23) appliquée à $B$ permet de calculer la hauteur $h_{B, L}\left(t_{B}\right)$. Les inégalités (26) découlent de cette estimation et de la proposition 4.9 .

4.4.2. Considérons maintenant des fibrés vectoriels hermitiens $\overline{\mathcal{E}}$ et $\overline{\mathcal{F}}$ sur Spec $\mathcal{O}_{k}$ et $f: \mathcal{E}_{k} \rightarrow \mathcal{F}_{k}$ une application $k$-linéaire. En chaque place $v$ de $k$, les espaces vectoriels $\mathcal{E} \otimes \overline{k_{v}}$ et $\mathcal{F} \otimes \overline{k_{v}}$ sur une clôture algébrique $\overline{k_{v}}$ de $k_{v}$ sont munis d'une métrique (lorsque $v$ est ultramétrique, voir le début du $\S 4.1$; désignons par $\|\cdot\|_{\overline{\mathcal{E}}, v}$ et $\|\cdot\|_{\overline{\mathcal{F}}, v}$ ces métriques ; alors, par définition, la hauteur $h(\overline{\mathcal{E}}, \overline{\mathcal{F}} ; f)$ (notée plus simplement $h(f))$ de $f$ relative à $\overline{\mathcal{E}}$ et $\overline{\mathcal{F}}$ est

$$
h(f):=\frac{1}{[k: \mathbf{Q}]} \sum_{v \text { place de } k} \log \sup _{x \in \mathcal{E}_{v} \backslash\{0\}}\left\{\frac{\|f(x)\|_{\overline{\mathcal{F}}_{, v}}}{\|x\|_{\overline{\mathcal{E}}, v}}\right\} .
$$

Par convention, si $f$ est l'application nulle, on pose $h(f)=-\infty$.

4.4.3. Dans le même esprit, étant donné une variété arithmétique $\mathcal{X}$ sur Spec $\mathcal{O}_{k}$, munie d'un fibré en droites hermitien $\overline{\mathcal{M}}$, la hauteur d'un point entier $\varepsilon_{x} \in \mathcal{X}\left(\mathcal{O}_{k}\right)$ est le degré d'Arakelov de $\overline{\varepsilon_{x}^{*} \mathcal{M}}$ :

$$
h \overline{\mathcal{M}}(x):=\widehat{\operatorname{deg}}_{\mathrm{n}} \overline{\varepsilon_{x}^{*} \mathcal{M}} .
$$

Cas d'un espace projectif. Supposons que $(\mathcal{X}, \overline{\mathcal{M}})$ est $\left(\mathbf{P}(\mathcal{E}), \overline{\mathcal{O}_{\mathbf{P}(\mathcal{E})}(1)}\right)$. Regardons comment évolue la hauteur $h_{\overline{\mathcal{O}_{\mathbf{P}(\mathcal{E})}(1)}}(x)$ lorsque $\overline{\mathcal{E}}$ varie en conservant le même rang. Pour cela, considérons $\overline{\mathcal{E}}, \overline{\mathcal{F}}$ des fibrés vectoriels hermitiens sur $\mathcal{O}_{k}$ et un isomorphisme $\phi: \mathcal{E}_{k} \rightarrow \mathcal{F}_{k}$. Soit $x \in \mathbf{P}\left(\mathcal{E}_{k}\right)(k)$ et $V_{x}$ l'hyperplan de $\mathcal{E}_{k}$ correspondant à $x$. Notons $\widetilde{\phi}: \mathcal{E}_{k} / V_{x} \rightarrow \mathcal{F}_{k} / \phi\left(V_{x}\right)$ l'application déduite de $\phi$ et $\phi(x) \in \mathbf{P}\left(\mathcal{F}_{k}\right)(k)$ le $k$-point de $\mathbf{P}\left(\mathcal{F}_{k}\right)$ correspondant à l'hyperplan $\phi\left(V_{x}\right)$ de $\mathcal{F}_{k}$. Alors on a

$$
h \frac{}{\mathcal{O}_{\mathbf{P}(\mathcal{E})}(1)}(x)=h \frac{}{\mathcal{O}_{\mathbf{P}(\mathcal{F})}(1)}(\phi(x))+h(\widetilde{\phi}) .
$$

En effet, il suffit de remarquer que l'on dispose d'isomorphismes isométriques

$$
\mathcal{E}_{k} / V_{x} \simeq x^{*} \mathcal{O}_{\mathbf{P}(\mathcal{E})}(1) \quad \text { et } \quad \mathcal{F}_{k} / \phi\left(V_{x}\right) \simeq \phi(x)^{*} \mathcal{O}_{\mathbf{P}(\mathcal{F})}(1)
$$

et d'appliquer la définition (28). Une conséquence est l'inégalité

$$
h \overline{\mathcal{O}_{\mathbf{P}(\mathcal{F})}(1)}(\phi(x)) \leq h \overline{\mathcal{O}_{\mathbf{P}(\mathcal{E})}(1)}(x)+h\left(\phi^{-1}\right) .
$$

Par exemple, si $\overline{\mathcal{E}}=\overline{\mathcal{O}_{k}^{N+1}}$ et

$$
\phi\left(\left(x_{0}, \ldots, x_{N}\right)\right)=\left(x_{0}, m x_{1}, \ldots, m x_{N}\right)
$$

(multiplication par un entier $m \neq 0)$, cela redonne l'inégalité classique :

$$
h \overline{\mathcal{O}_{\mathbf{P}^{N}}(1)}(m x) \leq h \overline{\mathcal{O}_{\mathbf{P}^{N}}(1)}(x)+\log m .
$$

D'autre part, toujours dans le cas $\overline{\mathcal{E}}=\overline{\mathcal{O}_{k}^{N+1}}$ et étant donné $x=\left(x_{0}: \cdots: x_{N}\right) \in \mathbf{P}^{N}(k)$, on montre que (voir par exemple [13], p. 49)

$$
h \frac{1}{\mathcal{O}_{\mathbf{P}^{N}}(1)}(x)=\frac{1}{[k: \mathbf{Q}]} \log \frac{\prod_{\sigma: k \hookrightarrow \mathbf{C}}\left(\sum_{i=0}^{N}\left|\sigma\left(x_{i}\right)\right|^{2}\right)^{1 / 2}}{\mathrm{~N}_{k \mid \mathbf{Q}}\left(x_{0} \mathcal{O}_{k}+\cdots+x_{N} \mathcal{O}_{k}\right)}
$$

où, si $I$ est un idéal fractionnaire de $\mathcal{O}_{k}$, le nombre rationnel $\mathrm{N}_{k \mid \mathbf{Q}}(I)$ est la norme de $I$. Cette formule permet de comparer $h_{\overline{\mathcal{O}_{\mathbf{P}^{N}}}(1)}(x)$ avec la hauteur logarithmique absolue de Weil de $x$ :

$$
h_{\mathrm{Weil}}(x) \leq h_{\overline{\mathcal{O}_{\mathrm{P} N}}(1)}(x) \leq h_{\mathrm{Weil}}(x)+\frac{1}{2} \log (1+N) .
$$

Enfin, notons que la hauteur du point « origine » $1 \oplus 0$ de $\mathcal{O}_{k} \oplus \mathcal{E}$, relative à $\overline{\mathcal{O}_{\mathbf{P}\left(\mathcal{O}_{k} \oplus \mathcal{E}\right)}(1)}$, est nulle.

Cas d'une variété abélienne. Supposons maintenant que $(\mathcal{X}, \overline{\mathcal{M}})=(\mathcal{A}, \overline{\mathcal{L}})$ est un modèle de Moret-Bailly sur $\operatorname{Spec} \mathcal{O}_{K}$ d'une variété abélienne polarisée $(A, L)$ définie sur $k$ (le corps $K$ est une extension finie de $k$ ). Le schéma en groupes $\mathcal{A}$ n'est en général qu'une partie ouverte du modèle de Néron de $A_{K}$. Aussi un point $x \in A(K)$ ne se prolonge pas nécessairement en $\varepsilon_{x} \in \mathcal{A}\left(\mathcal{O}_{K}\right)$. Toutefois la construction de $\mathcal{A}$ (p. 58 de [9]) permet de voir que cela devient possible si l'on remplace $K$ par une extension finie (qui dépend de $x$ ). C'est pourquoi, lorsque seul un nombre fini 
de points $x$ interviennent (comme dans la définition 4.3), l'on peut toujours supposer dès le départ que $x \in A(K)$ se prolonge en $\varepsilon_{x} \in \mathcal{A}\left(\mathcal{O}_{K}\right)$.

Théorème 4.10 (Moret-Bailly, chapitre III de [51). Avec les notations ci-dessus, la hauteur $h_{\overline{\mathcal{L}}}(x)=\widehat{\operatorname{deg}}_{\mathrm{n}} \overline{\varepsilon_{x}^{*} \mathcal{L}}$ d'un point $x$ de $A(K)$ coïncide avec la hauteur de Néron-Tate de $x$ relative à la polarisation $L$.

4.5. Inégalités de pentes. Après avoir introduit dans les paragraphes qui précèdent les notions de degré et pente d'Arakelov d'une part et de hauteur d'un morphisme entre fibrés vectoriels hermitiens d'autre part, nous présentons ici quelques inégalités « fondamentales » qui relient ces objets entre eux. Ces inégalités — dites inégalités de pentes — se révèleront particulièrement importantes dans la suite.

Soit $\overline{\mathcal{E}}$ et $\overline{\mathcal{F}}$ des fibrés vectoriels hermitiens sur $\operatorname{Spec} \mathcal{O}_{k}$. La définition même de la notion de pente maximale entraîne $\widehat{\operatorname{deg}}_{n} \overline{\mathcal{E}} \leq \operatorname{rg}(\mathcal{E}) \widehat{\mu}_{\max }(\overline{\mathcal{E}})$ et, si $\mathcal{F}$ est de rang 1 , on a

$$
\widehat{\mu}_{\text {max }}(\overline{\mathcal{E}} \otimes \overline{\mathcal{F}})=\widehat{\mu}_{\text {max }}(\overline{\mathcal{E}})+\widehat{\operatorname{deg}}_{\mathrm{n}} \overline{\mathcal{F}} .
$$

Cette égalité s'obtient par exemple en considérant un sous-fibré $\overline{\mathcal{E}^{\prime}}$ de $\overline{\mathcal{E}} \otimes \overline{\mathcal{F}}$ que l'on tensorise par le dual $\overline{\mathcal{F}^{v}}$ de $\overline{\mathcal{F}}$ pour le voir comme un sous-fibré de $\overline{\mathcal{E}}$ et auquel on applique alors la définition de pente maximale $: \widehat{\mu}\left(\overline{\mathcal{E}^{\prime}} \otimes \overline{\mathcal{F}^{v}}\right) \leq \widehat{\mu}_{\max }(\overline{\mathcal{E}})$. On conclut en utilisant l'égalité $\widehat{\mu}\left(\overline{\mathcal{E}^{\prime}} \otimes \overline{\mathcal{F} v}\right)=\widehat{\mu}\left(\overline{\mathcal{E}^{\prime}}\right)-\widehat{\operatorname{deg}}_{\mathrm{n}} \overline{\mathcal{F}}$.

Soit $\ell$ un entier $\geq 1$. Alors la pente maximale du fibré hermitien $S^{\ell}(\mathcal{E})$ (défini au $\S$ 4.1) est majorée de la manière suivante :

$$
\widehat{\mu}_{\max }\left(S^{\ell}(\overline{\mathcal{E}})\right) \leq \ell\left(\widehat{\mu}_{\max }(\overline{\mathcal{E}})+2 \operatorname{rg}(\mathcal{E}) \log \operatorname{rg}(\mathcal{E})\right)
$$

(inégalité (4.6), p. 196, de 12]). Ce résultat ne découle pas immédiatement des définitions. Une preuve détaillée est donnée dans l'appendice de [33].

Considérons maintenant une application $k$-linéaire $\varphi$ entre les espaces vectoriels $\mathcal{E}_{k}$ et $\mathcal{F}_{k}$. Dans le lemme suivant, nous présentons deux exemples caractéristiques d'inégalités de pentes, extraits de la proposition 4.3 de [10].

\section{Lemme 4.11.}

(1) Si $\varphi: \mathcal{E}_{k} \rightarrow \mathcal{F}_{k}$ est injective alors $\widehat{\mu}_{\max }(\overline{\mathcal{E}}) \leq \widehat{\mu}_{\max }(\overline{\mathcal{F}})+h(\varphi)$.

(2) Si $\varphi: \mathcal{E}_{k} \rightarrow \mathcal{F}_{k}$ est surjective alors

$$
\widehat{\mu}_{\max }(\overline{\mathcal{F}}) \leq \widehat{\operatorname{deg}}_{\mathrm{n}} \overline{\mathcal{F}}+(\operatorname{rg}(\mathcal{F})-1)\left(\widehat{\mu}_{\max }\left(\overline{\mathcal{E}^{v}}\right)+h(\varphi)\right) .
$$

Démonstration. (1) Soit $\overline{\mathcal{E}^{\prime}}$ un sous-fibré vectoriel hermitien de $\overline{\mathcal{E}}$. D'après l'hypothèse d'injectivité, l'application restreinte $\widetilde{\varphi}:=\varphi_{\mid \mathcal{E}_{k}^{\prime}}^{\left.\mid \mathcal{E}_{k}^{\prime}\right)}: \mathcal{E}_{k}^{\prime} \rightarrow \varphi\left(\mathcal{E}_{k}^{\prime}\right)$ est un isomorphisme d'espaces vectoriels. En particulier la norme d'opérateur de l'application déterminant de $\widetilde{\varphi}$ :

$$
\operatorname{det} \widetilde{\varphi}: \operatorname{det} \mathcal{E}_{k}^{\prime} \rightarrow \operatorname{det} \varphi\left(\mathcal{E}_{k}^{\prime}\right)
$$

en une place complexe $\sigma$ de $k$ est donnée par

$$
\|\operatorname{det} \widetilde{\varphi}\|_{\sigma}=\frac{\|\widetilde{\varphi}(x)\|_{\operatorname{det}} \overline{\varphi\left(\mathcal{E}^{\prime}\right)}, \sigma}{\|x\|_{\operatorname{det} \overline{\mathcal{E}}, \sigma}} \quad \text { pour tout } x \in \operatorname{det} \mathcal{E}_{\sigma}^{\prime} \backslash\{0\} .
$$

Les définitions du degré d'Arakelov et de la hauteur d'un morphisme entraînent

$$
\widehat{\operatorname{deg}}_{\mathrm{n}} \overline{\mathcal{E}^{\prime}}=\widehat{\operatorname{deg}}_{\mathrm{n}} \overline{\varphi\left(\mathcal{E}^{\prime}\right)}+h\left(\operatorname{det} \overline{\mathcal{E}^{\prime}}, \operatorname{det} \overline{\varphi\left(\mathcal{E}^{\prime}\right)} ; \operatorname{det} \widetilde{\varphi}\right) .
$$

En vertu de l'inégalité d'Hadamard, on a

$$
h\left(\operatorname{det} \overline{\mathcal{E}^{\prime}}, \operatorname{det} \overline{\varphi\left(\mathcal{E}^{\prime}\right)} ; \operatorname{det} \widetilde{\varphi}\right) \leq\left(\operatorname{dim} \mathcal{E}_{k}^{\prime}\right) h\left(\overline{\mathcal{E}^{\prime}}, \overline{\varphi\left(\mathcal{E}^{\prime}\right)} ; \widetilde{\varphi}\right)
$$

et cette dernière quantité est elle-même inférieure à $\left(\operatorname{dim} \mathcal{E}_{k}^{\prime}\right) h(\bar{E}, \bar{F} ; \varphi)$. Nous obtenons ainsi la majoration

$$
\widehat{\mu}\left(\overline{\mathcal{E}^{\prime}}\right) \leq \widehat{\mu}_{\max }(\overline{\mathcal{F}})+h(\bar{E}, \bar{F} ; \varphi)
$$

puis l'inégalité voulue en prenant la borne supérieure du membre de gauche.

(2) L'hypothèse de surjectivité de $\varphi$ signifie que l'application duale ${ }^{\mathrm{t}} \varphi: \mathcal{F}_{k}^{\vee} \rightarrow \mathcal{E}_{k}^{\vee}$ est injective et l'inégalité de pentes précédente montre que

$$
\widehat{\mu}_{\max }\left(\overline{\mathcal{F}^{v}}\right) \leq \widehat{\mu}_{\max }\left(\overline{\mathcal{E}^{v}}\right)+h\left(\overline{\mathcal{F}^{v}}, \overline{\mathcal{E}^{v}} ;{ }^{\mathrm{t}} \varphi\right) .
$$


La définition des normes duales assure l'égalité des hauteurs

$$
h\left(\overline{\mathcal{F}^{v}}, \overline{\mathcal{E}^{v}} ;{ }^{\mathrm{t}} \varphi\right)=h(\overline{\mathcal{E}}, \overline{\mathcal{F}} ; \varphi) .
$$

De plus, pour tout sous-fibré vectoriel (non nul) $\mathcal{F}^{\prime}$ de $\mathcal{F}$, la surjection canonique $\mathcal{F} \rightarrow \mathcal{F} / \mathcal{F}^{\prime}$ induit une injection sur les espaces duaux, qui est de norme d'opérateur $\leq 1$ en toute place de $k$. En utilisant à nouveau la première inégalité de pentes, nous obtenons alors la majoration

$$
\widehat{\mu}\left(\overline{\left(\mathcal{F} / \mathcal{F}^{\prime}\right)^{v}}\right) \leq \widehat{\mu}_{\max }(\overline{\mathcal{F} v})
$$

En multipliant cette inégalité par $\operatorname{rg}(\mathcal{F})-\operatorname{rg}\left(\mathcal{F}^{\prime}\right)$, nous obtenons une majoration du degré d'Arakelov normalisé de $\overline{\mathcal{F}^{\prime}}$ qui, si nous redivisons $\operatorname{par} \operatorname{rg}\left(\mathcal{F}^{\prime}\right)$, donne

$$
\begin{aligned}
\widehat{\mu}\left(\overline{\mathcal{F}^{\prime}}\right) & \leq \frac{\widehat{\operatorname{deg}}_{\mathrm{n}} \overline{\mathcal{F}}}{\operatorname{rg}\left(\mathcal{F}^{\prime}\right)}+\left(\frac{\operatorname{rg}(\mathcal{F})}{\operatorname{rg}\left(\mathcal{F}^{\prime}\right)}-1\right) \widehat{\mu}_{\max }\left(\overline{\mathcal{F}^{v}}\right) \\
& \leq \widehat{\operatorname{deg}}_{\mathrm{n}} \overline{\mathcal{F}}+(\operatorname{rg}(\mathcal{F})-1) \widehat{\mu}_{\text {max }}(\overline{\mathcal{F} v})
\end{aligned}
$$

(la différence entre les deux membres de droite vaut

$$
\operatorname{rg}(\mathcal{F}) \cdot\left(\frac{1}{\operatorname{rg}\left(\mathcal{F}^{\prime}\right)}-1\right) \cdot\left(\widehat{\mu}(\overline{\mathcal{F}})+\widehat{\mu}_{\max }(\overline{\mathcal{F} v})\right)
$$

et la dernière parenthèse est positive car $\widehat{\mu}(\overline{\mathcal{F}})=-\widehat{\mu}(\overline{\mathcal{F}}))$. On conclut avec (34).

Notons que ces inégalités restent vraies si $\mathcal{E}, \mathcal{F}$ ou $\varphi$ est nul grâce aux conventions choisies pour les degré, pente ou hauteur de morphisme dans ce cas, et à condition d'utiliser la règle de calcul classique en théorie de l'intégration : $0 \cdot \infty=0$ (ce que nous ferons implicitement dans toute la suite).

Méthode des pentes. L'ossature de la démonstration du théorème 3.1 repose sur une généralisation de la première inégalité de pentes du lemme 4.11. Pour assurer la cohérence des notations avec celles employées durant la preuve, considérons une extension finie $K \mid k$ et $\varphi: E \rightarrow F$ une application $K$-linéaire entre deux $K$-espaces vectoriels $E$ et $F$. Soit

$$
\{0\}=: F_{N} \subseteq F_{N-1} \subseteq \cdots \subseteq F_{0}:=F
$$

une filtration de $F$ par des $K$-espaces vectoriels $\left(N \in \mathbf{N}^{*}\right)$. Posons, pour $1 \leq i \leq N, G_{i}:=F_{i-1} / F_{i}$ et supposons que $E$ et les $G_{i}$ proviennent de fibrés hermitiens $\overline{\mathcal{E}}$ et $\overline{\mathcal{G}}_{i}$ (respectivement) sur $\operatorname{Spec} \mathcal{O}_{K}$. Notons $E_{i}:=\varphi^{-1}\left(F_{i-1}\right)$ pour $1 \leq i \leq N+1$ et $\varphi_{i}: E_{i} \rightarrow G_{i}$ l'application composée de la restriction $\varphi_{\mid E_{i}}$ et de la projection $F_{i-1} \rightarrow G_{i}$. Le $\mathcal{O}_{K}$-module saturé $\mathcal{E}_{i}:=E_{i} \cap \mathcal{E}$ hérite de la structure fibré vectoriel hermitien $\overline{\mathcal{E}}_{i}$ induite par $\overline{\mathcal{E}}$.

Lemme 4.12. Si $\varphi$ est injective alors

$$
\widehat{\operatorname{deg}}_{\mathrm{n}} \overline{\mathcal{E}} \leq \sum_{i=1}^{N} \operatorname{dim}\left(E_{i} / E_{i+1}\right)\left(\widehat{\mu}_{\max }\left(\overline{\mathcal{G}}_{i}\right)+h\left(\overline{\mathcal{E}}_{i}, \overline{\mathcal{G}}_{i} ; \varphi_{i}\right)\right) .
$$

Démonstration. L'hypothèse sur $\varphi$ implique que $\varphi_{i}$ se factorise par l'injection $\widetilde{\varphi}_{i}: E_{i} / E_{i+1} \hookrightarrow G_{i}$. La première inégalité de pentes du lemme 4.11 entraîne alors la majoration

$$
\widehat{\operatorname{deg}}_{\mathrm{n}} \overline{\mathcal{E}_{i} / \mathcal{E}_{i+1}} \leq \operatorname{dim}\left(E_{i} / E_{i+1}\right)\left(\widehat{\mu}_{\max }\left(\overline{\mathcal{G}}_{i}\right)+h\left(\overline{\mathcal{E}_{i} / \mathcal{E}_{i+1}}, \overline{\mathcal{G}}_{i} ; \widetilde{\varphi}_{i}\right)\right) .
$$

On conclut en sommant ces inégalités de $i=1$ à $N$ et en remarquant d'une part que

$$
h\left(\overline{\mathcal{\mathcal { E }}_{i} / \mathcal{E}_{i+1}}, \overline{\mathcal{G}}_{i} ; \widetilde{\varphi}_{i}\right) \leq h\left(\overline{\mathcal{E}}_{i}, \overline{\mathcal{G}}_{i} ; \varphi_{i}\right)
$$

et d'autre part que

$$
\sum_{i=1}^{N} \widehat{\operatorname{deg}}_{\mathrm{n}} \overline{\mathcal{E}_{i} / \mathcal{E}_{i+1}}=\widehat{\operatorname{deg}}_{\mathrm{n}} \overline{\mathcal{E}}=(\operatorname{dim} E) \widehat{\mu}(\overline{\mathcal{E}})
$$

(voir la proposition 4.6 de [12]).

La méthode des pentes s'articule autour de cette inégalité. Selon le problème envisagé, on choisit les données $\overline{\mathcal{E}},\left(\overline{\mathcal{G}}_{i}\right)_{i},\left(\varphi_{i}\right)_{i}$ puis l'on évalue chacune des quantités qui interviennent dans l'inégalité (35). L'information qui en résulte conduit ou non à la conclusion (voir $\S 5.1$ ). 
4.6. Comparaisons de normes. L'objectif de ce paragraphe est de comparer la norme du supremum et la norme $\mathrm{L}^{2}$ d'une section d'un fibré vectoriel hermitien sur une variété complexe (compacte). L'archétype d'une telle inégalité se trouve au § 5.2.3 de [31], sous la dénomination « inégalité de Gromov ». Soit $\pi: X \rightarrow \mathbf{C}$ une variété analytique complexe compacte et $\bar{L}=(L,\|\cdot\|)$ un fibré en droites hermitien ample sur $X$. Soit $\mathrm{d} \mu$ une mesure de probabilité sur $X(\mathbf{C})$. L'espace des sections globales $\mathrm{H}^{0}(X, L)$ peut être muni d'une structure d'espace hermitien en considérant la norme :

$$
\forall s \in \mathrm{H}^{0}(X, L), \quad\|s\|_{2}:=\left(\int_{X(\mathbf{C})}\|s(x)\|_{\frac{2}{L_{x}}} \mathrm{~d} \mu(x)\right)^{1 / 2}
$$

ainsi que d'une structure d'espace de Banach au moyen de la norme du supremum :

$$
\forall s \in \mathrm{H}^{0}(X, L), \quad\|s\|_{\infty}:=\sup _{x \in X(\mathbf{C})}\|s(x)\|_{\overline{L_{x}}} .
$$

La quantité qui nous intéresse ici est

$$
\Xi(X, \bar{L}, \mathrm{~d} \mu):=\log \sup \left\{\frac{\|s\|_{\infty}}{\|s\|_{2}} ; s \in \mathrm{H}^{0}(X, L) \backslash\{0\}\right\} .
$$

Ce nombre réel est positif. L'ensemble des triplets $(X, \bar{L}, \mathrm{~d} \mu)$ possède une loi interne qui à $\left(X_{1}, \bar{L}_{1}, \mathrm{~d} \mu_{1}\right)$ et $\left(X_{2}, \bar{L}_{2}, \mathrm{~d} \mu_{2}\right)$ associe $\left(X_{1} \times X_{2}, \bar{L}_{1} \otimes \bar{L}_{2}, \mathrm{~d} \mu_{1} \otimes \mathrm{d} \mu_{2}\right)$ où, dans cette écriture, $X_{1} \times X_{2}$ désigne la variété produit et $\mathrm{d} \mu_{1} \otimes \mathrm{d} \mu_{2}$ la mesure de probabilité produit. Quant au fibré en droites hermitien $\bar{L}_{1} \otimes \bar{L}_{2}$ sur $X_{1} \times X_{2}$, il se compose du produit externe $L_{1} \otimes L_{2}=p_{1}^{*} L_{1} \otimes p_{2}^{*} L_{2}$ ( $p_{i}$ projection de $X_{1} \times X_{2}$ sur $X_{i}$ pour $\left.i=1,2\right)$ et de la norme tensorielle sur les fibres $\left(L_{1} \otimes L_{2}\right)_{\left(x_{1}, x_{2}\right)}=$ $\left(L_{1}\right)_{x_{1}} \otimes\left(L_{2}\right)_{x_{2}}$. Cette loi interne est compatible avec $\Xi$ au sens suivant :

Proposition 4.13. Avec les notations ci-dessus, on a

$$
\Xi\left(X_{1} \times X_{2}, \bar{L}_{1} \otimes \bar{L}_{2}, \mathrm{~d} \mu_{1} \otimes \mathrm{d} \mu_{2}\right)=\Xi\left(X_{1}, \bar{L}_{1}, \mathrm{~d} \mu_{1}\right)+\Xi\left(X_{2}, \bar{L}_{2}, \mathrm{~d} \mu_{2}\right) .
$$

Démonstration. En vertu de la formule de Künneth, on a

$$
\mathrm{H}^{0}\left(X_{1} \times X_{2}, L_{1} \otimes L_{2}\right)=\mathrm{H}^{0}\left(X_{1}, L_{1}\right) \otimes \mathrm{H}^{0}\left(X_{2}, L_{2}\right) .
$$

Pour $i=1,2$, soit $s_{i} \in \mathrm{H}^{0}\left(X_{i}, L_{i}\right)$. La majoration

$$
\frac{\left\|s_{1}\right\|_{\infty}}{\left\|s_{1}\right\|_{2}} \times \frac{\left\|s_{2}\right\|_{\infty}}{\left\|s_{2}\right\|_{2}}=\frac{\left\|p_{1}^{*} s_{1} \otimes p_{2}^{*} s_{2}\right\|_{\infty}}{\left\|p_{1}^{*} s_{1} \otimes p_{2}^{*} s_{2}\right\|_{2}} \leq \exp \Xi\left(X_{1} \times X_{2}, \bar{L}_{1} \otimes \bar{L}_{2}, \mathrm{~d} \mu_{1} \otimes \mathrm{d} \mu_{2}\right)
$$

entraîne

$$
\Xi\left(X_{1}, \bar{L}_{1}, \mathrm{~d} \mu_{1}\right)+\Xi\left(X_{2}, \bar{L}_{2}, \mathrm{~d} \mu_{2}\right) \leq \Xi\left(X_{1} \times X_{2}, \bar{L}_{1} \otimes \bar{L}_{2}, \mathrm{~d} \mu_{1} \otimes \mathrm{d} \mu_{2}\right) .
$$

Inversement, si $s \in \mathrm{H}^{0}\left(X_{1} \times X_{2}, L_{1} \otimes L_{2}\right)$ alors la norme hermitienne de $s$ est caractérisée par la formule

$$
\|s\|_{2}=\inf \left\{\left\|\left(a_{1}, \ldots, a_{\ell}\right)\right\|_{2}\left\|\left(b_{1}, \ldots, b_{\ell}\right)\right\|_{2}^{w}\right\},
$$

où la borne inférieure porte sur toutes les représentations possibles de $s$ en une somme $\sum_{i=1}^{\ell} a_{i} \otimes b_{i}$, avec $a_{i} \in \mathrm{H}^{0}\left(X_{1}, L_{1}\right)$ et $b_{i} \in \mathrm{H}^{0}\left(X_{2}, L_{2}\right)$. Dans cette expression, le terme $\left\|\left(a_{1}, \ldots, a_{\ell}\right)\right\|_{2}$ vaut par définition $\left(\sum_{i=1}^{\ell}\left\|a_{i}\right\|_{2}^{2}\right)^{1 / 2}$ et

$$
\left\|\left(b_{1}, \ldots, b_{\ell}\right)\right\|_{2}^{w}:=\sup \left\{\left\|\sum_{i=1}^{\ell} \lambda_{i} b_{i}\right\|_{2} ; \lambda_{i} \in \mathbf{C} \text { et } \sum_{i=1}^{\ell}\left|\lambda_{i}\right|^{2} \leq 1\right\}
$$

(l'exposant $w$ pour weak). Soit $x_{1} \in X_{1}, x_{2} \in X_{2}$, e une base unitaire de la fibre $\left(L_{1}\right)_{x_{1}}$ et $a_{1}, \ldots, b_{\ell}$ comme ci-dessus représentant $s$. L'élément $a_{i}\left(x_{1}\right)$ s'écrit $\lambda_{i}\left(x_{1}\right)$ e pour un certain $\lambda_{i}\left(x_{1}\right) \in \mathbf{C}$ de module $\left\|a_{i}\left(x_{1}\right)\right\| \frac{}{\left(L_{1}\right)_{x_{1}}}$, plus petit donc que $\left\|a_{i}\right\|_{\infty}$. L'on en déduit l'existence de $\lambda_{i}^{\prime}\left(x_{1}\right) \in \mathbf{C}$ vérifiant $\sum_{i=1}^{\ell}\left|\lambda_{i}^{\prime}\left(x_{1}\right)\right|^{2} \leq 1$ et tels que

$$
s\left(x_{1}, x_{2}\right)=\left(\sum_{i=1}^{\ell}\left\|a_{i}\right\|_{\infty}^{2}\right)^{1 / 2} \mathrm{e} \otimes\left(\sum_{i=1}^{\ell} \lambda_{i}^{\prime}\left(x_{1}\right) b_{i}(y)\right) .
$$

De cette expression découle la majoration

$$
\left\|s\left(x_{1}, x_{2}\right)\right\| \leq\left(\sum_{i=1}^{\ell}\left\|a_{i}\right\|_{\infty}^{2}\right)^{1 / 2}\left\|\sum_{i=1}^{\ell} \lambda_{i}^{\prime}\left(x_{1}\right) b_{i}\right\|_{\infty} .
$$


Le passage aux normes $\mathrm{L}^{2}$ s'effectue avec les fonctions $\Xi$ de $\bar{L}_{1}$ et $\bar{L}_{2}$ et l'on majore ensuite le terme $\left\|\sum_{i=1}^{\ell} \lambda_{i}^{\prime}\left(x_{1}\right) b_{i}\right\|_{2}$ par la borne supérieure $\left\|\left(b_{1}, \ldots, b_{\ell}\right)\right\|_{2}^{w}$. Comme la norme de $s\left(x_{1}, x_{2}\right)$ ne dépend pas de la représentation choisie pour $s$, on obtient

$$
\left\|s\left(x_{1}, x_{2}\right)\right\| \leq \exp \left\{\Xi\left(X_{1}, \bar{L}_{1}, \mathrm{~d} \mu_{1}\right)+\Xi\left(X_{2}, \bar{L}_{2}, \mathrm{~d} \mu_{2}\right)\right\}\|s\|_{2}
$$

puis la majoration voulue pour $\sup \left\{\frac{\|s\|_{\infty}}{\|s\|_{2}} ; s \neq 0\right\}$.

La première évaluation de $\Xi(X, \bar{L}, \mathrm{~d} \mu)$ concerne le cas projectif. Soit $(E,\|\|$.$) un espace hermitien$ de dimension $N+1(N \in \mathbf{N})$. On a vu comment le faisceau canonique $\mathcal{O}_{\mathbf{P}(E)}(1)$ sur l'espace projectif $\mathbf{P}(E)$ était naturellement muni d'une structure de fibré hermitien grâce à la métrique de FubiniStudy, structure qui se transmet à $\mathcal{O}_{\mathbf{P}(E)}(m)$, pour tout $m$ entier $\geq 1$, par produit tensoriel. L'espace $\mathbf{P}(E)$ est une variété algébrique complexe compacte. Soit $\mathrm{d} \mu_{\mathrm{FS}}$ l'unique mesure de probabilité sur $\mathbf{P}(E)$ invariante sous l'action du groupe unitaire $\mathbf{U}_{N+1}:=\left\{P \in \mathrm{M}_{N+1}(\mathbf{C}) ; P^{-1}={ }^{t} \bar{P}\right\}$.

Proposition 4.14. Pour tout entier $m \geq 1$, on a

$$
\Xi\left(\mathbf{P}(E), \overline{\mathcal{O}_{\mathbf{P}(E)}(m)}, \mathrm{d} \mu_{\mathrm{FS}}\right)=\frac{1}{2} \log \left(\begin{array}{c}
N+m \\
N
\end{array}\right) .
$$

Démonstration. Soit $s \in \mathrm{H}^{0}\left(\mathbf{P}(E), \mathcal{O}_{\mathbf{P}(E)}(m)\right)$ et $P$ le polynôme homogène de degré $m$ de $\mathbf{C}\left[X_{0}, \ldots, X_{N}\right]$ qui représente $s$ dans une base orthonormée de $E$. Si l'on écrit

$$
P=\sum_{\substack{|\mathbf{i}|=m \\ \mathbf{i} \in \mathbf{N}^{N+1}}} p_{\mathbf{i}} X^{\mathbf{i}}
$$

et si $x=\left(x_{0}: \cdots: x_{N}\right) \in \mathbf{P}(E)(\mathbf{C})$ alors, grâce à l'inégalité de Cauchy-Schwarz et via la formule (18) exprimant $\|s\|_{\overline{\mathcal{O}_{\mathbf{P}(E)}(m)}}$, on a

$$
\begin{aligned}
\left|P\left(x_{0}, \ldots, x_{N}\right)\right| & =\left|\sum_{|\mathbf{i}|=m}\left(p_{\mathbf{i}} \sqrt{\frac{N ! \mathbf{i}}{(N+m) !}}\right) \times\left(x^{\mathbf{i}} \sqrt{\frac{(N+m) !}{N ! \mathbf{i} !}}\right)\right| \\
& \leq\|s\|_{\mathcal{O}_{\mathbf{P}(E)}(m)}\left(\sum_{|\mathbf{i}|=m} \frac{(N+m) !}{N ! \mathbf{i} !} x^{2 \mathbf{i}}\right)^{1 / 2} .
\end{aligned}
$$

En factorisant $\left(\begin{array}{c}N+m \\ N\end{array}\right)$ dans la dernière somme, on reconnaît le terme $\left(\left|x_{0}\right|^{2}+\cdots+\left|x_{N}\right|^{2}\right)^{m / 2}$ grâce à la formule du multinôme. La formule (17) entraîne alors

$$
\|s(x)\|_{\overline{\mathcal{O}}_{\mathbf{P}(E)}(m)} \leq\left(\begin{array}{c}
N+m \\
N
\end{array}\right)^{1 / 2}\|s\|_{\mathcal{O}_{\mathbf{P}(E)}(m)}
$$

et donc

$$
\Xi\left(\mathbf{P}(E), \overline{\mathcal{O}_{\mathbf{P}(E)}(m)}, \mathrm{d} \mu_{\mathrm{FS}}\right) \leq \frac{1}{2} \log \left(\begin{array}{c}
N+m \\
N
\end{array}\right) .
$$

Le cas d'égalité s'obtient en choisissant la section de polynôme associé $X_{0}^{m}$.

La fonction $\Xi$ restreinte aux variétés complexes est insuffisante pour l'usage que nous allons en faire plus tard. Aussi, avant d'obtenir d'autres évaluations pour les variétés abéliennes, commençons par définir un analogue arithmétique de $\Xi$.

Soit $\pi: \mathcal{X} \rightarrow \operatorname{Spec}\left(\mathcal{O}_{k}\right)$ une variété arithmétique projective (au sens de [9]) et $\overline{\mathcal{L}} \rightarrow \mathcal{X}$ un fibré en droites hermitien sur $\mathcal{X}$. Supposons que $\mathcal{L}_{k}$ est ample. Pour tout $\sigma: k \rightarrow \mathbf{C}$, considérons une mesure de probabilité $\mathrm{d} \mu_{\sigma}$ sur la variété complexe $\mathcal{X}_{\sigma}(\mathbf{C})$. Posons alors

$$
\Xi\left[\left(\mathcal{X}, \overline{\mathcal{L}},\left(\mathrm{d} \mu_{\sigma}\right)_{\sigma: k \hookrightarrow \mathbf{C}}\right)\right]:=\frac{1}{[k: \mathbf{Q}]} \sum_{\sigma: k \hookrightarrow \mathbf{C}} \Xi\left(\mathcal{X}_{\sigma}, \overline{\mathcal{L}}_{\sigma}, \mathrm{d} \mu_{\sigma}\right) .
$$

Comme le premier invariant $\Xi$, c'est un nombre réel positif qui est additif vis-à-vis du produit externe. De la proposition 4.14 découle immédiatement le 
Lemme 4.15. Soit $\overline{\mathcal{E}}$ un fibré vectoriel hermitien sur $\operatorname{Spec}\left(\mathcal{O}_{k}\right)$ de rang $N+1$. Alors, pour tout entier $m \geq 1$, on $a$

$$
\Xi\left[\left(\mathbf{P}(\mathcal{E}), \overline{\mathcal{O}_{\mathbf{P}(\mathcal{E})}(m)},\left(\mathrm{d} \mu_{\mathrm{FS}, \sigma}\right)_{\sigma: k \hookrightarrow \mathbf{C}}\right)\right]=\frac{1}{2} \log \left(\begin{array}{c}
N+m \\
N
\end{array}\right) .
$$

L'énoncé suivant pour les variétés abéliennes est plus difficile à établir.

Lemme 4.16. Soit $(A, L)$ une variété abélienne polarisée sur $k$ et $(\mathcal{A}, \overline{\mathcal{L}})$ un modèle de MoretBailly de $(A, L)$ sur $\operatorname{Spec} \mathcal{O}_{K}$, où $K$ est une extension finie de $k$. Étant donné un plongement complexe $\sigma$ de $K$, notons $\mathrm{d} \mu_{\sigma}$ la mesure de Haar normalisée sur $A_{\sigma}(\mathbf{C})$. Alors, pour tout entier $m \geq 1$, on $a$

$$
\Xi\left[\left(\mathcal{A}, \overline{\mathcal{L}^{\otimes m}},\left(\mathrm{~d} \mu_{\sigma}\right)_{\sigma: K \hookrightarrow \mathbf{C}}\right)\right] \leq \mathrm{c}_{0}(g) \max \left\{1, \log ^{+}\left(h_{F}(A)\right), \log \mathrm{h}^{0}(A, L), \log m\right\}
$$

où $\mathrm{c}_{0}(g)$ est une constante qui ne dépend que de la dimension de la variété abélienne. De plus, si la polarisation $L$ est principale, l'on peut prendre $\mathrm{c}_{0}(g)=4 g^{4}$.

La preuve de ce lemme repose sur une version effective du lemme matriciel de Masser, énoncé au $\S 3$, p. 115, de 47], ainsi que sur des estimations autour de fonctions thêta. La constante a été calculée par Graftieaux [33] (proposition 2.11). Nous reviendrons en détail sur ces techniques au prochain paragraphe.

Les estimations (38) et (39) se révèleront particulièrement précieuses lorsque nous serons amenés aux $\S \S 5.9$ et 5.10 à donner une majoration de la partie analytique des hauteurs des applications linéaires $\varphi_{i}$ construites au cours de la preuve du théorème 3.1. Il s'avère que la quantité (37) apparaît de manière récurrente dans les estimations analytiques nécessaires à l'évaluation de pentes maximales de fibrés vectoriels hermitiens. La raison en est qu'il est souvent plus simple de travailler au cours de la preuve avec la norme du supremum qu'avec la norme $\mathrm{L}^{2}$ originale. Par exemple, Bost a décrit au $\S 5.3 .4$ de [10] un procédé pour obtenir assez simplement une estimation de la pente maximale $\widehat{\mu}_{\max }\left(\overline{\mathcal{V}^{v}}\right)$ du dual du sous-fibré $\overline{\mathcal{V}}$ de $\overline{t_{\mathcal{A}}}$ à partir de « l'application de Shimura »:

$$
\Sigma: \mathrm{H}^{0}\left(\mathcal{A}, \mathcal{L}^{\otimes 3}\right)^{\otimes 2} \rightarrow t_{\mathcal{A}}^{\vee}, \quad \Sigma\left(s_{1} \otimes s_{2}\right)=\varepsilon^{*}\left(s_{2}^{\otimes 2} \otimes \mathrm{d}\left(s_{1} / s_{2}\right)\right)
$$

où $\varepsilon: \operatorname{Spec} \mathcal{O}_{K} \rightarrow \mathcal{A}$ est la section nulle de $\mathcal{A}$. L'application $\Sigma_{K}$ entre les $K$-espaces vectoriels correspondants est surjective. Grâce à la première inégalité de pentes du lemme 4.11 et à la formule de dualité $h\left({ }^{\mathrm{t}} \Sigma\right)=h(\Sigma)$, nous avons alors

$$
\widehat{\mu}_{\max }\left(\overline{t_{\mathcal{A}}}\right) \leq h_{F}(A)-\frac{1}{2} \log \left(\frac{3^{g} \mathrm{~h}^{0}(A, L)}{(2 \pi)^{g}}\right)+h(\Sigma) .
$$

Puis, si nous appliquons maintenant l'inégalité de pentes (33) au morphisme surjectif $t_{\mathcal{A}}^{\vee} \rightarrow \mathcal{V}^{\vee}($ de hauteur $\leq 0$ ), nous obtenons

$$
\begin{aligned}
\widehat{\mu}_{\text {max }}\left(\overline{\mathcal{V}^{v}}\right) \leq & \widehat{\operatorname{deg}}_{\mathrm{n}} \overline{\mathcal{V}^{v}}+(\operatorname{dim} \mathcal{V}-1) \widehat{\mu}_{\text {max }}\left(\overline{t_{\mathcal{A}}}\right) \\
\leq & \widehat{\operatorname{deg}}_{\mathrm{n}} \overline{\mathcal{V}^{v}}+(\operatorname{dim} \mathcal{V}-1)\left(h_{F}(A)-\frac{1}{2} \log \left(\frac{3^{g} \mathrm{~h}^{0}(A, L)}{(2 \pi)^{g}}\right)\right) \\
& +(\operatorname{dim} \mathcal{V}-1) h(\Sigma)
\end{aligned}
$$

Or, comme on le voit directement à partir des définitions (voir la preuve de la proposition 2.14 de [33]), on a

$$
h(\Sigma) \leq \log (6 g)+2 \Xi\left[\left(\mathcal{A}, \overline{\mathcal{L}^{\otimes 3}},\left(\mathrm{~d} \mu_{\sigma}\right)_{\sigma: K \hookrightarrow \mathbf{C}}\right)\right] .
$$

De la sorte, l'évaluation des pentes maximales $\widehat{\mu}_{\max }\left(\overline{t_{\mathcal{A}}}\right)$ et $\widehat{\mu}_{\max }\left(\overline{\mathcal{V}^{v}}\right)$ revient bien à estimer la quantité $\Xi\left[\left(\mathcal{A}, \overline{\mathcal{L}}{ }^{\otimes 3},\left(\mathrm{~d} \mu_{\sigma}\right)_{\sigma: K \hookrightarrow \mathbf{C}}\right)\right]$. Un calcul élémentaire et le lemme 4.16 fournissent alors les majorations

$$
\widehat{\mu}_{\max }\left(\overline{t_{\mathcal{A}}}\right) \leq \mathrm{c}_{1}(g) \max \left\{1, h_{F}(A), \log \mathrm{h}^{0}(A, L)\right\}
$$

et

$$
\widehat{\mu}_{\max }\left(\overline{\mathcal{V}^{\mathrm{v}}}\right) \leq \mathrm{c}_{2}(g) \max \left\{1, h_{F}(A), \log \mathrm{h}^{0}(A, L), h(\mathcal{V})\right\}
$$

avec, en outre, $\mathrm{c}_{1}(g)=14 g^{4}\left(\right.$ resp. $\left.\mathrm{c}_{2}(g)=14 g^{5}\right)$ si la polarisation $L$ est principale. 
4.7. Un lemme matriciel. Soit $(A, L)$ une variété abélienne polarisée sur un corps de nombres $k$, comme précédemment. L'objectif de ce paragraphe est d'obtenir une majoration de la distance d'un vecteur $z \in t_{A_{\sigma}}(\mathbf{C})$ de l'espace tangent de $A_{\sigma}(\mathbf{C})$ au réseau des périodes de cette variété abélienne. Étant donné un plongement complexe $\sigma: k \hookrightarrow \mathbf{C}$ et $x \in A(k)$, notons $r_{\sigma}(x)$ la norme minimale (relative à $t_{A_{\sigma}(\mathbf{C})}$ ) d'un logarithme non nul de $\sigma(x) \in A_{\sigma}(\mathbf{C})$ :

$$
r_{\sigma}(x):=\inf \left\{\|z\|_{\sigma} ; z \in t_{A_{\sigma}}(\mathbf{C}) \backslash\{0\} \text { et } \exp _{A_{\sigma}(\mathbf{C})}(z)=\sigma(x)\right\}
$$

La norme $\|\cdot\|_{\sigma}$ est celle définie par la formule (1) de l'introduction, p. [1. La borne supérieure des $r_{\sigma}(x)$ pour $x$ variant dans $A_{\sigma}(\mathbf{C})$ (et non seulement $A(k)$ ) est le rayon de recouvrement du réseau des périodes de $A_{\sigma}(\mathbf{C})$.

Proposition 4.17. Soit $(A, L)$ une variété abélienne principalement polarisée ( $L$ est un fibré en droites ample et symétrique sur $A)$ sur un corps de nombres $k$. Alors, pour tout élément $x \in A(k)$, on a

$$
\frac{1}{[k: \mathbf{Q}]} \sum_{\sigma: k \hookrightarrow \mathbf{C}} \log ^{+} r_{\sigma}(x) \leq g \log ^{+}\left\{h_{F}(A)\right\}+5 g^{2} \log (g+1) .
$$

\section{Remarques 4.18.}

(1) Lorsque $L$ n'est pas principal, nous disposons encore d'une borne de la forme

$$
c(g) \max \left\{1, \log ^{+}\left\{h_{F}(A)\right\}, \log \mathrm{h}^{0}(A, L)\right\}
$$

avec $c(g)$ une constante qui ne dépend que de $g$.

(2) Au cours de la preuve du théorème 3.1 (p. 50), nous utiliserons plutôt la majoration simplifiée $5 g^{3} \max \left\{1, \mathrm{~h}_{F}\right\}$, qui est obtenue en remarquant que $\log ^{+}\left\{h_{F}(A)\right\} \leq \log (n)+$ $\log ^{+} \mathrm{h}_{F}$ et $5 g^{2} x \geq \log (x)+\log (g)+5 g \log (g+1), \forall x \geq 1$.

Pour démontrer ce résultat, nous allons procéder de la manière suivante. Soit $\sigma: k \hookrightarrow \mathbf{C}$ un plongement complexe de $k$. Le théorème des minima successifs de Minkowski fournit une famille libre (et génératrice sur $\mathbf{Q}$ ) d'éléments de $\Omega_{A_{\sigma}}(\mathbf{C})$ de normes « petites ». La quantité $r_{\sigma}(x)$ est alors majorée par $g$ fois le norme du plus grand des vecteurs de cette famille. Pour évaluer celle-ci, il revient au même de minorer la norme de la plus petite période non nulle de $A_{\sigma}(\mathbf{C})$. Un tel résultat porte le nom de lemme matriciel, suivant la terminologie introduite par Masser. Des énoncés de ce type ont été formulés par Masser [47] et Bost [10]. Graftieaux a obtenu une variante totalement effective lorsque la variété abélienne est principalement polarisée (lemme 2.12 de [33], p. 99) et David \& Philippon ont démontré un énoncé voisin de la proposition 4.17 avec une hauteur thêta à la place de la hauteurs de Faltings de $A$ [24]. Dans ce qui suit, nous nous sommes fortement inspiré de ces derniers travaux en apportant quelques modifications techniques qui améliorent les constantes. Avant de démontrer la proposition 4.17, commençons par rappeler les énoncés précis des résultats mentionnés ci-dessus.

La formulation suivante du théorème des minima successifs de Minkowski est extraite du livre de Martinet [44], corollaire 2.6.9 et remarque 2.7.5.

Lemme 4.19. Soit $(\mathrm{E},\|\|$.$) un espace euclidien de dimension \mathrm{n}$ et $\Lambda$ un réseau de $\mathrm{E}$. Notons $\operatorname{covol}(\Lambda)$ le volume de l'espace compact $\mathrm{E} / \Lambda$. Il existe une constante $\mathfrak{c}_{\mathrm{n}}$, ne dépendant que de $\mathrm{n}$, et des éléments $\lambda_{1}, \ldots, \lambda_{\mathrm{n}}$ de $\Lambda$ qui sont linéairement indépendants tels que

De plus, on a $\mathfrak{c}_{\mathrm{n}} \leq 1+\mathrm{n} / 4$.

$$
\left\|\lambda_{1}\right\| \cdots\left\|\lambda_{\mathrm{n}}\right\| \leq \mathfrak{c}_{\mathrm{n}}^{\mathrm{n} / 2} \operatorname{covol}(\Lambda)
$$

Évoquons également quelques aspects de la théorie de la réduction des matrices symétriques. Une présentation plus complète de cette théorie se trouve dans le livre de Igusa [39] (en particulier le $\S 4 \mathrm{du}$ chapitre V) ainsi que, sous un angle plus quantitatif, dans l'appendice de l'article de David \& Philippon 24]. Soit $g \in \mathbf{N} \backslash\{0\}$. Notons $\mathrm{S}_{g}$ l'espace des matrices symétriques réelles, $\mathrm{S}_{g}^{+}$ le sous-ensemble des matrices définies positives et $\mathfrak{S}_{g}$ l'espace de Siegel $\mathrm{S}_{g}+i \mathrm{~S}_{g}^{+}$.

Définition 4.20. On dit qu'une matrice $y=\left(y_{i, j}\right) \in \mathrm{S}_{g}^{+}$est réduite au sens de Minkowski si

(i) pour tout $\xi=\left(\xi_{1}, \ldots, \xi_{g}\right) \in \mathbf{Z}^{g}$ et tout entier $i \in\{1, \ldots, g\}$ tels que $\xi_{i}, \ldots, \xi_{g}$ sont premiers entre eux, on a ${ }^{\mathrm{t}} \xi y \xi \geq y_{i, i}$ 
(ii) pour tout $i \in\{1, \ldots, g-1\}, y_{i, i+1} \geq 0$.

Ajoutons que dans la première condition l'on peut remplacer «premiers entre eux » par « non tous nuls ». De plus, si $e_{1}, \ldots, e_{g}$ désigne les vecteurs de la base canonique de $\mathbf{Z}^{g}$, on montre en choisissant successivement $\xi=e_{i}$ et $\xi=e_{i} \pm e_{j}$ que les coefficients de $y$ vérifient $0<y_{1,1} \leq \cdots \leq y_{g, g}$ et $\left|y_{i, j}\right| \leq y_{i, i} / 2$ pour $i, j \in\{1, \ldots, g\}$. Il nous faut également quelques informations quantitatives supplémentaires sur ces matrices.

Lemme 4.21. Soit $y \in \mathrm{S}_{g}^{+}$une matrice réduite au sens de Minkowski et $y_{1}, \ldots, y_{g}$ ses coefficients sur la diagonale. Alors on a les propriétés suivantes.

(a) $y_{1} \cdots y_{g} \leq \mathfrak{c}_{g}^{g} \operatorname{det}(y)$,

(b) pour tout $\xi=\left(\xi_{1}, \ldots, \xi_{g}\right) \in \mathbf{R}^{g}$, on a

$$
\sum_{i=1}^{g} y_{i} \xi_{i}^{2} \leq c_{9}{ }^{\mathrm{t}} \xi y \xi \quad \text { avec } c_{9}=\left(\frac{g+1}{2}\right)^{g-1} \mathfrak{c}_{g}^{g} .
$$

Démonstration. La première inégalité résulte du théorème de Minkowski (lemme 4.19) appliqué au réseau $y^{1 / 2} \mathbf{Z}^{g}$ de l'espace euclidien usuel $\mathbf{R}^{g}$. Le covolume de ce réseau est $\operatorname{det}\left(y^{1 / 2}\right)=\sqrt{\operatorname{det}(y)}$. Il existe $b_{1}, \ldots, b_{g} \in \mathbf{Z}^{g}$ qui forment une base de $\mathbf{R}^{g}$ tels que $\prod_{i=1}^{g}\left\|y^{1 / 2} b_{i}\right\|^{2} \leq \mathfrak{c}_{g}^{g} \operatorname{det}(y)$. Notons $\left(b_{j, m}\right)_{1 \leq m \leq g}$ les composantes de $b_{j}$ dans la base canonique de $\mathbf{Z}^{g}$. Comme le déterminant de la matrice $\left(b_{1}, \ldots, b_{g}\right)$ est non nul, il existe une permutation $s$ de l'ensemble $\{1, \ldots, g\}$ telle que, pour tout entier $j \in\{1, \ldots, g\}$, on a $b_{s(j), j} \neq 0$. Comme $y$ est réduite au sens de Minkowski, on a $y_{j} \leq$ ${ }^{\mathrm{t}} b_{s(j)} y b_{s(j)}$ et cette dernière quantité vaut $\left\|y^{1 / 2} b_{s(j)}\right\|^{2}$. La première inégalité du lemme 4.21 s'ensuit. Quant à la seconde, observons qu'il revient au même de minorer la plus petite valeur propre de la matrice $M=d^{-1} y d^{-1}$ où $d$ est la matrice diagonale $\operatorname{diag}\left(\sqrt{y_{1}}, \ldots, \sqrt{y_{g}}\right)$. La matrice $M$ appartient à $\mathrm{S}_{g}^{+}$(en particulier ses valeurs propres sont des nombres réels strictement positifs) et ses coefficients sont $m_{i, j}:=y_{i, j} / \sqrt{y_{i} y_{j}}$ (calcul direct). Comme $y$ est réduite au sens de Minkowski, les coefficients de $M$ sont en valeur absolue majorés par $1 / 2$ sauf sur la diagonale où ils valent 1 . Le théorème de Gershgorin entraîne que les valeurs propres de $M$ sont majorées par $\max _{i}\left\{\sum_{j}\left|m_{i, j}\right|\right\} \leq(g+1) / 2$. La plus petite valeur propre de $M$ est donc minorée par $\left(\frac{2}{g+1}\right)^{g-1} \operatorname{det}(M)$ et l'assertion (b) s'ensuit en notant que $\operatorname{det}(M)=\operatorname{det}(y) /\left(y_{1} \cdots y_{g}\right) \geq \mathfrak{c}_{g}^{-g}$.

\section{Remarques 4.22 .}

- La constante $c_{9}$ peut être remplacée par $c_{10}=g\left(\frac{g+2}{4}\right)^{g-1} \mathfrak{c}_{g}^{g}$, qui est meilleure pour les grandes valeurs de $g$. Pour voir cela, reprenons le début de l'argument de la preuve du point (b) ci-dessus. Munissons $\mathrm{M}_{g}(\mathbf{R})$ de la norme de Schur $\|X\|_{2}=\sqrt{\operatorname{tr}\left({ }^{\mathrm{t}} X X\right)}$. Au lieu de minorer la plus petite valeur propre de la matrice $M$, on peut observer qu'il suffit d'estimer la norme de $M^{-1}$. Or l'inégalité d'Hadamard permet de majorer chacun des coefficients de la comatrice de $M \operatorname{par}\left(1+\frac{1}{4}(g-2)\right)^{g-1}=\left(\frac{g+2}{4}\right)^{g-1}$ et donc $\left\|M^{-1}\right\|_{2} \leq \frac{g}{\operatorname{det}(M)}\left(\frac{g+2}{4}\right)^{g-1}$. La constante $c_{10}$ s'en déduit.

- En vertu du lemme 4.19, la constante $\mathfrak{c}_{g}^{g}$ est inférieure à $(1+g / 4)^{g}$, qu'il faut comparer avec la constante $\left(\frac{2 g(g-1)}{3}\right)^{g(g-1) / 2}$ donnée par David \& Philippon dans le lemme 6.4 de 24]. Cette amélioration s'explique par l'utilisation du théorème de Minkowski au lieu du théorème d'Hermite (qui en est une version plus faible), employé dans 24].

Revenons à l'espace de Siegel $\mathfrak{S}_{g}$. Le groupe symplectique $\operatorname{Sp}_{2 g}(\mathbf{R})$ agit sur cet espace de la manière suivante :

$$
\forall \sigma=\left(\begin{array}{ll}
a & b \\
c & d
\end{array}\right) \in \operatorname{Sp}_{2 g}(\mathbf{R}), \forall \tau \in \mathfrak{S}_{g}, \sigma \cdot \tau=(a \tau+b)(c \tau+d)^{-1} .
$$

Sous cette action (transitive et holomorphe), l'orbite d'un élément de $\mathfrak{S}_{g}$ contient une matrice particulière, appelée matrice réduite au sens de Siegel. Une telle matrice est par définition un élément $\tau=x+i y=\left(x_{l, j}+i y_{l, j}\right) \in \mathfrak{S}_{g}$ vérifiant les conditions suivantes :

(1) Pour tous $l, j \in\{1, \ldots, g\}$, on a $\left|x_{l, j}\right| \leq 1 / 2$.

(2) Pour tout $\sigma \in \operatorname{Sp}_{2 g}(\mathbf{Z})$, on a $\operatorname{det}(\operatorname{Im}(\sigma \cdot \tau)) \leq \operatorname{det}(\operatorname{Im}(\tau))$. 
(3) La partie imaginaire $y=\operatorname{Im}(\tau)$ est réduite au sens de Minkowski.

Lorsque $g=1$, les nombres complexes $\tau$ réduits au sens de Siegel sont les éléments du domaine fondamental $\{z \in \mathbf{H} ;|\operatorname{Re}(z)| \leq 1 / 2$ et $1 \leq|z|\}$ pour l'action de $\mathrm{SL}_{2}(\mathbf{Z})=\mathrm{Sp}_{2}(\mathbf{Z})$ sur le demi-plan de Poincaré $\mathbf{H}$.

L'intérêt de ces matrices pour démontrer la proposition 4.17 réside dans le fait que si $A$ est une variété abélienne complexe et principalement polarisée, il existe une matrice $\tau$ réduite au sens de Siegel telle que $A(\mathbf{C}) \simeq \mathbf{C}^{g} /\left(\mathbf{Z}^{g}+\tau \mathbf{Z}^{g}\right)$, la polarisation étant alors donnée par la partie imaginaire de $\tau$ :

$$
\left(\xi, \xi^{\prime}\right) \in \mathbf{C}^{g} \times \mathbf{C}^{g} \mapsto{ }^{\mathrm{t}} \bar{\xi}(\operatorname{Im}(\tau))^{-1} \xi^{\prime} .
$$

Cette description concrète de $A(\mathbf{C})$ est le point de départ de la preuve de la proposition 4.17. Auparavant, notons que si $y$ est la partie imaginaire d'une matrice réduite au sens de Siegel, alors le premier coefficient $y_{1,1}$ de $y$ est plus grand que $\sqrt{3} / 2$. C'est un résultat classique (voir lemme 15 , p. 195, de [39]) qui s'obtient à partir de la deuxième condition dans la définition de matrice réduite au sens de Siegel, à l'aide de la matrice

$$
\sigma=\left(\begin{array}{ccc}
0 & 1 & \mathbf{0} \\
-1 & 0 & \\
\mathbf{0} & \mathrm{I}_{2 g-2}
\end{array}\right) \in \mathrm{Sp}_{2 g}(\mathbf{Z}) .
$$

Ajoutons encore à cette remarque une variante d'un résultat de Graftieaux, très proche d'un «lemme matriciel » au sens ci-dessus.

Lemme 4.23. Soit $A$ une variété abélienne principalement polarisée sur $k$. Étant donné un plongement complexe $\sigma: k \hookrightarrow \mathbf{C}$, soit $\tau_{\sigma}$ une matrice réduite au sens de Siegel telle que $A_{\sigma}(\mathbf{C}) \simeq$ $\mathbf{C}^{g} /\left(\mathbf{Z}^{g}+\tau_{\sigma} \mathbf{Z}^{g}\right)$. Alors on a

$$
\frac{1}{[k: \mathbf{Q}]} \sum_{\sigma: k \hookrightarrow \mathbf{C}} \operatorname{tr} \operatorname{Im} \tau_{\sigma} \leq \frac{32 c_{9}}{\pi}\left(h_{F}(A)+7 g^{3}\right)
$$

où $\operatorname{tr} \operatorname{Im} \tau_{\sigma}$ désigne la trace de la partie imaginaire de $\tau_{\sigma}$.

Démonstration. La preuve de ce lemme s'obtient en recopiant mot à mot celle du lemme 2.12 de [33] sauf en ce qui concerne la constante $c(g)$ définie p. 101 de cet article (égalité (14)) qu'il faut remplacer par $c_{9}^{-1}$ (définie dans le lemme 4.21 ci-dessus). L'avant-dernière inégalité de cette preuve* conduit alors à la majoration

$$
\frac{1}{[k: \mathbf{Q}]} \sum_{\sigma: k \hookrightarrow \mathbf{C}} \operatorname{tr} \operatorname{Im} \tau_{\sigma} \leq \frac{32 c_{9}}{\pi}\left(h_{F}(A)+\frac{5}{2} g \log c_{9}+6 g \log 2\right) .
$$

La borne $\mathfrak{c}_{g} \leq 1+g / 4$ entraîne $c_{9} \leq(1+g / 2)^{2 g-1}$, et le lemme 4.23 découle de ces estimations.

Nous sommes maintenant en mesure d'établir la proposition 4.17 .

Démonstration de la proposition 4.17. Fixons $\sigma: k \hookrightarrow \mathbf{C}$ et une matrice $\tau_{\sigma}=x+i y$ réduite au sens de Siegel telle que $A_{\sigma}(\mathbf{C}) \simeq \mathbf{C}^{g} /\left(\mathbf{Z}^{g}+\tau_{\sigma} \mathbf{Z}^{g}\right)$. Notons $\|\cdot\|_{\sigma}$ la norme hermitienne sur $\mathbf{C}^{g}$ induite par la polarisation $\left(\xi, \xi^{\prime}\right) \mapsto^{\mathrm{t}} \bar{\xi}\left(\operatorname{Im} \tau_{\sigma}\right)^{-1} \xi^{\prime}$. L'application $\Phi$ entre $\mathbf{C}^{g}$ muni de ce produit hermitien et l'espace euclidien usuel $\mathbf{R}^{2 g}$ définie par $\Phi\left(a+\tau_{\sigma} b\right)=\left(y^{-1 / 2}(a+x b), y^{1 / 2} b\right)$ est un isomorphisme isométrique qui permet d'identifier $\mathbf{Z}^{g}+\tau_{\sigma} \mathbf{Z}^{g}$ à un réseau de $\mathbf{R}^{2 g}$, de covolume égal à 1 car

$$
\operatorname{det}\left(\begin{array}{cc}
y^{-1 / 2} & \star \\
\mathbf{0} & y^{1 / 2}
\end{array}\right)=1 \text {. }
$$

Considérons alors une famille libre $\left\{\lambda_{1}, \ldots, \lambda_{2 g}\right\}$ de $\mathbf{Z}^{g}+\tau_{\sigma} \mathbf{Z}^{g}$ donnée par le lemme 4.19. Soit $x \in A(k)$ et définissons

Par translation, on a

$$
\rho_{\sigma}:=\min \left\{\|\lambda\|_{\sigma} ; \lambda \in\left(\mathbf{Z}^{g}+\tau_{\sigma} \mathbf{Z}^{g}\right) \backslash\{0\}\right\} .
$$

$$
\begin{aligned}
r_{\sigma}(x) & \leq \frac{1}{2}\left(\left\|\lambda_{1}\right\|_{\sigma}+\cdots+\left\|\lambda_{2 g}\right\|_{\sigma}\right) \\
& \leq g \max _{1 \leq i \leq 2 g}\left\{\left\|\lambda_{i}\right\|_{\sigma}\right\} \leq g c_{2 g}^{g} \rho_{\sigma}^{-2 g+1},
\end{aligned}
$$

*Mentionnons ici une erreur d'écriture dans le texte original [33] : le terme $-5 / 2 \log c(g)$ doit être remplacé par $-(5 / 2) g \log c(g)$. 
d'où

$$
\frac{1}{[k: \mathbf{Q}]} \sum_{\sigma: k \hookrightarrow \mathbf{C}} \log ^{+} r_{\sigma}(x) \leq \log \left(g c_{2 g}^{g}\right)+\frac{(g-1 / 2)}{[k: \mathbf{Q}]} \sum_{\sigma: k \hookrightarrow \mathbf{C}} \log ^{+} \rho_{\sigma}^{-2} .
$$

Soit $\lambda=a+\tau_{\sigma} b$, non nul, avec $a, b \in \mathbf{Z}^{g}$. Par définition, on a

$$
\begin{aligned}
\|\lambda\|_{\sigma}^{2} & =\left\|a+\operatorname{Re}\left(\tau_{\sigma}\right) b\right\|_{\sigma}^{2}+\left\|\operatorname{Im}\left(\tau_{\sigma}\right) b\right\|_{\sigma}^{2} \\
& =\left\|a+\operatorname{Re}\left(\tau_{\sigma}\right) b\right\|_{\sigma}^{2}+{ }^{t} b \operatorname{Im}\left(\tau_{\sigma}\right) b .
\end{aligned}
$$

- Si $b$ est non nul alors on a $\|\lambda\|_{\sigma}^{2} \geq{ }^{\mathrm{t}} b \operatorname{Im}\left(\tau_{\sigma}\right) b \geq \frac{\sqrt{3}}{2}$.

- Si $b$ est nul alors on a $\|\lambda\|_{\sigma}^{2}={ }^{\mathrm{t}} a\left(\operatorname{Im} \tau_{\sigma}\right)^{-1} a \geq \tau_{\max }^{-1}$ où $\tau_{\max }$ est la plus grande valeur propre de $\operatorname{Im} \tau_{\sigma}$.

Compte tenu de ces deux cas, nous avons

$$
\rho_{\sigma}^{2} \geq \min \left\{\frac{\sqrt{3}}{2}, \frac{1}{\operatorname{tr} \operatorname{Im} \tau_{\sigma}}\right\}
$$

et, comme $\operatorname{tr} \operatorname{Im} \tau_{\sigma} \geq \frac{\sqrt{3}}{2}$, nous obtenons

$$
\max \left\{1, \rho_{\sigma}^{-2}\right\} \leq\left(\frac{2}{\sqrt{3}}\right)^{2} \operatorname{tr} \operatorname{Im} \tau_{\sigma} .
$$

L'inégalité (44), la concavité du logarithme et le lemme 4.23 entraînent la majoration

$$
\begin{aligned}
& \frac{1}{[k: \mathbf{Q}]} \sum_{\sigma: k \hookrightarrow \mathbf{C}} \log ^{+} r_{\sigma}(x) \\
& \quad \leq \log \left(g c_{2 g}^{g}\right)+(g-1 / 2)\left(2 \log \frac{2}{\sqrt{3}}+\log \frac{32 c_{9}}{\pi}+\log \left(h_{F}(A)+7 g^{3}\right)\right) \\
& \quad \leq g \log ^{+}\left\{h_{F}(A)\right\}+c_{11}
\end{aligned}
$$

avec

$$
\begin{gathered}
c_{11}:=\log (g)+(g-1 / 2) \log \frac{128}{3 \pi}+(g-1 / 2)(g-1) \log \frac{g+1}{2} \\
+2 g^{2} \log (1+g / 2)+(g-1 / 2) \log \left(1+7 g^{3}\right) .
\end{gathered}
$$

La proposition 4.17 s'ensuit en observant à la suite d'un calcul élémentaire que $c_{11} \leq 5 g^{2} \log (g+$ 1).

Remarque 4.24. À travers la majoration (43) de la démonstration, nous avons utilisé le théorème de Minkowski sous la forme simplifiée

$$
\left\|\lambda_{1}\right\|_{\sigma}^{2 g-1}\left\|\lambda_{2 g}\right\|_{\sigma} \leq \mathfrak{c}_{2 g}^{g} .
$$

Cela nous a permis d'estimer la norme de $\lambda_{2 g}$ en fonction de celle de $\lambda_{1}$, qui, dans le cas présent, était contrôlée par le théorème de Graftieaux. Comme me l'a fait remarqué le rapporteur, il aurait été plus astucieux de tenir compte du caractère autodual du réseau $\mathbf{Z}^{g}+\tau_{\sigma} \mathbf{Z}^{g}$ relativement à la polarisation principale donnée par $L_{\sigma}$. On peut alors remplacer (45) par une inégalité de transférence qui relie le premier minimum d'un réseau au dernier du réseau dual. Par exemple, le travail [3] de Banaszczyk donne alors l'estimation

$$
\left\|\lambda_{1}\right\|_{\sigma}\left\|\lambda_{2 g}\right\|_{\sigma} \leq 2 g
$$

qui permettrait, in fine, de diviser au moins par $g$ le majorant de l'inégalité (42) de la proposition 4.17. Ce raffinement n'a aucun conséquence significative sur la constante $c_{5}$ du théorème 3.1 (voir la deuxième ligne de l'inégalité (112), p. 57; le nombre $20 g^{3}(g+t)$ serait divisé par $g$ et il resterait négligeable devant les autres termes). 


\section{DÉmonstration DU THÉORÈme 3.1}

5.1. Description de la preuve. La démonstration du théorème 3.1 comporte plusieurs étapes et elle s'avère globalement assez longue. Notre objectif ici est d'effectuer un survol général de la preuve en expliquant la démarche et en en dégageant les principales caractéristiques dont, en particulier, le procédé de réduction d'Hirata-Kohno.

La preuve du théorème 3.1 s'articule autour de l'inégalité de pentes du lemme 4.12 (voir les notations p. 19):

$$
\widehat{\mu}(\overline{\mathcal{E}}) \leq \sum_{i=1}^{N} \frac{\operatorname{dim}\left(E_{i} / E_{i+1}\right)}{\operatorname{dim} E}\left(\widehat{\mu}_{\max }\left(\overline{\mathcal{G}}_{i}\right)+h\left(\varphi_{i}\right)\right)
$$

Nous allons voir dans un instant comment choisir les fibrés vectoriels hermitiens $\overline{\mathcal{E}},\left(\overline{\mathcal{G}}_{i}\right)_{i}$ sur $\operatorname{Spec} \mathcal{O}_{K}$ et les applications $K$-linéaires $\left(\varphi_{i}\right)_{i}$ (où $K$ est une certaine extension finie de $k$ ). Toutefois, pour éclaircir la discussion, il est important de mentionner dès à présent que la distance $\mathrm{d}(u, W)$ à laquelle nous nous intéressons n'apparaîtra que dans les majorations « fines » des normes $\left\|\varphi_{i}\right\|_{\sigma}$, où $\sigma$ est une place quelconque de $K$ au-dessus de $\sigma_{0}$. De la sorte, pourvu que l'on puisse justifier l'injectivité de $\varphi$, l'inégalité de pentes (46) fournira une minoration de cette distance. Il en ressort aussi que la «qualité » de la mesure dépend en grande partie de l'évaluation des quantités $\widehat{\mu}_{\max }\left(\overline{\mathcal{G}}_{i}\right)$ et $\left\|\varphi_{i}\right\|_{v}\left(v \nmid \sigma_{0}\right)$. Une estimation trop brutale de l'un de ces termes peut compromettre certains aspects théoriques importants de la mesure du théorème 3.1, comme, par exemple, la linéarité en $\log b$.

Les meilleures mesures connues actuellement dans le cadre abélien 23, 28, ont été obtenues au moyen de la méthode de Baker, de la réduction d'Hirata-Kohno et du procédé de changement de variables de Chudnovsky. Ces techniques de l'approximation diophantienne guident le choix des objets $\overline{\mathcal{E}},\left(\overline{\mathcal{G}}_{i}\right)_{i},\left(\varphi_{i}\right)_{i}$, comme nous allons l'esquisser maintenant.

Pour ne pas alourdir les notations, nous considérons ici seulement le cas d'une variété abélienne (i.e. $n=1$ ). Soit $S_{0}<S, T, D_{0}, D_{1}$ des entiers naturels qui jouent le rôle de paramètres à choisir « convenablement ». Pour des raisons pratiques, ces choix sont donnés au début de la preuve ( $(5.3)$ et ils permettent de réaliser de manière optimale (aux constantes près) les différentes conditions qui interviennent au cours de la preuve. Nous introduisons la compactification $X=X_{0} \times A$ du groupe algébrique $G$ et le fibré en droites $M=\mathcal{O}_{X_{0}}\left(D_{0}\right) \otimes L^{\otimes 3 D_{1}}$ sur $X$. Notons $E:=\mathrm{H}^{0}(X, M)$. À partir d'un modèle de Moret-Bailly de $\left(A, L,(m p)_{0 \leq m \leq(g+t) S}\right)$, nous formons des modèles entiers et hermitiens de ces données, à savoir une variété arithmétique $\mathcal{X}$ (quasi-projective) sur $\operatorname{Spec} \mathcal{O}_{K}$, un fibré en droites hermitien $\overline{\mathcal{M}}$ sur $\mathcal{X}$ puis le fibré vectoriel hermitien $\overline{\mathcal{E}}=\overline{\mathrm{H}^{0}(\mathcal{X}, \mathcal{M})} \operatorname{sur} \operatorname{Spec} \mathcal{O}_{K}$, de fibre générique $E \otimes_{k} K$. Le morphisme $\varphi$ est obtenu par restriction de $\mathcal{M}_{K}$ le long d'un sousschéma $\mathbf{T}$ de $\mathcal{X}_{K}$ de dimension nulle, constitué de voisinages infinitésimaux autour des multiples $m p, 0 \leq m \leq(g+t) S$, du point $p$, dans les directions de $W$ jusqu'à l'ordre $2(g+t) T$ si $m \leq S_{0}$ et $(g+t) T$ si $S_{0}<m \leq(g+t) S$. Le schéma $\mathbf{T}$ est en quelque sorte une collection de « points épaissis ». Ces choix résulte de la méthode de Baker dont nous rappelons brièvement le principe général. On fixe une direction privilégiée de $W \otimes_{\sigma_{0}} \mathbf{C}$, en l'occurrence ici un vecteur $w \in W \otimes_{\sigma_{0}} \mathbf{C}$ tel que $\mathrm{d}(u, W)=\|u-w\|_{\sigma_{0}}$. On se restreint alors à la droite complexe engendrée par $w$ pour n'avoir à considérer que des fonctions entières d'une seule variable complexe. Étant donné une telle fonction $f$ qui s'annule à l'ordre $2(g+t) T$ en un nombre fini de points complexes (pour nous ce seront les $m u, 0 \leq m \leq S_{0}$ ), un lemme d'interpolation (comme le lemme 5.14, p. 49) permet d'en déduire une majoration des dérivées de $f$ à un ordre deux fois plus faible, $(g+t) T$, mais en d'autres points de $\mathbf{C}$ (ici, $m u, S_{0}<m \leq(g+t) S$ ). Cette observation de Baker, qui a donné le jour à la méthode du même nom, justifie le choix de $\mathbf{T}$ et aussi celui de la filtration qui intervient dans la méthode des pentes. Cette dernière peut se décrire de la manière suivante. Étant donné $s \in E \otimes_{\sigma_{0}} \mathbf{C}$ et un entier $m \in\{0, \ldots,(g+t) S\}$, nous annulons un à un les jets de $s$ le long de $W$ au point $m p$ jusqu'à l'ordre $2(g+t) T$ ou $(g+t) T$ selon que $m \leq S_{0}$ ou non. Puis, une fois le dernier jet au point $m p$ annulé, nous recommençons la même procédure à partir du point suivant $(m+1) p$. L'ordre d'annulation est lexicographique sur l'ensemble des couples $(m, \ell)$ correspondant au point $m p$ et à l'ordre d'annulation $\ell$ le long de $W$. De cette manière, le morphisme $\varphi_{i}$ associe à une section $s$ de $\mathcal{M}_{K}$, dont les jets d'ordre $\ell^{\prime}$ au point $m^{\prime} p$ sont nuls pour $\left(m^{\prime}, \ell^{\prime}\right) \preccurlyeq(m, \ell)$ (où $\preccurlyeq$ désigne l'ordre lexicographique sur $\left.\mathbf{N}^{2}\right)$, le jet d'ordre $\ell$ le long de $W$ au point $m p(\ell$ et $m$ sont 
des entiers naturels attachés à $i$ ). Ainsi définie, l'application $\varphi_{i}$ est «intrinsèque ». Quant au fibré hermitien $\overline{\mathcal{G}}_{i}$, sa fibre générique s'identifie à $S^{\ell}\left(W^{\vee}\right) \otimes(m p)^{*} \mathcal{M}_{K}$, espace d'accueil naturel du jet d'ordre $\ell$ le long de $W$ au point $m p$ d'une section de $\mathcal{M}_{K}$.

Après avoir construit ces objets, la difficulté consiste à évaluer précisément chacune des quantités $\widehat{\mu}_{\max }\left(\overline{\mathcal{G}}_{i}\right),\left\|\varphi_{i}\right\|_{v}(v$ place de $K)$ et $\widehat{\mu}(\overline{\mathcal{E}})$ et à s'assurer que l'application $\varphi$ est injective. Ce schéma de preuve est, somme toute, relativement classique dans un contexte «inégalités de pentes ». Hormis peut-être le choix de $\mathbf{T}$ et de la filtration «à la Baker », il est largement inspiré des travaux de Bost [10, 12] et Graftieaux [33, 34]. Toutefois, si la méthode des pentes telle que nous venons de l'expliquer peut permettre d'obtenir des estimations explicites de formes linéaires de logarithmes, il est indispensable également de présenter le problème sous le «bon » angle afin que la mesure obtenue soit satisfaisante. C'est ce que nous allons expliquer maintenant en évoquant la réduction d'Hirata-Kohno. Auparavant, oublions momentanément le groupe affine $G_{0}$ et le sousespace $W$ introduits au $\S 3.1$. Lorsque l'on s'intéresse à des formes linéaires de logarithmes abéliens, la situation naturelle au départ comprend la donnée d'un logarithme $u$ d'un point $p \in A(\overline{\mathbf{Q}})$ et d'un sous-espace $W_{0}$ de l'espace tangent $t_{A}$. La question qui se pose est de minorer la distance de $u$ à $W_{0} \otimes_{\sigma_{0}}$ C. Il serait donc naturel de considérer les sections globales du fibré en droites $L^{\otimes 3 D_{1}}$ et leurs jets le long de $W_{0}$ à différents ordres en les multiples du point $p$ comme ci-dessus. Or il s'avère que si nous procédons de cette manière, il est impossible d'avoir une minoration de $\log \mathrm{d}(u, W)$ linéaire en $\log b$ (avec le groupe standard $\mathbf{H}=\{0\}$ ). Il y a au moins deux raisons à cela. La pente maximale du fibré vectoriel hermitien $\overline{\mathcal{G}}_{i}$ (dont, ici, la fibre générique serait $S^{\ell}\left(W_{0}^{\vee}\right) \otimes(m p)^{*} L^{\otimes 3 D_{1}}$ ) serait fonction de $T \log b$ (d'après les estimation (32) et (33), p. 18) et les estimations ultramétriques des normes des $\varphi_{i}$ feraient intervenir $T$ !. Elles apporteraient alors une contribution* en $T \log T$. De la sorte, on ne pourrait avoir mieux que

$$
\log \mathrm{d}(u, W) \geq-T(\log b+\log T) .
$$

Par ailleurs, des contraintes liées à la condition de Siegel $T^{\operatorname{dim} W_{0}} S \simeq D_{1}^{g}$, qui assure l'injectivité de $\varphi$, entraînent une dépendance polynomiale en $\log b$ du paramètre $T$, et, a fortiori, de $T(\log b+\log T)$. Par conséquent, le problème formulé à l'état brut débouche sur une mesure qui ne convient pas (ou plus) vis-à-vis du paramètre $\log b$. Mentionnons néanmoins son caractère historique puisqu'il s'agit de la voie empruntée par Philippon \& Waldschmidt dans leur article fondateur [57]. Il faut donc transformer le problème initial pour améliorer la dépendance en $\log b$ et c'est l'objet de la réduction d'Hirata-Kohno. Géométriquement, cette idée consiste à adjoindre à la variété abélienne le groupe affine $G_{0}=\mathbf{V}\left(\left(t_{A} / W_{0}\right)^{\vee}\right)$ pour former le groupe algébrique $G=G_{0} \times A$, puis de considérer le sous-espace vectoriel «transversal $W \subseteq t_{G}=t_{G_{0}} \oplus t_{A}$ obtenu à partir du graphe de la projection $t_{A} \rightarrow t_{A} / W_{0}$. Le premier avantage de cette transformation des données $\left(A, W_{0}\right)$ en $(G, W)$ est la possibilité d'avoir un paramètre supplémentaire $D_{0}$ attaché au schéma en groupes $G_{0}$, qui apporte plus de souplesse pour réaliser les différentes contraintes qui surviennent au cours de la preuve. Mais, assurément, le plus remarquable est qu'il devient possible de faire porter le poids des dérivations le long de $W$ sur le groupe affine $G_{0}$. En effet, la transformation effectuée opère à la fois sur la pente maximale de $\overline{\mathcal{G}}_{i}$ qui admet désormais un majorant indépendant de $\log b$ (voir la proposition 5.8, p. 39) et sur la pente d'Arakelov de $\overline{\mathcal{E}}$ qui, elle, décroît d'un facteur $-D_{0} \log b$ (à une constante près, voir la proposition 5.5, p. 377. De cette manière, la quantité $T \log b$ a été remplacée par $D_{0} \log b$. Si l'on s'arrête à cette réduction, il reste encore le terme en $T \log T$ qui conduit à une mesure qui n'est pas linéaire en $\log b$ mais qui comporte une puissance supplémentaire de $\log \log b$, comme dans les résultats présentés dans [35, 36]. Ce terme $T \log T$ provient d'une estimation assez grossière des normes ultramétriques des jets de section qui entrent en jeu au cours de la preuve, puisque, pour rendre « entier » un tel jet d'ordre $\ell$, l'on multipliait par $\ell$ ! David \& Hirata-Kohno ont redécouvert récemment dans leur article 23] comment une méthode basée sur un changement de variables, due à Chudnovsky [17], permet d'affiner ces estimations $p$-adiques en remplaçant ainsi $T \log T$ par $T \log D_{0}$. La mise en œuvre de ce procédé s'appuie sur les techniques décrites dans [28] et rappelées dans la démonstration de la proposition 5.10 du $\S 5.8$. Elle va de pair avec la réduction d'Hirata-Kohno.

Pour clore ce paragraphe, évoquons brièvement les différentes étapes de la démonstration du théorème 3.1. Une première réduction permet de se ramener à une variété abélienne principalement

\footnotetext{
${ }^{*}$ Mentionnons que cette difficulté technique est aussi au centre du théorème 3.4 de 12 (voir le début du $\S 4$ de cet article) et des résultats de Graftieaux 33, 34.
} 
polarisée et de disposer de la sorte du lemme 4.16 et de la proposition 4.17 sous leurs formes entièrement explicites. Puis, au $\S 5.3$, nous présentons les paramètres $S_{0}, S, T, D_{0}, D_{1}, \ldots$ utilisés au cours de la preuve et nous énonçons une généralisation du théorème 3.1 (il s'agit du théorème 5.2) qui fera l'objet de la démonstration proprement dite. Le $\S 5.4$ contient un résultat technique équivalent à l'injectivité du morphisme $\varphi$ qui sera défini un peu plus tard, au $\S 5.6$. Les objets $\overline{\mathcal{E}},\left(\overline{\mathcal{G}}_{i}\right)_{i},\left(\varphi_{i}\right)_{i}$ sont introduits aux $\S \S 5.5$ et 5.6, accompagnés des propriétés indispensables requises dans la suite. Le $\S 5.7$ est une reformulation de la condition de Siegel évoquée ci-dessus. Il vise à établir que pour un certain entier $i_{0}$ « assez » grand (mais bien défini), la dimension du sousespace $E_{i_{0}}$ de $E$ est encore du même ordre que celle de $E$. Cela interviendra dans l'analyse ultime du résultat fourni par l'inégalité de pentes (46) au $\S$ 5.11. Les estimations des normes de morphismes $\varphi_{i}$ aux différentes places font l'objet des $\S \S 5.8$ à 5.10. Enfin, en conclusion ( $\S 5.11$ ), nous regroupons tous les résultats intermédiaires obtenues dans l'inégalité de pentes (46). Grâce à un raisonnement par l'absurde, nous en déduisons le théorème 5.2 énoncé au $\S 5.3$ et nous expliquons comment le théorème 3.1 en découle. Nous concluons avec la démonstration des résultats énoncés dans l'introduction.

5.2. Remarque et réduction préliminaires. Au $\S 3.1$, nous avons introduit un corps de nombres $k$ sur lequel tous les objets algébriques $A, p, W$ etc. qui interviennent dans l'énoncé du théorème 3.1 sont définis. Toutefois, pour effectuer la démonstration, il est nécessaire de se placer sur une extension finie $K \mid k$ pour laquelle nous disposons d'un modèle de Moret-Bailly de $\left(A, L,(m p)_{0 \leq m \leq(g+t) S}\right.$ ) (voir le début du $\S$ 5.5). Il est important de noter que cela ne constitue en rien une difficulté technique car le degré relatif $[K: k]$ disparaîtra à la fin de la preuve lorsque nous appliquerons l'inégalité de pentes (35). Le corps $K$ peut donc être choisi arbitrairement grand. La raison en est que les estimations locales des normes des morphismes $\varphi_{i}$ sont identiques en toutes les places $v$ de $K$ au-dessus d'une même place de $k$.

Par ailleurs, nous supposerons jusqu'à la fin de la démonstration que la variété abélienne $(A, L)$ est principalement polarisée, c'est-à-dire que la dimension de l'espace des sections $\mathrm{H}^{0}(A, L)$ vaut 1. Cette hypothèse ne restreint pas la portée du théorème 3.1. En effet, après une éventuelle extension finie du corps $k$ (qui est sans conséquence comme nous venons de le voir), il est possible de se ramener à ce cas en considérant, pour tout entier $i \in\{1, \ldots, n\}$, une isogénie $\varsigma_{i}:\left(A_{i}, L_{i}\right) \rightarrow\left(A_{i}^{\prime}, L_{i}^{\prime}\right)$, de degré $\mathrm{h}^{0}\left(A_{i}, L_{i}\right)$, entre la variété polarisée $\left(A_{i}, L_{i}\right)$ et une variété abélienne principalement polarisée $\left(A_{i}^{\prime}, L_{i}^{\prime}\right)$. Une telle isogénie peut être construite en quotientant par un sous-groupe lagrangien du groupe de Mumford de $\left(A_{i}, L_{i}\right)$ (voir [53], p. 234). Le fibré en droites $L_{i}^{\prime}$ conserve ainsi les propriétés d'amplitude et de symétrie de $L_{i}$. La variété abélienne $A^{\prime}:=A_{1}^{\prime} \times \cdots \times A_{n}^{\prime}$ munie du fibré en droites $L^{\prime}:=L_{1}^{\prime} \otimes \cdots \otimes L_{n}^{\prime}$ est principalement polarisée. Notons $\varsigma: A \rightarrow A^{\prime}$ l'isogénie induite par les $\varsigma_{i}$ et $\mathrm{d} \varsigma: t_{A} \rightarrow t_{A^{\prime}}$ sa différentielle à l'origine. Les données initiales doivent être modifiées et remplacées par le système «image » par $\varsigma$ :

$$
\left\{A_{i}^{\prime}, L_{i}^{\prime}, \varsigma_{i}\left(p_{i}\right), \varsigma(\mathrm{H}), \mathrm{d} \varsigma_{i} \cdot\left(u_{i}\right), \mathrm{d} \varsigma .\left(W_{0}\right), \mathrm{d} \varsigma \cdot\left(u_{0}\right)\right\} .
$$

Les invariants liés à ces nouvelles données sont contrôlés de la manière suivante. En vertu de l'estimation de Raynaud (22), on a

$$
\forall i \in\{1, \ldots, n\}, \quad h_{F}\left(A_{i}^{\prime}\right) \leq h_{F}\left(A_{i}\right)+\frac{1}{2} \log \mathrm{h}^{0}\left(A_{i}, L_{i}\right)
$$

et, par définition, on a aussi $\operatorname{deg}_{L^{\prime}} \varsigma(\mathrm{H})=\operatorname{deg}_{L} \mathrm{H}, \widehat{h}_{L_{i}^{\prime}}\left(\varsigma_{i}\left(p_{i}\right)\right)=\widehat{h}_{L_{i}}\left(p_{i}\right)(\forall i)$. Le choix des métriques liées à $L\left(\right.$ resp. $\left.L^{\prime}\right)$ sur l'espace tangent $t_{A}\left(\right.$ resp. $\left.t_{A^{\prime}}\right)$ assure leurs compatibilités et la propriété d'isométrie de d $\varsigma$ aux places infinies du corps de nombres ambiant. Il suffit donc d'établir le théorème 3.1 avec les données (47) ou, ce qui revient au même, en supposant que $(A, L)$ est principalement polarisée.

5.3. Choix des paramètres et généralisation de l'énoncé 3.1. Revenons aux notations du $\S 3.1$, avec l'hypothèse de principalité de $(A, L)$. Dans ce paragraphe, nous allons donner de manière explicite les paramètres qui conduisent au théorème 3.1. Ces choix ne sont pas absolument essentiels pour comprendre les principaux arguments utilisés dans la suite mais ils vont permettre de formuler et de prouver un énoncé un peu plus général que le théorème 3.1.

En préambule, rappelons qu'à une sous-variété $V$ de $\mathbf{P}_{\bar{k}}$ lui est associé un polynôme dit de Hilbert-Samuel, noté $H_{V}$. Soit $\mathscr{H}\left(V ; X_{0}, \ldots, X_{n}\right)$ la partie homogène de plus haut degré $(=\operatorname{dim} V)$ 
de $H_{V}$ multipliée par ( $\left.\operatorname{dim} V\right)$ !. Ce dernier polynôme possède quelques propriétés qui interviendront de manière régulière dans la suite :

(1) Tous les coefficients de $\mathscr{H}\left(V ; X_{0}, \ldots, X_{n}\right)$ sont des entiers positifs et leur somme vaut $\operatorname{deg} V$.

(2) Si $V$ est un produit $V_{0} \times \cdots \times V_{n}$ où $V_{i}$ est une sous-variété de la $i^{\text {ème }}$ composante de $\mathbf{P}_{\bar{k}}$, alors

$$
\mathscr{H}\left(V ; X_{0}, \ldots, X_{n}\right)=(\operatorname{dim} V) ! \prod_{i=0}^{n} \frac{\mathscr{H}\left(V_{i} ; X_{i}\right)}{\left(\operatorname{dim} V_{i}\right) !} .
$$

(3) Si $\kappa \in\{0, \ldots, n\}$ et si $\pi_{\kappa}$ désigne la projection canonique de $\mathbf{P}_{\bar{k}}$ sur ses $n-\kappa+1$ derniers facteurs, alors, pour tout $V \subseteq \mathbf{P}_{\bar{k}}$ et tout $\left(x_{0}, \ldots, x_{n}\right) \in\left[1,+\infty\left[{ }^{n+1}\right.\right.$, on a

$$
\left(\begin{array}{c}
\operatorname{dim} V \\
\operatorname{dim} \pi_{\kappa}(V)
\end{array}\right) \mathscr{H}\left(\pi_{\kappa}(V) ; x_{\kappa}, \ldots, x_{n}\right) \leq \mathscr{H}\left(V ; x_{0}, \ldots, x_{n}\right) .
$$

(4) Pour notre groupe algébrique $G$, on a

$$
\mathscr{H}\left(G ; X_{0}, \ldots, X_{n}\right)=3^{g} \frac{(g+t) !}{t !} X_{0}^{t} X_{1}^{g_{1}} \cdots X_{n}^{g_{n}} .
$$

(5) Pour tout sous-groupe algébrique connexe $G^{\prime}$ de $G$ et tout $(n+1)$-uplet $\left(x_{0}, \ldots, x_{n}\right) \in$ ] $0,+\infty\left[{ }^{n+1}\right.$, l'application partielle

$$
x_{i} \longmapsto \frac{\mathscr{H}\left(G^{\prime} ; x_{0}, \ldots, x_{n}\right)}{\mathscr{H}\left(G ; x_{0}, \ldots, x_{n}\right)}
$$

est décroissante.

Nous renvoyons le lecteur au texte de Rémond [60] pour une présentation plus systématique de la fonction $\mathscr{H}$. Après ces rappels, introduisons cinq constantes 《fondamentales 》 还, ..., 且 à partir desquelles d'autres constantes seront définies par la suite. Soit

$$
\begin{aligned}
& \text { 相: }=(10 g)^{4}(g+t) n \text {, } \\
& \text { 吗: }:=2^{3 g+t} 3^{2 g} g !(g+t) !(2(g+t)+1)^{g}(g+t)^{2} \text {, } \\
& \text { 214: }=100 \times 18^{g}\left(\frac{(g+t) !}{t !}\right)^{2} \text {, } \\
& \text { 成: }=100(48 \pi+6)(g+t) n c \text { 有 } \\
& \text { 迫: }=(12)^{g} \frac{(g+t) !}{t !}
\end{aligned}
$$

Les contraintes qui ont motivé ces choix sont évoquées dans l'appendice. Définissons alors les entiers $S_{0}:=c_{12} \mathfrak{a}$ et $S:=c_{13} S_{0}-1$. Soit $U_{0}$ un nombre réel strictement positif (défini un peu après) et considérons les nombres réels $\widetilde{D}_{0}, \ldots, \widetilde{D}_{n}, \widetilde{T}$ donnés par les formules

$$
\widetilde{T}:=\frac{U_{0}}{S_{0} \log \mathfrak{e}}, \quad \widetilde{D}_{0}:=\frac{U_{0}}{c_{14}\left(\log b+S_{0}^{y} \log \mathfrak{e}\right)}
$$

(l'entier $y \in\{0,1\}$ a été introduit au $\S$ 3.2) et

$$
\forall i \in\{1, \ldots, n\}, \quad \widetilde{D}_{i}:=\frac{U_{0}}{c_{15}\left(S_{0} \log \mathfrak{e}\right)\left(1+\frac{D S_{0}}{\log \mathfrak{e}} \log a_{i}\right)} .
$$

La décroissance des $a_{i}$ entraîne $\widetilde{D}_{1} \leq \cdots \leq \widetilde{D}_{n}$. Par ailleurs, un sous-groupe algébrique connexe $G^{\prime}$ de $G_{\bar{k}}$ se décompose en $G_{0}^{\prime} \times A^{\prime}$ où $G_{0}^{\prime}\left(\right.$ resp. $\left.A^{\prime}\right)$ est un sous-groupe algébrique connexe de $G_{0, \bar{k}}$ (resp. de $A_{\bar{k}}$ ). Pour un tel $G^{\prime}$, définissons les entiers

$$
\begin{aligned}
& t^{\prime}:=\operatorname{dim} G_{0}^{\prime}, \quad g^{\prime}:=\operatorname{dim} A^{\prime}, \quad d^{\prime}:=\operatorname{dim} G^{\prime}=t^{\prime}+g^{\prime}, \\
& r^{\prime}:=\operatorname{codim}_{G_{\bar{k}}} G^{\prime}, \quad \lambda^{\prime}:=\operatorname{codim}_{W_{\bar{k}}}\left(W_{\bar{k}} \cap t_{G^{\prime}}\right) .
\end{aligned}
$$

En minorant $\operatorname{dim}\left(t_{G_{0}^{\prime}}+\lambda\left(t_{A^{\prime}}\right)\right)$ par $\max \left\{t^{\prime}, \operatorname{dim} \lambda\left(t_{A^{\prime}}\right)\right\}$, nous obtenons la relation

$$
r^{\prime}-\lambda^{\prime}=t-\operatorname{dim}\left(t_{G_{0}^{\prime}}+\lambda\left(t_{A^{\prime}}\right)\right) \leq \min \left\{t-t^{\prime}, g-g^{\prime}\right\},
$$


qui sera utilisée plusieurs fois dans la suite. En particulier, l'entier $\varrho_{\mathrm{H}}$ défini par (10) (p. 9) est lié à $\lambda_{\mathrm{H}}=\operatorname{codim}_{W_{\bar{k}}}\left(W_{\bar{k}} \cap t_{\mathrm{H}}\right)$ à travers la formule $\lambda_{\mathrm{H}}=\varrho_{\mathrm{H}}+g-\operatorname{dim} \mathrm{B}$. On a également $\varsigma_{\mathrm{H}}=r_{\mathrm{H}}-\lambda_{\mathrm{H}}$. Lorsque $W_{\bar{k}}+t_{G^{\prime}} \neq t_{G_{\bar{k}}}\left(\right.$ i.e. $\left.r^{\prime} \neq \lambda^{\prime}\right)$, posons

$$
x\left(G^{\prime}\right):=\frac{1}{\widetilde{D}_{0}}\left(\frac{\widetilde{T}^{\lambda^{\prime}}(S+1) \mathscr{H}\left(A^{\prime} ; \widetilde{D}_{1}, \ldots, \widetilde{D}_{n}\right)}{c_{16} \mathscr{H}\left(A ; \widetilde{D}_{1}, \ldots, \widetilde{D}_{n}\right)}\right)^{\frac{1}{t-t^{\prime}}} .
$$

Observons alors que l'ensemble $\left\{x\left(G^{\prime}\right) ; x\left(G^{\prime}\right) \leq x(\{0\})\right\}$ est fini. En effet, il n'existe qu'un nombre fini de possibilités pour les entiers $r^{\prime}$ et $\lambda^{\prime}$ et la majoration $x\left(G^{\prime}\right) \leq x(\{0\})$ entraîne une majoration du degré de $A^{\prime}$. Grâce à la propriété (1) de la fonction $\mathscr{H}$, il n'y a donc qu'un nombre fini de possibilités pour $\mathscr{H}\left(A^{\prime} ; \widetilde{D}_{0}, \ldots, \widetilde{D}_{n}\right)$ puis finalement pour $x\left(G^{\prime}\right)$. Nous pouvons alors définir le nombre réel

$$
x:=\min \left\{x\left(G^{\prime}\right)\right\}>0
$$

où $G^{\prime}$ parcourt les sous-groupes algébriques connexes de $G_{\bar{k}}$ tels que $W_{\bar{k}}+t_{G^{\prime}} \neq t_{G_{\bar{k}}}$. Dans la suite, il est commode de fixer un tel sous-groupe $\widetilde{G}=\widetilde{G}_{0} \times \widetilde{A}$ pour lequel $x=x(\widetilde{G})$, auquel on adjoint les entiers $\tilde{t}, \widetilde{g}, \widetilde{d}, \widetilde{\lambda}$ définis par $(48)$. En outre, puisque $\mathscr{H}\left(A^{\prime} ; \widetilde{D}_{0}, \ldots, \widetilde{D}_{n}\right)\left(\operatorname{resp} . \mathscr{H}\left(A ; \widetilde{D}_{0}, \ldots, \widetilde{D}_{n}\right)\right)$ est un polynôme homogène de degré $g^{\prime}$ (resp. $g$ ), le nombre $U_{0}^{\left(r^{\prime}-\lambda^{\prime}\right) /\left(t-t^{\prime}\right)} x\left(G^{\prime}\right)$ ne dépend pas du choix de $U_{0}$. Ainsi, compte tenu de (49) et de l'hypothèse $\varsigma_{\mathrm{H}}>0$, nous pouvons choisir $U_{0}$ de manière à ce que $x(\mathrm{H})$ vaille 1 (voir (98), p. 51, pour une formule explicite). En particulier, on a $x \leq 1$.

Notons $T:=[\widetilde{T}], D_{0}:=\left[x \widetilde{D}_{0}\right], D_{i}:=\left[\widetilde{D}_{i}\right]$ pour $1 \leq i \leq n$ et $D_{i}^{\prime}:=\max \left\{1, D_{i}\right\}$ pour $0 \leq i \leq n$. Précisons que dans la démonstration qui va suivre, les $D_{i}$ seront des degrés (partiels) de polynômes, $T$ sera un «ordre de dérivation », $S$ un «nombre de points » dans le procédé d'extrapolation et $x$ une « variable d'ajustement » pour assurer l'injectivité d'un certain morphisme d'évaluation. Le lemme suivant résume les principales relations que doivent satisfaire ces paramètres entre eux.

Lemme 5.1. Les inégalités suivantes sont vérifiées.

(i) $T \geq 0 \sqrt{15} D_{n}$ et $T \geq 1$.

(ii) $T \geq 0 D_{0} /\left(2 S_{0}^{1-y}\right)$.

(iii) $D_{0} \geq 1$ et $D_{n} \geq 1$.

(iv) $2(g+t) T \log \left(4 D_{0}\right) \leq U_{0} /(50 D)$.

(v) $\forall i \in\{1, \ldots, n\},(g+t)^{2} S^{2} D_{i}\left(\mathfrak{e}\left\|u_{i}\right\|_{\sigma_{0}}\right)^{2} \leq c_{17} U_{0}$ où

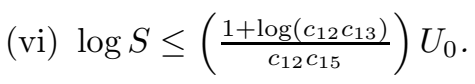

$$
\text { 1] }:=\frac{(g+t)^{2} q_{13}^{2}}{c \sqrt{15}}=\frac{(g+t)}{100(48 \pi+6) n} \text {. }
$$

Démonstration. La première inégalité est une conséquence immédiate des choix des paramètres $T$ et $D_{n}$ puisque $\widetilde{T} \geq \sqrt{15} \widetilde{D}_{n}$. Pour la suivante, $T \geq 1$, la propriété (5) de $\mathscr{H}$ implique

$$
\frac{\mathscr{H}\left(\mathrm{B} ; \widetilde{D}_{1}, \ldots, \widetilde{D}_{n}\right)}{\mathscr{H}\left(A ; \widetilde{D}_{1}, \ldots, \widetilde{D}_{n}\right)} \geq \frac{\mathscr{H}\left(\mathrm{B} ; \widetilde{D}_{n}, \ldots, \widetilde{D}_{n}\right)}{\mathscr{H}\left(A ; \widetilde{D}_{n}, \ldots, \widetilde{D}_{n}\right)}=\frac{\operatorname{deg}_{\iota} \mathrm{B}}{\operatorname{deg}_{\iota} A}\left(\frac{1}{\widetilde{D}_{n}}\right)^{g-g_{\mathrm{H}}} .
$$

Les majorations $\widetilde{D}_{0} \leq \widetilde{T} S_{0}^{1-y} / \mathscr{q 1}_{\text {et }}$ et $\widetilde{D}_{n} \leq \widetilde{T} / \emptyset_{15}$ entraînent alors

$$
1=x(\mathrm{H})^{t-t_{\mathrm{H}}} \geq\left(\frac{1}{\widetilde{T}}\right)^{r_{\mathrm{H}}-\lambda_{\mathrm{H}}} \frac{q_{14}^{t_{\mathrm{H}}} c_{15}^{g_{\mathrm{H}}}(S+1) \operatorname{deg}_{\iota} \mathrm{B}}{d_{16}\left(\operatorname{deg}_{\iota} A\right) S_{0}^{(1-y)\left(t-t_{\mathrm{H}}\right)}} .
$$

Or $t-t_{\mathrm{H}}$ et $g-g_{\mathrm{H}}$ sont des entiers $\geq 1$ car ils sont supérieurs à $\varsigma_{\mathrm{H}}$ (voir (49)), qui est $\geq 1$ par hypothèse. De plus $(1-y)\left(t-t_{\mathrm{H}}\right) \in\{0,1\}$ car $y \in\{0,1\}$ et si $y=0$ alors $t=1$ et $t_{\mathrm{H}}=0$. On en déduit

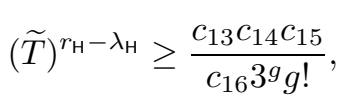

ce qui donne $\widetilde{T} \geq 1$. La majoration (ii) découle directement de la définition de $\widetilde{D}_{0}$ :

$$
\frac{D_{0}}{S_{0}^{1-y}} \leq \frac{\widetilde{D}_{0}}{S_{0}^{1-y}} \leq \frac{U_{0}}{\Phi_{14} S_{0} \log \mathfrak{e}}=\frac{\widetilde{T}}{\mathscr{1 4}_{14}} \leq \frac{2 T}{\delta_{14}} .
$$


Pour $D_{0}>0$, observons que la propriété (5) de $\mathscr{H}$ jointe aux majorations $\widetilde{D}_{i} \leq \widetilde{D}_{n}$, pour $1 \leq i \leq n$, impliquent

$$
\left(x \widetilde{D}_{0}\right)^{t-\widetilde{t}}=\left(x(\widetilde{G}) \widetilde{D}_{0}\right)^{t-\widetilde{t}} \geq\left(\frac{\widetilde{T^{\top}}(S+1)}{\widetilde{D}_{n}^{g-\widetilde{g}}}\right)\left(\frac{\operatorname{deg}_{\iota} \widetilde{A}}{\widetilde{1 \in} \operatorname{deg}_{\iota} A}\right) .
$$

Et comme $\widetilde{T} \geq 19 \widetilde{D}_{n}, \widetilde{T} \geq 1$ et $\widetilde{\lambda} \geq g-\widetilde{g} \geq 1$ (inégalité (49)), nous obtenons

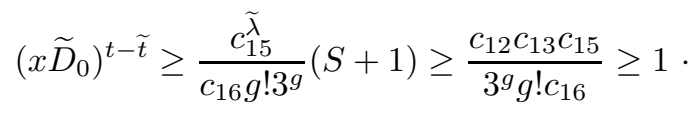

Comme $t \neq \widetilde{t}$ (grâce une fois de plus à la relation (49)), nous déduisons $D_{0} \geq 1$. Par ailleurs, en utilisant l'inégalité $\widetilde{T} \geq 1 \widetilde{D}_{0} / S_{0}^{1-y}$ que nous venons de montrer, ainsi que $\widetilde{T} \geq 1,1 \geq x$ et $\widetilde{\lambda} \geq t-\widetilde{t} \geq 1$, l'inégalité (51) implique

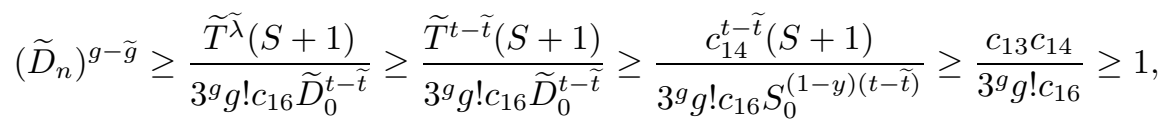

ce qui entraîne $D_{n} \geq 1$ car $g \neq \widetilde{g}$. En ce qui concerne l'estimation (iv), nous avons une majoration de $D_{0}$ grâce à $x \leq x(\{0\})$ :

$$
D_{0} \leq x(\{0\}) \widetilde{D}_{0}=\left(\frac{\widetilde{T}^{g}(S+1)}{c_{\text {国 }} \mathscr{H}\left(A ; \widetilde{D}_{1}, \ldots, \widetilde{D}_{n}\right)}\right)^{1 / t} \leq\left(\left(\frac{\widetilde{T}}{\widetilde{D}_{1}}\right)^{g} \frac{S+1}{3^{g} g ! \Phi_{16}}\right)^{1 / t}
$$

et donc

$$
\begin{aligned}
& 2(g+t) T \log \left(4 D_{0}\right) \\
& \quad \leq \frac{2(g+t) T}{t} \log \left\{\left(\frac{4^{t}(S+1)}{3^{g} g ! \text { 进 }}\right)\left(\text { 国 }\left(1+\frac{D S_{0}}{\log \mathfrak{e}} \log a_{1}\right)\right)^{g}\right\} .
\end{aligned}
$$

Or on a

$$
\begin{aligned}
\log \left(1+\frac{D S_{0}}{\log \mathfrak{e}} \log a_{1}\right) & \leq \log ^{+} \log a_{1}+\log ^{+}\left(\frac{D}{\log \mathfrak{e}}\right)+\log \left(S_{0}+1\right) \\
& \leq \frac{2 \mathfrak{a} \log \mathfrak{e}}{D}+\log \left(S_{0}+1\right)
\end{aligned}
$$

ce qui entraîne

$$
2(g+t) T \log \left(4 D_{0}\right) \leq 2(g+1) T\left\{c_{18}+(g+1) \log \mathfrak{a}+2 g \frac{\mathfrak{a} \log \mathfrak{e}}{D}\right\}
$$

avec

De plus, on a

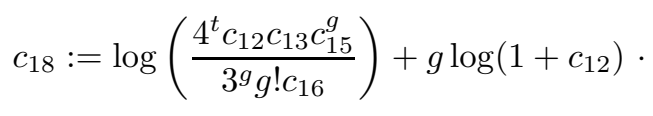

$$
\log \mathfrak{a}=\log \left(\frac{\mathfrak{a} \log \mathfrak{e}}{D} \times \frac{D}{\log \mathfrak{e}}\right) \leq \log ^{+}\left(\frac{D}{\log \mathfrak{e}}\right)+\frac{\mathfrak{a} \log \mathfrak{e}}{D} \leq \frac{2 \mathfrak{a} \log \mathfrak{e}}{D}
$$

donc

$$
\begin{aligned}
2(g+t) T \log \left(4 D_{0}\right) & \leq 2(g+1) T\left(\text { 国 }+4(g+2) \frac{\mathfrak{a} \log \mathfrak{e}}{D}\right) \\
& \leq 2(g+1)(\text { 国 }+4 g+2) \widetilde{T} \frac{\mathfrak{a} \log \mathfrak{e}}{D} \leq \text { 迎 } \frac{U_{0}}{D}
\end{aligned}
$$

avec

$$
c_{19}:=\frac{2(g+1)(4 g+2+d+19)}{\text { c⿴囗十 }} \text {. }
$$

Pour montrer que 500 1 过 $\leq 1$, observons que

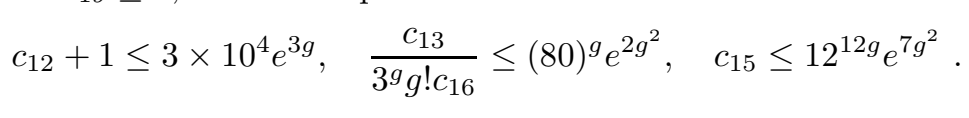


Ces inégalités — assez larges — sont obtenues avec les estimations $t \leq g, n \leq g, g ! \leq g^{g-1}, x \leq e^{x / 2}$ pour tout $x \geq 0$ et la définition des constantes $c_{i}$ de la manière suivante :

$$
\begin{gathered}
\text { 12 }+1=(10 g)^{4}(g+t) n+1 \leq(10 g)^{4}(g+g) g+1 \leq 3 \times 10^{4} g^{6} \leq 3 \times 10^{4} e^{3 g}, \\
\frac{113}{3^{g} g ! c_{16}}=2^{g+t} t !(2(g+t)+1)^{g}(g+t)^{2} \\
\leq 2^{2 g} g !(5 g)^{g}(2 g)^{2} \leq(80)^{g} g^{2 g+1} \leq(80)^{g} e^{2 g^{2}} .
\end{gathered}
$$

Et enfin, comme 1 1 $\leq 12^{g} \times 2^{g+t} g ! \leq 12^{g} \times 2^{2 g} \times g^{g-1}$, on a

$$
\begin{aligned}
{[13} & \leq 3^{g} g !\left(12^{g} 2^{2 g} g^{g-1}\right) \times(80)^{g} e^{2 g^{2}} \\
& \leq 2^{4 g} 3^{2 g} g^{2 g-2} \times(80)^{g} e^{2 g^{2}} .
\end{aligned}
$$

En reportant cette estimation dans $\phi_{15}=100(48 \pi+6)(g+t) n q_{13}^{2}$ on a

$$
10 \leq\left(200(48 \pi+6) \times 2^{8} \times 3^{4} \times 80\right)^{g} g^{4 g-2} e^{4 g^{2}} \leq(12)^{12 g} e^{7 g^{2}} .
$$

Nous constatons alors que $c$ 国 $\leq 72 g^{3}$ puis $50 q 19 \leq 1$ car

$$
\not 19 \leq \frac{2(g+1)\left(4 g+2+72 g^{3}\right)}{(10 g)^{4}(g+t) n} \leq \frac{2\left(4 g+2+72 g^{3}\right)}{(10 g)^{4}} \leq \frac{1}{50} .
$$

Ce qui démontre l'inégalité (iv). La majoration (v) découle immédiatement de $D_{i} \leq \widetilde{D}_{i}$ et du choix des paramètres. Quant à la dernière estimation, elle est une conséquence de $\widetilde{D}_{n} \geq 1$ et $\log \mathfrak{e} \geq 1$ :

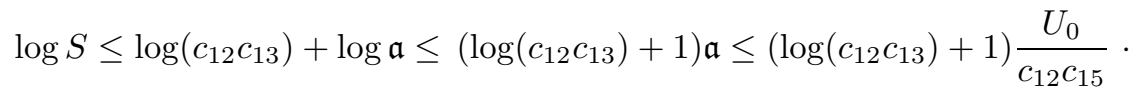

Nous pouvons maintenant formuler l'énoncé qui généralise le théorème 3.1.

Théorème 5.2. Avec les notations ci-dessus et l'hypothèse sur p énoncée dans le paragraphe 3.2, il existe une constante (totalement explicite) $c_{20}>0$, qui ne dépend que de $g$, $t$ et du sous-groupe $\mathrm{H}$, telle que

$$
\log \mathrm{d}\left(u, W_{\mathbf{C}}\right) \geq-\phi 20
$$

On peut choisir $\left[2024 g^{2}(g+t)\right.$.

Nous renvoyons à la fin de la preuve le lien qui unit cet énoncé au théorème 3.1. Ainsi, nous nous concentrons dorénavant sur la preuve de l'inégalité (52). Le premier pas est un lemme d'injectivité qui est l'objet du paragraphe suivant.

5.4. Lemme de multiplicités. Commençons par introduire quelques notations supplémentaires. D'après le lemme 5.1, (iii), nous pouvons définir le nombre $\kappa$ comme le plus petit entier compris entre 1 et $n$ tel que $D_{\kappa} \geq 1$. Notons $\pi_{\kappa}: A \rightarrow A_{\kappa} \times \cdots \times A_{n}$ la projection canonique (ce n'est pas la même application que celle définie dans la propriété (3) de $\mathscr{H}$, mais cela ne devrait créer aucune confusion dans la suite). Notons également $M$ le fibré en droites

$$
\mathcal{O}_{X_{0}}\left(D_{0}\right) \otimes L_{1}^{\otimes 3 D_{1}} \otimes \cdots \otimes L_{n}^{\otimes 3 D_{n}}
$$

au-dessus de l'adhérence schématique $X$ de $\iota(G)$ dans $\mathbf{P}\left(\right.$ pour $1 \leq i \leq \kappa-1, L_{i}^{\otimes 3 D_{i}}=\mathcal{O}_{A_{i}}$ ).

Proposition 5.3. Aucun élément non nul $s \in \mathrm{H}^{0}(X, M)$ ne s'annule le long de $W$ à l'ordre $(g+t) T$ en tous les points de $\left\{0_{G}, p, 2 p, \ldots,(g+t) S p\right\}$.

La démonstration de cette proposition s'appuie sur le « nouveau » lemme de multiplicités de Philippon [56]. Pour simplifier nous employons nos notations qui sont à prendre ici en un sens «élargi » (par exemple pour cet énoncé, $W$ est un sous-espace quelconque de $\left.t_{G}\right)$.

Lemme 5.4 (Philippon). Soit $\Sigma$ un sous-ensemble fini de points de $G(\bar{k})$ contenant l'origine et notons, pour $\alpha \in \mathbf{N} \backslash\{0\}, \Sigma(\alpha):=\left\{x_{1}+\cdots+x_{\alpha} ; x_{i} \in \Sigma\right\}$. Supposons qu'il existe un polynôme 
multihomogène de $\mathbf{P}_{\bar{k}}$ de multidegré $\leq\left(D_{0}, \ldots, D_{n}\right)$, non identiquement nul sur $G_{\sigma_{0}}(\mathbf{C})$, qui s'annule le long de $W$ à l'ordre $(g+t) T$ en chaque point de $\Sigma(g+t)$. Alors il existe un sous-groupe algébrique connexe $G^{\star}$ de $G_{\bar{k}}$, distinct de $G_{\bar{k}}$, tel que

$$
\begin{aligned}
& T^{\operatorname{codim}_{W_{\bar{k}}}\left(W_{\bar{k}} \cap t_{G^{\star}}\right)} \operatorname{card}\left(\frac{\Sigma+G^{\star}(\bar{k})}{G^{\star}(\bar{k})}\right) \mathscr{H}\left(G^{\star} ; D_{0}, \ldots, D_{n}\right) \\
& \leq 2^{g} \mathscr{H}\left(G ; D_{0}, \ldots, D_{n}\right) .
\end{aligned}
$$

Cet énoncé s'obtient à partir du théorème 1 de [56] appliqué avec un plongement de SegreVeronese de $\mathbf{P}_{\bar{k}}$ dans un espace projectif $\mathbf{P}_{\bar{k}}^{N}$, voir $\S 7$, ibid. Notons au passage qu'un résultat de Lange 43. permet de prendre $c=2$ dans le théorème 1 en question car $A_{i, \sigma_{0}}$ est projectivement normal dans $\mathbf{P}_{\mathbf{C}}^{N_{i}}$. Comparé avec le précédent lemme de multiplicités du même auteur, il permet de supprimer un coefficient $g$ ! dans le membre de droite de (54). L'énoncé précis que nous avons écrit ci-dessus est quant à lui extrait (sous une forme particulière) de l'article [67 de Waldschmidt (proposition 3.1). En utilisant ce lemme avec $\Sigma=\Sigma_{p}(S):=\{0, p, \ldots, S p\}$, nous allons pouvoir démontrer la proposition 5.3 .

Démonstration de la proposition 5.9. Supposons qu'il existe une telle section $s \neq 0$. Pour chaque entier $1 \leq i \leq n$, le fibré en droites complexe $L_{i, \sigma_{0}}^{\otimes 3}$ sur $A_{i, \sigma_{0}}(\mathbf{C})$ est normalement engendré et l'application de restriction

$$
\mathrm{H}^{0}\left(\mathbf{P}_{\mathbf{C}}^{N_{i}}, \mathcal{O}\left(D_{i}^{\prime}\right)\right) \rightarrow \mathrm{H}^{0}\left(A_{i, \sigma_{0}}(\mathbf{C}), L_{i, \sigma_{0}}^{\otimes 3 D_{i}^{\prime}}\right)
$$

(où $N_{i}:=\mathrm{h}^{0}\left(A_{i}, L_{i}^{\otimes 3}\right)-1=3^{g_{i}}-1$ ) est surjective (voir [5], chap. 7, théorème (3.1)). Par conséquent, la section $s$ provient d'un polynôme $P\left(\mathbf{X}_{0}, \ldots, \mathbf{X}_{n}\right)$, homogène en les variables $\mathbf{X}_{i}=$ $\left(X_{i, 0}, \ldots, X_{i, N_{i}}\right)$, à coefficients complexes et de degré partiel relatif à $\mathbf{X}_{i}$ inférieur à $D_{i}^{\prime}$. La propriété d'annulation de $s$ se traduit par une propriété similaire pour $P$, ce qui permet d'utiliser le lemme 5.4. De la sorte, il existe un sous-groupe algébrique connexe et strict $G^{\star} \operatorname{de} G$ tel que

$$
\begin{aligned}
& T^{\operatorname{codim}_{W_{\bar{k}}}\left(W_{\bar{k}} \cap t_{G^{\star}}\right)} \operatorname{card}\left(\frac{\Sigma_{p}(S)+G^{\star}(\bar{k})}{G^{\star}(\bar{k})}\right) \mathscr{H}\left(G^{\star} ; D_{0}^{\prime}, \ldots, D_{n}^{\prime}\right) \\
& \leq 2^{g} \mathscr{H}\left(G ; D_{0}^{\prime}, \ldots, D_{n}^{\prime}\right) .
\end{aligned}
$$

Montrons que cette inégalité ne peut jamais être satisfaite. Pour cela, nous allons distinguer plusieurs cas selon $G^{\star}$. Décomposons $G^{\star}$ en $G_{0}^{\star} \times A^{\star}$ avec $G_{0}^{\star} \subseteq G_{0}$ et $A^{\star} \subseteq A$ (ici le signe « $\subseteq$ signifie «sous-groupe algébrique connexe de 》) et considérons les entiers $t^{\star}, g^{\star}, d^{\star}, r^{\star}, \lambda^{\star}$ associés à $G^{\star}$ par les formules (48).

Tout d'abord, supposons que $t_{G^{\star}}+W_{\bar{k}}=t_{G_{\bar{k}}}$. Alors $r^{\star}=\lambda^{\star}$ et l'inégalité (55) entraîne

$$
\begin{aligned}
T^{r^{\star}} \operatorname{card}\left(\frac{\Sigma_{p}(S)+G^{\star}(\bar{k})}{G^{\star}(\bar{k})}\right)\left(\begin{array}{c}
d^{\star} \\
t^{\star}
\end{array}\right) & \leq 2^{g}\left(\begin{array}{c}
g+t \\
t
\end{array}\right) D_{0}^{t-t^{\star}} \frac{\mathscr{H}\left(A ; D_{1}^{\prime}, \ldots, D_{n}^{\prime}\right)}{\mathscr{H}\left(A^{\star} ; D_{1}^{\prime}, \ldots, D_{n}^{\prime}\right)} \\
& \leq 6^{g} \frac{(g+t) !}{t !} D_{0}^{t-t^{\star}} D_{n}^{g-g^{\star}}
\end{aligned}
$$

via la propriété $(5)$ de $\mathscr{H}$ et les majorations $D_{i}^{\prime} \leq D_{n}$, valides pour $1 \leq i \leq n$. Lorsque $y=1$, nous minorons card $\left(\frac{\Sigma_{p}(S)+G^{\star}(\bar{k})}{G^{\star}(k)}\right)$ par 1 et la majoration (56) implique

$$
T^{r^{\star}} \leq c_{21} \max \left\{D_{0}, D_{n}\right\}^{r^{\star}}
$$

avec $021:=6^{g}(g+t) !(t !)^{-1}\left(\begin{array}{c}d^{\star} \\ t^{\star}\end{array}\right)$, ce qui contredit le lemme 5.1. (i) et (ii) (car $G^{\star} \neq G_{\bar{k}}$ et $215>21$ et $\left.h_{14}>22_{21}\right)$. Lorsque $y=0$, le sous-espace $W_{0}$ est par définition un hyperplan $(t=1)$, donc la dimension de $G_{0}$ est 1 et $G_{0}^{\star}=\{0\}$ ou $G_{0, \bar{k}}$ (le groupe $G_{0}^{\star}$ étant connexe). Si $G_{0}^{\star}=\{0\}$ alors

$$
\operatorname{card}\left(\frac{\Sigma_{p}(S)+G^{\star}(\bar{k})}{G^{\star}(\bar{k})}\right)=S+1 \quad \text { (Hypothèse (2) sur le point } p, \text { p. 9) }
$$

et l'inégalité (56) conduit à la majoration

$$
T(S+1) \leq \frac{\phi_{21}}{\left.\phi_{15}\right|^{g^{\star}}} D_{0} \leq{ }_{21} D_{0}
$$

au moyen de $T \geq 1 D_{15} D_{n}$ (lemme 5.1, (i)) et $r^{\star}=1+g-g^{\star}$. Il y a alors une contradiction avec le lemme 5.1, (ii), car $113614>24$. Si $G_{0}^{\star}=G_{0, \bar{k}}$ alors (56) implique $T^{g-g^{\star}} \leq \phi_{21} D_{n}^{g-g^{\star}}$. Comme 
$g^{\star} \neq g\left(\right.$ sinon $\left.G^{\star}=G_{\bar{k}}\right)$, cette inégalité contredit le lemme 5.1, (i), car $q_{15}>$ 电. Cela démontre que $\lambda^{\star}<r^{\star}$ i.e. $t_{G^{\star}}+W_{\bar{k}} \neq t_{G_{\bar{k}}}$. En particulier, nous avons

$$
\operatorname{card}\left(\frac{\Sigma_{p}(S)+G^{\star}(\bar{k})}{G^{\star}(\bar{k})}\right)=S+1
$$

à cause de l'hypothèse (1) sur $p$. Définissons alors les sous-groupes algébriques auxiliaires suivants. Soit

$$
A_{\kappa}^{\star}:=A_{1} \times \cdots \times A_{\kappa-1} \times \pi_{\kappa}\left(A^{\star}\right) \quad \text { et } \quad G_{\kappa}^{\star}:=G_{0}^{\star} \times A_{\kappa}^{\star} \quad(\text { définis sur } \bar{k}) \text {. }
$$

Un calcul direct donne

$$
\mathscr{H}\left(G_{\kappa}^{\star} ; D_{0}^{\prime}, \ldots, D_{n}^{\prime}\right)=c_{22} D_{0}^{t^{\star}} \mathscr{H}\left(\pi_{\kappa}\left(A^{\star}\right) ; D_{\kappa}, \ldots, D_{n}\right)
$$

avec

$$
\text { 吗 }:=\frac{\left(\operatorname{dim} G_{\kappa}^{\star}\right) ! 3^{g_{1}+\cdots+g_{\kappa-1}}}{t^{\star} !\left(\operatorname{dim} \pi_{\kappa}\left(A^{\star}\right)\right) !} .
$$

Observons par ailleurs que la propriété (3) de $\mathscr{H}$ implique

$$
\left(\begin{array}{c}
g^{\star} \\
\operatorname{dim} \pi_{\kappa}\left(A^{\star}\right)
\end{array}\right) \mathscr{H}\left(\pi_{\kappa}\left(A^{\star}\right) ; D_{\kappa}, \ldots, D_{n}\right) \leq \mathscr{H}\left(A^{\star} ; D_{1}^{\prime}, \ldots, D_{n}^{\prime}\right)
$$

puis

$$
\mathscr{H}\left(G_{\kappa}^{\star} ; D_{0}^{\prime}, \ldots, D_{n}^{\prime}\right) \leq c_{23} \mathscr{H}\left(G^{\star} ; D_{0}^{\prime}, \ldots, D_{n}^{\prime}\right)
$$

où

$$
\begin{aligned}
& c_{23}:=\text { 电2 }^{t^{\star} !\left(\operatorname{dim} \pi_{\kappa}\left(A^{\star}\right)\right) !\left(g^{\star}-\operatorname{dim} \pi_{\kappa}\left(A^{\star}\right)\right) !} \\
& =\frac{\left(\operatorname{dim} G_{\kappa}^{\star}\right) ! 3^{g_{1}+\cdots+g_{\kappa-1}}\left(g^{\star}-\operatorname{dim} \pi_{\kappa}\left(A^{\star}\right)\right) !}{d^{\star} !} .
\end{aligned}
$$

Montrons que $G_{\kappa^{*}}^{\star} \neq G_{\bar{k}}$. Dans le cas contraire, nous avons $\lambda^{\star}=\operatorname{codim}_{W_{\bar{k}}}\left(W_{\bar{k}} \cap t_{G^{\star}}\right)=0$ et des relations (55) et (58) nous déduisons la majoration

$$
(S+1) \mathscr{H}\left(G^{\star} ; D_{0}^{\prime}, \ldots, D_{n}^{\prime}\right) \leq 2^{g} \mathscr{H}\left(G ; D_{0}^{\prime}, \ldots, D_{n}^{\prime}\right)
$$

Avec l'inégalité (59), nous obtenons $S+1 \leq 2^{g} c_{23}$, ce qui contredit

$$
\text { 吅直 } \geq 6^{g} \frac{(g+t) !}{t !}>2^{g} c_{23} \text {. }
$$

Nous affirmons maintenant que $t_{G_{k}^{\star}}+W_{\bar{k}} \neq t_{G_{\bar{k}}}$. En effet, dans le cas contraire, nous avons

$$
\operatorname{codim}_{G_{\bar{k}}} G_{\kappa}^{\star}=\operatorname{codim}_{W_{\bar{k}}}\left(W_{\bar{k}} \cap t_{G_{\kappa}^{\star}}\right)
$$

et, puisque

$$
\operatorname{codim}_{W_{\bar{k}}}\left(W_{\bar{k}} \cap t_{G_{\bar{k}}^{\star}}\right) \leq \operatorname{codim}_{W_{\bar{k}}}\left(W_{\bar{k}} \cap t_{G^{\star}}\right), \quad \operatorname{card}\left(\frac{\Sigma_{p}(S)+G_{\kappa}^{\star}(\bar{k})}{G_{\kappa}^{\star}(\bar{k})}\right) \leq S+1,
$$

les inégalités (55) et (59) entraînent

$$
\begin{aligned}
& T^{\operatorname{codim}_{W_{\bar{k}}}\left(W_{\bar{k}} \cap t_{G_{\kappa}^{\star}}\right)} \operatorname{card}\left(\frac{\Sigma_{p}(S)+G_{\kappa}^{\star}(\bar{k})}{G_{\kappa}^{\star}(\bar{k})}\right) \mathscr{H}\left(G_{\kappa}^{\star} ; D_{0}^{\prime}, \ldots, D_{n}^{\prime}\right) \\
& \leq 2^{g} c_{23} \mathscr{H}\left(G ; D_{0}^{\prime}, \ldots, D_{n}^{\prime}\right) .
\end{aligned}
$$

Nous pouvons alors reprendre le même argument que celui utilisé pour montrer que $t_{G^{\star}}+W_{\bar{k}} \neq t_{G_{\bar{k}}}$ dans la première partie de la preuve. Il suffit de changer la constante $2^{g}$ qui est dans (55) par $2^{g} c_{23}$ (et donc Q $11_{1}$ est remplacé par le produit $\phi_{21} c_{23}$ ) et la même démarche que ci-dessus entraîne une contradiction avec les points (i) et (ii) du lemme 5.1 car, comme

$$
\text { 21] } c_{23}<18^{g}\left(\frac{(g+t) !}{t !}\right)^{2}
$$

(évident à partir de l'estimation (60) de $c_{23}$ et de la définition de 20 ) nous avons

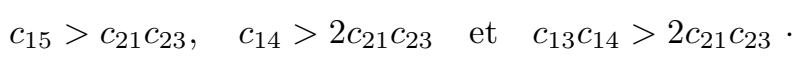


Cela démontre que $t_{G_{\kappa}^{\star}}+W_{\bar{k}} \neq t_{G_{\bar{k}}}$. En remarquant alors que l'égalité (58) reste vraie pour $G_{\kappa}^{\star}$ grâce à l'hypothèse (1) sur $p$, nous allons obtenir une contradiction à partir de (61). En effet, le quotient

$$
\frac{\mathscr{H}\left(G ; D_{0}^{\prime}, \ldots, D_{n}^{\prime}\right)}{\mathscr{H}\left(G_{\kappa}^{\star} ; D_{0}^{\prime}, \ldots, D_{n}^{\prime}\right)}=c_{24} D_{0}^{t-t^{\star}} \frac{\mathscr{H}\left(\pi_{\kappa}(A) ; D_{\kappa}, \ldots, D_{n}\right)}{\mathscr{H}\left(\pi_{\kappa}\left(A^{\star}\right) ; D_{\kappa}, \ldots, D_{n}\right)}
$$

avec

$$
\text { 24 }:=\frac{(g+t) ! t^{\star} !\left(\operatorname{dim} \pi_{\kappa}\left(A^{\star}\right)\right) !}{t !\left(\operatorname{dim} \pi_{\kappa}(A)\right) !\left(\operatorname{dim} G_{\kappa}^{\star}\right) !}
$$

est majoré par

$$
\left[24\left(x \widetilde{D}_{0}\right)^{t-t^{\star}} \frac{\mathscr{H}\left(\pi_{\kappa}(A) ; \widetilde{D}_{\kappa}, \ldots, \widetilde{D}_{n}\right)}{\mathscr{H}\left(\pi_{\kappa}\left(A^{\star}\right) ; \widetilde{D}_{\kappa}, \ldots, \widetilde{D}_{n}\right)}\right.
$$

par définition de $D_{0}=\left[x \widetilde{D}_{0}\right]$ et en vertu de la propriété $(5)$ de $\mathscr{H}$ utilisée conjointement avec $D_{i} \leq \widetilde{D}_{i}$ pour $1 \leq i \leq n$. Cette dernière expression est elle-même égale à

$$
c_{25}\left(x \widetilde{D}_{0}\right)^{t-t^{\star}} \frac{\mathscr{H}\left(A ; \widetilde{D}_{1}, \ldots, \widetilde{D}_{n}\right)}{\mathscr{H}\left(A_{\kappa}^{\star} ; \widetilde{D}_{1}, \ldots, \widetilde{D}_{n}\right)}
$$

avec

$$
c_{25}:=\frac{\left(\operatorname{dim} A_{\kappa}^{\star}\right) !\left(\operatorname{dim} \pi_{\kappa}(A)\right) !}{g !\left(\operatorname{dim} \pi_{\kappa}\left(A^{\star}\right)\right) !} c_{24}=\frac{(g+t) ! t^{\star} !\left(\operatorname{dim} A_{\kappa}^{\star}\right) !}{\left(\operatorname{dim} G_{\kappa}^{\star}\right) ! t ! g !} .
$$

Ce dernier majorant se récrit à l'aide de $x\left(G_{\kappa}^{\star}\right)$ car, par définition de ce nombre réel, on a

$$
\left(x\left(G_{\kappa}^{\star}\right) \widetilde{D}_{0}\right)^{t-\tilde{t}}=(\widetilde{T})^{\operatorname{codim}_{W_{\bar{k}}}\left(W_{\bar{k}} \cap t_{G_{\kappa}^{\star}}\right)}(S+1) \times \frac{\mathscr{H}\left(A_{\kappa}^{\star} ; \widetilde{D}_{1}, \ldots, \widetilde{D}_{n}\right)}{d_{16} \mathscr{H}\left(A ; \widetilde{D}_{1}, \ldots, \widetilde{D}_{n}\right)} .
$$

Compte tenu de $T \geq \widetilde{T} / 2$, l'inégalité 61) implique :

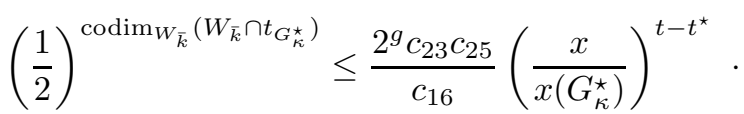

Ceci est impossible car $\longdiv { 1 6 } > 4 ^ { g } c _ { 2 3 } c _ { 2 5 }$ (ce dernier produit doit être évalué à partir de la définition des constantes et non avec les majorations de celles-ci données au cours de la preuve).

Finalement, le sous-groupe $G^{\star}$ ne peut exister dans aucun cas et la proposition 5.3 est démontrée.

5.5. Description du premier fibré hermitien. Dans ce paragraphe, nous définissons le fibré vectoriel hermitien qui est le point de départ de la construction de tous les objets nécessaires pour mettre en œuvre la méthode des pentes.

Pour chaque entier $i \in\{1, \ldots, n\}$, considérons un modèle de Moret-Bailly

$$
\left(\pi_{i}: \mathcal{A}_{i} \rightarrow \operatorname{Spec}\left(\mathcal{O}_{K}\right), \overline{\mathcal{L}}_{i},\left\{\varepsilon_{m p_{i}}: \operatorname{Spec}\left(\mathcal{O}_{K}\right) \rightarrow \mathcal{A}_{i}\right\}_{m \in\{0, \ldots,(g+t) S\}}\right)
$$

de $\left(A_{i}, L_{i}, \Sigma_{p_{i}}((g+t) S)\right)$ sur une extension finie $K$ de $k$ (au sens du $\S 4.3 .2$ ).

Soit $\mathcal{A}:=\mathcal{A}_{1} \times \cdots \times \mathcal{A}_{n}$ et $\mathcal{W}_{0}$ le $\mathcal{O}_{K}$-module saturé $t_{\mathcal{A}} \cap\left(W_{0} \otimes_{k} K\right)$. Soit $\mathscr{G}$ le schéma en groupes lisse $\mathbf{V}\left(\left(t_{\mathcal{A}} / \mathcal{W}_{0}\right)^{\mathrm{v}}\right) \times \mathcal{A} \operatorname{sur} \operatorname{Spec}\left(\mathcal{O}_{K}\right)$, de fibre générique $G_{K}$. Notons $\mathcal{X}$ le schéma $\mathbf{P}\left(\mathcal{O}_{K} \oplus\left(t_{\mathcal{A}} / \mathcal{W}_{0}\right)^{\vee}\right) \times \mathcal{A} \operatorname{sur} \operatorname{Spec}\left(\mathcal{O}_{K}\right)$, et, pour simplifier l'écriture de quelques expressions ultérieures, posons $\mathcal{X}_{0}:=\mathbf{P}\left(\mathcal{O}_{K} \oplus\left(t_{\mathcal{A}} / \mathcal{W}_{0}\right)^{\mathrm{v}}\right)$. Sa fibre générique $\mathcal{X}_{K}$ s'identifie à $X_{K}$, défini avant la proposition 5.3. Les points $k$-rationnels $m p \in G(k), 0 \leq m \leq(g+t) S$, se prolongent en des sections $\varepsilon_{m p} \in \mathcal{X}\left(\mathcal{O}_{K}\right)$, grâce aux $\left(\varepsilon_{m p_{i}}\right)_{1 \leq i \leq n}$. Enfin, considérons $\mathcal{W}$ le graphe de la projection canonique $\lambda: t_{\mathcal{A}} \rightarrow t_{\mathcal{A}} / \mathcal{W}_{0}$ c'est-à-dire le sous- $\mathcal{O}_{K}$-module de $t_{\mathscr{G}}=\left(t_{\mathcal{A}} / \mathcal{W}_{0}\right) \oplus t_{\mathcal{A}}$ composé des éléments $\lambda(z) \oplus z, z \in t_{\mathcal{A}}$. Sa fibre générique $W_{K}=\mathcal{W} \otimes K$ est l'espace vectoriel de $t_{G}(K)$ (de dimension $g$ ) considéré au $\S 3.1$ (après extension des scalaires). Sur le schéma $\mathcal{X}$, nous pouvons définir le faisceau inversible

$$
\mathcal{M}:=\mathcal{O}_{\mathcal{X}_{0}}\left(D_{0}\right) \otimes \mathcal{L}_{1}^{\otimes 3 D_{1}} \otimes \cdots \otimes \mathcal{L}_{n}^{\otimes 3 D_{n}}
$$

Il est muni de métriques hermitiennes aux places archimédiennes de $K$, obtenues par somme hermitiennes des métriques cubistes sur chacun des $\overline{\mathcal{L}_{i}}$ et des métriques de Fubini-Study sur $\overline{\mathcal{O}_{\mathcal{X}_{0}}(1)}$. 
Le $\mathcal{O}_{K}$-module $\mathcal{E}:=\mathrm{H}^{0}(\mathcal{X}, \mathcal{M})$ des section globales de $\mathcal{M}$ est localement libre de type fini. Son rang est

$$
\operatorname{rg} \mathcal{E}=\left(\begin{array}{c}
D_{0}+t \\
t
\end{array}\right) \prod_{i=1}^{n} \mathrm{~h}^{0}\left(A_{i}, L_{i}^{\otimes 3 D_{i}}\right)=\left(\begin{array}{c}
D_{0}+t \\
t
\end{array}\right)\left(3 D_{\kappa}\right)^{g_{\kappa}} \cdots\left(3 D_{n}\right)^{g_{n}}
$$

(nous avons utilisé ici au passage l'amplitude des $L_{i}$ ). Étant donné un plongement complexe $\sigma$ : $K \hookrightarrow \mathbf{C}$, le $\mathbf{C}$-espace vectoriel $\mathcal{E}_{\sigma}:=\mathcal{E} \otimes_{\sigma} \mathbf{C}$ est muni d'une métrique hermitienne $\|\cdot\|_{\overline{\mathcal{E}}, \sigma}$ définie par

$$
\forall s \in \mathcal{E}_{\sigma}, \quad\|s\|_{\mathcal{E}, \sigma}^{2}:=\int_{\mathcal{X}_{\sigma}(\mathbf{C})}\|s(x)\|_{\bar{x}^{*} \mathcal{M}, \sigma}^{2} \mathrm{~d} \mu_{\sigma}(x)
$$

où $\mathrm{d} \mu_{\sigma}$ est la mesure de probabilité produit sur l'ensemble compact $\mathcal{X}_{\sigma}(\mathbf{C})$, obtenue à partir de la mesure $\mathrm{d} \mu_{\mathrm{FS}}$ sur $\mathcal{X}_{0, \sigma}(\mathbf{C})$, invariante sous l'action du groupe unitaire $\mathbf{U}_{t+1}(\mathbf{C})$, et de la mesure de Haar de $A_{\sigma}(\mathbf{C})$ (voir $\S$ 4.6). Le fibré vectoriel hermitien $\overline{\mathcal{E}}=\left(\mathcal{E},\left(\|\cdot\|_{\overline{\mathcal{E}}, \sigma}\right)_{\sigma}\right)$ construit de cette manière possède une pente d'Arakelov $\widehat{\mu}(\overline{\mathcal{E}})$. La proposition suivante est le calcul explicite de cette quantité.

Proposition 5.5. La pente d'Arakelov normalisée de $\overline{\mathcal{E}}$ est

$$
\begin{aligned}
\widehat{\mu}(\overline{\mathcal{E}})=\frac{1}{2} \log \left\{\left(\begin{array}{c}
D_{0}+t \\
t
\end{array}\right) \gamma_{t, D_{0}}\right\}-\frac{1}{t+1} D_{0} \check{h}\left(W_{0}\right) \\
+\sum_{i=\kappa}^{n}\left\{-\frac{1}{2} h_{F}\left(A_{i}\right)+\frac{g_{i}}{4} \log \left(\frac{3 D_{i}}{2 \pi}\right)\right\}
\end{aligned}
$$

où $\gamma_{t, D_{0}}$ est défini dans la proposition 4.9. En particulier, avec la notation $\Xi$ du $\S$ 4.9, nous avons

$$
\widehat{\mu}(\overline{\mathcal{E}})-\Xi\left[\left(\mathcal{X}, \overline{\mathcal{M}},\left(\mathrm{d} \mu_{\sigma}\right)_{\sigma: K \hookrightarrow \mathbf{C})}\right) \geq-\frac{U_{0}}{1000 D} .\right.
$$

Démonstration. La définition de $\overline{\mathcal{E}}$ et la propriété de «morphisme de groupes » de la pente d'Arakelov impliquent l'égalité

$$
\widehat{\mu}(\overline{\mathcal{E}})=\widehat{\mu}\left(\overline{\mathrm{H}^{0}\left(\mathcal{X}_{0}, \mathcal{O}_{\mathcal{X}_{0}}\left(D_{0}\right)\right)}\right)+\sum_{i=1}^{n} \widehat{\mu}\left(\overline{\mathrm{H}^{0}\left(\mathcal{A}_{i}, \mathcal{L}_{i}^{\otimes 3 D_{i}}\right)}\right)
$$

L'égalité (65) s'obtient alors directement à partir des calculs de ces pentes donnés au $\S 4.3$. Quant à l'inégalité (66), utilisons tout d'abord les lemmes 4.15 et 4.16 afin d'obtenir une majoration de $\Xi\left[\left(\mathcal{X}, \overline{\mathcal{M}},\left(\mathrm{d} \mu_{\sigma}\right)_{\sigma: K \hookrightarrow \mathbf{C}}\right)\right]:$

$$
\begin{aligned}
& \Xi\left[\left(\mathcal{X}, \overline{\mathcal{M}},\left(\mathrm{d} \mu_{\sigma}\right)_{\sigma: K \hookrightarrow \mathbf{C}}\right)\right] \\
& =\Xi\left[\left(\mathcal{X}_{0}, \overline{\mathcal{O}_{\mathcal{X}_{0}}\left(D_{0}\right)}, \mathrm{d} \mu_{\mathrm{FS}}\right)\right]+\sum_{i=1}^{n} \Xi\left[\left(\mathcal{A}_{i}, \overline{\mathcal{L}_{i}^{\otimes 3 D_{i}}}, \mathrm{~d} \mu_{\text {Haar }}\right)\right] \\
& \leq \frac{1}{2} \log \left(\begin{array}{c}
D_{0}+t \\
t
\end{array}\right)+\sum_{i=1}^{n} \mathrm{c}_{0}\left(g_{i}\right) \max \left\{1, \log ^{+}\left(h_{F}\left(A_{i}\right)\right), \log ^{+}\left(3 D_{i}\right)\right\} .
\end{aligned}
$$


Grâce aux formules (64) et (65), nous obtenons

$$
\begin{aligned}
\widehat{\mu}(\overline{\mathcal{E}}) & -\Xi\left[\left(\mathcal{X}, \overline{\mathcal{M}},\left(\mathrm{d} \mu_{\sigma}\right)_{\sigma: K \hookrightarrow \mathbf{C}}\right)\right] \\
\geq & -\frac{D_{0} \check{h}\left(W_{0}\right)}{t+1}+\sum_{i=\kappa}^{n}\left(-\frac{1}{2} h_{F}\left(A_{i}\right)+\frac{g_{i}}{4} \log \left(\frac{3 D_{i}}{2 \pi}\right)\right) \\
& -\sum_{i=1}^{n} \mathrm{c}_{0}\left(g_{i}\right) \max \left\{1, \log ^{+}\left(h_{F}\left(A_{i}\right)\right), \log ^{+}\left(3 D_{i}\right)\right\} \\
\geq & -\left(\frac{\log (b)}{(t+1) D}\right) D_{0}-\left(\sum_{i=1}^{n} \mathrm{c}_{0}\left(g_{i}\right)\right) \log \left(3 D_{n}\right) \\
& -\sum_{i=1}^{n}\left(\frac{1}{2}+\mathrm{c}_{0}\left(g_{i}\right)\right) \max \left\{1, h_{F}\left(A_{i}\right)\right\}-\frac{g}{4} \log \frac{2 \pi}{3} \\
\geq & -\left(\frac{1}{(t+1) \phi_{14}}+\frac{19 g^{4}}{c_{12} c \text { 通 }}\right) \frac{U_{0}}{D} .
\end{aligned}
$$

Cette dernière inégalité résulte des majorations $\log \left(3 D_{n}\right) \leq 3 D_{n}, 1 \leq D_{n} \leq U_{0} /\left(c_{12} q_{15} D\right)$ ainsi que de

Nous avons alors

$$
\mathrm{h}_{F} \leq \frac{\mathfrak{a} \log \mathfrak{e}}{D} \leq \frac{\mathfrak{a} \log \mathfrak{e}}{D} \widetilde{D}_{n} \leq \frac{U_{0}}{D, 12 \cos }
$$

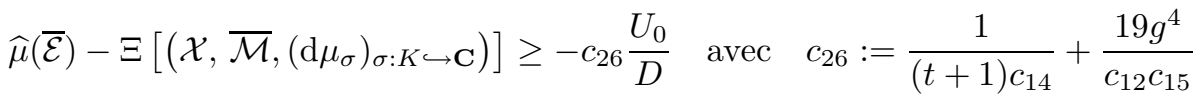

et l'inégalité (66) s'ensuit.

5.6. Choix de l'espace des jets et de sa filtration. Dans ce paragraphe, nous définissons les espaces vectoriels $F, F_{i}, E_{i}, G_{i}$ et les morphismes d'évaluations $\varphi_{i}: E_{i} \rightarrow G_{i}, \varphi: \mathcal{E} \otimes_{\mathcal{O}_{K}} K \rightarrow F$ nécessaires pour employer la méthode des pentes. Avant d'entrer dans le détail de la construction, rappelons la définition de la notion de voisinage infinitésimal d'ordre $\ell$ le long de $W$ au point $q$, noté $V(q, W, \ell)$, introduite par Bost dans 10], $\S 5.2$ et 5.3 (ici $\ell \in \mathbf{N}$ et $q \in G(K)$ ).

- Si $\ell=0, V(q, W, 0)$ est le sous-schéma réduit de $G_{K}$ défini par $q$.

- Si $q=0$ et $\ell=1, V(0, W, 1)$ est le sous-schéma de $G_{K}$ de longueur $g+1$ du voisinage infinitésimal d'ordre 1 en 0 défini par $W$.

- Si $\ell \geq 1, V(q, W, \ell)$ est l'image dans $G_{K}$ du schéma $\{q\} \times V(0, W, 1)^{\ell}$ via le morphisme de multiplication $G_{K}^{\ell+1} \rightarrow G_{K}$,

$$
\left(q, g_{1}, \ldots, g_{\ell}\right) \mapsto q \cdot g_{1} \cdots g_{\ell} .
$$

Dans la suite, nous utiliserons en réalité l'adhérence schématique de $V(q, W, \ell)$ dans $\mathcal{X}_{K}$ en conservant toutefois la même notation. Considérons alors le sous-schéma fermé (non-réduit) de $\mathcal{X}_{K}$ défini par

$$
\mathbf{T}:=\bigsqcup_{m=0}^{S_{0}} V(m p, W, 2(g+t) T) \sqcup \bigsqcup_{m=S_{0}+1}^{(g+t) S} V(m p, W,(g+t) T)
$$

et $F$ le $K$-espace vectoriel des sections de la restriction du fibré en droites $M$ à $\mathbf{T}$ :

$$
F:=\mathrm{H}^{0}\left(\mathbf{T}, M_{\mid \mathbf{T}}\right) \text {. }
$$

Posons $E:=\mathcal{E} \otimes_{\mathcal{O}_{K}} K$ et considérons $\varphi: E \rightarrow F$ le morphisme de restriction.

Lemme 5.6. L'application linéaire $\varphi$ est injective.

Démonstration. Soit $s \in E=\mathrm{H}^{0}(X, M) \otimes_{k} K$ tel que $\varphi(s)=0$. En particulier, la restriction de $s$ au schéma

$$
\bigsqcup_{m=0}^{(g+t) S} V(m p, W,(g+t) T)
$$

s'annule ou, autrement dit, $s$ s'annule à l'ordre $(g+t) T$ le long de $W$ en tous les point de l'ensemble $\{0, p, \ldots,(g+t) S p\}$. D'après la proposition 5.3, on a $s=0$. 
Soit

$$
\nabla:=\left\{(m, \ell) \in \mathbf{N}^{2} ; \quad \begin{array}{c}
0 \leq m \leq S_{0} \text { et } \ell \leq 2(g+t) T \text { ou } \\
S_{0}+1 \leq m \leq(g+t) S \text { et } \ell \leq(g+t) T
\end{array}\right\}
$$

et $N:=\operatorname{card}(\nabla)$. Nous munissons l'ensemble $\nabla$ de l'ordre lexicographique $\preccurlyeq$. Nous pouvons alors ranger les éléments de $\nabla$ en ordre croissant, ce qui permet d'associer à chaque couple $(m, \ell) \in \nabla$ un unique indice $i \in\{1, \ldots, N\}$. Pour un tel entier $i$ associé à $(m, \ell) \in \nabla$, définissons

$$
\mathbf{T}_{i}:=\bigsqcup_{\substack{\left(m^{\prime}, \ell^{\prime}\right) \in \nabla \\\left(m^{\prime}, \ell^{\prime}\right) \preccurlyeq(m, \ell)}} V\left(m^{\prime} p, W, \ell^{\prime}\right) .
$$

Par commodité, posons $\mathbf{T}_{0}:=\emptyset$. Nous avons par exemple $\mathbf{T}_{1}=\{0\}$ et $\mathbf{T}_{N}=\mathbf{T}$. De plus, soit $F_{i}$ le noyau du morphisme de restriction

$$
q_{i}: \mathrm{H}^{0}\left(\mathbf{T}, M_{\mid \mathbf{T}}\right) \rightarrow \mathrm{H}^{0}\left(\mathbf{T}_{i}, M_{\mid \mathbf{T}_{i}}\right) .
$$

Nous obtenons ainsi une filtration de $F$ par des sous-espaces vectoriels (sur $K$ ) :

$$
F=F_{0} \supseteq F_{1} \supseteq \cdots \supseteq F_{N}=\{0\}
$$

dont les quotients intermédiaires $G_{i}:=F_{i-1} / F_{i}$ (pour $1 \leq i \leq N$ ) sont les noyaux des applications $\mathrm{H}^{0}\left(\mathbf{T}_{i}, M_{\mid \mathbf{T}_{i}}\right) \rightarrow \mathrm{H}^{0}\left(\mathbf{T}_{i-1}, M_{\mid \mathbf{T}_{i-1}}\right)$ (lemme du serpent). L'espace vectoriel $G_{i}$ s'identifie à $S^{\ell}\left(W^{\mathrm{v}}\right) \otimes$ $(m p)^{*} M$ où $m p: \operatorname{Spec}(K) \rightarrow \mathcal{X}_{K}$ désigne le point $m p$ de $G(k)$ étendu à $\mathcal{X}_{K}(K)$ par $\left.G_{K} \hookrightarrow \mathcal{X}_{K}\right)$. Pour $i \in\{1, \ldots, N\}$, posons

$$
E_{i}:=\varphi^{-1}\left(F_{i-1}\right) \subseteq E \quad \text { et } \quad \varphi_{i}: E_{i} \rightarrow G_{i}
$$

l'application $K$-linéaire déduite de $\varphi$ et de la projection canonique $F_{i-1} \rightarrow G_{i}$. Pour simplifier l'écriture de l'inégalité de pentes, il est commode également de poser $E_{N+1}:=\varphi^{-1}\left(F_{N}\right)=\{0\}$.

Remarque 5.7. L'application $\varphi_{i}$ peut être décrite de manière moins abstraite. Considérons un voisinage ouvert $U$ de $m p$ dans $\mathcal{X}_{\sigma_{0}}(\mathbf{C})$ tel que $M_{\mid U} \simeq \mathcal{O}_{U}$ et soit $U^{\prime}:=\exp _{\sigma_{0}}^{-1}(U)$ où $\exp _{\sigma_{0}}$ : $t_{G_{\sigma_{0}}}(\mathbf{C}) \rightarrow \mathcal{X}_{\sigma_{0}}(\mathbf{C})$ est l'application exponentielle de $G_{\sigma_{0}}(\mathbf{C})$ prolongée à $\mathcal{X}_{\sigma_{0}}(\mathbf{C})$. Soit $s$ un élément de $E_{i}$. En choisissant un isomorphisme $M_{\mid U} \simeq \mathcal{O}_{U}$, nous pouvons écrire $s(x)=\theta(z) \cdot s_{0}(x)$ avec $x=\exp _{\sigma_{0}}(z), z \in U^{\prime}$ et $\theta$ fonction analytique sur $U^{\prime}$. Par définition, le jet d'ordre $\ell$ le long de $W$ au point $m u$ de $\theta$, noté jet ${ }_{W}^{\ell} \theta(m u)$, est

$$
\sum_{|\mathbf{i}|=\ell} \frac{1}{\mathbf{i} !} \mathrm{D}_{\mathrm{w}}^{\mathbf{i}} \theta(m u) \cdot\left(w_{1}^{*}\right)^{i_{1}} \cdots\left(w_{g}^{*}\right)^{i_{g}} \in S^{\ell}\left(W_{\sigma_{0}}^{\mathrm{v}}\right)
$$

où $\mathbf{w}=\left(w_{1}, \ldots, w_{g}\right)$ est une base de $W$ et $\left(w_{1}^{*}, \ldots, w_{g}^{*}\right)$ sa base duale. On vérifie que cette définition ne dépend pas du choix de w. Alors le jet de $s$ d'ordre $\ell$ le long de $W$ en $m p$ est

$$
\operatorname{jet}_{W}^{\ell} s(m p):=\left(\operatorname{jet}_{W}^{\ell} \theta(m u)\right) \cdot s_{0}(m p) \in S^{\ell}\left(W^{\vee}\right) \otimes(m p)^{*} M \otimes_{\sigma_{0}} \mathbf{C} .
$$

Cela ne dépend pas du choix de la trivialisation de $M_{\mid U}$ (i.e. de l'isomorphisme $M_{\mid U} \simeq \mathcal{O}_{U}$ ) car

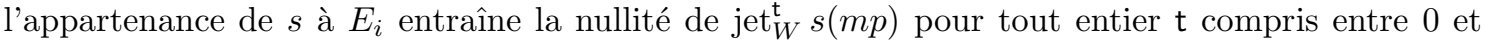
$\ell-1$.

Par ailleurs, l'espace vectoriel $G_{i}$ a une structure entière sous-jacente donnée par le $\mathcal{O}_{K}$-module

$$
\mathcal{G}_{i}:=S^{\ell}\left(\mathcal{W}^{\vee}\right) \otimes \varepsilon_{m p}^{*} \mathcal{M}
$$

Ce module est localement libre et il possède une structure naturelle de fibré vectoriel hermitien $\overline{\mathcal{G}}_{i}$ sur Spec $\mathcal{O}_{K}$. En effet, pour chaque plongement complexe $\sigma: K \hookrightarrow \mathbf{C}$, l'espace tangent $t_{\mathcal{X}_{\sigma}}(\mathbf{C})=$ $\left(t_{A} / W_{0}\right)_{\sigma} \oplus t_{A_{\sigma}}(\mathbf{C})$ peut être muni de la métrique de Riemann sur $t_{A_{\sigma}}(\mathbf{C}$ ) (provenant du fibré en droites $\left.L_{\sigma}\right)$ et de la métrique quotient sur $\left(t_{A} / W_{0}\right)_{\sigma}$. Le morphisme d'inclusion $\mathcal{W} \hookrightarrow t_{\mathcal{X}}$ fournit par restriction une métrique sur $W_{\sigma}$, et donc sur son espace dual $W_{\sigma}^{\mathrm{v}}$ puis sur le produit tensoriel $\left(W_{\sigma}^{\mathrm{v}}\right)^{\otimes \ell}$ et, enfin, par quotient, nous obtenons une métrique hermitienne sur $S^{\ell}\left(W_{\sigma}^{\mathrm{v}}\right)$. En outre, les métriques cubistes et celles de Fubini-Study apportent à $\mathcal{M}$ puis à $\varepsilon_{m p}^{*} \mathcal{M}$ une structure de fibré en droites hermitien. Ainsi construit, le fibré vectoriel hermitien $\overline{\mathcal{G}}_{i}$ possède une pente d'Arakelov maximale et la proposition suivante en donne une estimation.

Proposition 5.8. Pour tout entier $i \in\{1, \ldots, N\}$, la pente maximale du fibré vectoriel hermitien $\overline{\mathcal{G}}_{i}$ est inférieure à $\phi_{28} U_{0} / D$, où 
Démonstration. Grâce aux formules (31) et (32) du $\S 1.5$, nous avons la première majoration

$$
\widehat{\mu}_{\max }\left(\overline{\mathcal{G}}_{i}\right) \leq \ell\left(\widehat{\mu}_{\max }\left(\overline{\mathcal{W}^{v}}\right)+2 g \log g\right)+h_{\overline{\mathcal{M}}}\left(\varepsilon_{m p}\right) .
$$

Observons alors que la pente maximale de $\overline{\mathcal{W}}^{\vee}$ admet un majorant qui ne dépend pas de la hauteur de $W_{0}$. En effet, si l'on note $i: t_{\mathcal{A}} \rightarrow \mathcal{W}$ l'application définie par $i(y)=\lambda(y) \oplus y$, les normes $v$-adiques de l'application duale $i^{\vee}: \mathcal{W}^{\vee} \rightarrow t_{\mathcal{A}}^{\vee}$ sont majorées par $(\sqrt{2})^{\epsilon_{v}}$ avec $\epsilon_{v}=1$ si $v$ est archimédienne et $\epsilon_{v}=0$ si $v$ est ultramétrique, car $\|\lambda(y)\|_{v} \leq\|y\|_{v}$, pour tout $y \in t_{\mathcal{A}} \otimes \mathbf{C}_{v}$. Par conséquent, comme $i^{\vee}$ est injective (et même bijective), la première inégalité de pentes du lemme 4.11 fournit la majoration (indépendante de $W_{0}$ )

$$
\widehat{\mu}_{\max }\left(\overline{\mathcal{W}^{v}}\right) \leq \widehat{\mu}_{\max }\left(\overline{t_{\mathcal{A}}^{v}}\right)+\log (2) / 2 .
$$

Par ailleurs, puisque $(A, L)$ est principalement polarisée, nous pouvons appliquer l'inégalité (41) à $\overline{\mathcal{V}}=\bar{t}_{\mathcal{A}}$ (ou bien, aussi, la proposition 2.14 de [33], qui serait plus précise pour la constante numérique), ce qui donne une majoration de la pente maximale de $\overline{t_{\mathcal{A}}^{v}}$. Nous obtenons alors :

$$
\widehat{\mu}_{\max }\left(\overline{\mathcal{W}^{v}}\right) \leq\left(\mathrm{c}_{2}(g)+\log (2) / 2\right) \max \left\{1, h_{F}(A)\right\}
$$

après avoir observé que $h\left(t_{\mathcal{A}}\right)=g \widehat{\mu}\left(\overline{t_{\mathcal{A}}^{v}}\right) \leq h_{F}(A)$ (voir (23), p. 15). De plus, le degré d'Arakelov de $\overline{\varepsilon_{m p}^{*} \mathcal{M}}$ se scinde de la manière suivante

$$
\begin{aligned}
h_{\overline{\mathcal{M}}}\left(\varepsilon_{m p}\right) & =\widehat{\operatorname{deg}}_{\mathrm{n}} \overline{\varepsilon_{m p}^{*} \mathcal{M}} \\
& =D_{0} h_{\overline{\mathcal{O}_{0}(1)}}\left(m u_{0}\right)+3 m^{2}\left(D_{1} \widehat{h}_{L_{1}}\left(p_{1}\right)+\cdots+D_{n} \widehat{h}_{L_{n}}\left(p_{n}\right)\right) .
\end{aligned}
$$

Nous obtenons ainsi

avec

$$
\begin{aligned}
\widehat{\mu}_{\max }\left(\overline{\mathcal{G}}_{i}\right) \leq & c_{27} T \max \left\{1, h_{F}(A)\right\}+3(g+t)^{2} S^{2} \sum_{i=1}^{n} D_{i} \widehat{h}_{L_{i}}\left(p_{i}\right) \\
& +D_{0} \max _{0 \leq m \leq(g+t) S}\left\{h \overline{\mathcal{O}_{\mathcal{X}_{0}}(1)}\left(m u_{0}\right)\right\}
\end{aligned}
$$

$$
\begin{aligned}
c_{27} & :=2(g+t)\left(c_{2}(g)+\log (2) / 2+2 g \log (g)\right) \\
& \leq 30 g^{5}(g+t) \quad\left(\operatorname{car~c} c_{2}(g)=14 g^{5}\right) .
\end{aligned}
$$

Nous en déduisons la majoration

$$
\begin{aligned}
\widehat{\mu}_{\max }\left(\overline{\mathcal{G}}_{i}\right) & \leq c_{27} T \max \left\{1, h_{F}(A)\right\}+3(g+t)^{2} S^{2} \sum_{i=1}^{n} D_{i} \log a_{i}+D_{0} \frac{\log b}{D} \\
& \leq \frac{c_{27} n U_{0}}{c_{12} D}+3(g+t)^{2}\left(\frac{S}{S_{0}}\right)^{2} \frac{n U_{0}}{c_{5} D}+\frac{U_{0}}{c_{44} D}
\end{aligned}
$$

(dans le premier terme de ce majorant apparait un facteur $n$ car $\max \left\{1, h_{F}(A)\right\} \leq n \max \left\{1, \mathrm{~h}_{F}\right\}$ ) et donc

$$
\widehat{\mu}_{\max }\left(\overline{\mathcal{G}}_{i}\right) \leq c_{28} \frac{U_{0}}{D} \quad \text { avec } 2 \text { 28 }:=\frac{c_{27} n}{c_{12}}+\frac{3(g+t)^{2} c_{13}^{2} n}{c_{15}}+\frac{1}{c_{14}} .
$$

La proposition 5.8 en découle après un calcul élémentaire utilisant les estimations

$$
\text { 回 } \geq 15000(g+t) n c \text { 居 et }
$$

5.7. Minoration d'une dimension. À l'instar de la méthode des déterminants d'interpolation de Laurent, la méthode des pentes ne requiert aucune construction de fonction auxiliaire comme cela est courant en transcendance. Néanmoins, il n'y a aucun miracle (!) et la condition de Siegel (nombre d'inconnues comparé au nombre d'équations) est présente quelque part dans la preuve. Le lemme suivant prend en compte précisément ce type de contrainte.

Lemme 5.9. Soit $i_{0}$ l'entier associé au couple $\left(S_{0}+1,0\right)$ de $\nabla$. Alors

$$
\operatorname{dim} E_{i_{0}} \geq \frac{\operatorname{dim} E}{2} \text {. }
$$


Démonstration. Tout d'abord, d'après le choix même de $i_{0}$, il faut noter qu'un élément $s$ quelconque de $E_{i_{0}}$ s'annule le long de $W$ à l'ordre $2(g+t) T$ en tous les points $0, p, \ldots, S_{0} p$. Soit $\Psi$ l'application surjective

$$
\mathrm{H}^{0}\left(\mathbf{P}, \mathcal{O}_{\mathbf{P}}\left(D_{0}, \ldots, D_{n}\right)\right) \otimes_{\sigma_{0}} \mathbf{C} \rightarrow \mathrm{H}^{0}(X, M) \otimes_{\sigma_{0}} \mathbf{C}
$$

considérée au début de la preuve de la proposition 5.3. Son noyau ker $\Psi$ contient l'idéal $I(G)$ des polynômes s'annulant sur $G_{\sigma_{0}}(\mathbf{C})$ et nous avons une suite exacte d'espaces vectoriels complexes

$$
0 \longrightarrow \frac{\operatorname{ker} \Psi}{I(G)} \longrightarrow \frac{\mathrm{H}^{0}\left(\mathbf{P}, \mathcal{O}_{\mathbf{P}}\left(D_{0}, \ldots, D_{n}\right)\right)_{\sigma_{0}}}{I(G)} \stackrel{\widetilde{\Psi}}{\longrightarrow} \mathrm{H}^{0}(X, M)_{\sigma_{0}} \longrightarrow 0 \text {. }
$$

Par définition, la dimension du terme central de cette suite est la fonction de Hilbert-Samuel $H\left(G ; D_{0}, \ldots, D_{n}\right)$ de $G$. L'espace vectoriel $\widetilde{\Psi}^{-1}\left(E_{i_{0}}\right)$ est l'ensemble des polynômes multihomogènes $P$ de degré $\left(D_{0}, \ldots, D_{n}\right)$ tels que $P \circ \iota \circ \exp _{G_{\sigma_{0}}(\mathbf{C})}$ (qui n'est pas identiquement nul dès que $P \neq 0$ mod $I(G))$ s'annule le long de $W$ à l'ordre $2(g+t) T$ en $0, u, \ldots, S_{0} u$. Soit $\rho$ le rang du système linéaire

$$
\forall z \in\left\{0, u, \ldots, S_{0} u\right\}, \mathbf{t} \in \mathbf{N}^{g},|\mathbf{t}| \leq 2(g+t) T, \mathrm{D}_{\mathrm{w}}^{\mathbf{t}}\left(P \circ \iota \circ \exp _{G_{\sigma_{0}}(\mathbf{C})}\right)(z)=0
$$

où $\mathbf{w}=\left(w_{1}, \ldots, w_{g}\right)$ est une base de $W$ et $\frac{1}{\mathbf{t} !} \mathrm{D}_{\mathrm{w}}^{\mathbf{t}}\left(P \circ \iota \circ \exp _{G_{\sigma_{0}}(\mathbf{C})}\right)(z)$ est le $\mathbf{t}^{\mathrm{ème}}$ coefficient de Taylor $\left(P \circ \iota \circ \exp _{G_{\sigma_{0}}(\mathbf{C})}\right)\left(z+z_{1} w_{1}+\cdots+z_{g} w_{g}\right)$. D'une part, par définition de $\rho$, nous avons $\operatorname{dim} \widetilde{\Psi}^{-1}\left(E_{i_{0}, \sigma_{0}}\right)=H\left(G ; D_{0}, \ldots, D_{n}\right)-\rho$, et d'autre part, observons que, grâce à la suite exacte (71), on a

$$
\begin{aligned}
\operatorname{dim} \widetilde{\Psi}^{-1}\left(E_{i_{0}, \sigma_{0}}\right) & \leq \operatorname{dim} E_{i_{0}}+\operatorname{dim}(\operatorname{ker} \Psi) / I(G) \\
& =\operatorname{dim} E_{i_{0}}+H\left(G ; D_{0}, \ldots, D_{n}\right)-\operatorname{dim} E .
\end{aligned}
$$

Ces remarques entraînent $\operatorname{dim} E-\rho \leq \operatorname{dim} E_{i_{0}}$ et il s'agit alors de montrer que $\rho \leq(\operatorname{dim} E) / 2$. La suite de la preuve est classique et repose sur le même argument que celui donné par Philippon et Waldschmidt dans [57]. Comme nous avons besoin d'une estimation effective, les calculs plus précis* du $\S 6.3$ de [20] conviennent mieux à notre situation. Considérons le sous-groupe $\widetilde{G}$ of $G_{\bar{k}}$ défini p. 31, qui réalise le minimum dans la définition de $x$. Alors, en rappelant que $D_{i}^{\prime}=\max \left\{1, D_{i}\right\}$, le rang $\rho$ est majoré par

$$
\begin{aligned}
& \left(\begin{array}{c}
2(g+t) T+\tilde{\lambda} \\
\tilde{\lambda}
\end{array}\right)\left(S_{0}+1\right)\left(\frac{\widetilde{d}(\widetilde{d}+1)}{2}+(\widetilde{d}+1) \mathscr{H}\left(\widetilde{G} ; D_{0}^{\prime}, \ldots, D_{n}^{\prime}\right)\right) \\
& \leq(2(g+t)+1)^{\tilde{\lambda}} \widetilde{T}^{\tilde{\lambda}}\left(S_{0}+1\right) \frac{(\widetilde{d}+2)^{2}}{2} \mathscr{H}\left(\widetilde{G} ; D_{0}^{\prime}, \ldots, D_{n}^{\prime}\right) .
\end{aligned}
$$

Observons alors

$$
\begin{aligned}
\frac{\mathscr{H}\left(\widetilde{G} ; D_{0}^{\prime}, \ldots, D_{n}^{\prime}\right)}{\mathscr{H}\left(G ; D_{0}^{\prime}, \ldots, D_{n}^{\prime}\right)} & =\frac{\left(\begin{array}{c}
\widetilde{d} \\
\tilde{t}
\end{array}\right)}{\left(\begin{array}{c}
g+t \\
t
\end{array}\right)} \times \frac{1}{D_{0}^{t-\widetilde{t}}} \times \frac{\mathscr{H}\left(\widetilde{A} ; 1, \ldots, 1, D_{\kappa}, \ldots, D_{n}\right)}{\mathscr{H}\left(A ; 1, \ldots, 1, D_{\kappa}, \ldots, D_{n}\right)} \\
& \leq \frac{\left(\begin{array}{c}
\widetilde{d} \\
\tilde{t}
\end{array}\right)}{\left(\begin{array}{c}
g+t \\
t
\end{array}\right)} \times \frac{2^{\widetilde{r}}}{\left(x \widetilde{D}_{0}\right)^{t-\widetilde{t}}} \times \frac{\mathscr{H}\left(\widetilde{A} ; \widetilde{D}_{1}, \ldots, \widetilde{D}_{n}\right)}{\mathscr{H}\left(A ; \widetilde{D}_{1}, \ldots, \widetilde{D}_{n}\right)}
\end{aligned}
$$

grâce à la propriété (5) de $\mathscr{H}$ et aux minorations : $1 \geq \widetilde{D}_{i} / 2(1 \leq i \leq \kappa-1), D_{i} \geq \widetilde{D}_{i} / 2(\kappa \leq i \leq n)$ et $D_{0} \geq\left(x \widetilde{D}_{0}\right) / 2$. Ainsi, par définition de $x$ et $\widetilde{G}$, nous obtenons

En outre, notons que

$$
\frac{\mathscr{H}\left(\widetilde{G} ; D_{0}^{\prime}, \ldots, D_{n}^{\prime}\right)}{\mathscr{H}\left(G ; D_{0}^{\prime}, \ldots, D_{n}^{\prime}\right)} \leq \frac{\left(\begin{array}{c}
\widetilde{d} \\
\tilde{t}
\end{array}\right)}{\left(\begin{array}{c}
g+t \\
t
\end{array}\right)} \frac{2^{\widetilde{r}} \sqrt{1 \theta}}{\widetilde{T}^{\widetilde{\lambda}}(S+1)} .
$$

$$
\begin{aligned}
\mathscr{H}\left(G ; D_{0}^{\prime}, \ldots, D_{n}^{\prime}\right) & =\frac{(g+t) !}{t !} 3^{g} D_{0}^{t} D_{\kappa}^{g_{\kappa}} \cdots D_{n}^{g_{n}} \\
& \leq(g+t) ! 3^{g_{1}+\cdots+g_{\kappa-1}} \operatorname{rg} \mathcal{E}
\end{aligned}
$$

${ }^{*}$ Ces calculs sont basés sur le théorème suivant de Chardin 15 : Soit $k$ un corps et $R=k\left[X_{0}, \ldots, X_{n}\right]\left(n \in \mathbf{N}^{*}\right)$. Soit $I$ un idéal homogène de $R$, équidimensionnel et géométriquement réduit. Notons $D \geq 0$ la hauteur de $I$ et $H(I ; \cdot)$ la fonction de Hilbert de $I$. Alors, pour tout $\nu \in \mathbf{N}$, on a $H(I ; \nu) \leq\left(\begin{array}{c}\nu+D \\ D\end{array}\right)+(\operatorname{deg} I-1)\left(\begin{array}{c}\nu+D-1 \\ D\end{array}\right)$. 
(propriété (4) de $\mathscr{H}$ p. 30 et égalité (64) p. 37). Ainsi, nous en déduisons que $\rho$ est inférieur à $c_{29} \operatorname{dim} E$ avec

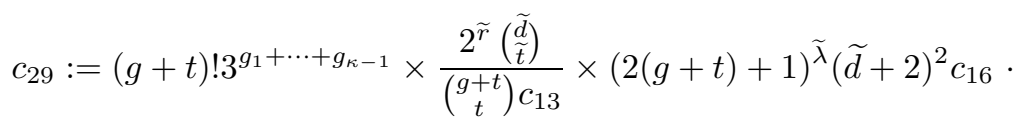

Cette dernière constante est elle-même plus petite que $1 / 2$ comme nous pouvons le constater en

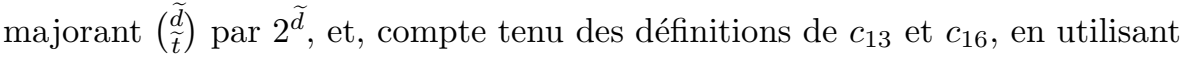

$$
\widetilde{d}+2=\widetilde{t}+\widetilde{g}+2 \leq(t-1)+(g-1)+2=t+g, \quad \tilde{\lambda} \leq g
$$

et $3^{g_{1}+\cdots+g_{\kappa-1}} \leq 3^{g-1}$. Cela termine la preuve.

5.8. Estimations ultramétriques des morphismes d'évaluation. Dans cette partie, nous allons donner une majoration de la norme $\mathfrak{p}$-adique de chacun des morphismes $\varphi_{i}$. Étant donné une section $s \neq 0$ de $M$, nous devons trouver un dénominateur de $\varphi_{i}(s)$ c'est-à-dire, un entier strictement positif $m$ tel que $m \varphi_{i}(s)$ se prolonge en un élément de $\mathcal{G}_{i}$. Lorsque $s$ est une section entière de $\mathcal{M}$, une solution naïve serait $\ell$ ! (un simple calcul local suffit pour voir cela). Malheureusement, cela conduit à un logarithme supplémentaire de la hauteur de $W_{0}$ dans la mesure finale (11) (voir $\S$ 5.1). Comme nous l'avons montré dans le cas général d'un groupe algébrique commutatif (qui n'est pas forcément une variété abélienne) [28], nous devons être plus soigneux pour éviter une telle situation et pour obtenir la dépendance linéaire voulue en la hauteur de $W_{0}$.

Soit $\ell, h$ des entiers strictement positifs. Définissons l'entier

$$
\delta_{\ell}(h):=\operatorname{ppcm}\left\{i_{1} \cdots i_{h^{\prime}} ; 1 \leq h^{\prime} \leq h, i_{j} \in \mathbf{N}^{*}, i_{1}+\cdots+i_{h^{\prime}} \leq \ell\right\} .
$$

Le théorème des nombres premiers implique qu'il existe une constante absolue $c$ telle que

$$
\log \delta_{\ell}(h) \leq \ell \log (c h) .
$$

Bruiltet a montré que $c=4$ convenait (proposition 1 de 14]).

Proposition 5.10. Pour tout entier $i \in\{1, \ldots, N\}$ et toute place finie $\mathfrak{p} \in \operatorname{Spec}\left(\mathcal{O}_{K}\right) \backslash\{(0)\}$, on $a\left\|\delta_{\ell}\left(D_{0}\right) \varphi_{i}\right\|_{\mathfrak{p}} \leq 1$.

Démonstration. Nous reprenons la démonstration du lemme 3.1 donnée dans [27] en l'adaptant à notre contexte. Il y aura une légère simplification car nous n'aurons pas besoin de formules d'addition explicites sur la variété abélienne.

Fixons un entier $i \in\{1, \ldots, N\}$ et un élément $s \neq 0$ de $E_{i} \cap \mathcal{E}$. Nous devons contrôler le dénominateur de $\varphi_{i}(s) \in S^{\ell}\left(W^{\mathrm{v}}\right) \otimes(m p)^{*} M$. En considérant le tiré en arrière de $s$ par la translation $\tau_{\varepsilon_{m p}}: \mathscr{G} \rightarrow \mathscr{G}$, nous pouvons supposer que $m=0$ car

$$
\operatorname{jet}_{W}^{\ell} s\left(\varepsilon_{m p}\right)=\operatorname{jet}_{W}^{\ell}\left(\tau_{\varepsilon_{m p}}^{*} s\right)(\varepsilon) \quad \text { et } \quad \tau_{\varepsilon_{m p}}^{*} s \in \mathcal{E} .
$$

D'autre part, puisque contrôler un dénominateur revient à évaluer chacune de ses normes ultramétriques, nous pouvons localiser en un idéal premier non nul $\mathfrak{p}$ de $\mathcal{O}_{K}$. Notons $\mathcal{O}_{\mathfrak{p}}\left(\subseteq K_{\mathfrak{p}}\right)$ l'anneau de valuation correspondant. Soit alors $\mathscr{U}$ un voisinage ouvert de la section nulle $\varepsilon_{\mathfrak{p}}$ de

$$
\mathcal{A}_{\mathfrak{p}}:=\mathcal{A}_{\operatorname{Spec} \mathcal{O}_{K}}^{\times} \operatorname{Spec} \mathcal{O}_{\mathfrak{p}}
$$

sur lequel le faisceau inversible $\mathcal{L}_{1}^{\otimes 3 D_{1}} \otimes \cdots \otimes \mathcal{L}_{n}^{\otimes 3 D_{n}}$ est trivial, isomorphe à $\mathcal{O}_{\mathcal{A}_{\mathfrak{p}} \mid \mathscr{U}}$. On fixe un tel isomorphisme. De la sorte un élément de $\mathrm{H}^{0}\left(\mathscr{U}, \mathcal{L}_{1}^{\otimes 3 D_{1}} \otimes \cdots \otimes \mathcal{L}_{n}^{\otimes 3 D_{n}}\right)$ s'identifie à un élément de $\mathrm{H}^{0}\left(\mathscr{U}, \mathcal{O}_{\mathcal{A}_{\mathfrak{p}}}\right)$. Soit $\widehat{\mathcal{A}_{\mathfrak{p}}}$ le groupe formel sur $\mathcal{O}_{\mathfrak{p}}$ obtenu par complétion de $\mathcal{A}_{\mathfrak{p}}$ le long de son élément neutre $\varepsilon_{\mathfrak{p}}$. Comme $\mathcal{A}_{\mathfrak{p}} \rightarrow \operatorname{Spec} \mathcal{O}_{\mathfrak{p}}$ est lisse (car $\mathcal{A}$ l'est sur $\mathcal{O}_{K}$ ), il existe des paramètres formels $Y_{1}, \ldots, Y_{g}$ tels que $\mathcal{O}_{\widehat{\mathcal{A}}_{\mathfrak{p}}}$ est isomorphe (au-dessus de $\mathcal{O}_{\mathfrak{p}}$ ) à l'anneau de séries formelles $\mathcal{O}_{\mathfrak{p}}\left[\left[Y_{1}, \ldots, Y_{g}\right]\right]$. Ces paramètres $Y_{1}, \ldots, Y_{g}$ correspondent à un système régulier de paramètres qui engendrent l'idéal de l'immersion fermée $\varepsilon_{\mathfrak{p}}$. Ils s'identifient à des sections locales de $\mathcal{O}_{\mathcal{A}_{\mathfrak{p}}}$ et leurs différentielles $\mathrm{d} Y_{1}, \ldots, \mathrm{d} Y_{g}$ à des sections de $\Omega_{\mathcal{A}_{\mathfrak{p}} / \mathcal{O}_{\mathfrak{p}}}^{1}$. En particulier, il est possible de choisir une base $\left(e_{1}, \ldots, e_{g}\right)$ du $\mathcal{O}_{\mathfrak{p}}$-module libre

$$
t_{\mathcal{A}_{\mathfrak{p}}}=\left(\varepsilon_{\mathfrak{p}}^{*} \Omega_{\mathcal{A}_{\mathfrak{p}} / \mathcal{O}_{\mathfrak{p}}}^{1}\right)^{\vee}
$$


(libre car sans torsion sur un anneau local) telle que les coordonnées $z_{1}, \ldots, z_{g}$ dans cette base soient compatibles avec $Y_{1}, \ldots, Y_{g}$, au sens où $\mathrm{d} z_{i}=\varepsilon_{\mathfrak{p}}^{*} \mathrm{~d} Y_{i}$ pour tout $i \in\{1, \ldots, g\}$. Par ailleurs, le morphisme canonique

$$
\mathrm{H}^{0}\left(\mathcal{A}_{\mathfrak{p}}, \mathcal{O}_{\mathcal{A}_{\mathfrak{p}}}\right) \rightarrow \mathrm{H}^{0}\left(\widehat{\mathcal{A}}_{\mathfrak{p}}, \mathcal{O}_{\widehat{\mathcal{A}}_{\mathfrak{p}}}\right)=\mathcal{O}_{\mathfrak{p}}\left[\left[Y_{1}, \ldots, Y_{g}\right]\right]
$$

permet d'identifier une section de $\mathcal{O}_{\mathcal{A}_{\mathfrak{p}}}$ — et aussi, via la trivialisation fixée, un élément de $\mathrm{H}^{0}\left(\mathscr{U}, \mathcal{L}_{1}^{\otimes 3 D_{1}} \otimes \cdots \otimes \mathcal{L}_{n}^{\otimes 3 D_{n}}\right)$ — à une série formelle en les $Y_{i}$ à coefficients dans $\mathcal{O}_{\mathfrak{p}}$.

De la même manière, soit $X_{1}, \ldots, X_{t}$ une base du $\mathcal{O}_{\mathfrak{p}}$-module libre $\left(t_{\mathcal{A}} / \mathcal{W}_{0}\right)^{\mathrm{v}} \otimes_{\mathcal{O}_{K}} \mathcal{O}_{\mathfrak{p}}$ et $X_{0}=1$

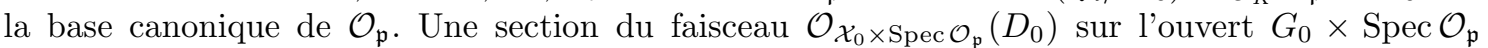
s'identifie à un polynôme en $X_{1}, \ldots, X_{t}$ de degré inférieur à $D_{0}$ et à coefficients dans $\mathcal{O}_{\mathfrak{p}}$, après trivialisation de $\mathcal{O}_{\mathcal{X}_{0} \times \operatorname{Spec} \mathcal{O}_{\mathfrak{p}}}\left(D_{0}\right)$ sur l'ouvert en question au moyen de $X_{0}^{D_{0}}$.

Grâce à tous ces choix, la section $s \otimes 1$ de $\mathcal{M} \otimes \mathcal{O}_{\mathfrak{p}}$ peut se voir (localement sur le produit des ouverts $G_{0} \times \operatorname{Spec} \mathcal{O}_{\mathfrak{p}}$ et $\left.\mathscr{U}\right)$ comme une série formelle

$$
s \otimes 1=\sum \theta_{\mathbf{i}, \mathbf{j}} \mathbf{X}^{\mathbf{i}} \mathbf{Y}^{\mathbf{j}} \quad \text { avec } \theta_{\mathbf{i}, \mathbf{j}} \in \mathcal{O}_{\mathfrak{p}} \text { et } \begin{gathered}
\mathbf{X}=\left(X_{1}, \ldots, X_{t}\right) \\
\mathbf{Y}=\left(Y_{1}, \ldots, Y_{g}\right)
\end{gathered} .
$$

où $\mathbf{i} \in \mathbf{N}^{t}, \mathbf{j} \in \mathbf{N}^{g}$ et de plus, $\theta_{\mathbf{i}, \mathbf{j}}=0$ pour $|\mathbf{i}| \geq D_{0}+1$. Soit

$$
\widehat{\log }: \widehat{A}_{K_{\mathfrak{p}}} \rightarrow t_{A} \otimes_{K_{\mathfrak{p}}} \mathcal{O}_{\widehat{A}_{K_{\mathfrak{p}}}}
$$

le logarithme formel du groupe formel $\widehat{A}_{K_{\mathfrak{p}}}$ sur $K_{\mathfrak{p}}$. Il définit un isomorphisme de groupes formels (voir [38]). Sa différentielle est invariante par l'action de la loi de groupe formelle de $\widehat{\mathcal{A}}_{\mathfrak{p}}$ et elle est définie sur $\mathcal{O}_{\mathfrak{p}}$

$$
\mathrm{d}(\widehat{\log }) \in \mathrm{H}^{0}\left(\widehat{\mathcal{A}}_{\mathfrak{p}}, \Omega_{\widehat{\mathcal{A}}_{\mathfrak{p}} / \mathcal{O}_{\mathfrak{p}}} \otimes t_{\mathcal{A}}\right)
$$

En termes des coordonnées introduites, nous pouvons décrire l'application $\widehat{\log }$ à l'aide d'un $g$-uplet $\left(\ell_{1}, \ldots, \ell_{g}\right)$ de séries formelles dont les différentielles vérifient

$$
\mathrm{d} \ell_{i} \in \sum_{h=1}^{g} \mathcal{O}_{\mathfrak{p}}\left[\left[Y_{1}, \ldots, Y_{g}\right]\right] \mathrm{d} Y_{h}
$$

et $\ell_{i}(\mathbf{Y})=Y_{i} \bmod \left(Y_{1}, \ldots, Y_{g}\right)^{2}$ car $\mathrm{d} z_{i}=\varepsilon_{\mathfrak{p}}^{*} \mathrm{~d} Y_{i}$. Ainsi, pour tout $\mathbf{n}=\left(n_{1}, \ldots, n_{g}\right) \in \mathbf{N}^{g}$ et tout entier $i \in\{1, \ldots, g\}$, le coefficient $a_{\mathbf{n}}^{(i)}$ de $\mathbf{Y}^{\mathbf{n}}=Y_{1}^{n_{1}} \cdots Y_{g}^{n_{g}}$ dans $\ell_{i}$ a la propriété suivante :

$$
\forall j \in\{1, \ldots, g\}, \quad n_{j} a_{\mathbf{n}}^{(i)} \in \mathcal{O}_{\mathfrak{p}} .
$$

En notant $\left(\lambda_{l, j}\right) \in \operatorname{Mat}_{t, g}\left(\mathcal{O}_{\mathfrak{p}}\right)$ la matrice de l'application linéaire $\lambda_{\mathfrak{p}}: t_{\mathcal{A}_{\mathfrak{p}}} \rightarrow\left(t_{\mathcal{A}} / \mathcal{W}_{0}\right) \otimes \mathcal{O}_{\mathfrak{p}}$ dans les coordonnées $\left(z_{1}, \ldots, z_{g}\right)$ et $\left(X_{1}, \ldots, X_{t}\right)$, les équations qui définissent $\mathcal{W}$ sont

$$
X_{l}=\sum_{h=1}^{g} \lambda_{l, h} z_{h}, \quad l \in\{1, \ldots, t\}
$$

(nous avons identifié $\left(z_{1}, \ldots, z_{g}\right)$ à la base duale de $\left.\left(e_{1}, \ldots, e_{g}\right)\right)$. Celles qui définissent le sous-groupe de Lie formel $\widehat{\exp }_{\mathscr{G}_{\mathrm{p}}} \mathcal{W}$ de $\widehat{\mathcal{X}}_{\mathfrak{p}}$ sont donc

$$
X_{l}=\sum_{h=1}^{g} \lambda_{l, h} \ell_{h}(\mathbf{Y})
$$

Le passage des variables $\left(z_{1}, \ldots, z_{g}\right)$ aux variables $\left(Y_{1}, \ldots, Y_{g}\right)$ au moyen $d u$ logarithme formel ne change pas la valuation d'une série formelle en l'un ou l'autre uplet de ces variables, ni même le premier polynôme de Taylor non nul de la série en question, car $z_{i}=Y_{i}+$ termes d'ordre $\geq 2$. Si nous ajoutons à cette observation le fait que $s$ s'annule à l'ordre $\ell$ le long de $\mathcal{W}$ en l'origine, alors

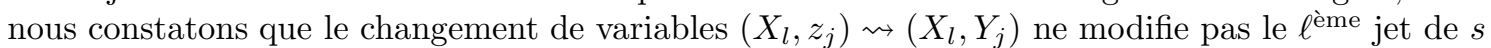
le long de $\mathcal{W}$ en 0 . De la sorte, $\varphi_{i}(s)$ s'identifie à la partie homogène de degré $\ell$ de la série

$$
\sum_{|\mathbf{i}| \leq D_{0}} \theta_{\mathbf{i}, \mathbf{j}} \mathbf{Y}^{\mathbf{j}} \prod_{l=1}^{t}\left(\sum_{h=1}^{g} \lambda_{l, h} \ell_{h}(\mathbf{Y})\right)^{i_{l}}
$$


(dans cette somme, $\mathbf{i}=\left(i_{1}, \ldots, i_{t}\right) \in \mathbf{N}^{t}$ et $\left.\mathbf{j} \in \mathbf{N}^{g}\right)$. Posons

$$
b_{\mathbf{n}}^{(l)}:=\sum_{h=1}^{g} \lambda_{l, h} a_{\mathbf{n}}^{(h)}
$$

le coefficient devant $\mathbf{Y}^{\mathbf{n}}$ de la série $\sum_{h=1}^{g} \lambda_{l, h} \ell_{h}(\mathbf{Y})$. Les coefficients du polynôme homogène de degré $\ell$ de la série (75) sont des sommes finies de termes de la forme

$$
\theta_{\mathbf{i}, \mathbf{j}} \prod_{h=1}^{|\mathbf{i}|} b_{\mathbf{n}_{h}}^{\left(m_{h}\right)}
$$

où $m_{h} \in\{1, \ldots, t\}, \mathbf{n}_{h} \in \mathbf{N}^{g}$ et $\sum_{h}\left|\mathbf{n}_{h}\right|=\ell$. Soit $n_{h}$ une composante non nulle (quelconque) de $\mathbf{n}_{h}$. D'après (74), l'entier $n_{h}$ est un dénominateur de $b_{\mathbf{n}_{h}}^{\left(m_{h}\right)}$. Nous en déduisons que le produit $n_{1} \cdots n_{\mid \mathbf{i}}$ est un dénominateur de $(76)$. Comme c'est un produit d'au plus $|\mathbf{i}| \leq D_{0}$ entiers strictement positifs dont la somme est au plus $\ell$ (car $\left.n_{h} \leq\left|\mathbf{n}_{h}\right|\right)$, il divise l'entier $\delta_{\ell}\left(D_{0}\right)$, par définition même de cet entier construit comme un ppcm. Ainsi l'élément $\delta_{\ell}\left(D_{0}\right) \varphi_{i}(s)$ appartient à $\mathcal{O}_{\mathfrak{p}}\left[Y_{1}, \ldots, Y_{g}\right]_{\ell}$ et, donc, également à $\mathcal{O}_{\mathfrak{p}}\left[z_{1}, \ldots, z_{g}\right]_{\ell}=S^{\ell}\left(\mathcal{W}^{\vee}\right) \otimes_{\mathcal{O}_{K}} \mathcal{O}_{\mathfrak{p}}$.

5.9. Majorations des normes archimédiennes des morphismes d'évaluation. Dans ce paragraphe, étant donné un plongement $\sigma: K \hookrightarrow \mathbf{C}$ et un entier $i \in\{1, \ldots, N\}$, nous donnons une majoration de la norme d'opérateur $\left\|\varphi_{i}\right\|_{\sigma}$ du morphisme $\varphi_{i}$. Comme nous l'avons déjà dit au $\S$ 5.1, il s'avère particulièrement important que la factorielle de l'ordre de dérivation n'apparaisse pas au cours de la preuve. C'est pourquoi nous allons commencer par décrire de manière précise les morphismes $\varphi_{i}$ et les normes d'opérateur $\|\cdot\|_{\sigma}$. Signalons que les détails qui vont suivre pour les estimations de $\left\|\varphi_{i}\right\|_{\sigma}$ sont proches de ceux de la preuve de la proposition 2.13 de 33 .

Soit $\exp _{\sigma}: t_{G_{\sigma}}(\mathbf{C}) \rightarrow \mathcal{X}_{\sigma}(\mathbf{C})$ l'application exponentielle du groupe de Lie complexe $G_{\sigma}(\mathbf{C})$, prolongée à $\mathcal{X}_{\sigma}(\mathbf{C})$. Alors le fibré vectoriel $\exp _{\sigma}^{*} M_{\sigma}$ sur $t_{G_{\sigma}}(\mathbf{C})$ est trivial. Choisissons un isomorphisme $\nu: \exp _{\sigma}^{*} M_{\sigma} \rightarrow \mathcal{O}_{t_{G_{\sigma}}}(\mathbf{C})$ tel que, pour tout $s \in E_{\sigma}:=E \otimes_{\sigma} \mathbf{C}$, la fonction holomorphe $\nu\left(\exp _{\sigma}^{*} s\right)$ sur $t_{G_{\sigma}}(\mathbf{C})$ satisfasse

$$
\forall z=z_{0} \oplus \cdots \oplus z_{n} \in t_{G_{\sigma}}(\mathbf{C})=\left(t_{A} / W_{0}\right)_{\sigma}(\mathbf{C}) \stackrel{\perp}{\oplus} t_{A_{1, \sigma}}(\mathbf{C}) \stackrel{\perp}{\oplus} \cdots \stackrel{\perp}{\oplus} t_{A_{n, \sigma}}(\mathbf{C})
$$

et $x=\exp _{\sigma}(z)$,

$$
\|s(x)\|_{\overline{x^{*} \mathcal{M}}, \sigma}=\frac{\left|\nu\left(\exp _{\sigma}^{*} s\right)(z)\right|}{\left(1+\left\|z_{0}\right\|_{\sigma}^{2}\right)^{D_{0} / 2}} \times \exp \left\{-\frac{3 \pi}{2}\left(\sum_{j=1}^{n} D_{j}\left\|z_{j}\right\|_{L_{j}}^{2}, \sigma\right)\right.
$$

(voir la discussion du $\S 4.2$. Cette égalité nous permet de travailler avec «d'authentiques » fonctions holomorphes et toutes les estimations impliquant les $\sigma$-normes de $s(x)$ (ou de $s$ ) seront effectuées avec $\nu\left(\exp _{\sigma}^{*} s\right)$.

Fixons un entier $i \in\{1, \ldots, N\}$ et soit $(m, \ell) \in \nabla$ le couple associé à $i$ (voir p. 39). Considérons un élément non nul $s \in E_{i} \otimes_{\sigma} \mathbf{C}$. L'image de $s$ par $\varphi_{i}$ appartient à $S^{\ell}\left(W^{\vee}\right) \otimes(m p)^{*} M \otimes_{\sigma} \mathbf{C}$, espace isomorphe à $\operatorname{Hom}_{\mathbf{C}}\left(S^{\ell} W_{\sigma},(m p)^{*} M_{\sigma}\right)$. Cependant cet isomorphisme n'est pas isométrique comme nous l'avons vu au lemme 4.1. Ainsi, avec la notation $\Theta_{\ell}$ de ce lemme, nous pouvons considérer l'application $\Theta_{\ell}\left(\varphi_{i}(s)\right)_{\sigma}: S^{\ell} W_{\sigma} \rightarrow(m p)^{*} M_{\sigma}, \mathrm{D} \mapsto(\mathrm{D} s)(m p)$ où $\mathrm{D}$ est vue comme une dérivation (d'ordre $\ell$ ) le long de $W_{\sigma}$. On a donc

$$
\begin{aligned}
& \left\|\varphi_{i}(s)\right\|_{\sigma} \leq\left\|\Theta_{\ell}\left(\varphi_{i}(s)\right)\right\|_{\sigma}
\end{aligned}
$$

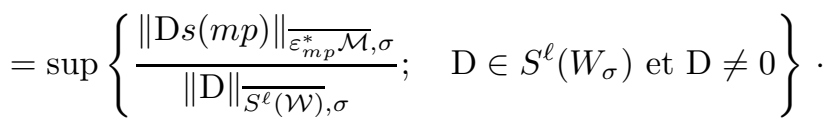

Soit $\left(w_{1}, \ldots, w_{g}\right)$ une base orthonormée de $W_{\sigma}$. En écrivant

$$
\mathrm{D}=\sum_{\substack{\mathbf{i} \in \mathbf{N} g \\|\mathbf{i}|=\ell}} d_{\mathbf{i}} w_{1}^{i_{1}} \otimes \cdots \otimes w_{g}^{i_{g}}
$$

on a

$$
\|\mathrm{D}\| \frac{2}{S^{\ell}(\mathcal{W}), \sigma}=\sum_{|\mathbf{i}|=\ell}\left|d_{\mathbf{i}}\right|^{2} \frac{\mathbf{i} !}{\ell !} \geq\left(\sum_{|\mathbf{i}|=\ell}\left|d_{\mathbf{i}}\right|\right)^{2} \times g^{-\ell} .
$$


Le nombre $\|\mathrm{D} s(m p)\|_{\overline{\varepsilon_{m p}^{*} \mathcal{M}}, \sigma}$ est alors plus petit que

$$
\sum_{|\mathbf{i}|=\ell}\left|d_{\mathbf{i}}\right| \times\left\|w_{1}^{i_{1}} \otimes \cdots w_{g}^{i_{g}} s(m p)\right\|_{\varepsilon_{m p}^{*} \mathcal{M}}, \sigma
$$

ce qui entraîne

$$
\left\|\varphi_{i}(s)\right\|_{\sigma} \leq g^{\ell / 2} \max _{|\mathbf{i}|=\ell}\left\{\left\|w_{1}^{i_{1}} \otimes \cdots \otimes w_{g}^{i_{g}} s(m p)\right\|_{\varepsilon_{m p}^{*} \mathcal{M}, \sigma}\right\}
$$

Soit $u^{\prime}:=m u_{0} \oplus u_{1}^{\prime} \oplus \cdots \oplus u_{n}^{\prime}\left(\operatorname{avec} u_{j}^{\prime} \in t_{A_{j, \sigma}}(\mathbf{C})\right)$ un logarithme de $m \sigma(p)$ tel que

$$
r_{\sigma}\left(m p_{1}, \ldots, m p_{n}\right)=\left\|u_{1}^{\prime} \oplus \cdots \oplus u_{n}^{\prime}\right\|_{\sigma}
$$

(la définition de $r_{\sigma}$ est donnée au $\S$ 4.7). Avec ce logarithme et la formule (78) l'on peut calculer la norme de $w_{1}^{i_{1}} \otimes \cdots \otimes w_{g}^{i_{g}} s(m p)$ et, via (79), on a

$$
\begin{aligned}
\left\|\varphi_{i}(s)\right\|_{\sigma} \leq & \frac{g^{\ell / 2}}{\left(1+\left\|m u_{0}\right\|_{\sigma}^{2}\right)^{D_{0} / 2}} \exp \left\{-\frac{3 \pi}{2} \sum_{j=1}^{n} D_{j}\left\|u_{j}^{\prime}\right\|_{\sigma}^{2}\right\} \\
& \times \max _{|\mathbf{i}|=\ell}\left\{\left|\frac{1}{\mathbf{i} !}\left(\frac{\partial}{\partial \mathbf{z}}\right)^{\mathbf{i}} \nu\left(\exp _{\sigma}^{*} s\right)\left(u^{\prime}+z_{1} w_{1}+\cdots+z_{g} w_{g}\right)_{\mid \mathbf{z}=0}\right|\right\} .
\end{aligned}
$$

Nous pouvons majorer le dernier terme de 80 avec l'inégalité de Cauchy appliquée à la fonction holomorphe $\nu\left(\exp _{\sigma}^{*} s\right)$. Pour tout nombre réel strictement positif $r$, on a

$$
\begin{aligned}
& \max _{|\mathbf{i}|=\ell}\left\{\left|\frac{1}{\mathbf{i} !}\left(\frac{\partial}{\partial \mathbf{z}}\right)^{\mathbf{i}} \nu\left(\exp _{\sigma}^{*} s\right)\left(u^{\prime}+z_{1} w_{1}+\cdots+z_{g} w_{g}\right)_{\mathbf{z}=0}\right|\right\} \\
& \leq \frac{1}{r^{\ell}} \sup _{\substack{x \in W_{\sigma} \\
\|x\|_{\sigma \leq r}}}\left|\nu\left(\exp _{\sigma}^{*} s\right)\left(u^{\prime}+x\right)\right| \\
& \leq \frac{1}{r^{\ell}} \sup _{\substack{x \in t_{G \sigma}(\mathbf{C}) \\
\|x\|_{\sigma \leq r}}}\left|\nu\left(\exp _{\sigma}^{*} s\right)\left(u^{\prime}+x\right)\right|
\end{aligned}
$$

et avec la formule (78), nous pouvons récrire ce majorant au moyen de $\|s\|_{\infty, \sigma}$, ce qui donne pour $\varphi_{i}(s)$ la borne

$$
\begin{aligned}
\left\|\varphi_{i}(s)\right\|_{\sigma} \leq & \frac{g^{\ell / 2}}{r^{\ell}}\left(\frac{1+\left(\left\|m u_{0}\right\|_{\sigma}+r\right)^{2}}{1+\left\|m u_{0}\right\|_{\sigma}^{2}}\right)^{D_{0} / 2} \\
& \times \exp \left\{\frac{3 \pi r}{2} \sum_{j=1}^{n} D_{j}\left(2\left\|u_{j}^{\prime}\right\|_{\sigma}+r\right)\right\}\|s\|_{\infty, \sigma} .
\end{aligned}
$$

Choisissons

$$
r:=\frac{\sqrt{g}}{\max \left\{1, r_{\sigma}\left(m p_{1}, \ldots, m p_{n}\right)\right\}} .
$$

À l'aide des estimations

$$
\frac{1+\left(\left\|m u_{0}\right\|_{\sigma}+r\right)^{2}}{1+\left\|m u_{0}\right\|_{\sigma}^{2}} \leq 1+r+r^{2} \leq 3 g
$$

et

$$
\sum_{j=1}^{n} D_{j}\left(2\left\|u_{j}^{\prime}\right\|_{\sigma}+r\right) \leq n D_{n}\left(2 r_{\sigma}\left(m p_{1}, \ldots, m p_{n}\right)+r\right),
$$

car $D_{i} \leq D_{n}$ pour $i \in\{1, \ldots, n\}$, nous déduisons de (82) la majoration

$$
\left\|\varphi_{i}\right\|_{\sigma} \leq(3 g)^{D_{0} / 2} \max \left\{1, r_{\sigma}\left(m p_{1}, \ldots, m p_{n}\right)\right\}^{\ell} e^{\frac{9 \pi}{2} n g D_{n}} \max _{\substack{s \in \mathcal{E}_{\sigma} \\ s \neq 0}}\left\{\frac{\|s\|_{\infty, \sigma}}{\|s\|_{\overline{\mathcal{E}}, \sigma}}\right\} \text {. }
$$

Compte tenu du choix des paramètres et, en particulier, des majorations $D_{0} \leq U_{0} /\left(\emptyset_{14} D\right)$ et $D_{n} \leq U_{0} /(c \sqrt{12} 215 D)$, nous obtenons alors l'estimation générale suivante. 
Proposition 5.11. Pour tout plongement complexe $\sigma: K \hookrightarrow \mathbf{C}$ et tout entier $i \in\{1, \ldots, N\}$, on $a$

avec

$$
\left\|\varphi_{i}\right\|_{\sigma} \leq \max \left\{1, r_{\sigma}\left(m p_{1}, \ldots, m p_{n}\right)\right\}^{\ell} e^{c_{30} U_{0} / D} \times \max _{\substack{s \in \mathcal{E}_{\sigma} \\ s \neq 0}}\left\{\frac{\|s\|_{\infty, \sigma}}{\|s\|_{\overline{\mathcal{E}}, \sigma}}\right\}
$$

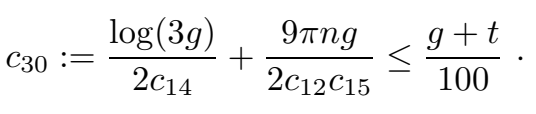

5.10. Majorations fines des normes archimédiennes des morphismes d'évaluation. Dans les estimations du paragraphe précédent, nous n'avons pas tenu compte de toute l'information contenue dans les éléments de $E_{i}$. Bien sûr, l'appartenance de $s$ à $E_{i}$ a permis de définir $\varphi_{i}(s)$, mais pour cela l'annulation des premiers jets jusqu'à l'ordre $\ell$ de $s$ le long de $W$ au (seul) point $m p$ aurait été suffisante. Ici, nous allons employer un lemme d'interpolation qui prend mieux en compte les propriétés d'annulation de $s$. D'une certaine manière, tout le sel de la méthode de Baker réside dans les estimations de ce paragraphe.

Dans toute la suite, nous ne considérons que des plongements archimédiens $\sigma: K \hookrightarrow \mathbf{C}$ au-dessus de $\sigma_{0}: k \hookrightarrow \mathbf{C}$. Par ailleurs, nous reprenons certaines des notations de la section précédente. Fixons un entier $i \in\left\{i_{0}, \ldots, N\right\},(m, \ell) \in \nabla$ le couple associé à $i$, un élément non nul $s$ de $E_{i, \sigma}$ et notons $\vartheta$ la fonction entière $\nu\left(\exp _{\sigma}^{*} s\right)$ définie sur $t_{G_{\sigma}}(\mathbf{C})$. Soit $w:=\left(w_{1}, \ldots, w_{g}\right)$ une base orthonormée de $W_{\sigma}$. Au moyen de l'hypothèse

$$
\vartheta\left(m^{\prime} u+z_{1} w_{1}+\cdots+z_{g} w_{g}\right) \in\left(z_{1}, \ldots, z_{g}\right)^{2(g+t) T+1} \quad \text { pour } \quad m^{\prime} \in\left\{0, \ldots, S_{0}\right\},
$$

il s'agit de fournir une majoration précise des dérivées de $\vartheta$ le long de $W$ à l'ordre $\ell$ en $m p$, qui fasse intervenir la distance de $u$ à $W_{\mathbf{C}}$. Pour cela, définissons

$$
w:=\lambda\left(u_{1} \oplus \cdots \oplus u_{n}\right) \oplus u_{1} \oplus \cdots \oplus u_{n} \in W_{\sigma} .
$$

Si $(\lambda(\mathbf{u}), \mathbf{u})$ est le vecteur de $W_{\mathbf{C}}$ qui réalise la distance de $u$ à $W_{\mathbf{C}}$ :

$$
\mathrm{d}\left(u, W_{\mathbf{C}}\right)^{2}=\left\|u_{0}-\lambda(\mathbf{u})\right\|^{2}+\|u-\mathbf{u}\|^{2}
$$

alors l'inégalité triangulaire

$$
\left\|u_{0}-\lambda(u)\right\| \leq\left\|u_{0}-\lambda(\mathbf{u})\right\|+\|\lambda(\mathbf{u}-u)\|
$$

entraîne $\|u-w\|_{\sigma} \leq \sqrt{2} \mathrm{~d}\left(u, W_{\mathbf{C}}\right)$. De plus, par définition $\operatorname{des} \log a_{i} \leq \log a_{1}$, on a

$$
\|w\|_{\sigma}^{2} \leq 2 \sum_{i=1}^{n}\left\|u_{i}\right\|_{\sigma_{0}}^{2} \leq 2 n \frac{D \log a_{1}}{\mathfrak{e}^{2}} \leq g \frac{D \log a_{1}}{\log \mathfrak{e}} \leq g e^{2 \mathfrak{a} \log \mathfrak{e}}
$$

et donc

$$
\begin{aligned}
2(g+t) T \log \left(g+\sqrt{g}\|w\|_{\sigma}\right) & \leq 2(g+t) T \log \left(g+g e^{\mathfrak{a} \log \mathfrak{e}}\right) \\
& \leq 2(g+t)(1+\log (2 g)) T \mathfrak{a} \log \mathfrak{e} \\
& \leq c_{31} U_{0} \quad \text { avec } c_{31}:=\frac{2(g+t)(1+\log (2 g))}{c_{12}} .
\end{aligned}
$$

Cette estimation ainsi que la borne (large) $c_{31} \leq g^{2}(g+t) / 10$ seront utiles un peu plus tard (estimation (89)).

Afin de simplifier la présentation, il est commode de raisonner par l'absurde pour démontrer le théorème 5.2. C'est pourquoi nous supposerons à partir de maintenant et jusqu'à la fin de la preuve que l'inégalité suivante est satisfaite :

$$
\text { (H) } \quad \log \mathrm{d}\left(u, W_{\mathbf{C}}\right)<-\varnothing U_{0} .
$$

Observons que cette hypothèse implique $8(g+t) S \mathfrak{e} \mathrm{d}\left(u, W_{\mathbf{C}}\right) \leq 1$ si nous supposons

car on a

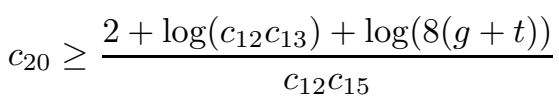

$$
\begin{aligned}
& \log (8(g+t) S \mathfrak{e}) \leq \log (8(g+t) \not 12(13)+\log \mathfrak{a}+\log \mathfrak{e} \\
& \leq(\log (8(g+t) d \sqrt{12} \sqrt{13})+2 \mathfrak{a} \log \mathfrak{e}) D_{n} \\
& \leq\left(\frac{2+\log \left(c_{12}\left(c_{13}\right)+\log (8(g+t))\right.}{d_{12} c_{15}}\right) U_{0} \text {. }
\end{aligned}
$$


La condition (84) sera automatiquement satisfaite à la fin de la preuve. Mais elle est d'ores et déjà vraie si nous prenons $\phi_{20}=24 g^{2}(g+t)$. Soulignons que l'hypothèse $(\mathbf{H})$ n'est pas à proprement parler nécessaire pour continuer la démonstration mais elle est un moyen commode pour la présenter.

Tout d'abord, nous avons besoin du lemme de comparaison suivant.

Lemme 5.12. Pour tout $\mathbf{t} \in \mathbf{N}^{g}$ de longueur $\leq 2(g+t) T$ et tout entier $m^{\prime} \in\{0, \ldots,(g+t) S\}$, on $a$

$$
\left|\frac{1}{\mathbf{t} !} \mathrm{D}_{\mathrm{w}}^{\mathbf{t}} \vartheta\left(m^{\prime} u\right)-\frac{1}{\mathbf{t} !} \mathrm{D}_{\mathrm{w}}^{\mathbf{t}} \vartheta\left(m^{\prime} w\right)\right| \leq e^{-c_{32} U_{0}}\left(1+\left(m^{\prime}\left\|u_{0}\right\|_{\sigma}\right)^{2}\right)^{D_{0} / 2}\|s\|_{\infty, \sigma}
$$

où c $c_{32}$ est une constante $\geq(23,1) g^{2}(g+t)$.

Démonstration. La preuve de cet énoncé repose sur l'inégalité des accroissements finis appliquée à la fonction d'une variable réelle $h:[0,1] \rightarrow \mathbf{C}$ définie par

$$
h(x):=\frac{1}{\mathbf{t} !}\left(\mathrm{D}_{\mathrm{w}}^{\mathbf{t}} \vartheta\right)\left(m^{\prime} u+x m^{\prime}(w-u)\right) .
$$

En effet, soit e $:=\left(e_{1}, \ldots, e_{g+t}\right)$ une base orthonormée de $t_{G_{\sigma}}(\mathbf{C})$, compatible avec la décomposition hermitienne (77) (c'est-à-dire que $\left(e_{1}, \ldots, e_{t}\right)$ est une base de $\left(t_{A} / W_{0}\right)_{\sigma}$ et, si l'on pose $g_{0}:=0$, $\left(e_{t+g_{1}+\cdots+g_{i-1}+1}, \ldots, e_{t+g_{1}+\cdots+g_{i}}\right)$ est une base de $t_{A_{i, \sigma}}(\mathbf{C})$, pour $\left.1 \leq i \leq n\right)$. Soit $\theta_{1}, \ldots, \theta_{g+t}$ les composantes de $w-u$ dans e (en particulier, $\sum_{i=1}^{g+t}\left|\theta_{i}\right|^{2}=\|w-u\|_{\sigma}^{2}$ ). La dérivée première de $h$ est

$$
h^{\prime}(x)=\sum_{i=1}^{g+t} m^{\prime} \theta_{i} \frac{1}{\mathbf{t} !}\left(\mathrm{D}_{e_{i}} \mathrm{D}_{\mathrm{w}}^{\mathbf{t}} \vartheta\right)\left(m^{\prime} u+x m^{\prime}(w-u)\right) .
$$

Grâce à l'inégalité des accroissements finis

$$
|h(1)-h(0)| \leq \max _{x \in[0,1]}\left|h^{\prime}(x)\right|,
$$

il suffit de borner uniformément $h^{\prime}$ sur l'intervalle [0,1]. Écrivons chacun des vecteurs $w_{i}(1 \leq i \leq$ $g$ ) comme une combinaison linéaire des $e_{j}$ et développons les dérivées $\mathrm{D}_{e_{i}} \mathrm{D}_{\mathrm{w}}^{\mathbf{t}}$ en termes des $\mathrm{D}_{\mathrm{e}}^{\overline{\mathbf{j}}}$, $\mathbf{j} \in \mathbf{N}^{g+t},|\mathbf{j}|=|\mathbf{t}|+1$. Ainsi, au moyen de l'inégalité de Cauchy employée comme dans (81) (avec $r=1$ ), nous avons :

$$
\begin{aligned}
\left|h^{\prime}(x)\right| & \leq m^{\prime}\left(\sum_{i=1}^{g+t}\left|\theta_{i}\right|\right) \times \max _{1 \leq i \leq g+t}\left|\frac{\mathrm{D}_{e_{i}} \mathrm{D}_{\mathrm{w}}^{\mathbf{t}}}{\mathbf{t} !} \vartheta\left(m^{\prime} u+x m^{\prime}(w-u)\right)\right| \\
& \leq(g+t)^{\frac{3}{2}(|\mathbf{t}|+1)} S\|w-u\|_{\sigma} \max _{|\mathbf{i}|=|\mathbf{t}|+1}\left|\frac{\mathrm{D}_{\mathbf{e}}^{\mathbf{i}}}{\mathbf{i} !} \vartheta\left(m^{\prime} u+x m^{\prime}(w-u)\right)\right| \\
& \leq(g+t)^{\frac{9}{2}(g+t) T} S\|w-u\|_{\substack{\sigma \in_{t}(\mathbf{C}),\|z\|_{\sigma \leq 1}}}\left|\vartheta\left(m^{\prime} u+x m^{\prime}(w-u)+z\right)\right| .
\end{aligned}
$$

La projection de $m^{\prime} u+x m^{\prime}(w-u)+z \operatorname{sur}\left(t_{A} / W_{0}\right)_{\sigma}$ est un vecteur de norme inférieure à

$$
m^{\prime}\left\|u_{0}\right\|_{\sigma}+m^{\prime}\|w-u\|_{\sigma}+1 \leq m^{\prime}\left\|u_{0}\right\|_{\sigma}+2
$$

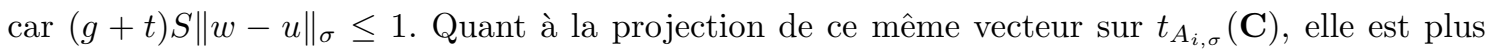
simplement majorer en norme par $m^{\prime}\left\|u_{i}\right\|_{\sigma}+1$ car $w_{i}=u_{i}$. Avec la formule (78), nous pouvons écrire le dernier maximum de $\sqrt{86}$ ) en fonction de la norme infinie de $s$ et nous obtenons alors :

$$
\begin{aligned}
\left|h^{\prime}(x)\right| \leq & (g+t)^{\frac{9}{2}(g+t) T} S\|w-u\|_{\sigma} \times\left(1+\left(m^{\prime}\left\|u_{0}\right\|_{\sigma}+2\right)^{2}\right)^{D_{0} / 2} \\
& \times \exp \left\{\frac{3 \pi}{2} \sum_{i=1}^{n} D_{i}\left(m^{\prime}\left\|u_{i}\right\|_{\sigma_{0}}+1\right)^{2}\right\} \times\|s\|_{\infty, \sigma} .
\end{aligned}
$$

Grâce à $(x+y)^{2} \leq 2\left(x^{2}+y^{2}\right)$, la quantité $\frac{3 \pi}{2} \sum_{i=1}^{n} D_{i}\left(m^{\prime}\left\|u_{i}\right\|_{\sigma_{0}}+1\right)^{2}$ est majorée par

$$
3 \pi\left(\sum_{i=1}^{n} D_{i}\left((g+t) S\left\|u_{i}\right\|_{\sigma_{0}}\right)^{2}\right)+n D_{n}
$$


Nous concluons avec le lemme 5.1, (v) et (vi) et la constante $c_{32}$ de (85) est

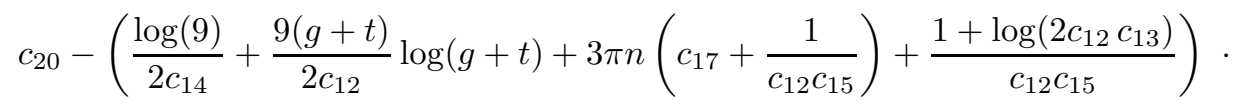

La minoration de $c_{32}$ découle des estimations $14 \geq 7200$ et $\ell_{15} \geq 9600 \pi$.

Soit $\mathbf{t} \in \mathbf{N}^{g}$ un $g$-uplet d'entiers de longueur $\ell$. Comme $i \geq i_{0}$, on a $|\mathbf{t}| \leq(g+t) T$. Soit f la fonction analytique d'une variable complexe définie par

$$
\mathrm{f}(z):=\frac{1}{\mathbf{t} !}\left(\mathrm{D}_{\mathrm{w}}^{\mathbf{t}} \vartheta\right)(z w) .
$$

Écrivons $w=x_{1} w_{1}+\cdots+x_{g} w_{g}$ avec $x_{i} \in \mathbf{C}$. Pour tout entier $l \geq 0$, la formule de dérivation des fonctions composées donne

$$
\frac{1}{l !} \mathrm{f}^{(l)}(z)=\sum_{|\mathbf{j}|=l}\left(\begin{array}{c}
\mathbf{t}+\mathbf{j} \\
\mathbf{j}
\end{array}\right) x_{1}^{j_{1}} \cdots x_{g}^{j_{g}} \frac{\mathrm{D}_{\mathbf{w}}^{\mathbf{t}+\mathbf{j}} \vartheta}{(\mathbf{t}+\mathbf{j}) !}(z w)
$$

d'où découle la majoration

$$
\max _{\substack{0 \leq h \leq S_{0} \\ 0 \leq \leq \leq(g+t) T}}\left\{\frac{1}{l !}\left|\mathrm{f}^{(l)}(h)\right|\right\} \leq\left(g+\sum_{i=1}^{g}\left|x_{i}\right|\right)^{2(g+t) T} \times \max _{\substack{0 \leq h \leq S_{0} \\|\mathbf{j}| \leq 2(g+t) T}}\left\{\left|\frac{D_{\mathbf{w}}^{\mathbf{j}}}{\mathbf{j} !} \vartheta(h w)\right|\right\} .
$$

D'une part, la somme $\left|x_{1}\right|+\cdots+\left|x_{g}\right|$ est majorée par $\sqrt{g}\|w\|_{\sigma_{0}}$, et nous pouvons utiliser (83) afin de borner la première quantité du membre de droite de 899 . D'autre part, pour $0 \leq h \leq S_{0}$ et $|\mathbf{j}| \leq 2(g+t) T$, la dérivée $\mathrm{D}_{\mathrm{w}}^{\mathbf{j}} \vartheta(h u)$ s'annule et le lemme 5.12 entraîne la majoration

$$
\max _{\substack{0 \leq h \leq S_{0} \\ 0 \leq l \leq(g+t) T}}\left\{\frac{1}{l !}\left|\mathrm{f}^{(l)}(h)\right|\right\} \leq e^{-c_{33} U_{0}}\left(1+\left(S_{0}\left\|u_{0}\right\|_{\sigma}\right)^{2}\right)^{D_{0} / 2}\|s\|_{\infty, \sigma}
$$

avec $c_{33}:=c_{32}-c_{31} \geq 23 g^{2}(g+t)$.

Voici un dernier lemme technique avant de commencer l'extrapolation.

Lemme 5.13. Pour tout entier $m \in\{0, \ldots,(g+t) S\}$, on a

$$
\sup _{\substack{z \in \mathbf{C} \\|z| \leq 4 m e}}\{|\mathrm{f}(z)|\} \leq e^{c_{34} U_{0}}\left(1+\left(m\left\|u_{0}\right\|_{\sigma}\right)^{2}\right)^{D_{0} / 2}\|s\|_{\infty, \sigma}
$$

avec

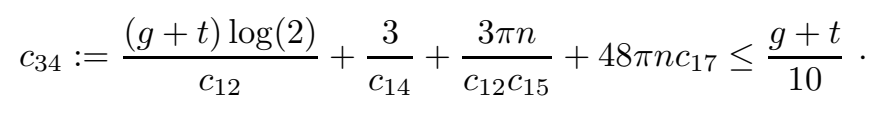

Démonstration. Soit $z \in \mathbf{C},|z| \leq 4 m \mathfrak{e}$. L'inégalité de Cauchy (comme pour (81), avec $r=1 / 2$ ) fournit la majoration

$$
|\mathrm{f}(z)| \leq 2^{|\mathbf{t}|} \sup _{\substack{x \in W_{\sigma} \\\|x\|_{\sigma} \leq 1 / 2}}|\vartheta(z w+x)| \leq 2^{|\mathbf{t}|} \sup _{\substack{x \in t_{\sigma}(\mathbf{C}) \\\|x\|_{\sigma} \leq 1}}|\vartheta(z u+x)|
$$

car $4 m \mathfrak{e}\|u-w\|_{\sigma} \leq 1 / 2$. La formule (78) permet de majorer $|\vartheta(z u+x)|$ en fonction de $\|s\|_{\infty, \sigma}$ et l'on obtient

$$
\begin{aligned}
|\mathrm{f}(z)| \leq & 2^{(g+t) T}\left(1+\left(1+4 m \mathfrak{e}\left\|u_{0}\right\|_{\sigma}\right)^{2}\right)^{D_{0} / 2} \\
& \times \exp \left\{\frac{3 \pi}{2} \sum_{i=1}^{n} D_{i}\left(4 m \mathfrak{e}\left\|u_{i}\right\|_{\sigma}+1\right)^{2}\right\} \times\|s\|_{\infty, \sigma} .
\end{aligned}
$$

Les estimations

(lemme 5.1, (v)) et

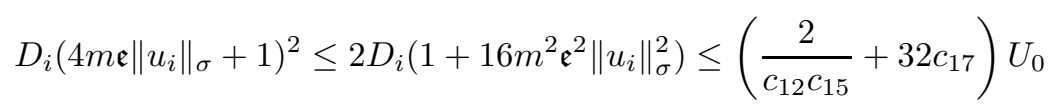

$$
1+\left(1+4 m \mathfrak{e}\left\|u_{0}\right\|_{\sigma}\right)^{2} \leq 1+2\left(1+16 m^{2} \mathfrak{e}^{2}\left\|u_{0}\right\|_{\sigma}^{2}\right) \leq 32 \mathfrak{e}^{2}\left(1+m^{2}\left\|u_{0}\right\|_{\sigma}^{2}\right),
$$

entraînent le lemme 5.13 . 
Les estimations précises de $\left\|\varphi_{i}\right\|_{\sigma}$ mentionnées dans le titre de cette partie repose sur le « lemme de Schwarz approché » suivant.

Lemme 5.14 (Lemme 2 de [18]). Soit $f$ une fonction holomorphe dans le disque centré en 0 et de rayon $R$. Soit $S_{1}$ un entier $\geq 2$. Supposons que $R \geq 2 S_{1}$ et considérons un nombre réel $r \in\left[S_{1}, R / 2\right]$. Soit également $T_{1}$ un entier strictement positif. Alors

$$
\sup _{|z| \leq 2 r}|f(z)| \leq 2\left(\frac{4 r}{R}\right)^{T_{1} S_{1}} \sup _{|z| \leq R}|f(z)|+5\left(\frac{18 r}{S_{1}}\right)^{T_{1} S_{1}} \max _{\substack{0 \leq h \leq S_{1} \\ 0 \leq l \leq T_{1}}}\left\{\frac{1}{l !}\left|f^{(l)}(h)\right|\right\} .
$$

Nous pouvons maintenant énoncer le principal résultat de cette section, qui est au cœur du procédé d'extrapolation de la méthode de Baker.

Proposition 5.15. Pour toute place $\sigma$ de $K$ au-dessus de $\sigma_{0}: k \hookrightarrow \mathbf{C}$ et tout entier $i \in$ $\left\{i_{0}, \ldots, N\right\}$, on $a$

$$
\left\|\varphi_{i}\right\|_{\sigma} \leq e^{-c_{35} U_{0}} \sup \left\{\frac{\|s\|_{\infty, \sigma}}{\|s\|_{\overline{\mathcal{E}}, \sigma}} ; s \in \mathcal{E}_{\sigma} \backslash\{0\}\right\}
$$

avec une constante 035 (définie dans la preuve) $\geq \frac{g+t}{4}$.

Démonstration. Compte tenu des préliminaires, la démonstration de cette proposition est une application directe du lemme 5.14. Nous l'employons avec $R:=4 m \mathfrak{e}$ (notons que $m>S_{0}$ à cause du choix de $\left.i \geq i_{0}\right), T_{1}:=(g+t) T, S_{1}:=S_{0}, r:=m$ et $f$ définie par (88). De cette manière, nous obtenons avec la borne $(90)$ et le lemme 5.13 :

$$
\left|\frac{1}{\mathbf{t} !} \mathrm{D}_{\mathrm{w}}^{\mathbf{t}} \vartheta(m w)\right| \leq e^{-c_{36} U_{0}}\left(1+\left(m\left\|u_{0}\right\|_{\sigma}\right)^{2}\right)^{D_{0} / 2}\|s\|_{\infty, \sigma}
$$

où

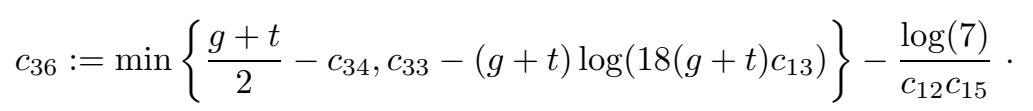

Avec nos choix des paramètres, l'on s'aperçoit que le minimum est obtenu pour $\frac{g+t}{2}-\phi_{34}$ car $18(g+t) \phi_{13} \leq e^{20 g^{2}}$ et donc $c_{33}-(g+t) \log \left(18(g+t) c_{13}\right)>(g+t) / 2$. Une seconde utilisation du lemme 5.12 conduit à la majoration

$$
\left|\frac{1}{\mathbf{t} !} \mathrm{D}_{\mathrm{w}}^{\mathbf{t}} \vartheta(m u)\right| \leq e^{-c_{37} U_{0}}\left(1+\left(m\left\|u_{0}\right\|_{\sigma}\right)^{2}\right)^{D_{0} / 2}\|s\|_{\infty, \sigma}
$$

avec

$$
c_{37}:=\min \left\{c_{36}, c_{32}\right\}-\frac{\log (2)}{c_{12} c_{15}}=c_{36}-\frac{\log (2)}{c_{12} c_{15}} .
$$

La proposition 5.15 découle alors de l'inégalité (80) (en remplaçant $u_{i}^{\prime}$ par $m u_{i}$ ) et la constante que l'on obtient est

$$
435:=\sqrt{37}-\frac{(g+t) \log (g)}{2 \ell_{12}} .
$$

La minoration de 635 est immédiate compte tenu de la borne (91) de

5.11. Fin de la preuve. Résumons la série de résultats que nous avons obtenu jusqu'à présent dans l'énoncé suivant.

Proposition 5.16. Pour tout entier $i \in\{1, \ldots, N\}$, on a

$$
\widehat{\mu}_{\max }\left(\overline{\mathcal{G}}_{i}\right)+h\left(\varphi_{i}\right) \leq c_{38} \frac{U_{0}}{D}+\Xi\left[\left(\mathcal{X}, \overline{\mathcal{M}},\left(\mathrm{d} \mu_{\sigma}\right)_{\sigma: K \hookrightarrow \mathbf{C}}\right)\right]
$$

avec

Pour tout $i \in\left\{i_{0}, \ldots, N\right\}$, on a

$$
c_{38}:=c_{19}+c_{28}+c_{30}+\frac{10 g^{3}(g+t)}{\hbar_{12}} \leq \frac{g+t}{25} .
$$

$$
\widehat{\mu}_{\max }\left(\overline{\mathcal{G}}_{i}\right)+h\left(\varphi_{i}\right) \leq\left(c_{38}-\varphi_{35}\right) \frac{U_{0}}{D}+\Xi\left[\left(\mathcal{X}, \overline{\mathcal{M}},\left(\mathrm{d} \mu_{\sigma}\right)_{\sigma: K \hookrightarrow \mathbf{C}}\right)\right] .
$$


Démonstration. Soit $i \in\{1, \ldots, N\}$. Des propositions 5.8, 5.10 et 5.11, nous déduisons

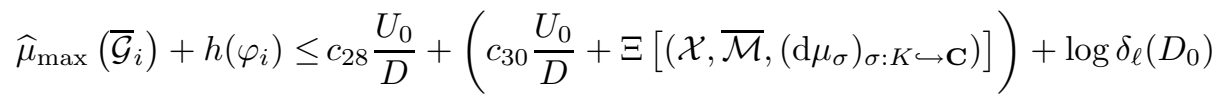

$$
\begin{aligned}
& +\frac{\ell}{[K: \mathbf{Q}]} \sum_{\sigma: K \hookrightarrow \mathbf{C}} \log ^{+} r_{\sigma}\left(m p_{1}, \ldots, m p_{n}\right) \text {. }
\end{aligned}
$$

Grâce à la proposition 4.17 et à la remarque 4.18, (2), nous pouvons majorer le dernier terme (avec la somme) par

$$
\begin{aligned}
2(g+t) \frac{U_{0}}{[12 \mathfrak{a} \log \mathfrak{e}} \times 5 g^{3} \max \left\{1, \mathrm{~h}_{F}\right\} & \left.\leq \frac{10 g^{3}(g+t)}{c \sqrt[2]{2}} \frac{U_{0}}{D} \quad \text { (par définition de } \mathfrak{a}\right) \\
& \leq \frac{1}{1000} \frac{U_{0}}{D} .
\end{aligned}
$$

En ce qui concerne le terme $\log \delta_{\ell}\left(D_{0}\right)$ restant, il est majoré par $2(g+t) T \log \left(4 D_{0}\right)$ d'après l'estimation donnée au début du $\S 5.8$, p. 42. La borne (iv) du lemme 5.1 permet alors d'estimer cette dernière quantité (la constante $d_{19}$ apparaît dans la preuve du lemme 5.1, p. 32) et l'inégalité (92) s'ensuit. La majoration de $c_{38}$ par $(g+t) / 25$ résulte des majorations (déjà vues) $\widehat{c 19} \leq 1 / 50, \phi_{28} \leq(g+t) / 100, \phi_{30} \leq(g+t) / 100$ et de (94). Quant à (93), nous séparons la somme $\sum_{\sigma: K \hookrightarrow \mathbf{C}} \log \left\|\varphi_{i}\right\|_{\sigma}$ en deux parties selon que $\sigma \mid \sigma_{0}$ ou $\sigma \nmid \sigma_{0}$. La première partie est majorée par

$$
-{ }_{35}[K: k] U_{0}+\sum_{\sigma \mid \sigma_{0}} \log \max _{\substack{s \in \mathcal{E}_{\sigma} \\ s \neq 0}}\left\{\frac{\|s\|_{\infty, \sigma}}{\|s\|_{\overline{\mathcal{E}}, \sigma}}\right\}
$$

grâce à la proposition 5.15 et la seconde partie peut être majorée en utilisant la proposition 5.11 . L'inégalité (93) s'obtient alors de la même manière que ci-dessus.

Comme $\varphi$ est une application injective (lemme 5.6) et $E \neq\{0\}$, nous pouvons appliquer l'inégalité de pentes générale du lemme 4.12 sous la forme :

$$
\widehat{\mu}(\overline{\mathcal{E}}) \leq \sum_{i=1}^{N} \frac{\operatorname{dim}\left(E_{i} / E_{i+1}\right)}{\operatorname{dim} E}\left(\widehat{\mu}_{\max }\left(\overline{\mathcal{G}}_{i}\right)+h\left(\varphi_{i}\right)\right),
$$

qui devient après simplification :

$$
\begin{aligned}
\widehat{\mu}(\overline{\mathcal{E}}) \leq & \left(1-\frac{\operatorname{dim} E_{i_{0}}}{\operatorname{dim} E}\right) \max _{1 \leq i \leq i_{0}-1}\left\{\widehat{\mu}_{\max }\left(\overline{\mathcal{G}}_{i}\right)+h\left(\varphi_{i}\right)\right\} \\
& +\frac{\operatorname{dim} E_{i_{0}}}{\operatorname{dim} E} \max _{i_{0} \leq i \leq N}\left\{\widehat{\mu}_{\max }\left(\overline{\mathcal{G}}_{i}\right)+h\left(\varphi_{i}\right)\right\} .
\end{aligned}
$$

Comme $\operatorname{dim} E_{i_{0}} \geq \frac{\operatorname{dim} E}{2}$ (lemme 5.9), la proposition 5.16 entraîne

$$
\widehat{\mu}(\overline{\mathcal{E}})-\Xi\left[\left(\mathcal{X}, \overline{\mathcal{M}},\left(\mathrm{d} \mu_{\sigma}\right)_{\sigma: K \hookrightarrow \mathbf{C})}\right)\right]\left(c_{38}-\frac{\Phi 35}{2}\right) \frac{U_{0}}{D} \leq \frac{(g+t) U_{0}}{D}\left(\frac{1}{25}-\frac{1}{8}\right) .
$$

Ce qui contredit la minoration (66) donnée par la proposition 5.5 (p. 37). Ainsi, l'hypothèse (H) est fausse, ce qui donne la minoration (52) du théorème 5.2 pour la distance de $u$ à $W_{\mathbf{C}}$.

Remarque 5.17. Comme me l'a fait remarqué le rapporteur, le fait que la majoration précise (95) ne soit vraie qu'aux places de $K$ divisant $\sigma_{0}$ est l'unique raison pour laquelle le degré $D=[k: \mathbf{Q}]$ apparait dans la mesure finale. Au lieu d'une seule place $\sigma_{0}$, nous pouvons considérer un sousensemble I de l'ensemble des places archimédiennes de $k$. En séparant la somme $\sum_{\sigma: K \hookrightarrow \mathbf{C}} \log \left\|\varphi_{i}\right\|_{\sigma}$ en les $\sigma$ qui divisent ou non une place de I, nous déduisons de l'inégalité (96) la minoration

$$
\max _{\sigma \in \beth} \log \mathrm{d}_{\sigma}\left(u_{\sigma}, W_{\sigma}\right) \geq-c U_{\beth}
$$

où

(i) $\mathrm{d}_{\sigma}$ est la distance sur $t_{G_{\sigma}}(\mathbf{C})$ induite par $\|\cdot\|_{\sigma}$,

(ii) $u_{\sigma}$ (resp. $\left.u_{i, \sigma}\right)$ est un logarithme de $\sigma(p)$ (resp. $\sigma\left(p_{i}\right)$ ),

(iii) $U_{\beth}$ est le nombre $U$ où le degré $D$ a été remplacé par $D / \operatorname{card} \Xi$ et $\log a_{i}$ doit être plus grand que $\left(\mathfrak{e}\left\|u_{i, \sigma}\right\|_{\sigma}\right)^{2}$ card $\beth / D$ pour tout $\sigma \in \Xi$. 
Il reste à déduire l'inégalité (11) du théorème 3.1 de ce résultat. Rappelons que $U_{0}$ a été choisi comme l'unique nombre réel positif tel que $x(\mathrm{H})=1$. Nous pouvons l'écrire explicitement. Pour $1 \leq i \leq n$, définissons

Alors on a

$$
\mathrm{a}_{i}^{\prime}:=\left(1+\frac{D S_{0}}{\log \mathfrak{e}} \log a_{i}\right)^{-1} .
$$

$$
\begin{aligned}
U_{0}^{\varsigma \mathrm{H}}= & \frac{\mathscr{1 B}_{13} q_{14}^{\operatorname{dim} \mathrm{V}} q_{15}^{\operatorname{dim} \mathrm{B}}}{\phi_{16}} \times \frac{S_{0}}{\left(S_{0} \log \mathfrak{e}\right)^{\varrho_{\mathrm{H}}}}\left(\log (b)+S_{0}^{y} \log \mathfrak{e}\right)^{t-\operatorname{dim} \mathrm{V}} \\
& \times \frac{\mathscr{H}\left(\mathrm{B} ; \mathrm{a}_{1}^{\prime}, \ldots, \mathrm{a}_{n}^{\prime}\right)}{\mathscr{H}\left(A ; \mathrm{a}_{1}^{\prime}, \ldots, \mathrm{a}_{n}^{\prime}\right)} .
\end{aligned}
$$

Comme $\mathrm{a}_{1}^{\prime} \leq \cdots \leq \mathrm{a}_{n}^{\prime}$, nous pouvons majorer $\mathscr{H}\left(\mathrm{B} ; \mathrm{a}_{1}^{\prime}, \ldots, \mathrm{a}_{n}^{\prime}\right)$ par

$$
\left(\operatorname{deg}_{\iota} \mathrm{B}\right)\left\{\prod_{i=\delta_{\mathrm{B}}+1}^{n}\left(\mathrm{a}_{i}^{\prime}\right)^{g_{i}}\right\}\left(\mathrm{a}_{\delta_{\mathrm{B}}}^{\prime}\right)^{\operatorname{dim} \mathrm{B}-\left(g_{\delta_{\mathrm{B}}+1}+\cdots+g_{n}\right)}
$$

car $\delta_{\mathrm{B}}$, défini après (10), p. 9, est le plus grand entier $i \in\{1, \ldots, n\}$ tel que $g_{n}+\cdots+g_{i}>\operatorname{dim} \mathrm{B}$. Nous obtenons alors

avec

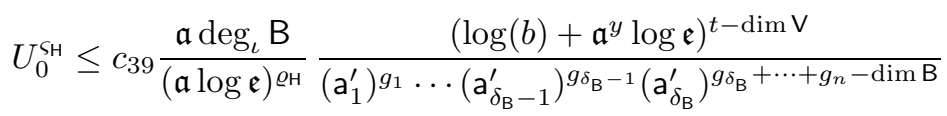

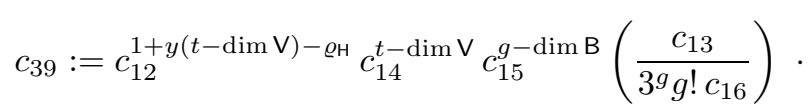

En utilisant $\left(\mathrm{a}_{i}^{\prime}\right)^{-1} \leq 42\left(1+\frac{D \mathfrak{a}}{\log \mathfrak{e}} \log a_{i}\right)$, on a $U_{0}^{\varsigma_{\mathrm{H}}} \leq c_{40} U^{\varsigma_{\mathrm{H}}}$ avec $4:=q_{12}^{9}{ }^{\operatorname{dim} \mathrm{B}}$ [39. Il reste alors à démontrer que $c_{5} \geq \sqrt{20}\left(c_{40}\right)^{1 / \varsigma_{H}}$. Comme $\phi_{15} \geq \phi_{14}$ et $t-\operatorname{dim} \mathrm{V} \geq 1$, on a

Observons alors

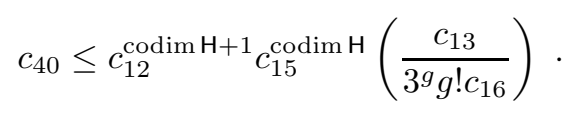

- $\sqrt{12} \leq 10^{4}(g+t)^{6}$ et $\varnothing 2024(g+t)^{3}$ (évident),

- $\sqrt{13} \leq 10^{3 g}(g+t)^{3 g+t}$ (grâce à $2^{t} \leq 2^{g}$ et $\alpha ! \leq \alpha^{\alpha-1}$ pour tout $\alpha \in \mathbf{N}^{*}$ ),

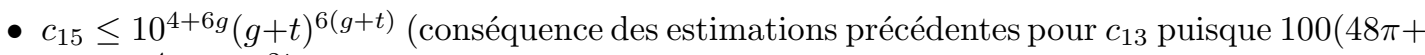
$\left.6) \leq 10^{4}(g+t)^{2 t}\right)$

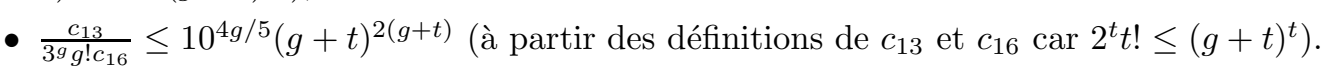

Si nous mettons ces bornes dans $\phi 20(40)^{1 / \varsigma_{H}}$, nous obtenons

$$
\begin{aligned}
& 24 \times 10^{(6 g+8) \frac{\text { codim } H}{\varsigma_{H}}+4(1+g / 5) \frac{1}{\varsigma_{H}}} \\
& \times(g+t)^{3+6 / \varsigma_{H}+6(g+t+1) \frac{\text { codim } H}{\varsigma_{H}}+\frac{2(g+t)}{\varsigma_{H}}} .
\end{aligned}
$$

Cette expression se simplifie si l'on tient compte de $(g+t)$ codim $\mathrm{H} /\left(4 \varsigma_{\mathrm{H}}\right) \geq 1$ qui résulte de (49) en observant

$$
\operatorname{codim} \mathrm{H}=\varsigma_{\mathrm{H}}+g-\operatorname{dim} \mathrm{B}+\varrho_{\mathrm{H}} \geq \varsigma_{\mathrm{H}}+g-\operatorname{dim} \mathrm{B} \geq 2 \varsigma_{\mathrm{H}} .
$$

Le majorant de (99) que l'on obtient de la sorte est

$$
24 \times 10^{(6.4 g+10) \frac{\text { codim } H}{\varsigma_{H}}}(g+t)^{3+\frac{6}{\varsigma_{H}}+(6+6(g+t)) \frac{\operatorname{codim} H}{\varsigma_{H}}+\frac{2(g+t)}{\varsigma_{H}}} .
$$

Cette quantité est inférieure à $c_{5}$ encore en vertu de $g+t \geq 2$ et codim $\mathrm{H} \geq 2 \varsigma_{\mathrm{H}}$ (et donc, par exemple, $\left.3+\frac{6}{\varsigma_{\mathrm{H}}} \leq 9 \leq \frac{9(g+t) \operatorname{codim} \mathrm{H}}{4 \varsigma_{\mathrm{H}}}\right)$.

Remarque 5.18. Si nous remplaçons directement nos choix des $\phi_{12}, \ldots, \phi_{16}$ dans $\phi_{20}\left(c_{10}\right)^{1 / \varsigma_{H}}$, nous pouvons obtenir une meilleure constante $c_{5}$ dans le théorème 3.1. Les calculs ne sont pas simples dans le cas général* et nous allons nous contenter de donner une constante un peu plus précise dans

${ }^{*}$ Pour obtenir une bonne constante absolue, les premières dimensions $g=1,2, \ldots$ et $g \gg 1$ devraient être distinguées. 
le cas des courbes elliptiques. Cependant, dans l'appendice, nous expliquerons comment calculer une telle constante si $g$ et $t$ sont donnés numériquement.

Démonstrations des théorèmes 1.1 et 1.3 et du corollaire 1.9 . Comme nous l'avons dit au $\S 3.3$, tous ces énoncés sont des conséquences du théorème 3.1. Les théorèmes 1.1 et 1.3 résultent des choix $n=y=1, u_{0}=0$ et $\mathrm{H}=\{0\}($ resp. $\mathrm{H}=\{0\} \times B)$. Le corollaire 1.2 est un peu plus subtil. Avec les notations du théorème 1.1, choisissons $W_{0}=\{0\}$. On a alors $t=g$ et $\mathrm{d}\left(u, W_{\mathbf{C}}\right)=\|u\|_{\sigma_{0}}$. D'après la définition de $\check{h}(\S 4.4 .1)$ et la proposition 4.7, p. 15, on a

$$
\check{h}(\{0\})=\widehat{\operatorname{deg}}_{\mathrm{n}} \overline{t_{\mathcal{A}}}=-g \widehat{\mu}\left(\overline{t_{\mathcal{A}}^{\mathrm{v}}}\right)=-h_{F}(A)-\frac{1}{2} \log \mathrm{h}^{0}(A, L)+\frac{g}{2} \log \pi .
$$

En vertu de la minoration (21) de Bost, l'on sait que la hauteur de Faltings de $A$ est minorée par $-g \log (2 \pi) / 2$ et l'on peut donc remplacer $\log b$ par son majorant $2 g D$. Nous fixons également

$$
\log \mathfrak{e}=D \max \left\{1, h_{F}(A)+(1 / 2) \log \mathrm{h}^{0}(A, L), \widehat{h}_{L}(p)\right\}
$$

ce qui entraîne $\log ^{+}(D / \log \mathfrak{e})=0$. Si $\mathfrak{e}\|u\|_{\sigma_{0}} \geq 1$, le corollaire 1.2 est démontré car alors $\log \|u\|_{\sigma_{0}} \geq$ $-\log \mathfrak{e}$. Sinon, majorons $\log a \operatorname{par}(\log \mathfrak{e}) / D$ et $\mathfrak{a}$ par 2 . En particulier, on a $\frac{D \mathfrak{a}}{\log \mathfrak{e}} \log a \leq 2$ et l'inégalité (2) du théorème 1.1 implique la minoration voulue avec $c_{1} 2^{(1 / g)+1} 3(g+1) \leq$ 国.

\section{Cas D'un produit de Courbes elliptiques}

Nous allons détailler dans cette partie le cas particulier où la variété abélienne est un produit de courbes elliptiques (ce qui correspond à $g=n$ ) afin d'effectuer une comparaison avec le résultat déjà connu de David 20]. Nous allons rappeler les notations du $\S 3.1$ en les exprimant de manière aussi « concrète » que possible dans le cas particulier qui nous intéresse ici.

Pour $1 \leq i \leq n$, soit $A_{i}$ une courbe elliptique sur $k$, de section nulle $\varepsilon_{i}:$ Spec $k \rightarrow A_{i}$. L'immersion fermée $\varepsilon_{i}$ définit un diviseur effectif $\Theta_{i}$ et le fibré en droites $L_{i}:=\mathcal{O}_{A_{i}}\left(\Theta_{i}\right)$ associé est ample, symétrique et de degré 1 (polarisation principale canonique). Choisissons une base $1, x, y$ de $\mathrm{H}^{0}\left(A_{i}, L_{i}^{\otimes 3}\right)$ de sorte que $A_{i}$ soit représenté par le modèle de Weiertrass $y^{2}=4 x^{3}-g_{2, i} x-g_{3, i}$, avec $g_{2, i}, g_{3, i} \in \mathcal{O}_{k}$. Notons $e_{i}^{*}:=\frac{\mathrm{d} x}{y}$ la différentielle canonique associée à ce modèle et $e_{i} \in t_{A_{i}}$ le vecteur dual. Pour tout plongement complexe $\sigma: k \hookrightarrow \mathbf{C}$, il existe un réseau $\Lambda_{\sigma}^{(i)}=\mathbf{Z} \omega_{1, \sigma}^{(i)}+\mathbf{Z} \omega_{2, \sigma}^{(i)}$ de $\mathbf{C}$ avec $\tau_{\sigma}^{(i)}:=\omega_{2, \sigma}^{(i)} / \omega_{1, \sigma}^{(i)}$ dans le demi-plan supérieur, tel que l'application $A_{i, \sigma}(\mathbf{C}) \rightarrow \mathbf{C} / \Lambda_{\sigma}^{(i)}$, $q \mapsto \int_{[0, q]} e_{i}^{*} \bmod \Lambda_{\sigma}^{(i)}$ soit un isomorphisme analytique de groupes de Lie complexes. La norme hermitienne

$$
\|\alpha\|_{i, \sigma}^{2}:=\frac{\sqrt{-1}}{2} \int_{A_{i, \sigma}(\mathbf{C})} \alpha \wedge \bar{\alpha}
$$

sur le faisceau différentiel $\omega_{A_{i, \sigma} / \mathbf{C}}^{1}$ induit une polarisation de $\mathbf{C} / \Lambda_{\sigma}^{(i)}$ :

$$
(x, y) \in \mathbf{C}^{2} \longmapsto<x, y>_{i, \sigma}:=\frac{x \bar{y}}{\left|\omega_{1, \sigma}^{(i)}\right|^{2} \operatorname{Im}\left(\tau_{\sigma}^{(i)}\right)} .
$$

Cette polarisation couplée au caractère trivial fournit une donnée d'Appell-Humbert qui détermine le fibré en droites canonique $L_{i, \sigma}$ sur $A_{i, \sigma}$ (voir [5]). On notera que par définition

$$
\left\|e_{i}^{*}\right\|_{i, \sigma}^{2}=\frac{\sqrt{-1}}{2} \int_{\mathbf{C} / \Lambda_{\sigma}^{(i)}} \mathrm{d} z \wedge \mathrm{d} \bar{z}=\left|\omega_{1, \sigma}^{(i)}\right|^{2} \operatorname{Im}\left(\tau_{\sigma}^{(i)}\right) .
$$

Soit $t \in\{1, \ldots, n\}$. Considérons une matrice $\beta=\left(\beta_{i, j}\right)$, à $t$ lignes et $n$ colonnes, à coefficients dans $k$ et de rang maximal. Soit $W_{0}$ le sous-espace vectoriel de $t_{A}$, de codimension $t$, défini par les équations

$$
\forall i \in\{1, \ldots, t\}, \quad \beta_{i, 1} z_{1}+\cdots+\beta_{i, n} z_{n}=0 .
$$

Soit $\mathcal{A}_{i} \rightarrow \operatorname{Spec} \mathcal{O}_{k}$ la composante neutre du modèle de Néron de $A_{i}$ et $\mathcal{A}$ le produit des $\mathcal{A}_{i}$ sur $\mathcal{O}_{k}$. En une place ultramétrique $\mathfrak{p}$ de $k$, le schéma $\mathcal{A}_{i} \times \operatorname{Spec} \mathcal{O}_{\mathfrak{p}}$ est le lieu lisse de l'adhérence schématique dans $\mathbf{P}_{\mathcal{O}_{\mathfrak{p}}}^{2}$ d'un modèle minimal de Weierstrass pour $E_{k_{\mathfrak{p}}}$ ( [6], p. 23). Au moyen d'un changement de coordonnées $(x, y) \rightsquigarrow\left(u^{2} x, u^{3} y\right)$ avec $u \in \mathcal{O}_{\mathfrak{p}}$, le modèle $y^{2}=4 x^{3}-g_{2, i} x-g_{3, i}$ peut être rendu minimal en la place $\mathfrak{p}$ et $u^{-1} e_{i}^{*}$ est alors une $\mathcal{O}_{\mathfrak{p}}$-base de $\omega_{\mathcal{A}_{i, \mathfrak{p}} / \mathcal{O}_{\mathfrak{p}}}$. En particulier la norme p-adique de $e_{i}^{*}$ est plus petite que 1 . Notons $\mathcal{W}_{0}=W_{0} \cap t_{\mathcal{A}}$. Il est possible de donner 
une estimation explicite de la hauteur projective $\breve{h}\left(W_{0}\right)=\widehat{\operatorname{deg}}_{\mathrm{n}} \overline{t_{\mathcal{A}} / \mathcal{W}_{0}}$ de cet espace en termes des coefficients $\beta_{i, j}$, de la manière suivante. Pour $1 \leq i \leq t$, posons $\mathfrak{w}_{i}:=\beta_{i, 1} e_{1}^{*}+\cdots+\beta_{i, n} e_{n}^{*} \in t_{A}^{\vee}$ et soit $\mu_{i}$ l'unique élément de $\left(t_{A} / W_{0}\right)^{v}$ vérifiant la relation $\mu_{i}(x)=\mathfrak{w}_{i}(\widetilde{x})$ où $\widetilde{x} \in t_{A}$ est un relèvement de $x \in t_{A} / W_{0}$. Soit $\mathbf{i}=\left(i_{1}, \ldots, i_{t}\right)$ un $t$-uplet d'entiers tels que $1 \leq i_{1}<\cdots<i_{t} \leq n$ et $I$ l'ensemble de tels uplets. Notons $e_{\mathbf{i}}^{*}:=e_{i_{1}}^{*} \wedge \cdots \wedge e_{i_{t}}^{*} \in \bigwedge^{t} t_{A}^{v}$. Le vecteur

$$
s:=\mu_{1} \wedge \cdots \wedge \mu_{t} \in \operatorname{det}\left(t_{A} / W_{0}\right)^{\vee} \hookrightarrow \bigwedge^{t} t_{A}^{\vee}
$$

s'écrit dans la base des $e_{\mathbf{i}}$ en fonction des mineurs maximaux de $\beta$. Plus précisément, soit $\beta_{\mathbf{i}} \in k$ le mineur maximal de $\beta$ obtenu en ne conservant que les colonnes d'indices $i_{1}, \ldots, i_{t}$. On a $s=$ $\sum_{\mathbf{i} \in I} \pm \beta_{\mathbf{i}} e_{\mathbf{i}}^{*}$ et l'on peut ainsi estimer les normes de $s$ aux différentes places de $k$ :

$$
\begin{aligned}
& \|s\|_{v} \leq \max _{\mathbf{i} \in I}\left\{\left|\beta_{\mathbf{i}}\right|_{v}\right\} \quad \text { si } v \text { est ultramétrique, } \\
& \|s\|_{v}=\left(\sum_{\mathbf{i} \in I}\left|\beta_{\mathbf{i}}\right|_{v}^{2}\left\|e_{\mathbf{i}}^{*}\right\|_{v}^{2}\right)^{1 / 2} \text { si } v \text { est archimédienne. }
\end{aligned}
$$

Dans ce dernier cas, les vecteurs $e_{i}^{*} \otimes_{v} 1,1 \leq i \leq n$, sont orthogonaux et la norme de $e_{\mathbf{i}}^{*}$ est le produit des normes de $e_{i_{m}}^{*}, 1 \leq m \leq t$. Pour $\mathbf{i} \in I$, notons

$$
\left|\omega_{1, v}^{(\mathbf{i})}\right|:=\prod_{m=1}^{t}\left|\omega_{1, v}^{\left(i_{m}\right)}\right| \quad \text { et } \operatorname{Im}\left(\tau_{v}^{(\mathbf{i})}\right):=\prod_{m=1}^{t} \operatorname{Im}\left(\tau_{v}^{\left(i_{m}\right)}\right) .
$$

Par définition même du degré d'Arakelov (p. 13), nous avons

$$
\check{h}\left(W_{0}\right)=-\widehat{\operatorname{deg}}_{\mathrm{n}} \overline{\left(t_{\mathcal{A}} / \mathcal{W}_{0}\right)^{\mathrm{v}}}=\frac{1}{[k: \mathbf{Q}]} \sum_{v \text { place de } k} \log \|s\|_{v}
$$

puis, avec les calculs ci-dessus, nous obtenons

$$
\check{h}\left(W_{0}\right) \leq \frac{1}{[k: \mathbf{Q}]} \log \frac{\prod_{\sigma: k \hookrightarrow \mathbf{C}}\left(\sum_{\mathbf{i} \in I}\left|\beta_{\mathbf{i}}\right|_{\sigma}^{2}\left|\omega_{1, \sigma}^{(\mathbf{i})}\right|^{2} \operatorname{Im}\left(\tau_{\sigma}^{(\mathbf{i})}\right)\right)^{1 / 2}}{\mathrm{~N}_{k \mid \mathbf{Q}}\left(\sum_{\mathbf{i} \in I} \mathcal{O}_{k} \cdot \beta_{\mathbf{i}}\right)}
$$

Au moyen des estimations (triviales),

$$
\left|\beta_{\mathbf{i}}\right|_{v} \leq(t !)^{\epsilon_{v}} \prod_{i=1}^{t} \max _{1 \leq j \leq n}\left\{\left|\beta_{i, j}\right|_{v}\right\}
$$

avec $\epsilon_{v}=0$ si $v$ est ultramétrique et 1 sinon, nous déduisons l'inégalité

$$
\check{h}\left(W_{0}\right) \leq \sum_{i=1}^{t} h_{\text {Weil }}\left(\beta_{i, 1}: \cdots: \beta_{i, n}\right)+t \log (t)+\chi_{1}
$$

où

$$
\chi_{1}:=\frac{1}{[k: \mathbf{Q}]} \log \prod_{\sigma: k \hookrightarrow \mathbf{C}}\left(\sum_{\mathbf{i} \in I}\left|\omega_{1, \sigma}^{(\mathbf{i})}\right|^{2} \operatorname{Im}\left(\tau_{\sigma}^{(\mathbf{i})}\right)\right)^{1 / 2} .
$$

Soit $\sigma_{0}$ un plongement complexe privilégié de $k$. Pour $1 \leq i \leq n$, soit $\wp_{i}$ la fonction de Weierstrass relative au réseau $\Lambda_{\sigma_{0}}^{(i)}$. Le modèle de Weierstrass de la courbe elliptique $A_{i}$ fournit un plongement de celle-ci dans $\mathbf{P}_{k}^{2}$ et l'exponentielle de $A_{i, \sigma_{0}}(\mathbf{C})$ s'identifie (via le choix de la base $e_{i}$ ) à l'application $\mathbf{C} \rightarrow \mathbf{P}^{2}(\mathbf{C}), z \mapsto\left(1: \wp_{i}(z): \wp_{i}^{\prime}(z)\right)$ si $z \notin \Lambda_{\sigma_{0}}^{(i)}$ et $(0: 0: 1)$ sinon. Un point $p_{i} \in A_{i}(k)$ s'identifie à un élément $\gamma_{i}=\left(1: \wp_{i}\left(u_{i}\right): \wp_{i}^{\prime}\left(u_{i}\right)\right) \in \mathbf{P}^{2}(k)$ où nous avons confondu le logarithme $u_{i} \in t_{A_{i, \sigma_{0}}}(\mathbf{C})$ de $p_{i}$ (celui considéré au $\S 3.1$ ) avec sa coordonnée (notée aussi $u_{i}$ ) dans la base $e_{i} \otimes_{\sigma_{0}} 1$ de $t_{A_{i, \sigma_{0}}}(\mathbf{C})$. Soit $u_{0} \in t_{A} / W_{0}$ et $u=\left(u_{0}, u_{1}, \ldots, u_{n}\right) \in\left(t_{A} / W_{0}\right)_{\sigma_{0}} \oplus t_{A_{\sigma}}(\mathbf{C})$.

Avant de formuler une variante elliptique du théorème 3.1, il nous faut écrire la distance $\mathrm{d}\left(u, W_{\mathbf{C}}\right)$ en termes de formes linéaires de logarithmes. Afin de ne pas avoir des expressions trop lourdes à manipuler, nous supposerons que les équations de $W_{0}$ dans la base $\left(e_{1}, \ldots, e_{n}\right)$ sont de la forme

$$
\forall i \in\{1, \ldots, t\}, \quad z_{n-t+i}+\sum_{m=1}^{n-t} \beta_{i, m} z_{m}=0 .
$$


Dans ces conditions, la projection $\lambda: t_{A} \rightarrow t_{A} / W_{0}$ s'écrit en termes de coordonnées

$$
\lambda\left(x_{1} e_{1}+\cdots+x_{n} e_{n}\right)=\sum_{i=1}^{t}\left(x_{n-t+i}+\sum_{m=1}^{n-t} \beta_{i, m} x_{m}\right) \lambda\left(e_{n-t+i}\right) .
$$

Notons $\left(u_{0}^{(i)}\right)_{1 \leq i \leq t} \in k^{t}$ les coordonnées du vecteur $-u_{0}$ dans la base $\left(\lambda\left(e_{n-t+i}\right)\right)_{1 \leq i \leq t}$. Soit

$$
\Lambda_{i}:=u_{0}^{(i)}+u_{n-t+i}+\sum_{m=1}^{n-t} \beta_{i, m} u_{m} \in \mathbf{C} .
$$

De la formule (101), nous déduisons les majorations

$$
\begin{aligned}
\mathrm{d}\left(u, W_{\mathbf{C}}\right) & \leq\left\|u_{0}-\lambda\left(u_{1} e_{1}+\cdots+u_{n} e_{n}\right)\right\|_{\overline{t_{\mathcal{A}} / \mathcal{W}_{0}}, \sigma_{0}} \\
& \leq \max _{1 \leq i \leq t}\left\{\left|\Lambda_{i}\right|\right\} \times\left(\sum_{i=1}^{t}\left\|e_{n-t+i}\right\|_{\bar{t}_{\mathcal{A}_{n-t+i}}, \sigma_{0}}\right) .
\end{aligned}
$$

Lorsque $W_{0}$ est un hyperplan $\beta_{1} z_{1}+\cdots+\beta_{n} z_{n}=0$, on a la formule exacte $\left(\right.$ si $\left.\beta_{n} \neq 0\right)$ :

$$
\mathrm{d}\left(u, W_{\mathbf{C}}\right)=\frac{\left|\beta_{n} u_{0}+\beta_{1} u_{1}+\cdots+\beta_{n} u_{n}\right|}{\sqrt{\sum_{i=1}^{n}\left|\omega_{1, \sigma_{0}}^{(i)}\right|^{2} \operatorname{Im}\left(\tau_{\sigma_{0}}^{(i)}\right)\left|\beta_{i}\right|^{2}}}
$$

obtenue par exemple au moyen du vecteur $\sum_{i} \frac{\overline{\beta_{i}}}{\left\|e_{i}\right\|_{\sigma_{0}}^{2}} e_{i}$, orthogonal à $W_{0}$. Notons également que la hauteur $h \overline{\mathcal{O}_{X_{0}}(1)}\left(m u_{0}\right)$ de $m u_{0}$ s'évalue au moyen des coordonnées $u_{0}^{(i)}$ et qu'elle est majorée par :

$$
\frac{1}{[k: \mathbf{Q}]} \log \frac{\prod_{\sigma: k \hookrightarrow \mathbf{C}}\left(1+\sum_{i=1}^{t}\left|m u_{0}^{(i)}\right|_{\sigma}^{2}\left\|\lambda\left(e_{n-t+i}\right)\right\|_{\frac{2}{t_{\mathcal{A}} / \mathcal{W}_{0}}, \sigma}\right)^{1 / 2}}{N_{k \mid \mathbf{Q}}\left(\mathcal{O}_{k}+\mathcal{O}_{k} m u_{0}^{(1)}+\cdots+\mathcal{O}_{k} m u_{0}^{(t)}\right)}
$$

(voir p. 17) et, par conséquent,

$$
\begin{aligned}
h_{\overline{\mathcal{O}_{X_{0}}(1)}}\left(m u_{0}\right) & \leq h_{\text {Weil }}\left(1: m u_{0}^{(1)}: \cdots: m u_{0}^{(t)}\right)+\chi_{2} \\
& \leq h_{\text {Weil }}\left(1: u_{0}^{(1)}: \cdots: u_{0}^{(t)}\right)+\log m+\chi_{2}
\end{aligned}
$$

où

$$
\chi_{2}:=\frac{1}{[k: \mathbf{Q}]} \log \prod_{\sigma}\left(1+\sum_{i=1}^{t} \frac{1}{\left|\omega_{1, \sigma}^{(i)}\right|^{2} \operatorname{Im} \tau_{\sigma}^{(i)}}\right)^{1 / 2},
$$

en majorant $\left\|\lambda\left(e_{n-t+i}\right)\right\|_{\overline{t_{\mathcal{A}} / \mathcal{W}_{0}}, \sigma}$ par $\left\|e_{n-t+i}\right\|_{\overline{t_{\mathcal{A}}}, \sigma}$.

Nous sommes maintenant en mesure d'énoncer une première variante, légèrement simplifiée (et affaiblie), du théorème 3.1 dans ce cadre elliptique.

Théorème 6.1. Avec les notations ci-dessus, considérons également des nombres réels $\mathfrak{e} \geq e$ et $a_{1} \geq \cdots \geq a_{n}$ vérifiant les conditions

$$
\forall i \in\{1, \ldots, n\}, \quad \log a_{i} \geq \max \left\{\widehat{h}\left(\gamma_{i}\right), \frac{\mathfrak{e}^{2}\left|u_{i}\right|^{2}}{D\left|\omega_{1, \sigma_{0}}^{(i)}\right|^{2} \operatorname{Im} \tau_{\sigma_{0}}^{(i)}}\right\}
$$

(où $\widehat{h}\left(\gamma_{i}\right)$ est la hauteur de Néron-Tate de $\left.\gamma_{i}\right)$. Définissons l'entier

$$
\mathfrak{a}:=\left[\frac{D}{\log \mathfrak{e}} \max \left\{1, \log ^{+}\left(\frac{D}{\log \mathfrak{e}}\right), \mathrm{h}_{F}, \log ^{+} \log a_{1}\right\}\right]+1
$$

où, ici, $\mathrm{h}_{F}=\max _{1 \leq i \leq n}\left\{h_{F}\left(A_{i}\right)\right\}$. Soit $b>0$ tel que

$$
\begin{aligned}
& \log b \geq D \sum_{i=1}^{t} h_{\text {Weil }}\left(\beta_{i, 1}: \cdots: \beta_{i, n}\right)+D h_{\text {Weil }}\left(1: u_{0}^{(1)}: \cdots: u_{0}^{(t)}\right) \\
& \quad+D\left(\chi_{1}+\chi_{2}\right)+D \log \mathfrak{a}+20 n^{2}
\end{aligned}
$$


Si $\left(\gamma_{1}, \ldots, \gamma_{n}\right)$ n'est pas un point de torsion modulo les sous-variétés abéliennes strictes de $A_{1} \times$ $\cdots \times A_{n}$ alors, pour toute sous-variété abélienne $B$ de $A$ telle que $W_{0}+t_{B} \neq t_{A}$, on a

$$
\begin{aligned}
& \log \max _{1 \leq i \leq t}\left\{\left|\Lambda_{i}\right|\right\} \\
& \geq-c_{41}(\log b+\mathfrak{a} \log \mathfrak{e})\left((\mathfrak{a} \operatorname{deg} B) \prod_{j=1}^{\operatorname{codim} B}\left(1+\frac{D \mathfrak{a}}{\log \mathfrak{e}} \log a_{j}\right)\right)^{\frac{1}{n-\operatorname{dim}\left(W_{0}+t_{B}\right)}}
\end{aligned}
$$

avec

$$
\left.c_{41}:=(11(n+t))^{13(n+t)\left(1+\frac{\operatorname{codim} B}{n-\operatorname{dim}\left(W_{0}+t B\right.}\right)}\right) .
$$

Lorsqu'il y a une seule forme linéaire (comme dans l'énoncé de David [20]), nous avons l'énoncé suivant, un peu plus précis.

Théorème 6.2. Avec les notations et hypothèses du théorème précédent et si $W_{0}$ est un hyperplan $(t=1)$ alors, pour toute sous-variété abélienne $B$ de $A$ telle que $t_{B} \subseteq W_{0}$, on a

$$
\begin{aligned}
& \log \left|u_{0}+\beta_{1} u_{1}+\cdots+\beta_{n-1} u_{n-1}+u_{n}\right| \\
& \geq-c_{42}(\mathfrak{a} \operatorname{deg} B) \log (\mathfrak{e} b) \prod_{i=1}^{\operatorname{codim} B}\left(1+\frac{D \mathfrak{a}}{\log \mathfrak{e}} \log a_{i}\right)
\end{aligned}
$$

avec

$$
\begin{aligned}
c_{42}:= & 2^{6 n^{2}+16 n+7} 3^{6 n^{2}+3 n} 5^{3 n+3}(n+1)^{6 n^{2}+13 n+11} \\
& \times\left(\frac{1}{2^{6 n+14} 3^{6 n} 5^{3}(n+1)^{6 n+10}}\right)^{\operatorname{dim} B} .
\end{aligned}
$$

Remarque : Ce dernier théorème reste vrai si l'on suppose seulement $u_{0} \neq 0$, sans la condition sur le point $\left(\gamma_{1}, \ldots, \gamma_{n}\right)$.

Démonstrations des théorèmes 6.1 et 6.8. L'hypothèse faite sur le point $\left(\gamma_{1}, \ldots, \gamma_{n}\right)$ est plus forte que l'hypothèse (1) sur le point $p, \mathrm{p} .9$. Nous pouvons donc appliquer le théorème 3.1 aux données du théorème 6.1, en choisissant de plus $\mathrm{H}=\lambda\left(t_{B}\right) \times B$. On a $\varsigma_{\mathrm{H}}=n-\operatorname{dim}\left(W_{0}+t_{B}\right)>0, \delta_{B}=\operatorname{codim} B$ et $\varrho_{\mathbf{H}}=0$. Compte tenu de la comparaison entre $\mathrm{d}\left(u, W_{\mathbf{C}}\right)$ et $\max _{1 \leq i \leq t}\left\{\left|\Lambda_{i}\right|\right\}$ :

$$
\mathrm{d}\left(u, W_{\mathbf{C}}\right) \leq \max _{1 \leq i \leq t}\left\{\left|\Lambda_{i}\right|\right\} b,
$$

(qui découle de (102) et de la définition de $\chi_{2}$ ), l'inégalité (105) résulte de l'inégalité (11) du théorème 3.1 avec une constante $c_{5}+1 \leq c_{41}$. Mentionnons que le $20 n^{2}$ qui est dans le minorant de $\log b$ est un majorant très large de la quantité $\log \left((g+t) \sqrt{12}\left(h_{13}\right)\right.$, qui intervient dans le minorant originel (8) (p. 9) après avoir utilisé la majoration $(104)$ de $h \overline{\mathcal{O}_{X_{0}}(1)}\left(m u_{0}\right)$ donnée ci-dessus. Pour le théorème 6.2, on a de plus $t=\varsigma_{\mathrm{H}}=1$ et l'hypothèse (2) sur le point $p$ est satisfaite dans ce cas (ce qui est également vrai si $u_{0} \neq 0$ sans hypothèse sur $\left.\left(\gamma_{1}, \ldots, \gamma_{n}\right)\right)$. L'on peut donc prendre $y=0$ et le théorème 6.2 découle du théorème 3.1 comme ci-dessus, sauf pour la constante $c_{42}$ dont le calcul est effectué ci-après, dans la seconde partie de l'appendice (p. 57).

Nous retrouvons ainsi les principales caractéristiques décrites dans 28] (et aussi mentionnées dans l'introduction de ce texte) à savoir, une estimation optimale en la hauteur log $b$ du sous-espace $W_{0}$ (il n'y a pas de $\log \log b$ ) et une meilleure dépendance en la hauteur $\log \left(a_{1} \cdots a_{n}\right)$ des points considérés (polynomiale de degré $n+\varepsilon$ au lieu de $n+1+\varepsilon$ dans le travail de David). Pour analyser cela de manière plus précise, nous allons écrire explicitement la mesure obtenue par David [20] en renormalisant ses notations pour les rendre compatibles avec les nôtres. Pour simplifier la discussion nous prenons $\mathfrak{e}=e$. Posons $\Lambda:=u_{0}+\beta_{1} u_{1}+\cdots+\beta_{n-1} u_{n-1}+u_{n}$. Notons comme ci-dessus $\mathrm{h}_{F}=\max _{1 \leq i \leq n}\left\{h_{F}\left(A_{i}\right)\right\}$, quantité qui correspond au $h=\max _{i}\left\{1, h_{\text {Weil }}\left(1: g_{2, i}: g_{3, i}\right), h\left(j_{A_{i}}\right)\right\} \mathrm{du}$ texte de David. Soit $b>0$ tel que

$$
\log b \geq D\left(h_{\text {Weil }}\left(u_{0}\right)+h_{\text {Weil }}\left(\beta_{1}\right)+\cdots+h_{\text {Weil }}\left(\beta_{n-1}\right)\right)+D\left(\chi_{1}+\chi_{2}\right)+D h_{F}+1
$$

(ce n'est pas tout à fait le $\log b$ du théorème 6.1 car il n'y a plus de $D \log \mathfrak{a}$, quantité que nous avons directement reportée dans la mesure qui suit). La mesure du théorème 6.2 s'écrit (en choisissant 
$B=\{0\}):$

$$
\begin{aligned}
\log |\Lambda| & \geq-c_{43}(\log b+D \log \mathfrak{a}) \mathfrak{a} \prod_{i=1}^{n}\left(1+D \mathfrak{a} \log a_{i}\right) \\
& \geq-c_{43}^{\prime} D^{n}\left(\log b+D \log D+D \log ^{+} \log ^{+} \log a_{1}\right) \mathfrak{a}^{n+1} \prod_{i=1}^{n}\left(1+\log a_{i}\right)
\end{aligned}
$$

avec $\log c_{43}=\log c_{43}^{\prime}=6 n^{2} \log n+o\left(n^{2} \log n\right)$. La mesure du théorème 2.1 de [20] est quant à elle :

$$
\begin{aligned}
\log |\Lambda| \geq- & c_{44} D^{n}\left(\log b+D \log D+\log a_{1}\right)(\mathfrak{a}+D \log \log b)^{n+1} \\
\times & \prod_{i=1}^{n}\left(1+\log a_{i}\right)
\end{aligned}
$$

avec $\log c_{44}=2 n^{2} \log n+o\left(n^{2} \log n\right)$. Nous constatons donc bien la disparition de $\log \log b$ et la transformation de $\log a_{1}$ en $D \log ^{+} \log ^{+} \log a_{1}$. La dépendance en les autres paramètres $D, n, \mathrm{~h}_{F}$ est sensiblement la même dans les deux mesures. Enfin, nous avons $\log c_{43}=3 \log c_{44}+o\left(n^{2} \log n\right)$ et nous ne sommes pas parvenu à supprimer ce coefficient 3 . Les raisons sont expliquées à la fin de l'appendice.

Justification de l'inégalité (108). L'obtention de cette inégalité à partir de celle du théorème 2.1 de [20] s'effectue de la manière suivante. Avec les notations de [20], prenons $D \log B=\log \left(b a_{1}\right)$ et $\log V_{i}=\log a_{i}+1$. Ce dernier choix pour $\log V_{i}$ est rendu possible grâce à la troisième remarque page 11 de [20], qui permet de supprimer la hauteur $\mathrm{h}_{F}$ dans le minorant de $\log V_{i}$ lorsque $\mathfrak{e}=e$. La quantité $\log \log (B)+h+\log D$ est alors naturellement majorée (à une constante numérique près) par $\mathfrak{a} / D+\log \log b$ et l'inégalité (108) s'ensuit. Notons que les constantes numériques qui interviennent dans cette transcription ne modifient la constante $C$ de [20] que par un terme exponentiel en $n$, ce qui assure $\log c_{44}=\log C+o\left(n^{2} \log n\right)$.

Remarque 6.3. Dans le texte [20], il n'y a pas la quantité $\chi_{1}+\chi_{2}$ comme dans le minorant de $\log b$ ci-dessus. Mais cette dernière semble être de l'ordre de $h_{F}$ et elle ne joue probablement aucun rôle significatif.

\section{APPEndice : À PRopos DES CONSTANTES NUMÉRIQUes}

Au cours de la démonstration du théorème 5.2, nous avons écrit les différentes conditions que doivent satisfaire les paramètres. En dernier lieu, il est possible de conclure dès lors que 035 $2\left(c_{26}+c_{38}\right)$. Or d'après la définition de $\phi_{55}$, cette condition est remplie lorsque l'on a à la fois

et

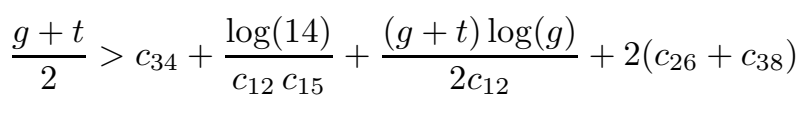

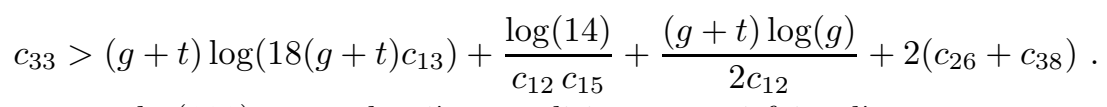

Si l'on tient compte de (109), cette dernière condition est satisfaite dès que

$$
c_{33} \geq \frac{g+t}{2}-c_{33}+(g+t) \log \left(18(g+t) c_{13}\right) .
$$

En revenant aux définitions des constantes $c_{33}$ et ${ }_{34}$, nous constatons que cette inégalité est automatiquement satisfaite si l'on choisit la constante $\alpha_{20}$ assez grande, à savoir

$$
\text { 尼 } \geq \frac{g+t}{2}+\frac{11(g+t)}{\text { 回 }} \log (g+t)+(g+t) \log (18(g+t) \text { 国 }) \text {. }
$$

L'hypothèse (84) est alors vraie. Il n'y a aucune autre condition pour 200 , et nous avons ainsi intérêt à choisir l'égalité dans (111). Compte tenu de cette remarque et en supprimant quelques conditions redondantes, nous pouvons formuler le résultat suivant.

Proposition A.1. Supposons que les six conditions suivantes soient satisfaites.

(1) $q_{12}, \ldots, c$ 的 sont des entiers strictement positifs,

(2) 4 迆 $\geq 18^{g}\left(\frac{(g+t) !}{t !}\right)^{2}$, 
(3) $\sqrt{14} \geq 2 \times 18^{g}\left(\frac{(g+t) !}{t !}\right)^{2}$,

(4) $16 \geq 12^{g} \frac{(g+t) !}{t !}$,

(5) $\frac{\text { c }}{\text { cal }} \geq 3^{g} 2^{g+t} t ! g !(2(g+t)+1)^{g}(g+t)^{2}$,

(6)

$$
\begin{aligned}
& \frac{g+t}{2}>\frac{1}{612}\left(\frac{(g+t) \log (4 g)}{2}+4(g+1)(4 g+2)\right) \\
& +\frac{1}{\sqrt{12}}\left(60 g^{5}(g+t) n+20 g^{3}(g+t)\right)+4(g+1) g \frac{\log \left(1+c_{12}\right)}{c_{12}}
\end{aligned}
$$

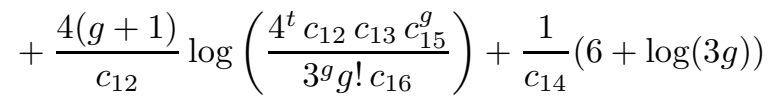

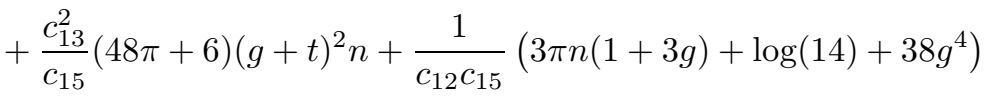

Alors on a

$$
\begin{aligned}
& \log \mathrm{d}(u, W) \geq \\
& -\phi_{20}\left(c_{12}^{1-1} y(t-\operatorname{dim} \mathrm{V})-\varrho_{\mathrm{H}}+g-\operatorname{dim} \mathrm{B} c_{14}^{\operatorname{dim} \mathrm{V}} q_{15}^{\operatorname{dim} \mathrm{B}}\left(\frac{\phi_{13}}{3^{g} g ! \phi_{16}}\right)\right)^{1 / \varsigma_{H}} U .
\end{aligned}
$$

Démonstration. La première condition n'est pas absolument nécessaire mais elle simplifie quelques calculs intermédiaires. Les deuxième et troisième conditions correspondent à $\phi_{15}>q_{21} c_{23}$ et $\phi_{14}>$ $2 c_{21} c_{23}$ provenant de (62), p. 35. En effet, on a

$$
\begin{aligned}
\text { 221 } c_{23} & =6^{g} \frac{(g+t) !\left(\operatorname{dim} G_{\kappa}^{\star}\right) ! 3^{g_{1}+\cdots+g_{\kappa-1}}\left(g^{\star}-\operatorname{dim} \pi_{\kappa}\left(A^{\star}\right)\right) !}{t !\left(t^{\star}+g^{\star}\right) !} \\
& <18^{g} \frac{(g+t) !}{t !} \frac{\left(g+t^{\star}\right) !}{t^{\star} !} \frac{t^{\star} !\left(g^{\star}-\operatorname{dim} \pi_{\kappa}\left(A^{\star}\right)\right) !}{\left(t^{\star}+g^{\star}\right) !}
\end{aligned}
$$

car $\operatorname{dim} G_{\kappa}^{\star} \leq g+t^{\star}$ et donc $c$ 2n $c_{23} \leq 18^{g}\left(\frac{(g+t) !}{t !}\right)^{2}$. La condition (4) de la proposition A.1 est on $>4^{g} c_{23} c_{25}$, qui se trouve à la fin de la preuve de la proposition 5.3, p. 36, car

$$
c_{23} c_{25}=3^{g_{1}+\cdots+g_{\kappa-1}} \frac{(g+t) !}{t !} \frac{\left(\operatorname{dim} A_{\kappa}^{\star}\right) !}{g !} \frac{t^{\star} !\left(g^{\star}-\operatorname{dim} \pi_{\kappa}\left(A^{\star}\right)\right) !}{\left(t^{\star}+g^{\star}\right) !}
$$

est strictement inférieur à $3^{g}(g+t) ! / t$ !. La condition (5) provient de la condition de Siegel (73), p. 42. Quant à la dernière, il s'agit précisément de l'inégalité de pentes qui résume toutes les estimations obtenues dans la preuve du théorème 5.2. C'est en réalité l'inégalité (109) réécrite en termes seulement des constantes $-\sqrt{20}\left(c_{40}\right)^{1 / \varsigma_{H}} U$ que l'on trouve à la toute fin de la preuve (p. 51).

Il ressort clairement de cette proposition que les constantes $\Upsilon_{12}, \ldots, h_{16}$ doivent être choisies aussi petites que possible. Notre choix a été effectué dans l'ordre suivant : $\alpha_{16}, \phi_{14}, \phi_{13}, \phi_{15}$ (avec la condition (112), qui implique (2)). Il reste alors une seule condition provenant de (112) qui concerne 12. Ainsi, numériquement, les constantes $h_{16}, \phi_{14}, \phi_{13}$ étant fixées (et les choix sont presque les meilleurs possibles), il est facile de choisir au mieux of $d_{12}$ et $\phi_{15}$ de sorte que les conditions (2) et (112) soient remplies, et l'on obtient alors des constantes numériques très précises (éventuellement à l'aide de calculs sur machine).

Pour finir, mettons en œuvre ces quelques règles dans le cas elliptique afin d'obtenir la constante $c_{42}$ du théorème 6.2. Nous revenons aux hypothèses de ce théorème, et, en particulier, on a $g=n$, $t=1$. Nous choisissons

$$
\begin{aligned}
& 016=12^{n}(n+1) \text { ! } \\
& \text { 14 }=2^{n+1} 3^{2 n}(n+1) !^{2} \\
& \text { 运 }=2^{3 n+1} 3^{2 n}(n+1) !^{2}(2 n+3)^{n}(n+1) \\
& 6=4(n+1)^{2}(48 \pi+6) d_{13}^{2}
\end{aligned}
$$


À l'aide d'un calcul direct, nous déduisons que l'inégalité (112) est satisfaite si

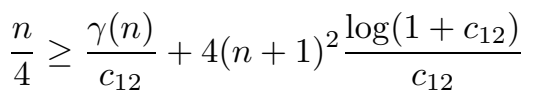

où

$$
\begin{aligned}
\gamma(n):= & (n+1) \frac{\log (4 n)}{2}+8(n+1)(2 n+1) \\
& +60 n^{6}(n+1)+20 n^{3}(n+1)+4(n+1) \log (4) \\
& +4(n+1) \log \left(2^{n+1}(2 n+3)^{n}(n+1)^{2}\right)+4(n+1) n \log (\text { 回 }) .
\end{aligned}
$$

Observons que $\gamma(n) \leq 145 n^{5}(n+1)^{2}$ car cette inégalité est vraie pour $n=1(\gamma(1) \simeq 456,71)$ et la fonction $n \mapsto\left(\gamma(n)-60 n^{6}(n+1)\right) /\left(n^{5}(n+1)^{2}\right)$ est une somme de fonctions décroissantes $\left(n \in \mathbf{N}^{*}\right)$. Montrons alors que $12=800 n^{4}(n+1)^{2}$ convient. En effet, avec nos choix, on a

$$
\frac{\gamma(n)}{\sqrt{12}}+4(n+1)^{2} \frac{\log (1+412)}{d .2} \leq \frac{n}{5}+\frac{\log 801+4 \log (n)+2 \log (n+1)}{200 n^{4}} \text {. }
$$

Le dernier terme est une fonction décroissante de $n$, donc majoré par sa valeur en $n=1$, ce qui implique

$$
\frac{\gamma(n)}{d_{12}}+4(n+1)^{2} \frac{\log (1+\sqrt{12})}{\gamma_{12}} \leq \frac{n}{5}+0.0404<\frac{n}{4} .
$$

La proposition A.1, la fin de la preuve du théorème 6.2 et la remarque sur col qui suit (111) impliquent que nous pouvons choisir un majorant quelconque de

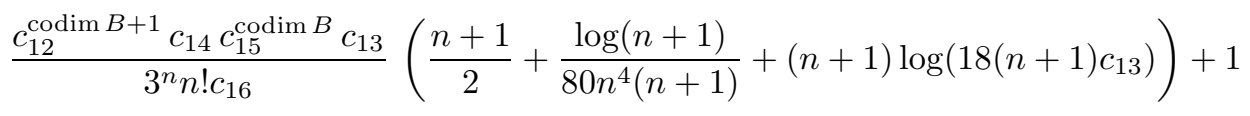

pour $c_{42}$. Comme $(n+1) ! \leq(n+1)^{n}$, on a

$$
\text { 通 } \leq 2^{3 n+1} 3^{3 n}(n+1)^{3 n+1}
$$

donc $18(n+1)$ 目 $\leq(6(n+1))^{3 n+2} \leq 6^{3(n+1)} e^{\frac{3}{2}(n+1)^{2}}$ et le terme entre parenthèses de (115) est borné par $5(n+1)^{3}$,

$$
\begin{aligned}
& \text { 回 } \leq 2^{n+1} 3^{2 n}(n+1)^{2 n}, \\
& \text { 㴘 } \leq 2^{6 n+9} 3^{6 n} 5(n+1)^{6 n+4}\left(\operatorname{car} 4(48 \pi+6) \leq 2^{7} \times 5\right) .
\end{aligned}
$$

Avec en outre $12 \leq 2^{5} 5^{2}(n+1)^{6}$ et

$$
\frac{d[3}{3^{n} n ! \text { 迫 }}=2^{n+1}(2 n+3)^{n}(n+1)^{2} \leq 2^{n+1} 3^{n}(n+1)^{n+2}
$$

un calcul direct fournit la valeur que nous avons donnée à $c_{42}$.

Compléments . * Il est impossible ici d'affaiblir le terme $(n+1)^{6 n^{2}}$ qui est dans $c_{42}$ en $(n+1)^{\epsilon n^{2}}$ avec $\epsilon<6$, contrairement au résultat de David qui autorise $\epsilon=2$. En réalité, dans le cas général, on montre que

$$
c_{5} \gg g^{2 g \frac{\operatorname{codim} H}{\varsigma_{H}}+4 g \frac{g-\operatorname{dim} B}{\varsigma_{H}}}
$$

car nécessairement $d$ 国 $\gg g^{2 g}$ et 1 国 $\gg g^{6 g}$. Il semble qu'au moins deux raisons se cachent derrière cela. La première provient de la condition (1) de la proposition A.1, qui implique $g \log (g)=$ $O(\log$ (1, $)$, tandis qu'il y avait seulement $g=O(\log c$ 国 $)$ dans op. cit. Cette condition provient de l'introduction du sous-groupe auxiliaire $G_{\kappa}^{\star}$ dans la preuve de la propriété d'injectivité de $\varphi$ (p. 35), sous-groupe dont nous avons eu besoin pour prendre en compte la possibilité que certains des paramètres $D_{i}$ soient nuls. La seconde raison est que le passage de $\mathscr{H}\left(G ; D_{0}^{\prime}, \ldots, D_{n}^{\prime}\right)$ à $\operatorname{rg}(\mathcal{E})$ dans la preuve du lemme 5.9 (inégalité (72), p. 41) crée un facteur $(g+t)$ ! qui apparaît dans 2.9 et qui se répercute ensuite dans la condition (5) de la proposition A.1. Je ne sais pas comment résoudre ces problèmes techniques. Toutefois, dans la pratique, l'on peut imaginer les contourner au moyen du groupe $\mathrm{H}$. En effet, il ressort de l'inégalité (113) ainsi que la valeur de la constante $c_{5} \mathrm{du}$ théorème 3.1 que la constante finale est d'autant plus petite que le rapport

$$
\frac{\operatorname{codim} \mathrm{H}}{\varsigma_{\mathrm{H}}}=\frac{\operatorname{codim} \mathrm{H}}{t-\operatorname{dim}\left(\mathrm{V}+\lambda\left(t_{\mathrm{B}}\right)\right)}=\frac{\operatorname{codim} \mathrm{H}}{\operatorname{codim} \mathrm{H}-\operatorname{codim}_{W_{\bar{k}}}\left(W_{\bar{k}} \cap t_{\mathrm{H}}\right)}
$$


l'est. Un choix adéquat de $\mathrm{H}$ permet donc de diminuer la constante quitte à affaiblir l'aspect théorique de la mesure d'indépendance linéaire de logarithmes, à travers le paramètre $U$ (voir la discussion qui suit le théorème 3.1). Par exemple, la constante $c_{4}$ du théorème 1.3 est « seulement 》 exponentielle en $g \log g$, et non, comme à l'accoutumée, exponentielle en $g^{2} \log g$. Or la taille de cette constante est un obstacle majeur lorsque l'on cherche à résoudre des équations diophantiennes à l'aide de telles mesures d'indépendances linéaires de logarithmes abéliens. Il existe des procédés fondés sur l'algorithme LLL qui permettent (au moyen de calculs avec des ordinateurs) de réduire drastiquement ce type de constantes et, par suite, le nombre de solutions potentielles de l'équation diophantienne en question (voir [65, 30, 66]). Néanmoins ces méthodes restent subordonnées à une limitation physique simple : la constante initiale doit être de taille « raisonnable » pour être codée informatiquement. Dans ces applications, la variété abélienne choisie est souvent la puissance $r$ ème d'une courbe elliptique $A$ où $r$ est le rang de Mordell-Weil de $A(k)$. Avoir ainsi la possibilité de prendre la racine $(r+1)^{\text {ème }}$ de la constante « usuelle » peut s'avérer primordial, ou, à tout le moins, plus important que la qualité de la mesure en les paramètres $\log b$ et $\log a_{i}, 1 \leq i \leq n$, comme les experts le savent bien.

* Il serait intéressant de supprimer la quantité $\log ^{+} \log a_{1}$ dans la définition de $\mathfrak{a}$ (page 9). C'est une question a priori délicate qui n'est toujours pas résolue même dans le cas d'un groupe linéaire, sauf si l'on impose une condition de rationalité supplémentaire sur $W_{0}$, à savoir $W_{0}$ est l'espace tangent d'une sous-variété abélienne de $A$. Une étude systématique de cette question comportant des rappels historiques est faite dans le texte [29].

* L'hypothèse (1) ou (2) sur le point $p$ dans le théorème 3.1 est la conséquence d'une complication technique qui est apparue au cours de la démonstration et que nous n'avons pu surmonter qu'avec cette hypothèse. Elle résulte de la nécessité de pouvoir extrapoler sur les multiples entiers $2 p, 3 p, \ldots$. du point $p$ afin de se placer dans le cadre «strict » de la méthode de Baker. Ces multiples doivent donc être distincts modulo les sous-variétés abéliennes propres de $A$. Il s'agit de l'hypothèse qui permet d'être dans le « cas non-périodique » de la preuve classique. Pourtant, P. Philippon \& M. Waldschmidt avaient montré comment l'on pouvait s'affranchir d'une telle hypothèse (autrement dit, ils ont su résoudre le « cas périodique ») en effectuant une « extrapolation sur les dérivations » le long de $W_{0}$ (à la Gel'fond) lorsque $p$ est de torsion modulo une sous-variété abélienne propre de A. À la suite du changement complet de la structure de la démonstration présentée ici, nous ne sommes pas parvenu à incorporer leur idée dans notre preuve.

\section{RÉFÉRENCES}

[1] A. BAKER. Linear forms in the logarithms of algebraic numbers. I, II, III. Mathematika 13 :204-216, 1966; ibid. $14: 102-107,1967$; ibid. $14: 220-228,1967$.

[2] A. Baker et G. WÜstholz. Logarithmic forms and group varieties. J. reine angew. Math, 442 :19-62, 1993.

[3] W. BAnAszczyK. New bounds in some transference theorems in the geometry of numbers. Math. Ann., 296(4) :625-635, 1993.

[4] D. Bertrand. Transcendental methods in arithmetic geometry. In Analytic Number Theory (Tokyo 1988), volume 1434 de Lecture Notes in Math., 31-44. Springer, 1990.

[5] C. Birkenhake et H. Lange. Complex abelian varieties, volume 302 de Grundlehren Der Mathematischen Wissenschaften. Springer-Verlag Berlin, 1992.

[6] S. Bosch, W. LütKebohmert et M. Raynaud. Néron models, volume 21 de Ergebnisse der Mathematik und ihrer Grenzgebiete (3). Springer-Verlag, 1990.

[7] J.-B. Bost. Semi-stability and heights of cycles. Invent. Math., 118(2) :223-253, 1994.

[8] J.-B. Bost. Arakelov geometry of abelian varieties. In Conference on Arithmetical Geometry, volume 96-51, Max Planck Institut für Mathematik Bonn, mars 1996. Notes manuscrites.

[9] J.-B. Bost. Intrinsic heights of stable varieties and abelian varieties. Duke Math. J., 82(1) :21-70, 1996.

[10] J.-B. Bost. Périodes et isogénies des variétés abéliennes sur les corps de nombres (d'après D. Masser et G. Wüstholz). In Séminaire Bourbaki, volume 237 de Astérisque, 115-161. Société Mathématique de France, 1996.

[11] J.-B. Bost. Cours de DEA à l'Institut Henri Poincaré (Paris), 1997 et 1999. Notes manuscrites.

[12] J.-B. Bost. Algebraic leaves of algebraic foliations over number fields. Publ. Math. Inst. Hautes Études Sci, $93: 161-221,2001$.

[13] J.-B. Bost, H. Gillet et C. SoulÉ. Heights of projective varieties and positive Green forms. J. Amer. Math. Soc., 7(4) :903-1027, 1994. 
[14] S. Brulltet. D'une mesure d'approximation simultanée à une mesure d'irrationalité : le cas de $\Gamma(1 / 4)$ et $\Gamma(1 / 3)$. Acta Arith., 104(3) :243-281, 2002.

[15] M. Chardin. Contributions à l'algèbre commutative effective et à la théorie de l'élimination. Thèse de doctorat, Université Paris VI (Jussieu), Février 1990.

[16] D.V. Chudnovsky et G.V. Chudnovsky. Padé approximations and diophantine geometry. Proc. Natl. Acad. Sci. USA, volume 82, 2212-2216, 1985.

[17] G.V. Chudnovsky. Measures of irrationality, transcendence and algebraic independence. In Recent progress in Number Theory, Journées arithmétiques (Exeter, 1980), volume 56 de London Math. Soc. Lecture Note Ser., 11-82. Cambridge Univ. Press., 1982.

[18] P.L. Cijsouw et M. Waldschmidt. Linear forms and simultaneous approximations. Compos. Math., 34 :173$197,1977$.

[19] J. CoAtes et S. LANG. Diophantine approximation on Abelian varieties with complex multiplication. Invent. Math., 34(2) :129-133, 1976.

[20] S. DAvid. Minorations de formes linéaires de logarithmes elliptiques, volume 62 de Mémoire de la Société Mathématique de France. S. M. F., 1995.

[21] S. David et M. Hindry. Minoration de la hauteur de Néron-Tate sur les variétés abéliennes de type C. M. J. reine angew. Math., $529: 1-74,2000$.

[22] S. David et N. Hirata-Kohno. Linear forms in elliptic logarithms. Prépublication 2005.

[23] S. David et N. Hirata-Kohno. Recent progress on linear forms in elliptic logarithms. In A Panorama in Number Theory. Cambridge Univ. Press, Septembre 2002. Éd. par G. Wüstholz.

[24] S. DAvid et P. Philippon. Minorations des hauteurs normalisées des sous-variétés de variétés abeliennes II. Comment. Math. Helv, 77(4) :639-700, 2002.

[25] N.I. Fel'dman et Yu.V. NeSterenko. Number Theory IV. Transcendental numbers, volume 44 de Encyclopaedia of Mathematical Sciences. Springer-Verlag Berlin, 1998. A.N. Parshin, I.R. Shafarevich (Eds).

[26] É. Gaudron. Mesure d'indépendance linéaire de logarithmes dans un groupe algébrique commutatif. Thèse de doctorat, Université Jean Monnet de Saint-Étienne (France), Décembre 2001 (disponible sur http ://tel.ccsd.cnrs.fr)

[27] É. Gaudron. Mesure d'indépendance linéaire de logarithmes dans un groupe algébrique commutatif. $C$. $R$. Acad. Sci, I 333 :1059-1064, 2001.

[28] É. Gaudron. Mesures d'indépendance linéaire de logarithmes dans un groupe algébrique commutatif. Invent. Math., 162(1) :137-188, 2005.

[29] É. Gaudron. Étude du cas rationnel de la théorie des formes linéaires de logarithmes. Prépublication nº 646 de l'Institut Fourier, Grenoble, 2004.

[30] J. Gebel, A. Ретнӧ et H.G. Zimmer. Computing integral points on elliptic curves. Acta. Arith., 68 :171-192, 1994.

[31] H. Gillet et C. Soulé. An Arithmetic Riemann-Roch Theorem. Invent. Math., 110 :473-543, 1992.

[32] P. Graftienux. Groupes formels et critères d'isogénie. Thèse de doctorat, Université Paris VI, mars 1998.

[33] P. Graftieaux. Formal Groups and Isogeny Theorem. Duke Math. J., 106 :81-121, 2001.

[34] P. Graftieaux. Formal subgroups of abelian varieties. Invent. Math., 145 :1-17, 2001.

[35] N. Hirata-Kohno. Formes linéaires de logarithmes de points algébriques sur les groupes algébriques. Invent. Math., $104: 401-433,1991$.

[36] N. Hirata-Kohno. Approximations simultanées sur les groupes algébriques commutatifs. Compos. Math., $86: 69-96,1993$.

[37] N. Hirata-Kohno. Une relation entre les points entiers sur une courbe algébrique et les points rationnels de la jacobienne. In Advances in number theory (Kingston, ON, 1991), p. 421-433. Oxford Univ. Press, New York, 1993.

[38] T. HondA. On the theory of commutative formal groups. J. Math. Soc. Jap., 22 :213-246, 1970.

[39] J.I. Igusa. Theta Functions, volume 194 de Grundlehren der mathematischen Wissenschaften. Springer-Verlag, 1972.

[40] S. LANG. Diophantine approximations on toruses Amer. J. Math., 86 :521-533, 1964.

[41] S. LANG. Diophantine approximation on abelian varieties with complex multiplications. Advances in Math., $17(3): 281-336,1975$.

[42] S. LAnG. Elliptic curves : Diophantine analysis, volume 231 de Grundlehren der mathematischen Wissenschaften. Springer-Verlag, 1978.

[43] H. LANGE. Families of translations of commutative algebraic groups. J. Algebra, 109 :260-265, 1987.

[44] J. Martinet. Perfect lattices in Euclidean spaces, volume 327 de Grundlehren der mathematischen Wissenschaften. Springer-Verlag, 2003.

[45] D. Masser. Elliptic functions and transcendence, volume 437 de Lecture Notes in Math. Springer-Verlag, 1975. 
[46] D. Masser. Linear forms in algebraic points of abelian functions. I.(II.). Math. Proc. Cambridge Philos. Soc., 77 (79): $499-513$ (55-70), 1975 (1976).

[47] D. Masser. Small values of heights on families of abelian varieties. In Diophantine Approximation and Transcendence Theory, volume 1290 de Lecture Notes in Math. Springer-Verlag, 1987. Éd. par G. Wüstholz.

[48] D. Masser et G. Wüstholz. Estimating isogenies on elliptic curves. Invent. Math., 100(1) :1-24, 1990.

[49] D. Masser et G. Wüstholz. Periods and minimal abelian subvarieties. Ann. of Math., 137(2) :407-458, 1993.

[50] D. Masser et G. Wüstholz. Isogeny estimates for abelian varieties, and finiteness theorems. Ann. of Math., $137(3): 459-472,1993$.

[51] L. Moret-Bailly. Pinceaux de variétés abéliennes, volume 129 de Astérisque. Société Mathématique de France, 1985.

[52] L. Moret-Bailly. Sur l'équation fonctionnelle de la fonction thêta de Riemann. Compositio Math., 75(2) :203217,1990

[53] D. Mumford. Abelian varieties, Tata Institute of Fundamental Research Studies in Mathematics, No. 5. Published for the Tata Institute of Fundamental Research, Bombay, 1970.

[54] Yu. V. Nesterenko et P. Philippon, éditeurs. Introduction to Algebraic Independence, volume 1752 de Lecture Notes in Math. Springer-Verlag, 2001.

[55] F. Pellarin. Sur la distance d'un point algébrique à l'origine dans les variétés abéliennes. J. Number Theory, $88: 241-262,2001$.

[56] P. Philippon. Nouveaux lemmes de zéros dans les groupes algébriques commutatifs. Rocky Mt. J. Math., 26(3) :1069-1088, 1996.

[57] P. Philippon et M. Waldschmidt. Formes linéaires de logarithmes sur les groupes algébriques commutatifs. Illinois J. Math., 32(2) :281-314, 1988.

[58] P. Philippon et M. Waldschmidt. Formes linéaires de logarithmes simultanées sur les groupes algébriques commutatifs. In Séminaire de Théorie des Nombres Paris 1986 - 87, volume 75 de Progress in Mathematics, 313-347. Birkhäuser Boston, Inc., 1989. Éd. par C. Goldstein.

[59] H. Randriambololona. Hauteurs de sous-schémas, géométrie d'Arakelov des schémas de Hilbert et exemples d'utilisation de méthodes arakeloviennes en théorie de l'approximation diophantienne. Thèse de doctorat, Université Paris XI, Janvier 2002.

[60] G. RÉmond. Élimination multihomogène. Chapitre 5 de 54 .

[61] M. Raynaud. Hauteurs et isogénies. In «Séminaire sur les pinceaux arithmétiques : La conjecture de Mordell », Exposé VII, 1985, Astérisque 127, Société Mathématique de France. Éd. par L. Szpiro.

[62] G. RÉmond et F. URfels. Approximation diophantienne de logarithmes elliptiques p-adiques. J. Number Theory, 57(1):133-169, 1996.

[63] W.M. Schmidt. On heights of algebraic subspaces and diophantine approximations. Ann. of Math., 85:430-472, 1967.

[64] J.H. Silverman. Heights and Elliptic curves. Chapitre X de Arithmetic Geometry, 1986, Springer-Verlag. Éd. par G. Cornell et J.H. Silverman.

[65] N.P. Smart. The Algorithmic Resolution of Diophantine Equations, volume 41 de Student Texts, London Mathematical Society. Cambridge University Press, 1998.

[66] R.J. Stroeker et N. Tzanakis. Solving elliptic diophantine equations by estimating linear forms in elliptic logarithms. Acta. Arith., 67 :177-196, 1994.

[67] M. Waldschmidt. Approximation diophantienne dans les groupes algébriques commutatifs. (I) : Une version effective du théorème du sous-groupe algébrique. J. reine angew. Math, 493 :61-113, 1997.

[68] M. Waldschmidt. Diophantine Approximation On Linear Algebraic Groups, volume 326 de Grundlehren der mathematischen Wissenschaften. Springer-Verlag, 2000.

[69] G. Wüstholz. Some remarks on a conjecture of Waldschmidt. In Approximations diophantiennes et nombres transcendants (Luminy 1982), volume 31 de Prog. Math., 329-336, 1983.

[70] G. Wüstholz. Algebraische punkte auf analytischen untergruppen algebraischer gruppen. Ann. of Math., $129(3): 501-517,1989$.

Université Grenoble I, Institut Fourier.

UMR 5582, BP 74

38402 Saint-Martin-d'Hères Cedex, France.

Courriel : Eric.Gaudron@ujf-grenoble.fr

Page internet : http ://www-fourier.ujf-grenoble.fr/ gaudron 